

\section{INFORMATION TO USERS}

This reproduction was made from a copy of a manuscript sent to us for publication and microfilming. While the most advanced technolugy has been used to photograph and reproduce this manuscript. the quality of the reproduction is heavily dependent upon the quality of the material submitted. Pages in any manuscript may have indistinct print. In all cases the best available copy has been filmed.

The following explanation of techniques is provided to help clarify notations which may appear on this reproduction.

1. Manuscripts may not always be complete. When it is not possible to obtain missing pages, a note appears to indicate this.

2. When copyrighted materials are removed from the manuscript, a note appears to indicate this.

3. Oversize materials (maps, drawings. and charts) are photographed by sectioning the original, beginning at the upper left hand corner and continuing from left to right in equal sections with small overlaps. Each oversize page is also filmed as one exposure and is avallable, for an additional charge. as a standard $35 \mathrm{~mm}$ slide or in black and white paper format."

4. Most photographs reproduce acceptably on positive microfilm or microfiche but lack clarity on xerographic coples made from the microfilm. For an additional charge, all photographs are avallable in black and white standard $35 \mathrm{~mm}$ slide format.*

*For more information about black and white slides or enlarged paper reproductions, please contact the Dissertations Customer Services Department.

\section{U.1.I.I $\begin{aligned} & \text { Dissertation } \\ & \text { Information Service }\end{aligned}$}

University Microfilms International A Bell \& Howell Information Company 300 N. Zeeb Road, Ann Arbor, Michigan 48106 
Fok, Fredric Yat Sing

K.PULSE ESTIMATION FROM THE IMPULSE RESPONSE OF A TARGET

The Ohio State University

PH.D. 1986

University

Microfilms

International $300 \mathrm{~N}$. Zeeb Road, Ann Arbor, M1 48106 


\title{
K-PULSE ESTIMATION FROM THE IMPULSE RESPONSE OF A TARGET
}

\section{DISSERTATION}

\begin{abstract}
Presented in Partial Fulfillment of the Requirements for the Degree Doctor of Philosophy in the Graduate

School of The Ohio State University
\end{abstract}

By

Fredric Yat Sing Fok, B. A. Sc., M. Sc.

The Ohio State University

1986

Dissertation Committee:

Approved by

David L. Moffatt

Leon Peters, Jr.

Walter D. Burnside

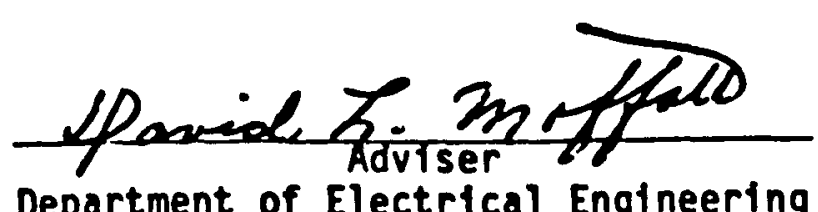


To late Professor Kennaugh, Whose ingenuity I envy. To my Parents, Whom I endear. 


\section{ACKNOWLEDGMENT}

I would like to thank my advisor Professor David L. Moffatt for his guidance in the preparation of this dissertation. I would also like to thank Dr. Nan Wang for his ti.ne and comments in the numerous discussions to make this dissertation possible. I wish to express my thanks to Professor Leon Peters for being my M. S. advisor and reviewing this dissertation carefully. I also want to thank Professor Walter D. Burnside for his review and constructive criticisms. Many thanks go to all my friends at the Electroscience Laboratory for their informative discussions. I thank Mr. J. M. Gibson for his drafting. Last but not least, I wish to thank Ms. Becky Thornton for her patience in typing and putting the finishing touches on this dissertation. 


\section{VITA}

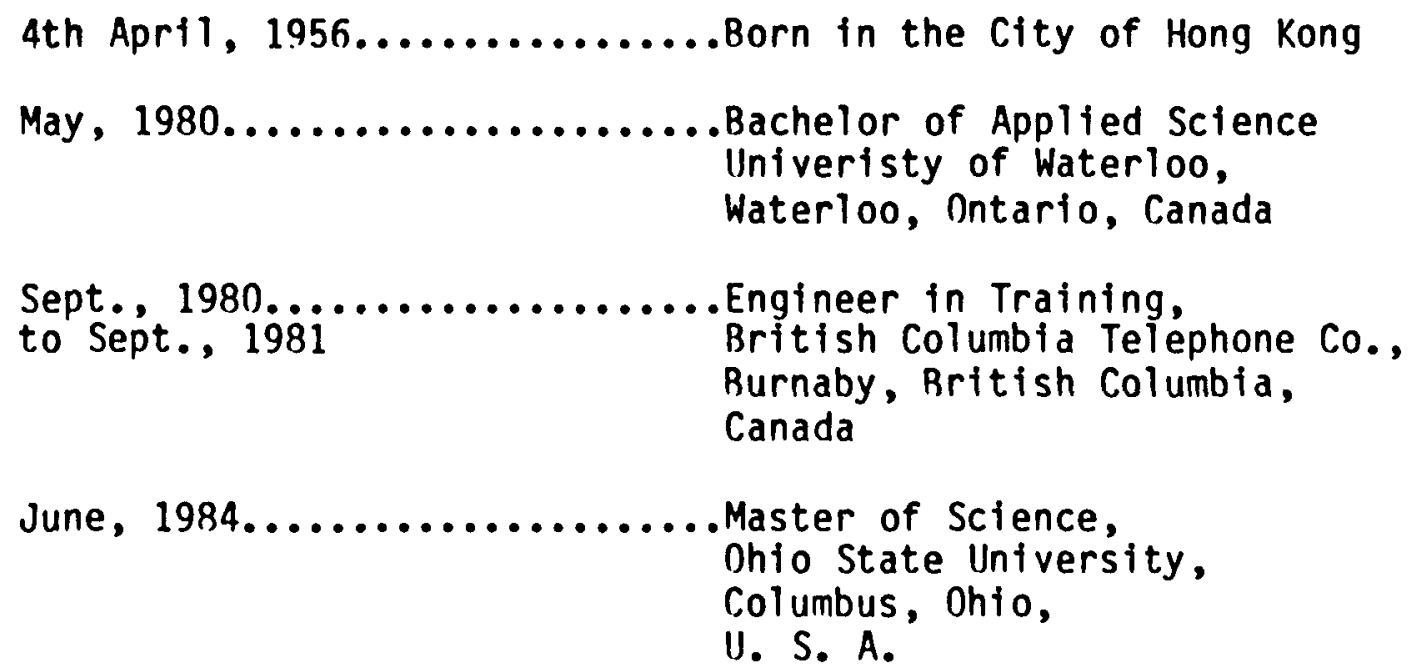

PUBLICATIONS

Thesis: Space-frequency Sampling Criteria For Electromagnetic Scattering of a Finite nbject

FIELDS OF STUDY

Major Field: Electrical Engineering

Studies in Electromagnetic Theory: Professor n. L. Moffatt Professor R. G. Kouyoumjian

Studies in Communication Theory: Professor R. T. Compton, Jr.

Studies in Control Theory: Professor R. E. Fenton

Studies in Mathematics: Professor S. Drobot 


\section{TABLE OF CONTENTS}

DEDICATION

ACKNOWLEDGEMENT

VITA

LIST OF TABLES

vi

LIST OF FIGURES

viit

CHAPTER

PAGE

I INTRODUCTION

II THEORY

A. K-PULSE DERIVATION

B. POLE EXTRACTION

III APPLICATION TO THEORETICAL DATA

A. EXAMPLE I

B. EXAMPLE II

C. EXAMPLE III

D. EXAMPLE IV

E. EXAMPLE V

F. EXAMPLE VI

G. EXAMPLE VII

IV ESTIMATION OF THE DURATION OF THE K-PULSE

84

$\checkmark$ APPLICATION TO NOISY AND MEASURED DATA

131

VI CONCLUSIONS AND RECOMMENDATIONS

APPENDICES

$\begin{array}{lll}\text { A PROCEDURES TO ESTIMATE THE K-PULSE } & 171\end{array}$

B A TARGET DISCRIMINATION SCHEME 182

$\begin{array}{lrr}\text { C } & \text { K-PULSE AND E-PULSE } & 188\end{array}$

D PROOF THAT A MEMORPHIC FUNCTION CAN BE FACTORED INTO A RATIO OF TWO ENTIRE FIJNCTIONS 


\section{LIST OF TABLES}

Table

Page

3.1. A Comparison of the First Ten Exact Poles and Estimated Zeros in the s-domain (Upper Left Half Plane) for the Simulated Model in Equation (3.2)

3.2. A Comparison of the First Six Exact Poles and Estimated Zeros in the $s$-domain (Upper Left Half Plane) for the Conducting Thin Straight Wire

3.3. A Comparison of the First Ten Exact Poles and Estimated Zeros in the s-domain (Upper Left Half Plane) for the Conducting Thin Circular Loop

3.4. A Comparison of the First Ten Exact Poles and Estimated Zeros in the s-domain (Upper Left Half Plane) for the Conducting Sphere

3.5. A Comparison of the Estimated First Ten Poles in the $s$-domain (Upper Left Half Plane) for the Conducting Circular Disk

4.1. Radian Frequency Difference Between Consecutive Poles

4.2. Summary of Radian Frequency Differences and Its Corresponding Time

4.3. A Comparison of the Zeros Obtained Using Two $K$-pulses with Different Durations (The Target is a Straight Wire)

4.4. A Comparison of the Zeros Obtained Using Two $K$-pulses with Different Durations (The Target is a Circular Loop)

4.5. A Comparison of the Zeros Obtained lising Two K-pulses with Different Durations (The Simulated Model is in Equation (3.2))

4.6. A Comparison of the Zeros Obtained Using Two K-pulses with Different Durations (The Target is a Conducting Sphere)

4.7. A Comparison of the Zeros Obtained Using Two K-pulses with Different Durations (The Target is a Circular Disk) 
5.1. A Comparison of the First Six Exact Poles and Estimated Zeros in the $s$-domain (Upper Left Half Plane) for the Thin Circular Loop

5.2. The First Five Estimated Poles in the s-domain (Upper Left Half Plane) for the Finite Circular Waveguide

A.1. A Comparison of the First Six Exact Poles and Estimated Zeros in the s-domain (Upper Left Half Plane) for the Conducting Thin Circular Loop 
2.1. An illustration of linear interpolation and extrapolation $\left(a_{0}\right)$ of samples and their derivatives.

3.1. The simple target models used to illustrate the method of K-pulse estimation. The orientation geometry is also given.

3.2. Response of the grounded dielectric slab to a normally incident plane wave with impulsive time dependence. (each diamond's height represents the weighting of an impulse)

3.3. Response of the grounded dielectric slab to a set of unit impulses (26) which represents the first guess for the $K$-pulse. (each diamond's height represents the weighting of an impulse).

3.4. The K-pulse for normal incidence on the grounded dielectric slab resulted after optimization with the initial guess corresponding to Figure 3.3. (each diamond's height represents the weighting of an impulse).

3.5. Response of the grounded dielectric slab to the K-pulse shown in Figure 3.4. (each diamond's height represents the weighting of an impulse).

3.6. Two other initial guesses for the K-pulse which after optimization yield the same K-pulse as Figure 3.4 .

3.7. Impulse response (Fourier synthesized) of the grounded dielectric slab to a normaliy incident plane wave. $(K L=0.01 \pi(0.01 \pi) 100 \pi)$.

3.8. Response of the grounded dielectric slab to a set of unit impulses (11) which represents the first guess for the K-pulse.

3.9. The $K$-pulse for normal incidence on the grounded dielectric slab resulted after optimization with the initial guess corresponding to Figure 3.8. (each diamond's height represents the weight of an impulse).

3.10. Response of the grounded dielectric slab to the normal incident of the K-pulse shown in Figure 3.9. 
3.11. The exact and approximate $K$-pulse for the simulated model given in Equation (3.2).

3.12. Impulse response and response to the approximate $K$-pulse shown in Figure 3.11 for the simulated model in Equation (3.2). Both responses have an impulsive singularity at the start of the waveform. $(K L=0.2 \pi(0.2 \pi) 400 \pi)$.

3.13. K-pulse obtained using the Fourier synthesized impulse response of the conducting straight wire at $\phi=30^{\circ}$ with $K L=0.08 \pi(0.08 \pi) 8 \pi$.

3.14. Impulse response and response to the $K$-pulse shown in Figure 3.13 for the straight wire at $\phi=30^{\circ}$ with $K L=0.08 \pi(0.08 \pi) 8 \pi$.

3.15. Impulse response and response to the $K$-pulse shown in Figure 3.13 for the straight wire at $i=60^{\circ}$ with $k L=0.08 \pi(0.08 \pi) 8 \pi$.

3.16. Impulse response and response to the K-pulse shown in Figure 3.13 for the straight wire at $\phi=90^{\circ}$ with $K L=0.08 \pi(0.08 \pi) 8 \pi$.

3.17. K-pulse obtained using the Fourier synthesized impulse response of the conducting circular loop at $\theta=45^{\circ}$, $\hat{\theta}$-polarization with $k r=0.02 \pi(0.02 \pi) 4 \pi$.

3.18. Impulse response and response to the $K$-pulse shown in Figure 3.17 for the conducting circular loop at $\theta=45^{\circ}$, $\theta$-polarization with $k r=0.02 \pi(0.02 \pi) 4 \pi$.

3.19. Impulse response and response to the K-pulse shown in Figure 3.17 for the conducting circular loop at $\theta=0^{\circ}$, $\phi$-polarization with $\mathrm{kr}=0.02 \pi(0.02 \pi) 4 \pi$.

3.20. Impulse response and response to the $K$-pulse shown in Figure 3.17 for the conducting circular loop at $\theta=45^{\circ}$, $\phi$-polarization with $\mathrm{kr}=0.02 \pi(0.02 \pi) 4 \pi$.

3.21. Impulse response and response to the $K$-pulse shown in Figure 3.17 for the conducting circular loop at $\theta=90^{\circ}$, $\phi$-polarization with $k r=0.02 \pi(0.02 \pi) 4 \pi$. 
3.22. K-pulse obtained using the Fourier synthesized impulse response of the conducting sphere in the backscattered direction with $\mathrm{kr}=0.1(0.1) 66$.

3.23. Impulse response and response to the K-pulse shown in Figure 3.22 for the conducting sphere in the backscattered direction with $\mathrm{kr}=0.1(0.1) 66$.

3.24. Impulse response and response to the K-pulse shown in Figure 3.22 for the conducting sphere in the backscattered direction with $\mathrm{kr}=0.1(0.1) 33.5$.

3.25. Impulse response and response to the $K$-pulse shown in Figure 3.22 for the conducting sphere at a bistatic angle of $\phi=90^{\circ}$, vertical polarization with $k r=0.1(0.1) 33.5$.

3.26. Impulse response and response to the K-pulse shown in Figure 3.22 for the conducting sphere at a bistatic angle of $\phi=90^{\circ}$, horizontal polarization with $k r=0.1(0.1) 33.5$.

3.27. Impulse response and response to the $K$-pulse shown in Figure 3.22 for the conducting sphere at a bistatic angle of $\phi=180^{\circ}$, vertical polarization with $k r=0.1(0.1) 33.5$.

3.28. K-pulse obtained using the Fourier synthesized impulse response of the conducting circular disk at $\theta=45^{\circ}$, $\hat{\theta}$-polarization with $\mathrm{kr}=0.16(0.16) 16$.

3.29. Impulse response and response to the $K$-pulse shown in Figure 3.28 for the conducting circular disk at $\theta=45^{\circ}$, $\hat{\theta}$-polarization with $\mathrm{kr}=0.16(0.16) 16$.

3.30. Impulse response and response to the $K$-pulse shown in Figure 3.28 for the conducting circular disk at $\theta=0^{\circ}$, $\hat{\theta}$-polarization with $\mathrm{kr}=0.16(0.16) 16$.

3.31. Impulse response and response to the K-pulse shown in Figure 3.28 for the conducting circular disk at $\theta=45^{\circ}$, $\hat{\phi}$-polarization with $\mathrm{kr}=0.16(0.16) 16$. 
3.32. Impulse response and response to the $K$-pulse shown in Figure 3.28 for the conducting circular disk at $\theta=90^{\circ}$, $\hat{\phi}$-polarization with $\mathrm{kr}=0.16(0.16) 16$.

3.33. K-pulse obtained using the Fourier synthesized impulse response of the conducting circular disk at $\theta=45^{\circ}$, $\hat{\phi}$-polarization with $\mathrm{kr}=0.16(0.16) 16$.

3.34. Impulse response and response to the $K$-pulse shown in Figure 3.33 for the conducting circular disk at $\theta=45^{\circ}$, ф-polarization with $\mathrm{kr}=0.16(0.16) 16$.

3.35. Impulse response and response to the $K$-pulse shown in Figure 3.33 for the conducting circular disk at $\theta=0^{\circ}$, $\hat{\theta}$-polarization with $\mathrm{kr}=0.16(0.16) 16$.

3.36. Impulse response and response to the $K$-pulse shown in Figure 3.33 for the conducting circular disk at $\theta=45^{\circ}$, $\hat{\theta}$-polarization with $\mathrm{kr}=0.16(0.16) 16$.

3.37. Impulse response and response to the $K$-pulse shown in Figure 3.33 for the conducting circular disk at $\theta=90^{\circ}$, $\hat{\phi}$-polarization with $\mathrm{kr}=0.16(0.16) 16$.

4.1. K-pulse obtained using the Fourier synthesized impulse response of the conducting straight wire at $\phi=30^{\circ}$ with $k L=0.08 \pi(0.08 \pi) 8 \pi$. The K-pulse duration is $3 L / C$.

4.2. Impulse response and response to the $K$-pulse shown in Figure 4.1 for the straight wire at $\phi=30^{\circ}$ with $k L=0.08 \pi(0.08 \pi) 8 \pi$.

4.3. K-pulse obtained using the Fourier synthesized impulse response of the conducting straight wire at $\phi=30^{\circ}$ with $k L=0.08 \pi(0.08 \pi) 8 \pi$. The K-pulse duration is $4 L / C$.

4.4. Impulse response and response to the $K$-pulse shown in Figure 4.3 for the straight wire at $\phi=30^{\circ}$ with $K L=0.08 \pi(0.08 \pi) 8 \pi$. 
4.5. Impulse response and response to the "K-pulse" shown in Figure 4.6 for the straight wire at $\phi=30^{\circ}$ with $K L=0.08 \pi(0.08 \pi) 8 \pi$.

4.6. "K-pulse" obtained using the Fourier synthesized impulse response of the conducting straight wire at $\phi=30^{\circ}$ with $K L=0.08 \pi(0.08 \pi) 8 \pi$. The "K-pulse" duration is $L / C$.

4.7. K-pulse obtained using the Fourier synthesized impulse response of the conducting circular loop at $\theta=45^{\circ}$ and $\hat{\theta}$-polarization with $k r=0.02 \pi(0.02 \pi) 4 \pi$. The $K$-pulse duration is $3 \pi r / c$.

4.8. Impulse response and response to the $k$-pulse shown in

Figure 4.7 for the circular loop at $\theta=45^{\circ}$ and

$\hat{\theta}$-polarization with $\mathrm{kr}=0.02 \pi(0.02 \pi) 4 \pi$.

4.9. K-pulse obtained using the Fourier synthesized impulse response of the conducting circular loop at $\theta=45^{\circ}$ and $\hat{\theta}$-polarization with $k r=0.02 \pi(0.02 \pi) 4 \pi$. The $k$-pulse duration is $4 \pi r / c$.

4.10. Impulse response and response to the $K$-pulse shown in

Figure 4.9 for the circular loop at $\theta=45^{\circ}$ and

$\hat{\theta}$-polarization with $\mathrm{kr}=0.02 \pi(0.02 \pi) 4 \pi$.

4.11. Impulse response and response to the "K-pulse" shown in Figure 4.12 for the circular loop at $\theta=45^{\circ}$ and $\hat{\theta}$-polarization with $\mathrm{kr}=0.02 \pi(0.02 \pi) 4 \pi$.

4.12. "K-pulse" obtained using the Fourier synthesized impulse response of the conducting circular loop at $\theta=45^{\circ}$ and $\hat{\theta}$-polarization with $\mathrm{kr}=0.02 \pi(0.02 \pi) 4 \pi$. The "K-pulse" duration is $\pi r / c$.

4.13. K-pulse obtained using the Fourier synthesized impulse response of the simulated model given in Equation (3.2). The K-pulse duration is $1.5 \mathrm{~L} / \mathrm{C}$.

4.14. Impulse response and response to the $K$-pulse shown in Figure 4.13 for the simulated model given in Equation (3.2). $\quad(k L=0.05 \pi(0.05 \pi) 100 \pi)$. 
4.15. K-pulse obtained using the Fourier synthesized impulse response of the simulated model given in Equation (3.2). The $K$-pulse duration is $2 L / C$.

4.16. Impulse response and response to the $K$-pulse shown in Figure 4.15 for the simulated model given in Equation (3.2). $(k L=0.05 \pi(0.05 \pi) 100 \pi)$.

4.17. "K-pulse" obtained using the Fourier synthesized impulse response of the simulated model given in Equation (3.2). The "K-pulse" duration is $0.5 \mathrm{~L} / \mathrm{C}$.

4.18. Impulse response and response to the "K-pulse" shown in Figure 4.17 for the simulated model given in Equation (3.2). $(k L=0.05 \pi(0.05 \pi) 100 \pi)$.

4.19. K-pulse obtained using the Fourier synthesized impulse response of the conducting sphere in the the backscattered direction with $k r=0.1(0.1) 33.5$. The K-pulse duration is $3 \pi r / c$.

4.20. Impulse response and response to the $K$-pulse shown in Figure 4.19 for the conducting sphere in the backscattered direction with $k r=0.1(0.1) 33.5$. The amplitude scale is expanded by 100 .

4.21. K-pulse obtained using the Fourier synthesized impulse response of the conducting sphere in the backscattered direction with $k r=0.1(0.1) 33.5$. The $K$-pulse duration is $4 \pi r / c$.

4.22. Impulse response and response to the $K$-pulse shown in Figure 4.21 for the conducting sphere in the backscattered direction with $k r=0.1(0.1) 33.5$. The amplitude scale is expanded by 100 .

4.23. "K-pulse" obtained using the Fourier synthesized impulse response of the conducting sphere in the backscattered direction with $k r=0.1(0.1) 33.5$. The "k-pulse" duration is $\pi r / c$.

4.24. Impulse response and response to the "K-pulse" shown in Figure 4.23 for the conducting sphere in the backscattered direction with $\mathrm{kr}=0.1(0.1) 33.5$. The amplitude scale is expanded by 100 . 
Figure

4.25. K-pulse obtained using the Fourier synthesized impulse response of the conducting circular disk at $\theta=45^{\circ}$, $\hat{\theta}$-polarization with $k r=0.16(0.16) 16$. The $K$-pulse duration is $3 \pi r / c$.

4.26. Impulse response and response to the $K$-pulse shown in Figure 4.25 for the conducting circular disk at $\theta=45^{\circ}$, $\hat{\theta}$-polarization with $\mathrm{kr}=0.16(0.16) 16$. The amplitude scale is expanded by 100 .

4.27. K-pulse obtained using the Fourier synthesized impulse response of the conducting circular disk at $\theta=45^{\circ}$, $\hat{\theta}$-polarization with $\mathrm{kr}=0.16(0.16) 16$. The $\mathrm{K}$-pulse duration is $4 \pi r / c$.

4.28. Impulse response and response to the $K$-pulse shown in Figure 4.27 for the conducting circular disk at $\theta=45^{\circ}$, $\hat{\theta}$-polarization with $k r=0.16(0.16) 16$. The amplitude scale is expanded by 100 .

4.29. "K-pulse" obtained using the Fourier synthesized impulse response of the conducting circular disk at $\theta=45^{\circ}$, $\hat{\theta}$-polarization with $\mathrm{kr}=0.16(0.16) 16$. The "K-pulse" duration is $\pi r / c$.

4.30. Impulse response and response to the "K-pulse" shown in Figure 4.29 for the conducting circular disk at $\theta=45^{\circ}$, $\hat{\theta}$-polarization with $\mathrm{kr}=0.16(0.16) 16$. The amplitude scale is expanded by 100 .

5.1. An fllustration of the geometry of a finite circular waveguide.

5.2. Fourier synthesized impulse response of the circular loop at $\theta=45^{\circ}, \theta$-polarization with Gaussian noise $N(0,0.01 \mathrm{~V})$. $(k r=0.02 \pi(0.02 \pi) 4 \pi)$. 
5.3. K-pulse obtained using the impulse response shown in. Figure 5.2.

5.4. Impulse response and response to the $k$-pulse shown in Figure 5.3 for the circular loop at $\theta=45^{\circ}, \hat{\theta}$-polarization with Gaussian noise $N(0,0.01 V)$. $(k r=0.02 \pi(0.02 \pi) 4 \pi)$.

5.5. Impulse response and response to the $K$-pulse shown in Figure 5.3 for the circular loop at $\theta=45^{\circ}, \hat{\theta}$-polarization with no noise. $(k r=0.02 \pi(0.02 \pi) 4 \pi)$.

5.6. Impulse response and response to the K-pulse shown in Figure 5.3 for the circular loop at $\theta=0^{\circ}, \hat{\phi}$-polarization with no noise. $(k r=0.02 \pi(0.02 \pi) 4 \pi)$.

5.7. Impulse response and response to the K-pulse shown in Figure 5.3 for the circular loop at $\theta=45^{\circ}, \hat{\phi}$-polarization with no noise. $(k r=0.02 \pi(0.02 \pi) 4 \pi)$.

5.8. Impulse response and response to the $K$-pulse shown in Figure 5.3 for the circular loop at $\theta=90^{\circ}, \ddot{\phi}$-polarization with no noise. $(k r=0.02 \pi(0.02 \pi) 4 \pi)$.

5.9. Fourier synthesized impulse response of the circular loop at $\theta=45^{\circ}, \hat{\theta}$-polarization with Gaussian noise $N(0,0.05 \mathrm{~V})$. $(k r=0.02 \pi(0.02 \pi) 4 \pi)$.

5.10. K-pulse obtained using the impulse response shown in Figure 5.9.

5.11. Impulse response and response to the $K$-pulse shown in Figure 5.10 for the circular loop at $\theta=45^{\circ}, \hat{\theta}$-polarization with Gaussian noise $N(0,0.05 V)$. ( $k r=0.02 \pi(0.02 \pi) 4 \pi)$.

5.12. Impulse response and response to the $K$-pulse shown in Figure 5.10 for the circular loop at $\theta=45^{\circ}, \hat{\theta}$-polarization with no noise. $(k r=0.02 \pi(0.02 \pi) 4 \pi)$. 
5.13. Impulse response and response to the $K$-pulse shown in .

Figure 5.10 for the circular loop at $\theta=0^{\circ}, \hat{\phi}$-polarization with no noise. $(k r=0.02 \pi(0.02 \pi) 4 \pi)$.

5.14. Impulse response and response to the $K$-pulse shown in Figure 5.10 for the circular loop at $\theta=45^{\circ}, \hat{\phi}$-polarization with no noise. $(k r=0.02 \pi(0.02 \pi) 4 \pi)$.

5.15. Impulse response and response to the $K$-pulse shown in Figure 5.10 for the circular loop at $\theta=90^{\circ}, \hat{\phi}$-polarization with no noise. $(k r=0.02 \pi(0.02 \pi) 4 \pi)$.

5.16. Fourier synthesized impulse response of the open ended finite circular waveguide at $\theta=0^{\circ}, \phi=0^{\circ}$, and vertical polarization with $\mathrm{kr}=0.00665(0.00665) 12$.

5.17. Same waveform as in Figure 5.16, except the time scale is given up to $7 \pi r / c$ - equivalent to the Fourier synthesized impulse response of a semi-infinite circular waveguide at $\phi=0^{\circ}, \theta=0^{\circ}$, and vertical polarization with $k r=0.00665(0.00665) 12$.

5.:3. K-pulse obtained using the impulse response shown in Figure 5.17.

5.19. Figure 5.17 and response to the K-pulse shown in

Figure 5.18 for the waveform shown in Figure 5.17.

5.20. Fourier synthesized impulse response of the finite circular waveguide with one open end at $\phi=0^{\circ}$, and one closed end at $\phi=180^{\circ}$. The data are measured at $\phi=0^{\circ}, \theta=0^{\circ}$ and vertical polarization with $k r=0.00665(0.00665) 12$.

5.21. Figure 5.20 and response to the K-pulse shown in Figure 5.18 for the waveform shown in Figure 5.20.

5.22. Impulse response and response to the $K$-pulse shown in Figure 5.18 for the open ended finite circular waveguide at $\phi=0^{\circ}, \theta=0^{\circ}$ and vertical polarization with $k r=0.00665(0.00665) 12$.

5.23. K-pulse obtained using the Fourier synthesized impulse response of the open ended finite circular waveguide at $\phi=0^{\circ}, \theta=0^{\circ}$ and vertical polarization with $k r=0.00665(0.00665) 12$. 
5.24. Impulse response and response to the $K$-pulse shown in Figure 5.23 for the open ended finite circular waveguide at $\phi=0^{\circ}, \theta=0^{\circ}$ and vertical polarization with $k r=0.00665(0.00665) 12$.

5.25. Impulse response and response to the $K$-pulse shown in Figure 5.23 for the finite circular waveguide with one open end at $\phi=0^{\circ}$, and one closed end at $\phi=180^{\circ}$. The data are taken at $\phi=0^{\circ}, \theta=0^{\circ}$ and vertical polarization with $\mathrm{kr}=0.00665(0.00665) 12$.

A.1. Fourier synthesized impulse response of the conducting circular loop at $\theta=45^{\circ}, \hat{\theta}$-polarization. $(k r=0.02 \pi(0.02 \pi) 4 \pi)$.

A.2. K-pulse obtained using the Fourier synthesized impulse response of the conducting circular loop at $\theta=45^{\circ}$, $\theta$-polarization. $(k r=0.02 \pi(0.02 \pi) 4 \pi)$ The number of samples used to represent the continuous portion of the $K$-pulse is 50 .

A.3. Impulse response and response to the $K$-pulse shown in Figure A.2 for the conducting circular loop at $\theta=45^{\circ}$, $\hat{\theta}$-polarization. $(k r=0.02 \pi(0.02 \pi) 4 \pi)$.

A.4. K-pulse obtained using the Fourier synthesized impulse response of the conducting circular loop at $\theta=45^{\circ}$, $\hat{\phi}$-polarization. $(k r=0.02 \pi(0.02 \pi) 4 \pi)$. The number of samples used to represent the continuous portion of the $K$-pulse is 50 .

A.5. Impulse response and response to the K-pulse shown in Figure A.5 for the conducting circular loop at $\theta=45^{\circ}$, ф-polarization. $(k r=0.02 \pi(0.02 \pi) 4 \pi)$.

B.1. Impulse response and response to the $K$-pulse shown in Figure 3.17 for the thicker conducting circular loop at $\theta=30^{\circ}, \hat{\theta}$-polarization with $\mathrm{kr}=0.042(0.042) 4.2$. 
Figure

B.2. Impulse response and response to the $K$-pulse shown in

Figure 3.17 for the thicker conducting circular loop at $\theta=60^{\circ}, \hat{\theta}$-polarization with $k r=0.042(0.042) 4.2$.

3.3. Block diagram of a target discrimination scheme. 


\section{CHAPTER I}

\section{INTRONUCTION}

The use of electromagnetic scattering information to identify or classify targets has been studied by numerous researchers [1]. The K-pulse concept [2] can be applfed to this problem. Target imaging is another possiblity for the application of the K-pulse. As discussed in [2], one may model or simulate target responses at $N$ aspects and polarizations by a linear distributed parameter network with $\mathrm{N}$ acessible ports. The normalized complex echo signal spectrum $E_{m}(s)$ at port $m$ can be factored into a fraction of two entire functions,

$$
E_{m}(s)=\frac{F_{m}(s)}{K(s)},
$$

if the network contains lumped elements connected by finite delays. The argument that $E_{m}(s)$ is a fraction of two entire functions is further supported by the work of Marin [3]. Marin showed that the operator inverse to the integral operator of the magnetic-field formulation is a memorphic function in the entire complex plane. Since a memorphic function can be factored into a fraction of two entire functions [see Appendix $D], F_{m}(s)$ and $K(s)$ are entire functions of exponential type. Thus, they are time-limited [4]. The inverse Laplace transform of $K(s)$ into the time domain is $k(t)$ - the K-pulse [2]. Roth $E_{m}(s)$ and $F_{m}(s)$ are aspect and polarization dependent. But $K(s)$ is aspect and polarization independent. 
The K-pulse concept is similar to pole-zero cancellation in system control theory. However, the applications in the control theory deal mainly with a finite number of poles and zeros. The poles are only moved to more suitable locations in the complex frequency plane. In this dissertation, zero-insertion by the K-pulse to completely cancel the resonance contributed by all the poles of the target is the ideal concept. In other words, the "system" response due to the K-pulse excitation exhibits no resonance. After $K(s)$ is multiplied onto Equation (1.1), the system response is $F_{m}(s)$ which is an entire function of exponential type; i.e., a time-limited function.

Concepts similar to the K-pulse were employed in communication theory long before Kennaugh and Richmond [5] presented their paper in Washington D.C. in 1972. Gerst and Diamond [4] shaped the input waveform to eliminate the intersymbol interference. Later, Campbell et al. $[6,7]$ extended the theory. The application of the theory in electromagnetic scattering was formalized by Kennaugh [8] in 1975. The concept was not presented to the public until Kennaugh's paper [2] in 1981. Recent7y, Kennaugh, Moffatt and Wang [9] had demonstrated the K-pulse for non-uniform transmission lines. Kim, Wang and Moffatt [10] used some results of Gerst and Diamond [4] to obtain the K-pulse of a circular loop. Rothwell et al. [11] discussed a target discrimination scheme using the Extinction-pulse (E-pulse) which was based on the natural frequencies of the target considered. A disscusion of the relationship between the $K$-pulse and the E-pulse can be found in the Appendix C. 
Although Kennaugh had suggested several techniques to estimate the K-pulse, one technique was never rully applicable to all the targets tested. This dissertation presents an approach to obtain the approximate $K$-pulse of a target from scattering information, without requiring a priori complex natural resonance (pole) information. Specifically, the derivation is based on the impulse response $[12,13]$ of the target. For complicated target geometries, only measured information is available. Thus, impulse and frequency responses of the targets are the commonly available information to work with. Using a $K$-pulse model, the $K$-pulse response is obtained by convolving with the impulse response. The energy outside the $K$-pulse response is minimized using some optimization schemes with respect to the unknowns in the assumed K-pulse model, until the energy content is considered minimum. The dominant poles of the target can also he extracted from the approximate $K$-pulse. A method to obtain these dominant poles is also presented.

In this dissertation, the approaches to obtain the approximate K-pulse and the dominant poles are discussed in Chapter II. Then, both exact and Fourier synthesized impulse responses are used as examples in Chapter III to verify the methods developed in Chapter II. Chapter IV discusses a way to obtain the duration of the K-pulse without knowing the circumnavigational path on the target, which is used to deduce the K-pulse duration for simple geometries. Chapter IV is necessary as it is very difficult to find the circumnavigational path $i_{i}$ i complicated geometrical shape. The targets used for verification in Chapter III and 
IV include a grounded dielectric slab, a low $Q$ target model, a straight wire, a circular loop, a sphere, and a disk. The last four targets are perfectly conducting structures. After verification, the methods are tested with quasi noisy data and experimental data. These results are presented in Chapter $V$. The whole dissertation is then concluded with Chapter VI, together with some recommendations for future studies. Four Appendices are added to clarify a few issues. Appendix A describes the procedures and indicators in using the $K$-pulse estimation method discussed in this dissertation. The circular loop is used as the illustrating example. Appendix $B$ discusses how the $K$-pulse can be used for target discrimination. As mentioned before, a discussion of the K-pulse and the E-pulse waveforms is presented in Appendix C. Appendix $D$ is the proof that a memorphic function in the entire complex plane can be factored into a fraction of two entire functions.

In this dissertation, functions with capital letters are reserved to represent the Laplace transform of the corresponding lower case time functions. Most of the impulse responses are plotted together with the K-pulse responses. This is done so that the two responses can be compared simultaneously. The impulse responses are given as dash lines and the K-pulse responses as solid lines when both responses appear on the same plot. Also, all the initial guesses for the unknowns are unity, unless otherwise stated. The first diamond in every K-pulse plot is always a unit impulse. This is highlighted by an arrow in the figures. The normalization of using a unit impulse at $t=0$ for the $K$-pulse can be considered as an impulsive excitation of the target by 
the first impulse function. Then, the later part of the K-pulse is added in order to cancel all the resonances. Any other type of desired "excitation" waveform can be convolved with this K-pulse to obtain the . proper K-pulse "excitation" waveform. 


\section{CHAPTER II}

THEORY

In this chapter, the method of estimating the $K$-pulse of the target using the impulse response is described first. Then the pole-extraction scheme is presented.

\section{A. K-PULSE DERIVATION}

The K-pulse as defined by Kennaugh in [2] is, a time-limited waveform of excitation which produces time-limited waveforms of response for all aspects and polarizations. The K-pulse is not unique in form unless it contains only zeros which coincide with the poles of the target [.14]. This is to rule out the possibility that a linear combination of derivatives of the K-pulse as another possible $K$-pulse, as the differentiation process adds zeros which are not poles of the target. The duration of the $K$-pulse being minimum is a necessary condition. The whole approach described in this dissertation is based on the latter part of the definition of the K-pulse; namely, the K-pulse response of the target is time-limited. Assuming the K-pulse as some function, the energy content outside the duraiion of the K-pulse response of the target is minimized with respect to a set of unknowns. The assumed model of the $K$-pulse is

$$
k(t)=\delta(t)+\sum_{n=1}^{N} a_{n} \delta(t-n \tau),
$$


with $\tau=T / N$, where $T$ is the duration of the $K$-pulse; $N$ is the number of delta functions; and the $a_{n}$ 's are unknown real constants. Define $J$, the cost function, to be the energy outside the $K$-pulse response duration; i.e.,

$$
J=\int_{T_{R}}^{T} C[k(t) \star h(t)]^{2} d t ;
$$

where $h(t)$ is the impulse response of the target, $T_{R}$ is the duration of $K$-pulse response, $T_{C}$ is the cutoff time representing an arbitrarily long duration, and " $\star$ " denotes convolution.

By minimizing the cost function $\mathrm{J}$, the coefficients of Equation (2.1) can be found. The reason why impulsive functions are chosen as basis functions is that they are easily evaluated in the convolution integral. Any other basis function requires actual calculation of the convolution integral. Also, the impulses can be treated as samples of a continuous function. Thus, the impulsive representation is fairly general.

The cost function $\mathrm{J}$ can be put into a quadratic form. This will be shown next. The convolution of the K-pulse model with the impulse response is

$$
k(t) \star h(t)=\left[\delta(t)+\sum_{n=1}^{N} a_{n} \delta(t-n \tau)\right] \star h(t) .
$$

Or

$$
\begin{aligned}
& k(t) \star h(t)=h(t)+\sum_{n=1}^{N} a_{n} h(t-n \tau), \\
& \text { because, } \int_{-\infty}^{\infty} \delta(s-n \tau) h(t-s) d s=h(t-n \tau) .
\end{aligned}
$$


The square of Equation (2.4) gives

$$
\begin{aligned}
{[k(t) \star h(t)]^{2} } & =h^{2}(t)+2 h(t) \sum_{n=1}^{N} a_{n} h(t-n \tau) \\
& +\sum_{n=1}^{N} \sum_{m=1}^{N} a_{n} a_{m} h(t-n \tau) h(t-m \tau) .
\end{aligned}
$$

Then the cost function given by Equation (2.2) becomes

$$
\begin{aligned}
J= & \int_{T_{R}}^{T} C n^{2}(t) d t+2 \sum_{n=1}^{N} a_{n} \int_{T_{R}}^{T} C h(t) h(t-n \tau) d t \\
& +\sum_{n=1}^{N} \sum_{m=1}^{N} a_{n} a_{m} \int_{T_{R}}^{T} C(t-n \tau) h(t-m \tau) d t .
\end{aligned}
$$

Matrices are defined to simplify the algebraic manipulations.

The matrices are

$$
\begin{aligned}
& A=\left[\begin{array}{cc}
a_{1} \\
a_{2} \\
\cdot \\
\vdots \\
a_{N} \\
-
\end{array} \mid,\right. \\
& H=\left[\begin{array}{c}
- \\
h(t-\tau) \\
n(t-N \tau) \\
-
\end{array}\right],
\end{aligned}
$$




$$
\Phi=\int_{T_{R}}^{T} C^{\top} H H^{\top} d t,
$$

where $H^{\top}$ is the transpose of $H$,

$$
S=\int_{T_{R}}^{T} C h(t) H d t,
$$

and

$$
\beta=\int_{T_{R}}^{T} C h^{2}(t) d t .
$$

Using the above matrices, the cost function in Equation (2.7) can be expressed as

$$
J=A^{\top} \Phi A+2 A^{\top} S+B,
$$

which is a quadratic form. To find the extremum of a cost function, the gradient with respect to each $a_{n}\left(\nabla_{a}\right)$ is equated to zero; i.e.,

$$
\nabla_{\mathrm{a}} \mathrm{J}=2 \Phi A+2 S=0 .
$$

Thus, A can be evaluated via the following equation:

$$
A=-\Phi^{-1} S \text {, }
$$

where $\Phi^{-1}$ is the inverse of $\Phi$.

In most problems that were considered, $\Phi$ is 111 -conditioned or singular. This is because when $N$ is large, some of the elements in the matrix become very small, especially the elements in the lower right corner. Thus, Equation (2.15) cannot be used directly. Optimization schemes may be used to search for the minimum point of the cost function with respect to the unknowns. The facts that 1) $\mathrm{J}$ can be expressed as a 
quadratic form, 2) $\Phi$ is not a null matrix and 3 )

$$
\nabla_{a}^{2} \mathrm{~J}=2 \Phi,
$$

mean there exists a unique global minimum or maximum point [15]. One good check to determine if $T$ and $T_{R}$ are chosen properly is whether $\Phi$ is semi-positive definite. The optimization schemes employed in this dissertation are conjugate gradient [15], Powell-Fletcher [15] and steepest decent [15]. The former two methods are programmed as subroutines in the IBM System/360 Scientific Subroutine Package version III. All three methods use the same idea of testing for the steepest decent direction and making a step. They only differ in the testing and stepping strategy. The three methods are incorporated so that the methods can be inter-checked for the minimum of the cost function. The Powell-Flectcher scheme seems to converge the fastest. The conjugate gradient scheme is in the middle. The steepest decent scheme is programmed so that the steps are controlled by the user to step cautiously when the minimum is close by.

\section{B. POLE EXTRACTION}

As this approach is minimizing the energy excited by the natural resonances in the signal, the dominant zeros of the $K$-pulse waveform should coincide with the dominant poles of the target. Also, all waveform data manipulated by the computers are usually only samples of the continuous waveforms. Equation (2.1) can be viewed to represent $\delta(t)$ plus samples of a continuous function. To obtain the zeros of the 
continuous function, a straight line interpolation model is assumed between the samples of the continuous function. Then

$$
k_{e}(t)=\delta(t)+k_{c}(t)
$$

where $k_{c}(t)$ is a continuous function with straight lines joining all the $a_{n}$ 's. One such example is shown in figure 2.1a. The reason why straight line interpolation and extrapolation are used is $K_{c}(s)$ can be easily found for the linearly interpolated function $k_{c}(t)$. This is sufficiently accurate provided the sampling criteria is sufficiently satisfied. From experience, if the sampling used is at least four times the required Nyquist rate, then straight line interpolation is adequate. With this straight iline interpolation, the differentiation rule of the Laplace transform can be applfed very conventently to obtain an expression for $K_{c}(s)$ which is the Laplace transform of the continuous portion of the $k$-pulse $k_{c}(t)$. Then the zeros of the $k_{e}(s)$ can be obtained via some zero-finding numerical subroutines. Naturally other types of interpolation can be applied. This might reduce the number of samples required to approximate the continuous portion of the K-pulse. However this is the same as expanding the continuous portion of the K-pulse in a set of basis function. Unless there are some indications as to what the K-pulse might look like, there does not seem to be much advantages with this alternate approach. Besides, even with a continuous function, the computer can only work with the samples of the function.

Differentiating the linearly interpolated and extrapolated $k_{c}(t)$ (Figure 2.1b), 


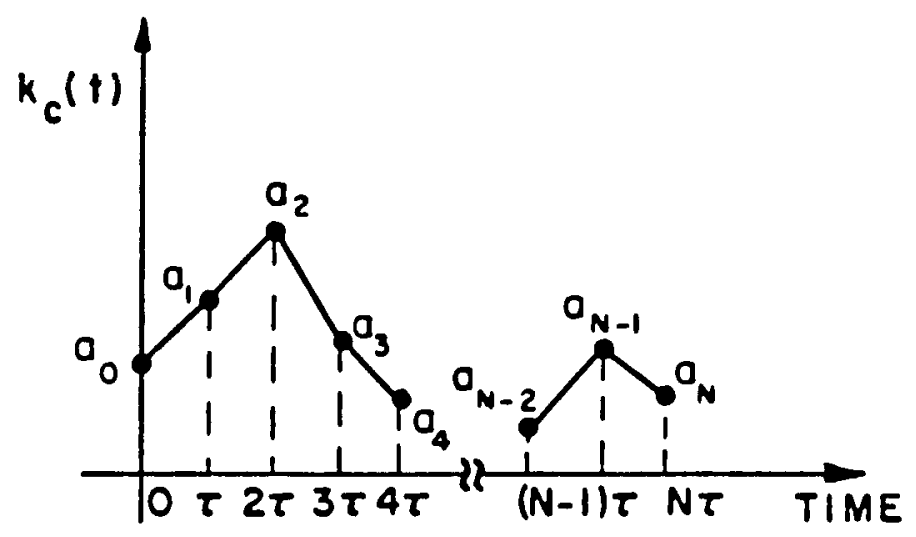

(a) Iinear interpolation and extrapolation $\left(a_{0}\right)$ of samples.

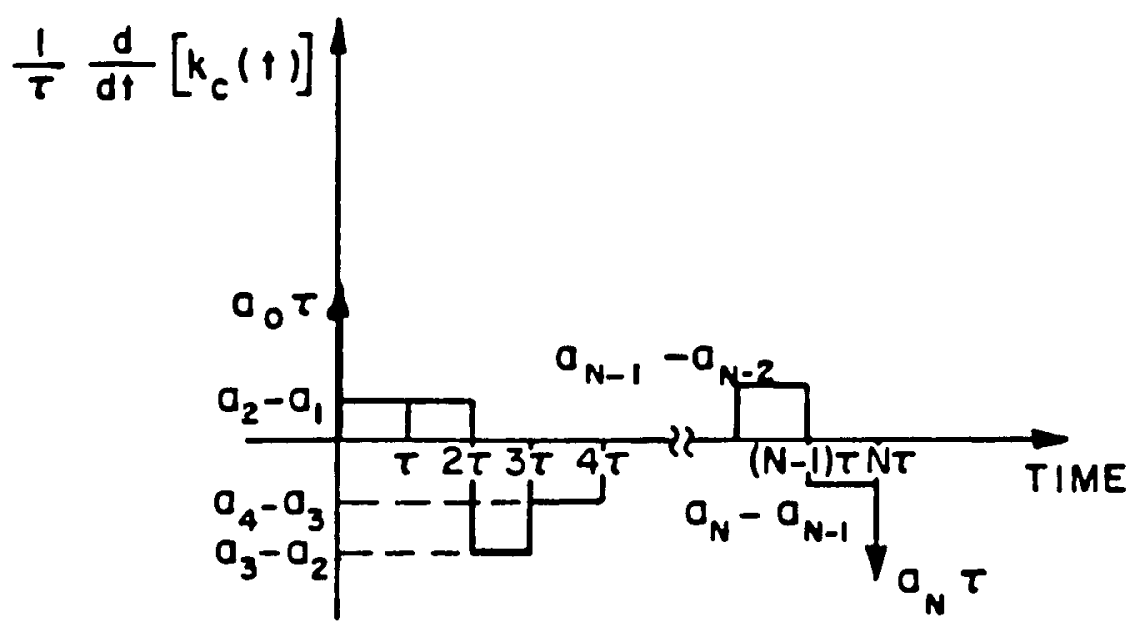

(b) The derivative of the top figure.

Figure 2.1. An fllustration of linear interpolation and extrapolation $\left(a_{0}\right)$ of samples and their derivatives. 


$$
\begin{aligned}
\frac{d}{d t} k_{c}(t) & =a_{0} \delta(t)-a_{N} \delta(t-T) \\
& +\frac{1}{\tau} \sum_{n=1}^{N}\left(a_{n}-a_{n-1}\right) p_{\tau / 2}\left[t-\left(\frac{2 n-1}{2}\right)_{\tau}\right],
\end{aligned}
$$

where the $a_{n}$ 's are samples of the continuous function for $n=1,2$, $\ldots, N ; a_{0}$ is the linear projection from $a_{1}$ and $a_{2} ; p_{\tau / 2}(t)$ is the rectangular pulse function [16]; $T$ is the duration of the K-pulse. By taking the Laplace transform of Equation (2.18),

$$
\mathcal{L}\left[\frac{d}{d t} k_{c}(t)\right]=a_{0}-a_{N} e^{-s T}+\left[\frac{1-e^{-s \tau}}{s \tau}\right] \sum_{n=1}^{N}\left(a_{n}-a_{n-1}\right) e^{(1-n) s \tau}
$$

Or

$$
\mathscr{L}\left[\frac{d}{d t} k_{c}(t)\right]=s k_{c}(s) .
$$

Then, the K-pulse spectrum is

$$
K_{e}(s)=1+K_{c}(s)
$$

or

$$
k_{e}(s)=1+\frac{a_{0}-a_{N} e^{-s T}}{s}+\left[\frac{1-e^{-s \tau}}{s^{2} \tau}\right] \sum_{n=1}^{N}\left(a_{n}-a_{n-1}\right) e^{(1-n) s \tau}
$$

The complex frequencies $\left(s_{i}\right)$ which satisfy $K_{e}\left(s_{j}\right)=0$, are the zeros of the K-pulse. These, in turn, are also the poles of the target. The roots of Equation (2.22) are numerically evaluated using the Secant method [17]. 
CHAPTER III

APPLICATION TO THEORETICAL DATA

In this chapter, the theory in the previous chapter is applied to theoretical data. There are altogether seven examples. A plane wave normally incident on a grounded dielectric slab was one of the examples discussed in Kennaugh's paper [2] to demonstrate the K-pulse. This target is also employed in this dissertation to fllustrate the validity of the technique described in the last chapter. First, the result of using the exact impulse response of the grounded dielectric slab is presented. Then the technique is checked with band-limited data. A Fourier synthesized impulse response for the same grounded dielectric slab is used as a second example. The words "Fourier synthesized" implies that the impulse response data are generated in the frequency domain first, then a discrete Fourier transformation is used to obtain the data in the time domain. A Kaiser-Bessel function [18] is used to weight the frequency data before transformation to reduce the Gibb's phenomena. The third example is a Fourier synthesized impulse response of a low $Q$ model suggested by Kennaugh [19]. This example is to illustrate that if the impulse response at late time contains contributions from a lot of the poles, then even for a low $Q$ structure, the K-pulse can be approximated very nicely. The exact K-pulse for the previous three examples are known, that is why they are presented first. The exact $K$-pulses for the last four examples in this chapter are 
unknown, thus the K-pulses obtained can only be checked by their zero strings and aspect-independent property. The last examples are all three dimensional simple geometries (see Figure 3.1). (For the convenience of the reader, all tables and figures of Chapter III are grouped together at the end of the chapter.) The results of two high $Q$ structures (a thin conducting straight wire and a thin conducting circular loop) are presented next. Finally, two low 0 structures (a conducting sphere and a conducting disk) are used as examples.

\section{A. EXAMPLE I}

A plane wave is normally incident on a grounded dielectric slab. The grounded dielectric slab has a thickness of $L$ and a relative permittivity of four. All diamonds in Figures $3.2,3.3,3.4$ and 3.5 represent impulses. The impulse response of the grounded dielectric slab is shown in Figure 3.2. As shown in Figure 3.2, the target response is resonating with decaying positive and negative impulses. The target response is desired to have a finite duration. To start the optimization process, twenty-six unit impulses are used as initial guesses for the K-pulse. Since any initial guess of the unknowns is as good as any value, one is chosen to be such value. The duration of the $K$-pulse (T) is chosen as $2 L / v$, which is the round trip time to and from the ground plane. " $v "$ is the speed of light travelling in the dielectric slab. The response of the grounded dielectric slab to these impulses is shown in Figure 3.3. This inftial K-pulse gives rise to more resonating impulses (see figure 3.3 ) than the impulse response 
(Figure 3.2). The impulse at $2 \mathrm{~L} / \mathrm{V}$ is contributed by the input impulses at $t=0$ and $t=2 L / v$ together. All the impulses after $2 L / v$ are undesirable. As a result, the duration of the $k$-pulse response $\left(T_{R}\right)$ and the cutoff time of integration $\left(T_{C}\right)$ are chosen to be $2.04 \mathrm{~L} / \mathrm{V}$ and $12 \mathrm{~L} / \mathrm{v}$, respectively. After specified number of optimization steps, the K-pulse results as in Figure 3.4; the K-pulse response is shown in Figure 3.5. (Appendix A contains a more detall discussion on the optimization process and the convergent indicators.) The weightings of all twenty-six unit impulses in the initial K-pulse, except two, are reduced to zero. The combination of the two remaining impulses, one at $t=0$ and one at $t=2 L / v$ converges to $\delta(t)+(1 / 3) \delta(t-2 L / v)$, which is the exact K-pulse [2] for this geometry. All the undesirable resonating impulses in the response due to the initial K-pulse guess are eliminated in the optimization process as shown in Figure 3.5. All, except two, of the impulses in the optimized K-pulse response, are reduced numerically to zero. The remaining impulse at $t=0$ has a value of $-1 / 3$ and the other impulse at $t=2 L / v$ has a value of -1 . This $K$-pulse response converges to $-(1 / 3) \delta(t)-\delta(t-2 L / v)$, which is the exact $K$-pulse response [2]. Two other initial guesses for the K-pulse as shown in Figure 3.6 are also employed to start the optimization process. The same K-pulse and $K$-pulse response as in Figures 3.4 and 3.5 are obtained. This further supports the fact that this theory is independent of the initial K-pulse guess supplied to start the optimization process. 
The plane wave normally incident on a grounded dielectric slab can be put into a transmission line model [2]. The model consists of a shorted length $(L)$ of line which is appended to a semt-infinite line of twice the intrinsic impedance. The resulting transfer function is given by

$$
H(s)=\frac{-\frac{1}{3}-e^{-2 s L / c}}{1+\frac{1}{3}-e^{-2 s L / c}}
$$

Frequency data are generated with the above expression, over the frequency band of $k L=0.01 \pi(0.01 \pi) 100 \pi$, where $k$ is the wave number. The notation employed for the bandwidth is: 1) the first number is the lowest frequency used, 2) the second number which is in brackets is the frequency increment and 3) the last number is the highest frequency used. The Fourier synthesized impulse response of the grounded dielectric slab is shown in Figure 3.7 for a duration of $6 \mathrm{~L} / \mathrm{v}$. Its amplitude has been reduced by a factor of ten after the discrete Fourier transform process. Since the impulse response is bandlimited, the impulses in Figure 3.2 now have finite rise and fall times. The oscillating impulses after $2 L / V$ are still the important features in Figure 3.7. The duration of the K-pulse is chosen as $2 \mathrm{~L} / \mathrm{V}$. The duration of the $K$-pulse response is chosen as $2.05 \mathrm{~L} / \mathrm{v}$. The reason why the $K$-pulse response duration is not chosen as $2 \mathrm{~L} / \mathrm{V}$ is to account for the finite rise and fall time of the bandlimited impulse. The cutoff time for the integration is $8 \mathrm{~L} / \mathrm{V}$. Eleven unit impulses are used to start the optimization process. The response due to this initial input 
is shown in Figure 3.8. This figure shows a lot of resonating impulses after $2.05 \mathrm{~L} / \mathrm{v}$. The approximate $K$-pulse obtained after optimization is shown in Flgure 3.9. Again, only two impulses remain numerically significant after optimization - the unit impulse at $t=0$ and the 0.3328535 impulse at $t=2 L / v$. The percentage of difference is $0.14 \%$ for the impulse at $t=2 L / v$. The optimized $K$-pulse response, which is shown in Figure 3.10, has only a small residue after $t=2.11 / v$. Although only a duration of $6 \mathrm{~L} / \mathrm{v}$ is shown in Figure 3.10 , the amplitude of the signal at later times is negligible. This response has a duration of $2.1 \mathrm{~L} / \mathrm{v}$ instead of $2 L / v$ because the bandwidth of the impulse response is limited. However, the time between the peaks of the first two Fourier synthesized impulses is still $2 \mathrm{~L} / \mathrm{v}$. The poles associated with the approximate $K$-pulse are $-1.1001+(2 n+1) \pi$ versus $-1.0986+(2 n+1) \pi$ from the exact $K$-pulse, where $n$ is an integer. These poles are normalized to $2 L / v$; $1 . e .$, to obtain the actual values of the natural resonances, multiply the above pole values by twice the thickness of the slab (L) and divide by the speed of light in the dielectric medium.

\section{EXAMPLE III}

The following expression [19] is used as a first check on the concept of using impulses to represent samples of a continuous waveform:

$$
H(s)=\frac{(s L / c)^{2}}{-1+(s L / C)+(s L / C)^{2}+\exp (-s L / C)}
$$

This $H(s)$ can be decomposed into a fraction of two entire functions [2]. Using l'Hopital's rule, $H(s)$ can be proven not to have any zero. Since 
$H(s)$ has no zero, it is decomposed into $R(s)$ which is the $K$-pulse response in the s-domain,

$$
R(s)=1 \text {, }
$$

and $K(s)$ which is the complex frequency spectrum of the $K$-pulse,

$$
K(s)=\frac{\exp (-s L / c)-1}{(s L / c)^{2}}+\frac{1}{(s L / c)}+1 \text {, }
$$

which is also the reciprocal of $H(s)$.

Inverse Laplace transforming Equation (3.4), the K-pulse in the time domain consists of a delta function at $t=0$ and a linear decaying. function of duration $L / C$. The maximum value of the decaying function is one. The synthesized impulse response $[h(t)]$ is obtained via fast Fourier transform on the data generated from Equation (3.2). The bandwidth used to generate this waveform is $k L=0.2 \pi(0.2 \pi) 400 \pi$. The number of samples used to approximate the continuous portion of the $K$-pulse $\left[k_{c}(t)\right]$ is 200 . The duration of the $K$-pulse is chosen to be $\mathrm{L} / \mathrm{c}$. The duration of the $\mathrm{K}$-pulse response is chosen as $0.025 \mathrm{~L} / \mathrm{C}$, since an Impulse function [Equation (3.3)] now has a finite rise and fall time due to bandlimited data. The cutoff time for integration is $2 L / C$. After optimization, samples of the optimized K-pulse are plotted against the exact $K$-pulse in Figure 3.11. The first diamond at $t=0$ is representing an impulse, which is highlighted by an arrow; the rest of the diamonds are representing samples of the continuous portion of the $K$-pulse $\left[k_{c}(t)\right]$. It can be seen that the optimized K-pulse gives a very good approximation to the exact K-pulse. The Fourier synthesized impulse response generated with Equation (3.2) and the optimum K-pulse 
response are shown in Figure 3.12. The energy contained in the K-pulse response is almost limited only to the Fourier synthesized impulse. The energy that spread over infinity in the impulse response is reduced almost to zero. This energy spread can also be considered as the energy contributed by the infintte pole string. Thus, the pole-zero cancellation concept is established. Table 3.1 lists the first ten poles from Equation (3.2) and first ten zeros of Equation (2.22) with the proper $a_{n}$ 's from the optimized K-pulse for comparison. The zeros and the poles are both normalized to $L / C$. The zeros of the estimated K-pulse are very close to the exact poles of the frequency response function $H(s)$. This example shows that even for low $Q$ targets, if the impulse response at late time contains sufficient contributions from the higher order poles, the exact K-pulse can be approximated fairly well. Starting with the next example, the exact $k$-pulse is no longer known.

\section{EXAMPLE IV}

A conducting straight wire of length $L$ with length to radius ratio of 2000 , is chosen as the next example. The frequency response data are generated from a moment method solution [20]. The bandwidth of the responses is $\mathrm{KL}=0.08 \pi(0.08 \pi) 8 \pi$. After discrete Fourier transforming the frequency data, the maximum magnitude of the impulse responses are normalized to one. All data in this example are taken at $\theta=90^{\circ}$, ф-polarization. The geometries are shown in Figure 3.1. The K-pulse in Figure 3.13 is derived from the Fourter synthesized impulse response at 
$\phi=30^{\circ}$. The duration of the $\mathrm{K}$-pulse is chosen to be $2 \mathrm{~L} / \mathrm{C}$. The energy between $5 L / C$ and $9 L / C$ is being minimized. The number of samples chosen for the continuous portion of the $k$-pulse $\left[k_{c}(t)\right]$ is 100 . The initial guesses for the samples are unity. The impulse and the K-pulse responses of the wire at $\phi=30^{\circ}$ are compared in Figure 3.14. The high energy resonance after $4 L / C$ has been greatly reduced. It is also interesting to note that the duration of the $K$-pulse response does not have to be known exactly to obtain the K-pulse, as the optimization in this example is specified to perform minimization beginning at $5 \mathrm{~L} / \mathrm{C}$ instead of $4 L / C$, which is the more accurate estimation of the K-pulse response duration. The aspect independence of the K-pulse obtained can be shown by convolving the obtained K-pulse with other aspect angles. Figures 3.15 and 3.16 show the comparison of the impulse and the $K$-pulse responses of the wire at $\phi=60^{\circ}$ and $90^{\circ}$, respectively. At $\phi=60^{\circ}$, the $K$-pulse response shows very little energy after $3 \mathrm{~L} / \mathrm{C}$. At $\phi=90^{\circ}$, the K-pulse response indicates very $1 \mathrm{fttle}$ resonance after $2.6 \mathrm{~L} / \mathrm{c}$. The reason why at broadside the $K$-pulse does not terminate at $2 \mathrm{~L} / \mathrm{C}$ is again due to the limited bandwidth of the data. It takes finite time to start and end a signal.

Initially, the number of samples (N) employed is 50 . The increase of sampling from 50 to 100 does not have a drastic effect on the shape of the K-pulse. Moreover, the accuracy of the poles obtained from $\mathrm{N}=50$ improves when $N$ is increased to 100 ; the energy content outside the K-pulse response also decreases. All these are indicators that the K-pulse obtained is stepping in the right direction. If the number of 
samples $(N)$ is reduced to less than 50 in this case, one should watch out for the violation of the criterion for straight line interpolation of the samples. The poles obtained with the K-pulse in Figure 3.13 are compared with those obtained by Kennaugh [19] in Table 3.2. The poles are normalized to $L / C$. The poles are more accurate in the imaginary parts than the real parts. This thin wire example was also discussed by Kennaugh [2]. The $K$-pulse that was shown in [2] is not time-limited; whereas, Figure 3.13 is time-limited. It is believed that Prof. Kennaugh only intended to illustrate that if the $K$-pulse is ever needed for actual excitation of targets, it can be approximated by impulsive and step functions. The pole-zero cancellation concept is still embedded in his $K(s)$.

\section{E. EXAMPLE V}

The second high $Q$ geometry to be examined, is the conducting circular loop with wire radius (a) to loop radius (r) ratio of $\pi \times 10^{-3}$. The frequency data are generated using a moment method program [10] over the bandwidth $\mathrm{kr}=0.02 \pi(0.02 \pi) 4 \pi$. After these frequency data are discrete Fourier transformed into the time domain, the maximum magnitude of the impulse responses are normalized to one. The $K$-pulse in Figure 3.17 is derived from the Fourter synthesized impulse response at $\theta=45^{\circ}$, $\hat{\theta}$-polarization. The geometries are shown in Figure 3.1. The duration of the K-pulse (T) is chosen to be $2 \pi r / c$. The number of samples (N) to 
represent the continuous portion of the K-pulse is 100 . The duration of the K-pulse response is chosen to be $5 \pi r / c$. After optimization, the resulting $K$-pulse response has $1 f t t l e$ energy after $4 \pi r / c$. This is contrasted with the high oscillation of the impulse response in figure 3.18. Again even though the exact $K$-pulse response duration is not supplied for minimization of energy, the proper $K$-pulse response duration shows up in Figure 3.18 to be $4 \pi \mathrm{r} / \mathrm{C}$ after optimization. The convolution of the approximate K-pulse with three other impulse responses $-\theta=0^{\circ}, \hat{\phi}$-polarization; $\theta=45^{\circ}, \hat{\phi}$-polarization; $\theta=90^{\circ}$, $\hat{\phi}$-polarization are shown in Figures $3.19,3.20$ and 3.21 respectively. Again, the respective impulse responses are also plotted in the same figures using dash lines. The energy content in the $K$-pulse responses at late time $-2.4 \pi r / c$ in Figure $3.19 ; 4 \pi r / c$ in Figures 3.20 and 3.21 , is very small. Again the aspect and polarization independent property of the $K$-pulse is confirmed. In fact, the initial trys of optimization using $N=50$ for $\theta=45^{\circ}$, both $\hat{\theta}$ and $\hat{\phi}$ polarizations gave similar $K$-pulses (see Appendix A). This is only true, if similar poles are excited by the two different impulse responses.

The poles obtained from the $K$-pulse is compared with the poles given by Kim et al. [10] in Table 3.3. These poles are normalized to $2 \pi r / c$. The real parts of the estimated poles are not as accurate at the high frequency end as the ones at the low frequency. This is probably due to the limited bandwidth of the impulse response used. However the imaginary parts are consistently accurate. As $N$ is increased, similar properties described previously in Example IV about the similarity of 
K-pulse shapes, better accuracy of the poles and reduction of energy content outside the duration of $K$-pulse response are also exhibited by the loop. Kim et al. [10] also obtained a K-pulse for the circular loop with the same geometry. However, it is very difficult to compare with their result. Their $K$-pulse is bandlimited. While the K-pulse described here itself is not bandlimited, the impulse response it used is bandlimited. Also, if not all the poles are excited at late time in the impulse repsonse (due to limited bandwidth), then the $K$-pulse obtained here cannot claim uniqueness. Nevertheless, the duration of the K-pulse responses at the different aspects and polarizations are very close to Kim et al.'s [10] results. Appendix $C$ contains a more detail discussion on the two $\mathrm{K}$-pulse models. The next two examples involves low $Q$ structures.

\section{F. EXAMPLE VI}

The first low 0 geometry to be discussed is the conducting sphere. The frequency data used in this example are generated from the Mie solution. Since this is a low 0 structure, the choice of $T_{R}$ (the K-pulse response duration) must be chosen very carefully. If too large a value is chosen, the energy content to be minimized will be very small and only the very dominant poles will be excited. If too small a value is chosen, the energy to be minimized may contain contributions other than the poles - the entire function in the early time [21], resulting in wrong pole values. The K-pulse in Figure 3.22 is obtained from the backscattered Fourier synthesized impulse response generated with a 
frequency bandwidth of $k r=0.1(0.1) 66$. Note that " $r$ " is the radius of the sphere. The duration of the K-pulse $(T)$ is chosen to be $2 \pi r / c$. The duration of the K-pulse response is about $3.8 \pi \mathrm{r} / \mathrm{c}$. The number of samples to approximate the continuous portion of the K-pulse is 200 . Figure 3.23a compares the $K$-pulse and the impulse responses of the sphere for the backscattered case. Since the structure has a low 0 , the effect of the K-pulse on the small resonance cannot be seen clearly in Figure 3.23a. The amplitude scale in Figure $3.23 \mathrm{~b}$ is 100 times that of Figure 3.23a. All the amplitude scales in the "b" designated figures are expanded 100 times relative to those of the "a" designated figures in the following discussions, unless otherwise stated. Figures 3.24 through 3.27 are generated from a bandwidth of $k r=0.1(0.1) 33.5$. Figure 3.24 is the same as Figure 3.23 , except these backscattered data have a smaller bandwidth. Figures 3.25 and 3.26 use data from bistatic scattering at $\phi=90^{\circ}$, vertically and horizontally polarized, respectively. Figure 3.27 uses bistatic scattering at $\phi=180^{\circ}$, for vertically polarized data. Again, at late time $-3.8 \pi r / \mathrm{C}$ in Figure $3.24,4.2 \pi r / C$ in Figures 3.25 and $3.26,4 \pi r / C$ in Figure 3.27 , the energy content is smaller than those in the impulse responses.

The zeros of the K-pulse in Figure 3.22 is compared with Kennaugh's result [2] in Table 3.4. The poles are normalized to $2 \pi r / c$. The first six poles compare well; whereas, the higher order poles do not. Using the contribution of residues and poles discussed by Chen [22] to compare with the late time signal of Figure $3.23 \mathrm{~b}$ beginning at $3.6 \mathrm{wr} / \mathrm{c}$, the contribution of the first six pairs of poles and residues seems 
sufficient to represent the late time function. This suggests that the method discussed in this dissertation can obtain the poles only if they are excited. Or the $K$-pulse for this example is not unique, until the other poles of the sphere are included. Actually, applying this method to a low $Q$ structure is not very useful, as this method requires a minimization of energy content outside the duration of the K-pulse response. And there is very little energy in the late time of the impulse response of a low $Q$ structure to be manipulated. Kennaugh [2] suggested a better method to obtain the poles of the low Q structures using the Geometrical Theory of Diffraction [23].

\section{G. EXAMPLE VII}

The last theoretical example to be discussed is the conducting circular disk. The frequency data are generated from an eigenfunction solution [24] over a bandwidth of $k r=0.16(0.16) 16$. In this case, $r$ is the radius of the disk. After discrete Fourier transform is performed on these frequency data, the impulse responses are normalized to a maximum magnitude of one. The $K$-pulse shown in Figure 3.28 is obtained by minimizing the $K$-pulse response $\left(\theta=45^{\circ}\right.$ and $\hat{\theta}$-polarized) outside $4 \pi r / c$. The number of samples (N) used to represent the continuous portion of the K-pulse is 100 . Figures $3.29,3.30,3.31$ and 3.32 show the comparison between the impulse and $K$-pulse responses of the disk at $\theta=45^{\circ}, \hat{\theta}$-polarized; $\theta=0^{\circ}, \hat{\theta}$-polarized; $\theta=45^{\circ}, \hat{\phi}$-polarized; and $\theta=90^{\circ}$, $\hat{\phi}$-polarized, respectively. Again the amplitude scales of the "b" designated plots are 100 times those of the "a" designated plots. The 
durations of the $K$-pulse responses are around $4 \pi r / c, 2.4 \pi r / c, 4 \pi r / c$ and $4 \pi r / C$ for Figures $3.29,3.30,3.31$ and 3.32 , respectively. The energy contained outside the $K$-pulse response is very small. If the impulse response at $\theta=45^{\circ}, \phi-p o l a r i z a t i o n$ is used to obtain the $K$-pulse, Figure 3.33 is the resulting $K$-pulse with the number of sampling still at 100 . The responses of the disk to this $K$-pulse at $\theta=45^{\circ}, \hat{\phi}$-polarization; $\theta=0^{\circ}, \hat{\theta}$-polarization; $\theta=45^{\circ}, \hat{\theta}$-polarization; $\theta=90^{\circ}, \hat{\phi}$-polarization are shown in Figures $3.34,3.35,3.36$ and 3.37 , respectively. The results of this K-pulse are compatible with the K-pulse derived from the $\theta=45^{\circ}$, and $\hat{\theta}$-polarization. The energy content outside the $K$-pulse responses duration is very small. The reason for the two different $K$-pulses is as discussed in the previous example. There are two sufficient conditions for two different K-pulses from two different impulse responses of the same target. The first condition is insufficient pole-excitation in the late time. The second condition is different poles are excited in the impulse responses. Both conditions seem to be satisfied in this case, as discussed in the following paragraph. Consequently, it is not easy to distinguish low 0 targets using the energy content at late time, but the dominant complex natural resonances are still very useful information.

The poles of the two K-pulses are listed together with the poles extracted by Lee [25] in Table 3.5. The poles are normalized to $2 \pi r / c$. Starting with the sixth pole for the first K-pulse (Figure 3.28) and the fifth pole for the second K-pulse (Figure 3.33), the poles do not seem to match. In fact, the real parts do not follow the same trend as 
obtained by Lee. The same behaviour was exhibited in the previous example. The explanation is insufficient pole excitation at late time. Since the disk is a low 0 structure, not all poles are expected to contribute at late time. The fourth poles of the first K-pulse ( $\hat{\theta}$-polarization) seems to be out of place. Actually, this pole compares fairly well with the first pole listed by Lee [25] as the broadside pole $--6.535+31.757 j$. This suggests that $\hat{\theta}$-polarization at $\theta=45^{\circ}$ excites the broadside pole string, but not the $\hat{\phi}$-polarization at $\theta=45^{\circ}$. Consequently, two different K-pulses are obtained for the different polarizations at $\theta=45^{\circ}$. Comparing Figures $3.29 \mathrm{~b}$ through $3.32 \mathrm{~b}$ with 3.34b through $3.37 b$, the K-pulse derived from $\hat{\theta}$-polarization (Figure 3.28) seems to perform better in minimizing the energy outside the duration of the $K$-pulse responses. 
Table 3.1

A Comparison of the First Ten Exact Poles and Estimated Zeros in the s-domain (Upper Left Half Plane) for the Simulated Model in Equation (3.2)

Exact Poles

1) $-3.6326+5.2336 j$

2) $-5.0874+11.8318 j$

3) $\quad-5.8978+18.2771 \mathrm{j}$

4) $-6.4689+24.6586 j$

5) $-6.9115+31.0086 j$

6) $-7.2733+37.3405 j$

7) $-7.5795+43.6610 \mathrm{j}$

8) $-7.8448+49.9738 j$

9) $-8.0791+56.2811 \mathrm{j}$

10) $-8.2887+62.5843 j$
Estimated Zeros

$-3.6339+5.2321 j$

$-5.0887+11.8302 j$

$-5.8993+18.2749 j$

$-6.4708+24.6557 j$

$-6.9139+31.0050 j$

$-7.2764+37.3360 j$

$-7.5832+43.6556 j$

$-7.8493+49.9675 j$

$-8.0842+56.2740 j$

$-8.2952+62.5769 j$ 
Table 3.2

A Comparison of the First Six Exact Poles and Estimated Zeros

in the s-domain (Upper Left Half Plane)

for the Conducting Thin Straight Wire

$\begin{array}{lcc} & \text { Exact Poles } & \text { Estimated Zeros } \\ \text { 1) } & -0.161+3.013 j & -0.183+2.962 j \\ \text { 2) } & -0.219+6.133 j & -0.278+6.058 j \\ \text { 3) } & -0.262+9.256 j & -0.344+9.180 j \\ 4) & -0.294+12.383 j & -0.393+12.314 j \\ \text { 5) } & -0.320+15.512 j & -0.423+15.448 j \\ \text { 6) } & -0.343+18.642 j & -0.482+18.591 j\end{array}$


Table 3.3

A Comparison of the First Ten Exact Poles and Estimated Zeros in the s-domain (Upper Left Half Plane) for the Conducting Thin Circular Loop

Exact Poles
1)
$-0.423+6.509 j$
$-0.606+12.871 j$
3) $-0.745+19.211 j$
4) $-0.862+25.541 j$
5) $\quad-0.965+31.864 j$
6) $-1.059+38.183 j$
7) $-1.145+44.499 j$
8) $-1.226+50.812 j$
9) $-1.302+57.124 j$
10) $-1.374+63.434 j$
$-0.474+6.509 j$
$-0.674+12.891 j$
$-0.796+19.248 j$
$-0.949+25.560 j$
$-1.055+31.892 j$
$-1.318+38.803 j$
$-1.548+45.326 j$
$-1.786+51.702 j$
$-1.977+58.019 j$
$-2.124+64.319 j$

Estimated Zeros 


\section{Table 3.4}

A Comparison of the First Ten Exact Poles and Estimated Zeros in the s-domain (Upper Left Half Plane) for the Conducting Sphere

Exact Poles

1)

2)

3)

4)

5)

6)

7)

8)

9)

10)

$$
-3.142+5.441 j
$$$$
-4.411+11.354 j
$$$$
-5.297+17.328 j
$$$$
-5.994+23.342 j
$$$$
-6.585+29.380 j
$$$$
-7.094+35.450 j
$$$$
-7.546+41.532 j
$$$$
-7.961+47.627 j
$$$$
-8.338+53.740 j
$$$$
-8.683+59.860 j
$$

Estimated Zeros

$-3.098+5.447 j$

$-4.013+11.444 j$

$-5.204+18.016 j$

$-6.002+24.258 j$

$-6.756+30.077 j$

$-6.908+35.622 j$

$-6.656+41.562 j$

$-6.473+47.785 j$

$-6.382+54.081 j$

$-6.331+60.400 j$ 
Table 3.5

\begin{abstract}
A Comparison of the Estimated First Ten Poles
in the s-domain (Upper Left Half Plane)

for the Conducting Circular Disk
\end{abstract}

$\begin{array}{lccc} & \text { Lee } & \text { First K-pulse } & \text { Second K-pulse } \\ \text { 1) } & -3.178+7.595 j & -3.074+7.685 j & -3.036+7.612 j \\ \text { 2) } & -3.789+14.339 j & -3.941+14.471 j & -3.931+14.425 j \\ \text { 3) } & -4.239+20.934 j & -4.434+20.923 j & -4.386+21.158 j \\ 4) & -4.605+27.462 j & -6.405+28.179 j & -4.533+27.781 j \\ \text { 5) } & -4.918+33.949 j & -4.959+33.996 j & -4.035+33.880 j \\ \text { 6) } & -5.194+40.410 j & -4.929+43.121 j & -4.030+40.762 j \\ 7) & -5.442+46.850 j & -4.962+49.949 j & -4.154+47.317 j \\ 8) & -5.668+53.276 j & -4.355+57.046 j & -4.259+53.785 j \\ 9) & -5.877+59.690 j & -4.364+64.109 j & -4.372+60.208 j \\ \text { 10) } & -6.072+66.095 j & -4.503+70.886 j & -4.467+66.600 j\end{array}$



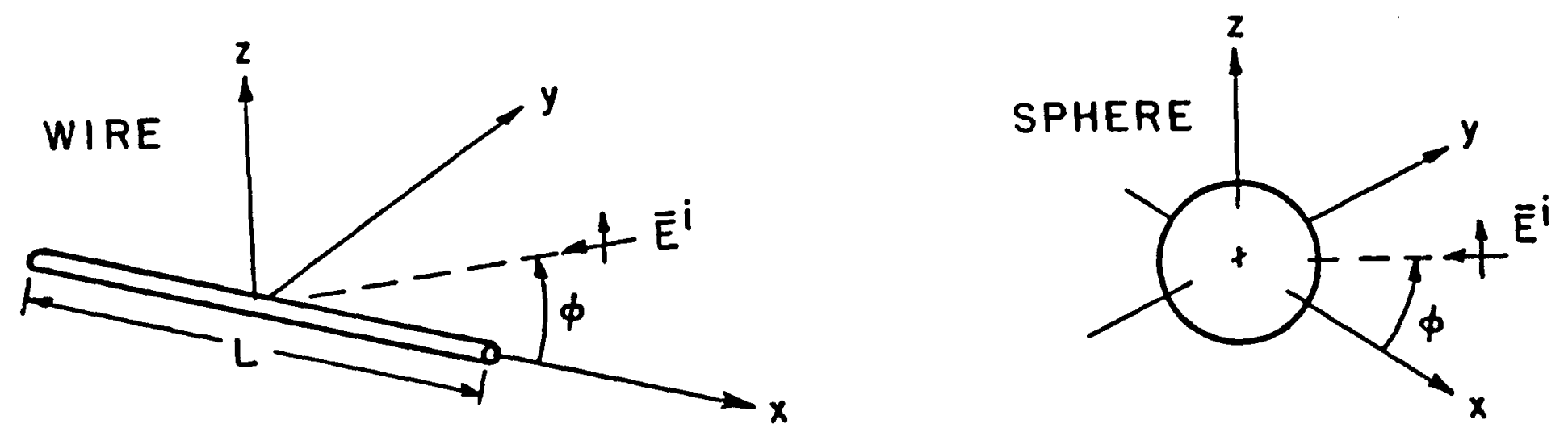

$\stackrel{\omega}{\Phi}$
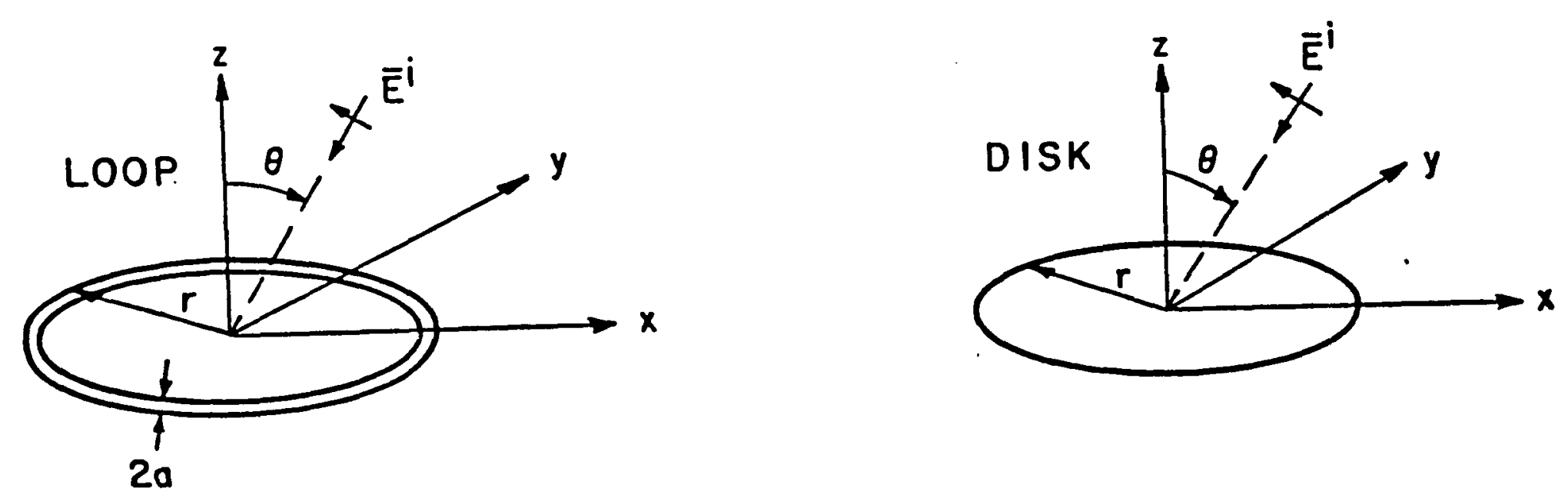

Figure 3.1. The simple target models used to illustrate the method of K-pulse estimation. The orientation genmetry is also given. 


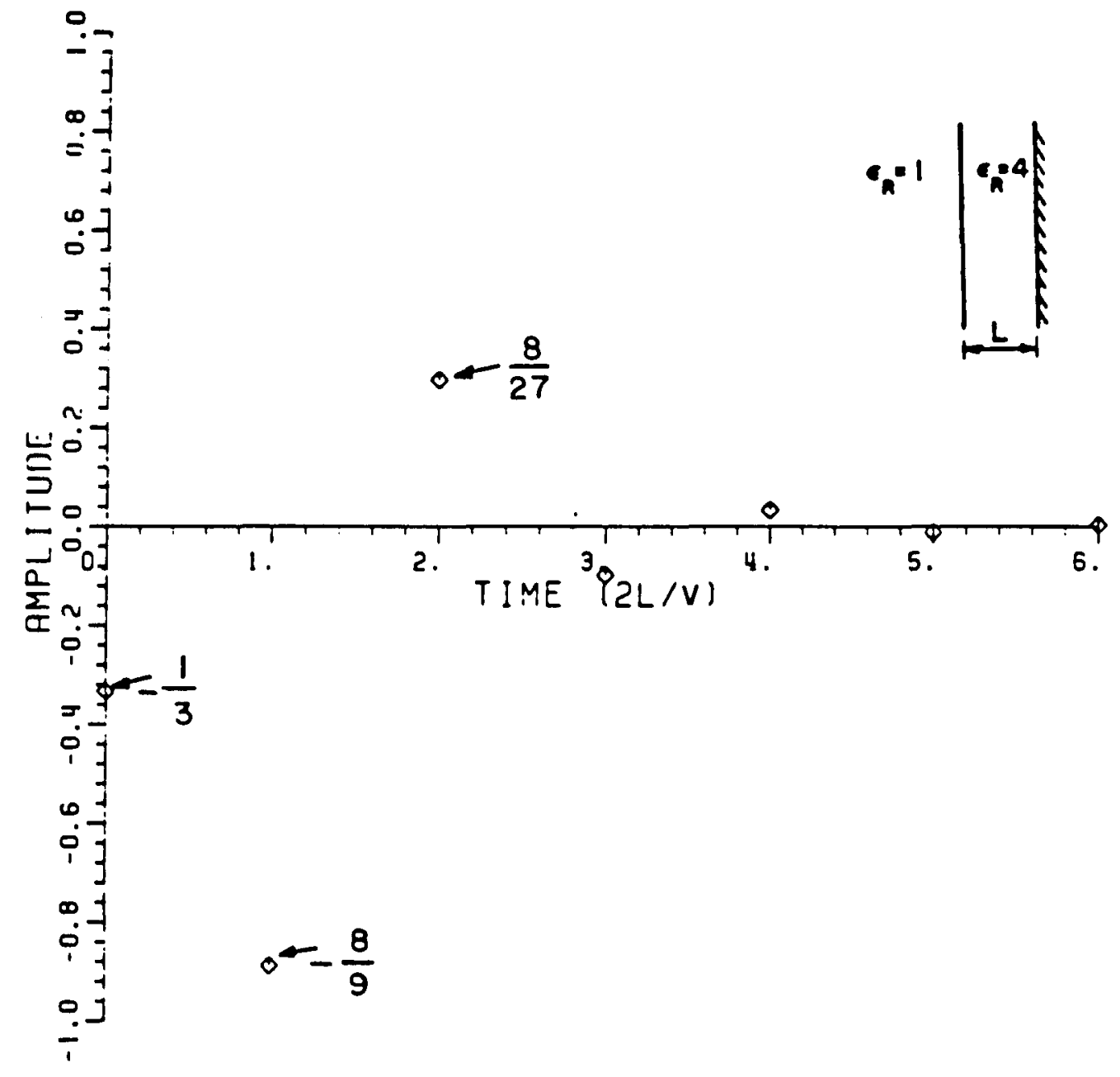

Figure 3.2. Response of the grounded dielectric slab to a normally incident plane wave with impulsive time dependence. (each diamond's height represents the weighting of an impulse) 


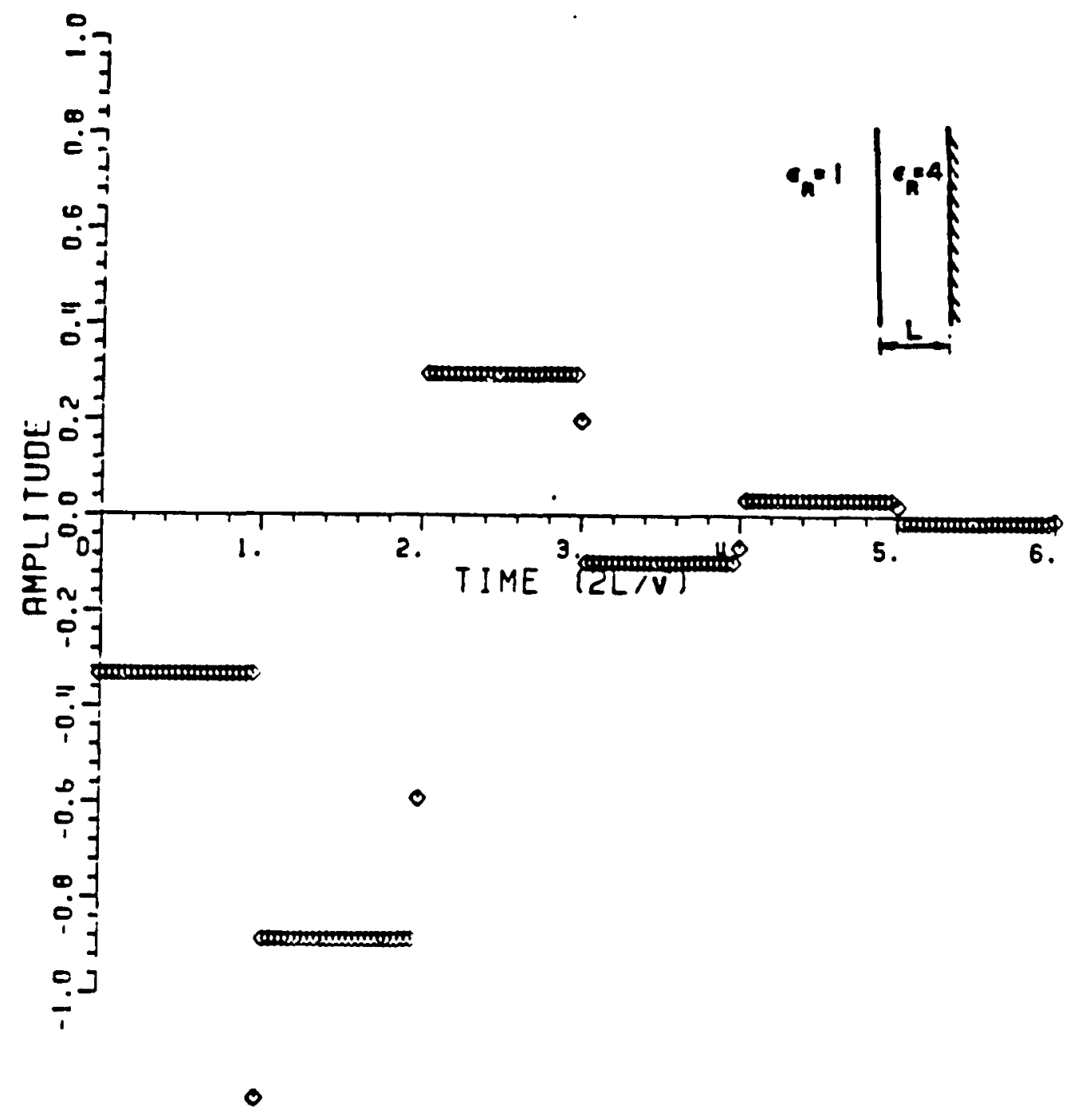

Figure 3.3. Response of the grounded dielectric slab to a set of unit impulses (26) which represents the first guess for the $K$-pulse. (each diamond's height represents the weighting of an impulse). 


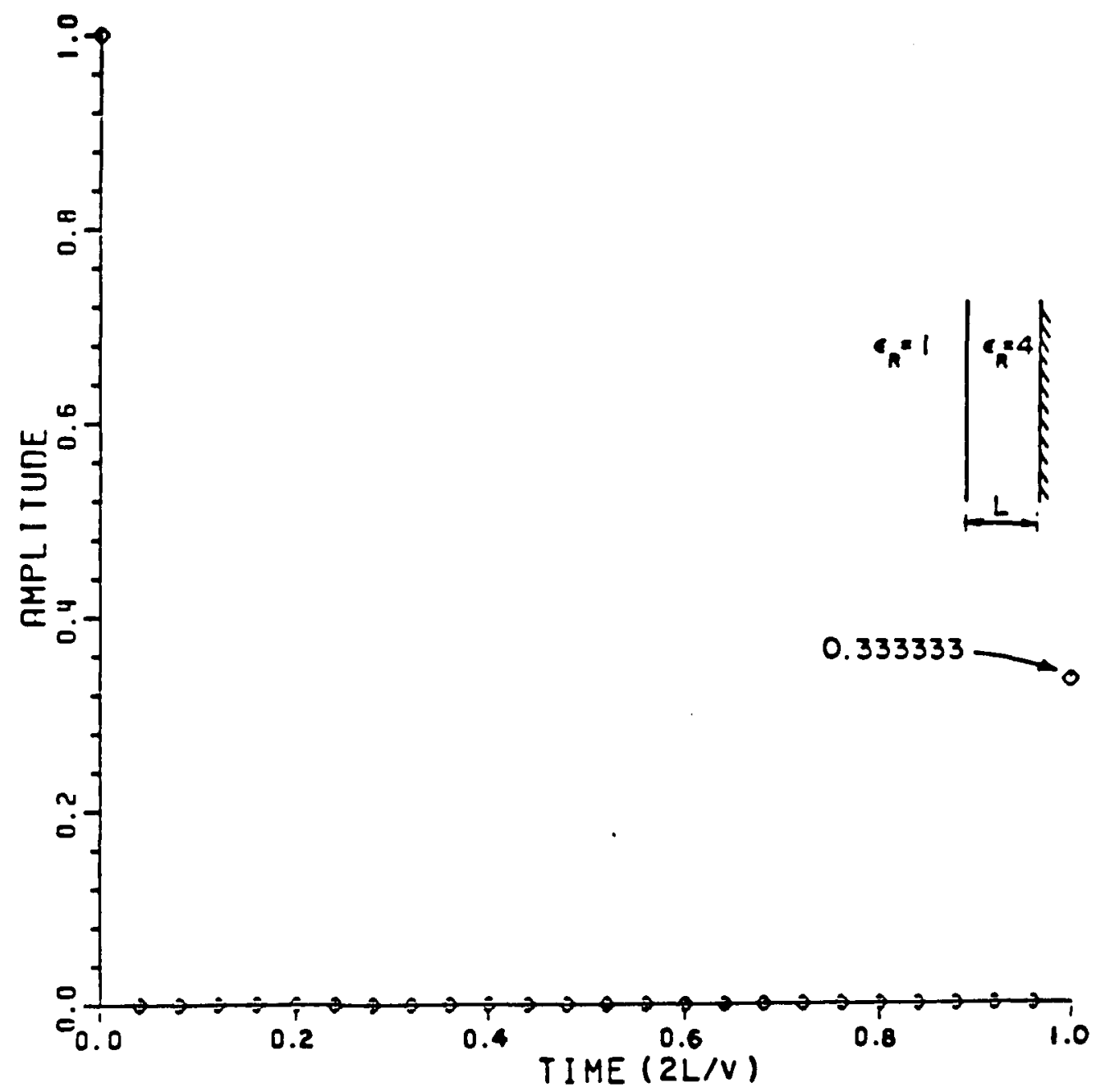

Figure 3.4. The K-pulse for normal incidence on the grounded dielectric slab resulted after optimization with the initial guess corresponding to Figure 3.3. (each diamond's height represents the weighting of an impulse). 


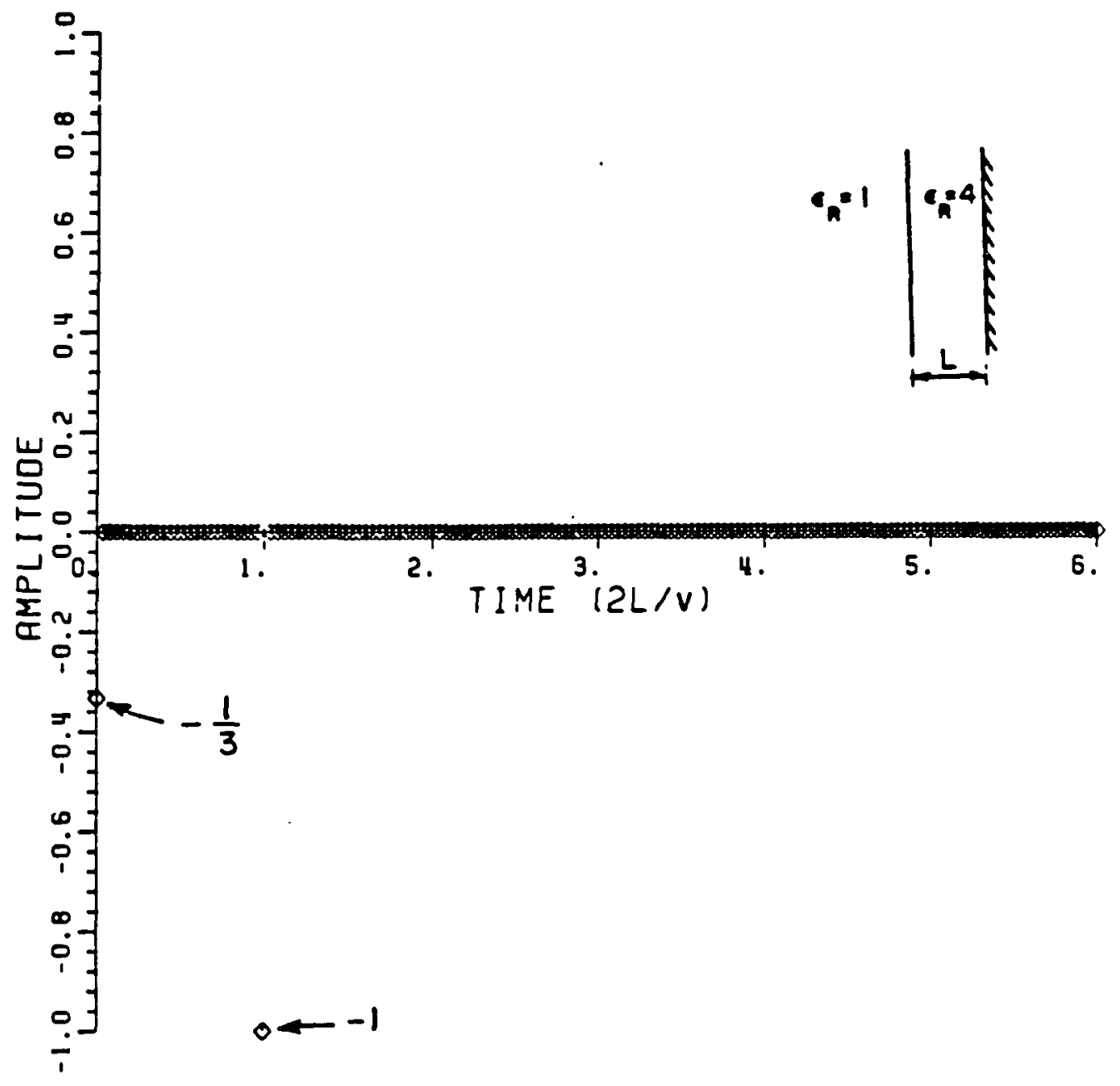

Figure 3.5. Response of the grounded dielectric slab to the K-pulse shown in Figure 3.4. (each diamond's height represents the weighting of an impulse). 

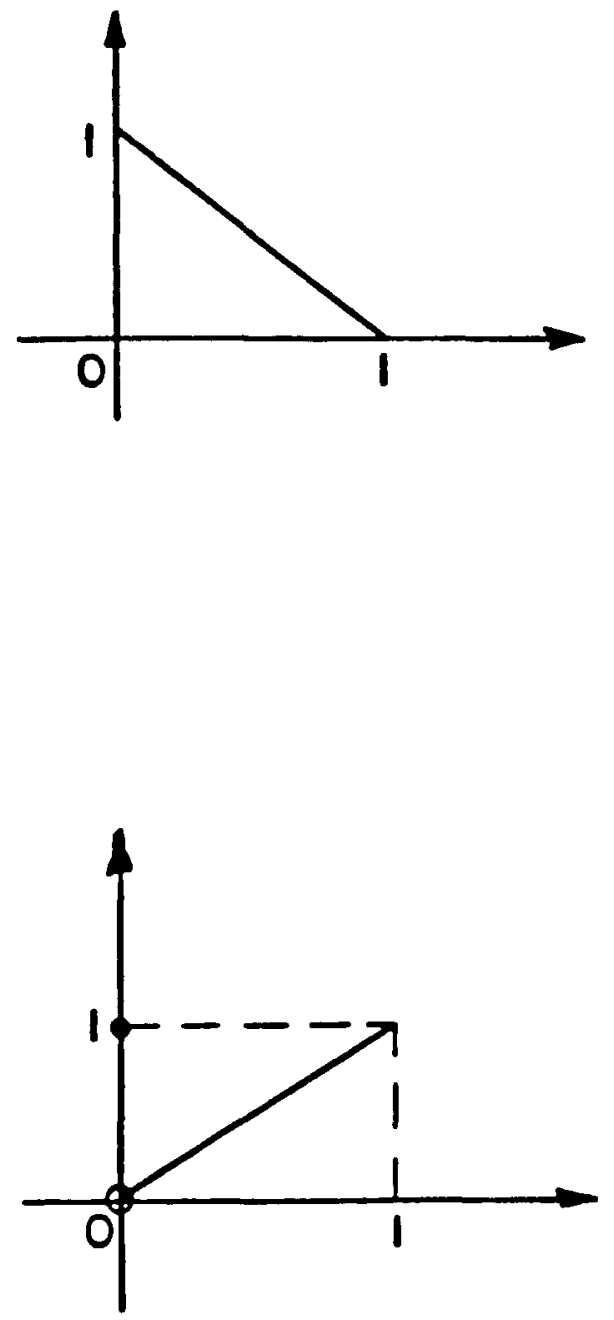

Figure 3.6. Two other initial guesses for the K-pulse which after optimization yield the same $K$-pulse as Figure 3.4 . 


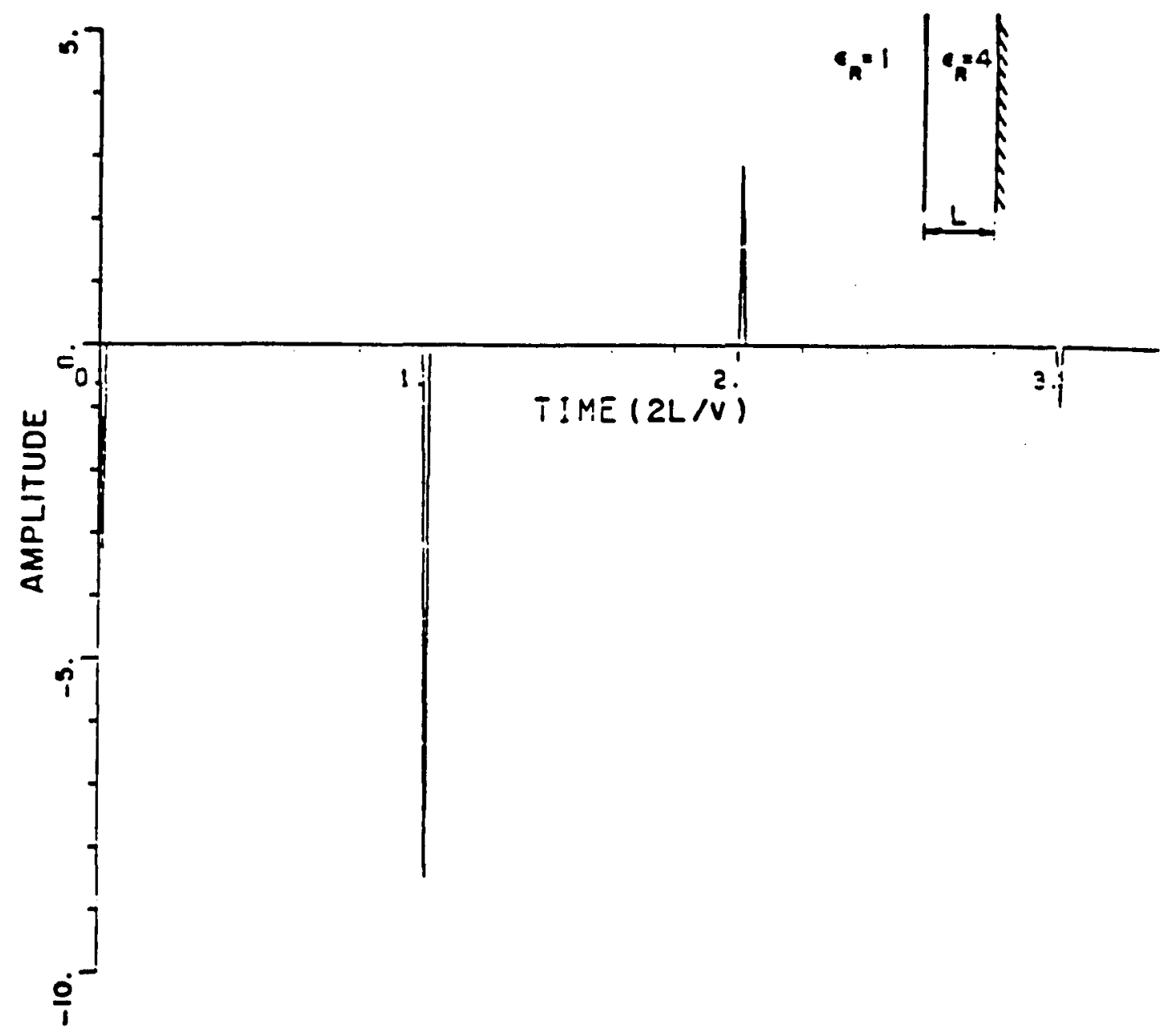

Figure 3.7. Impulse response (Fourier synthesized) of the grounded dielectric slab to a normally incident plane wave. $(k L=0.01 \pi(0.01 \pi) 100 \pi)$. 


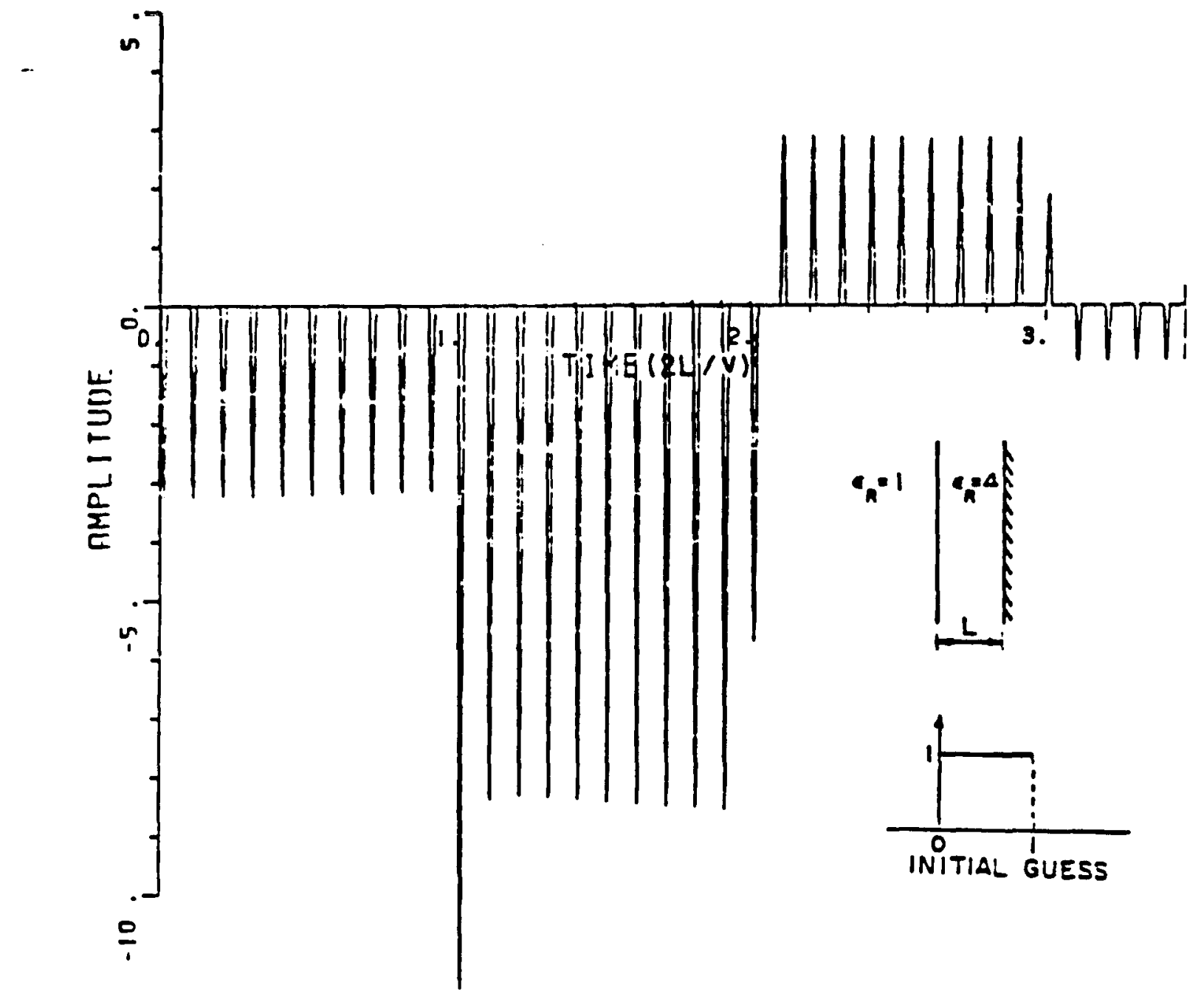

Figure 3.8. Response of the grounded dielectric slab to a set of unit impulses (11) which represents the first guess for the K-pulse. 


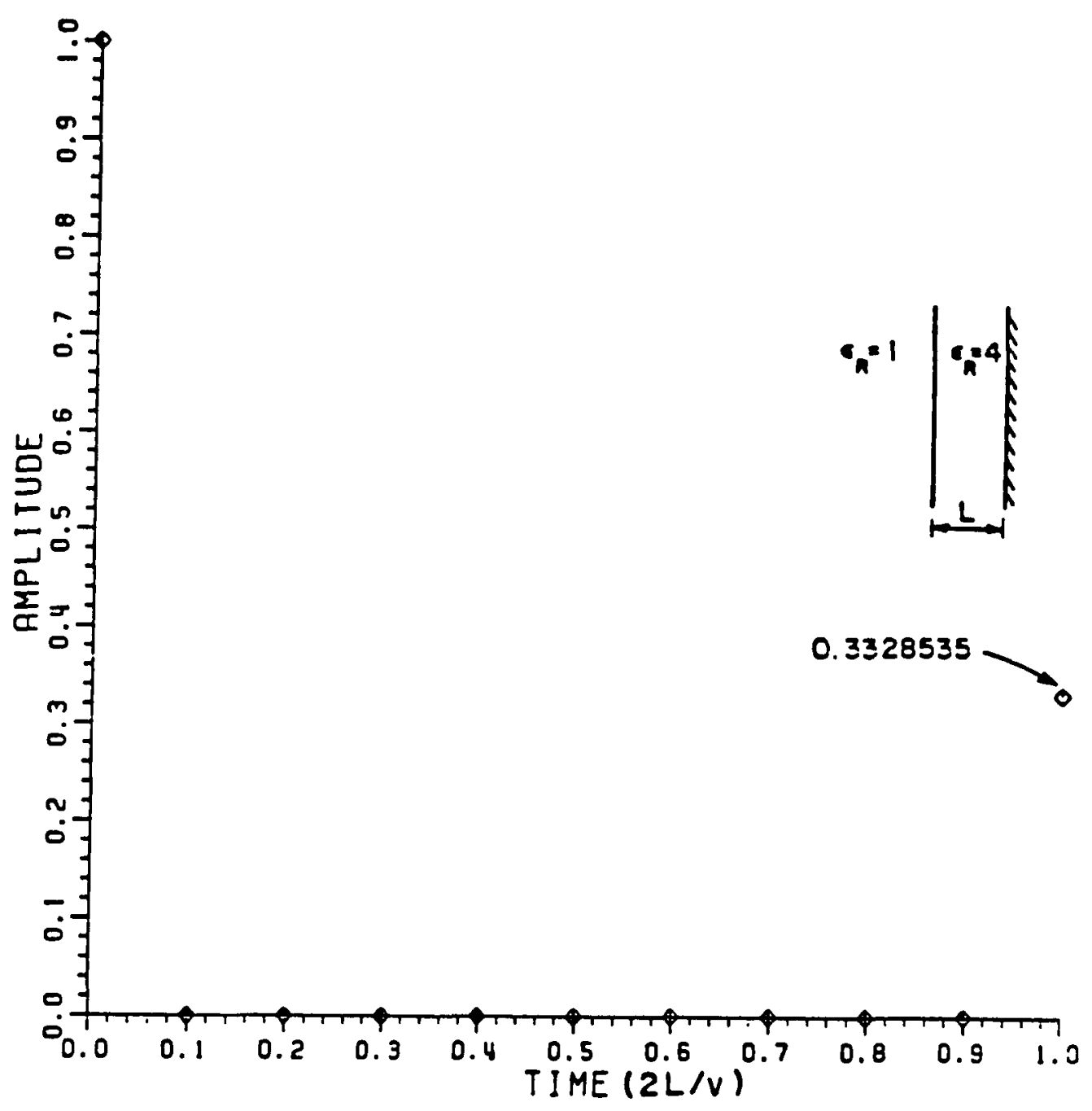

Figure 3.9. The K-pulse for normal incidence on the grounded dielectric slab resulted after optimization with the initial guess corresponding to Figure 3.8. (each diamond's height represents the weight of an impulse). 


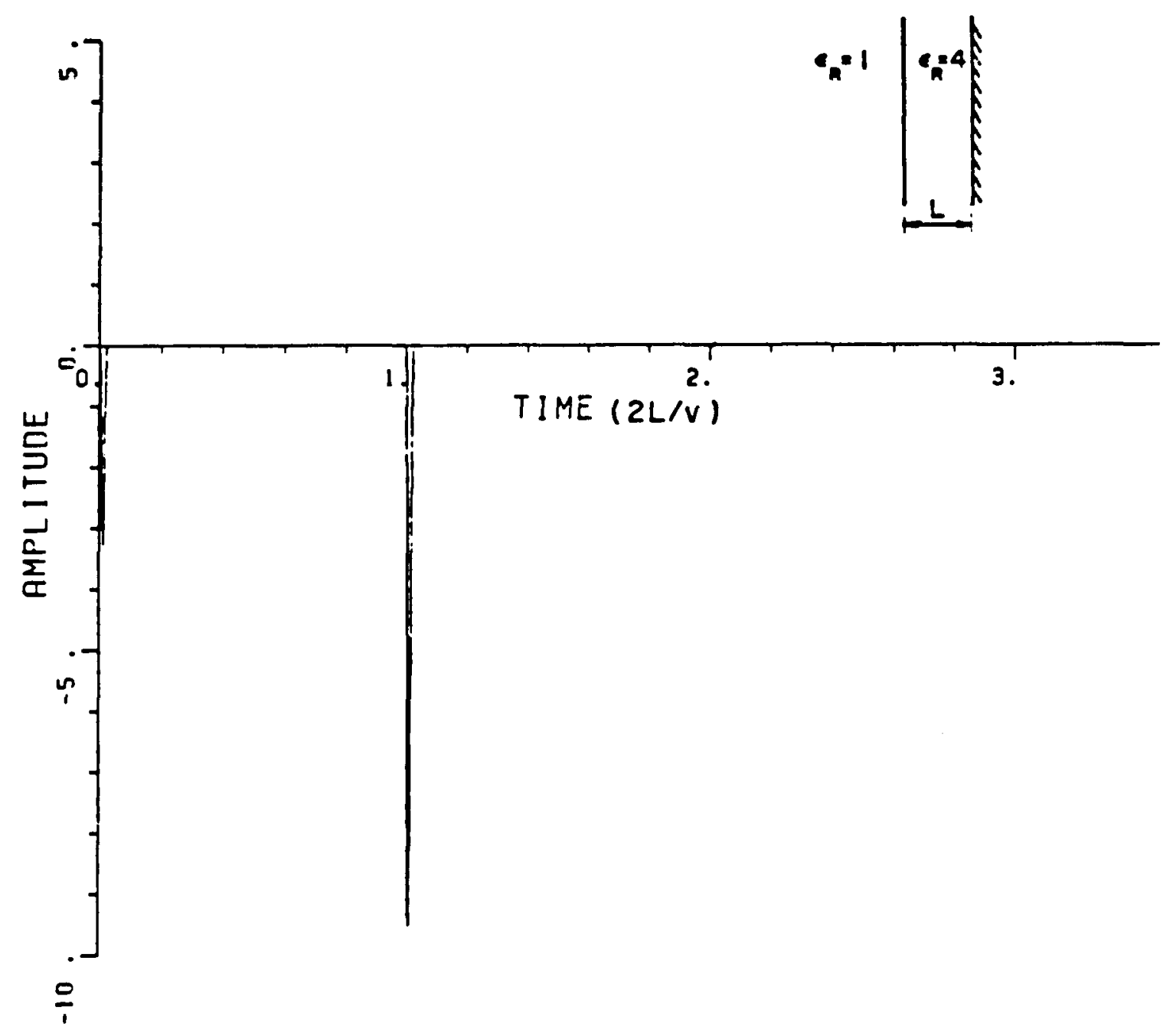

Figure 3.10. Response of the grounded dielectric slab to the normal incident of the K-pulse shown in Figure 3.9 . 


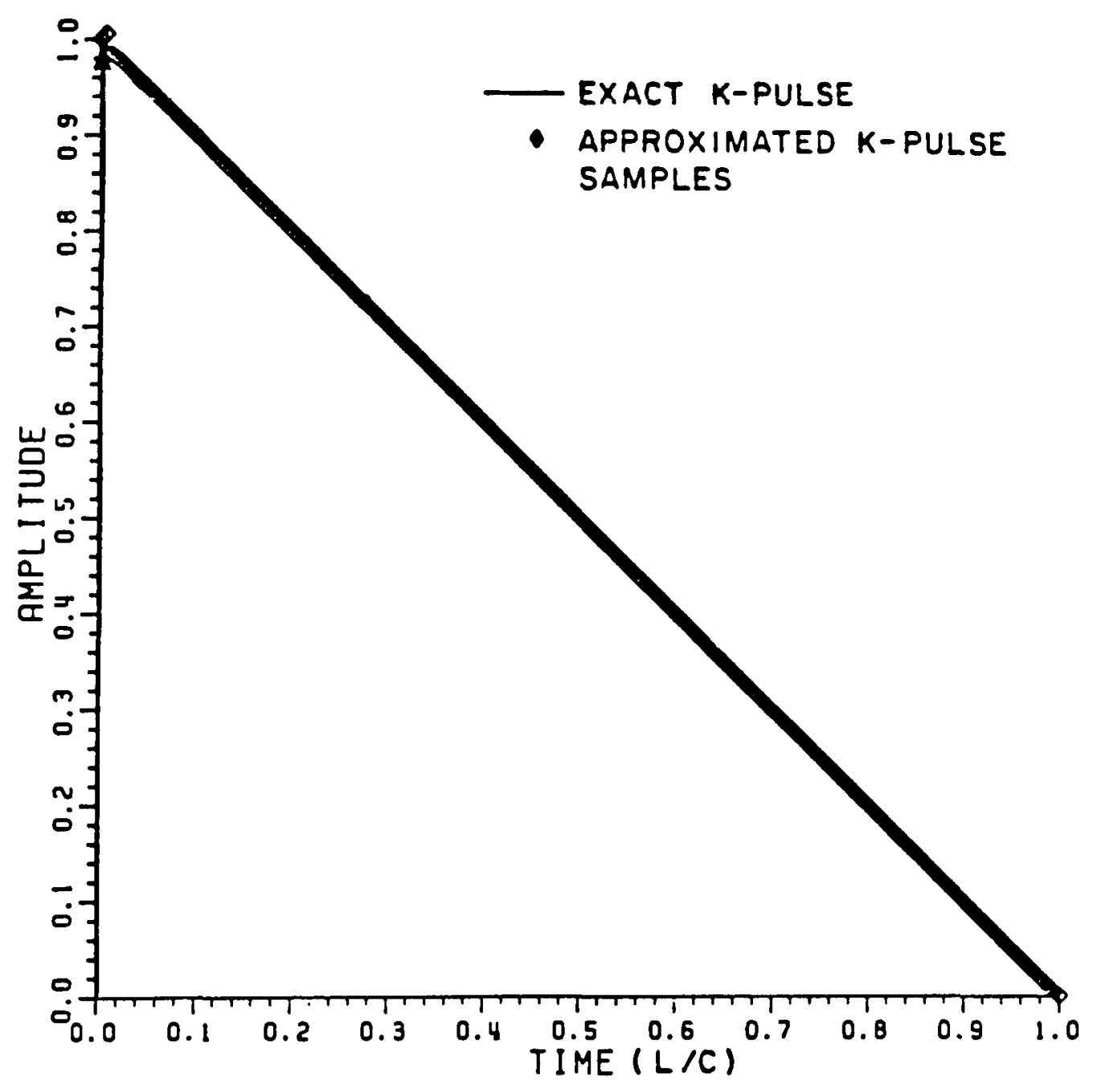

Figure 3.11. The exact and approximate $K$-pulse for the simulated model given in Equation (3.2). 


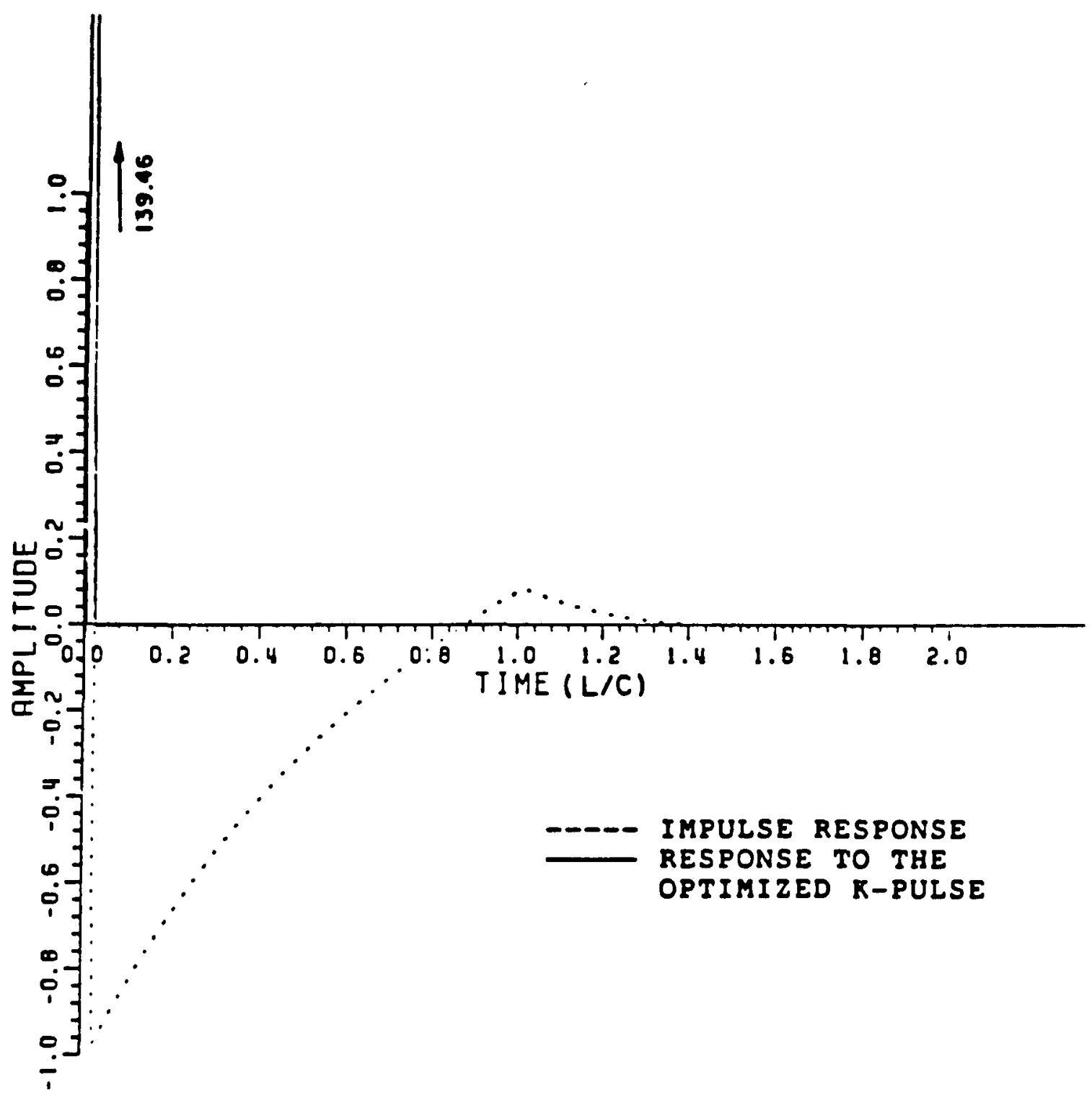

Figure 3.12. Impulse response and response to the approximate $K$-pulse shown in Figure 3.11 for the simulated model in Equation (3.2). Both responses have an impulsive singularity at the start of the waveform. $(K L=0.2 \pi(0.2 \pi) 400 \pi)$. 


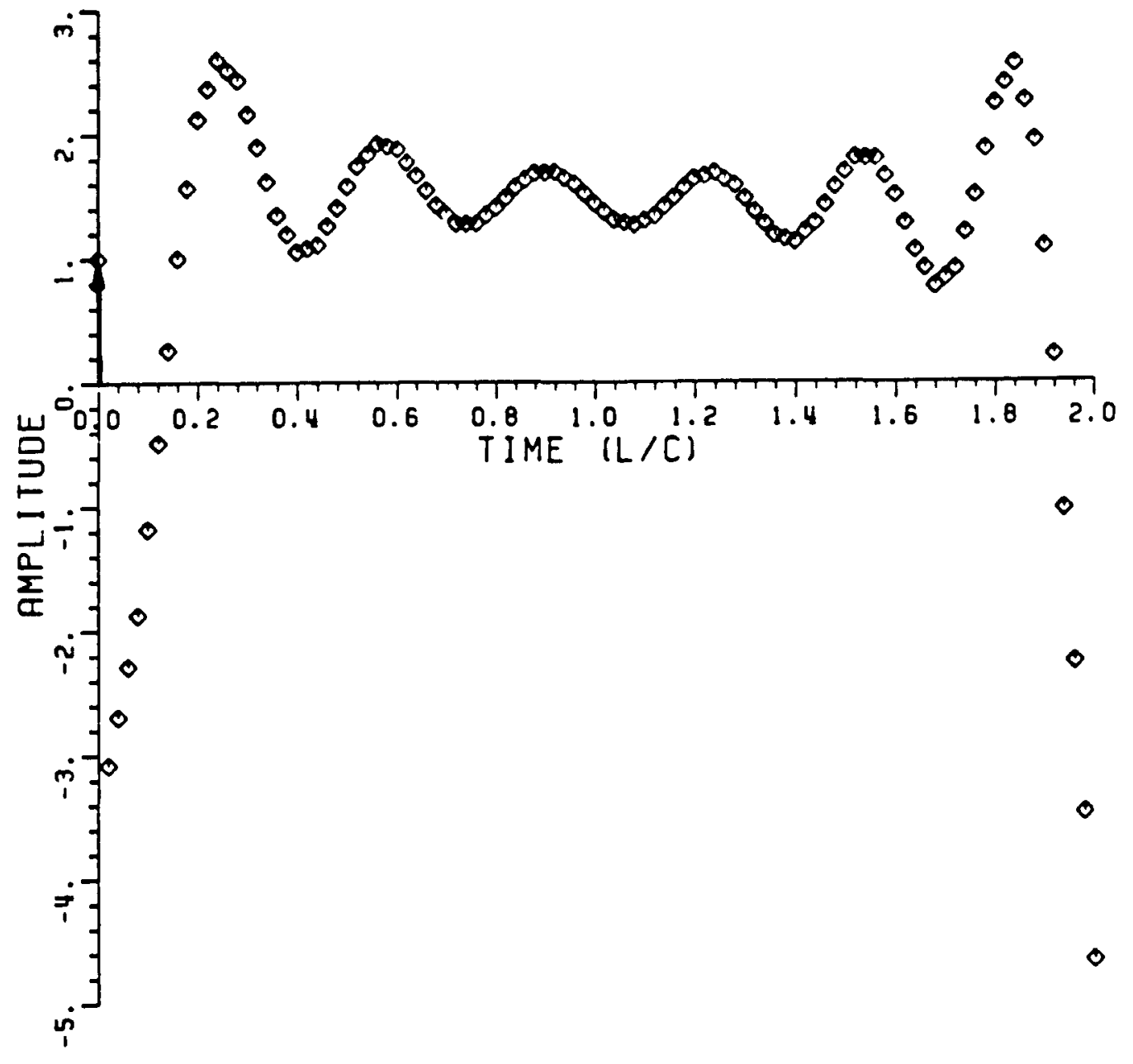

Figure 3.13. K-pulse obtained using the Fourier synthesized impulse response of the conducting straight wire at $\phi=30^{\circ}$ with $K L=0.08 \pi(0.08 \pi) 8 \pi$. 


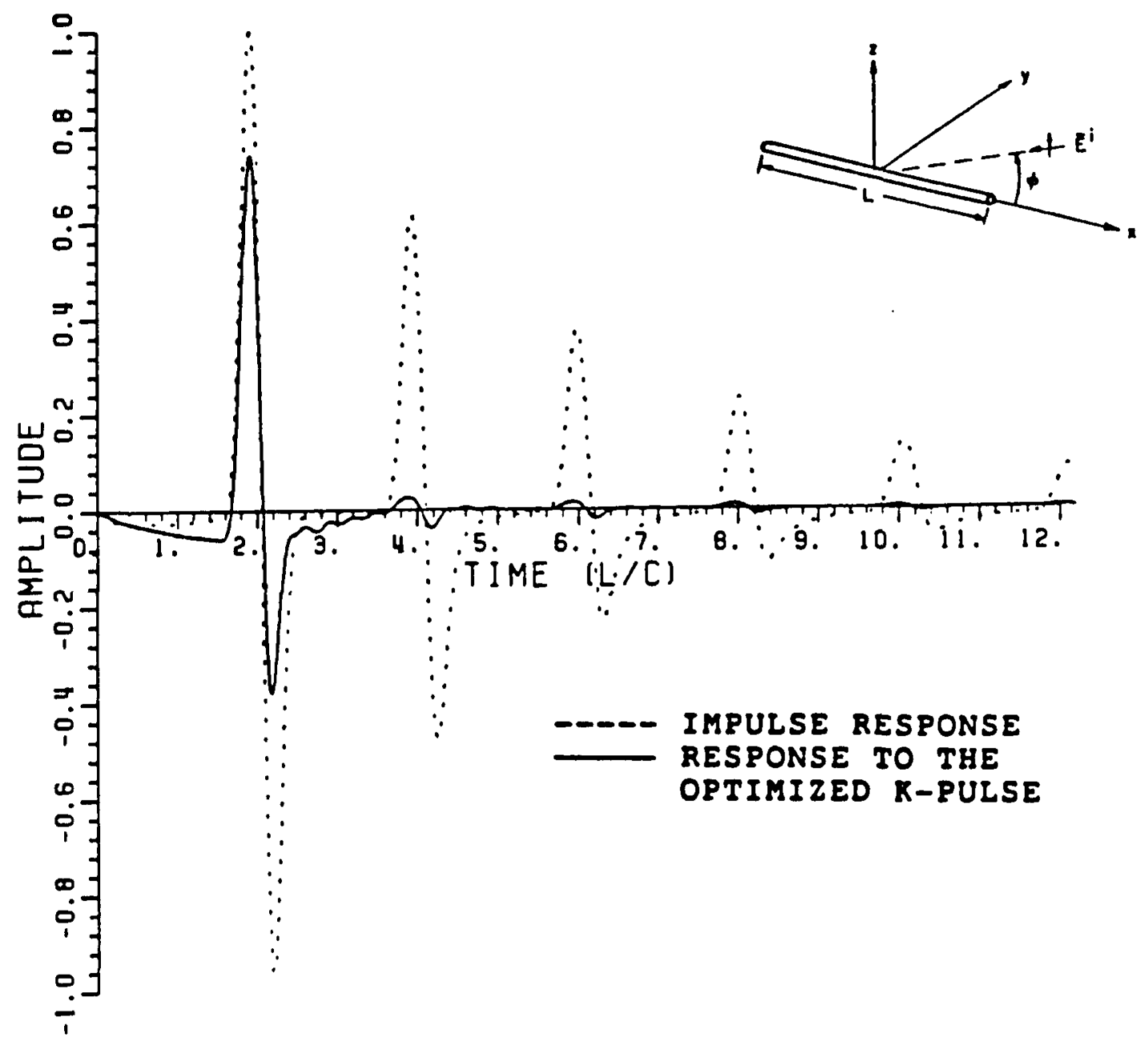

Figure 3.14. Impulse response and response to the K-pulse shown in Figure 3.13 for the straight wire at $\phi=30^{\circ}$ with $K L=0.08 \pi(0.08 \pi) 8 \pi$. 


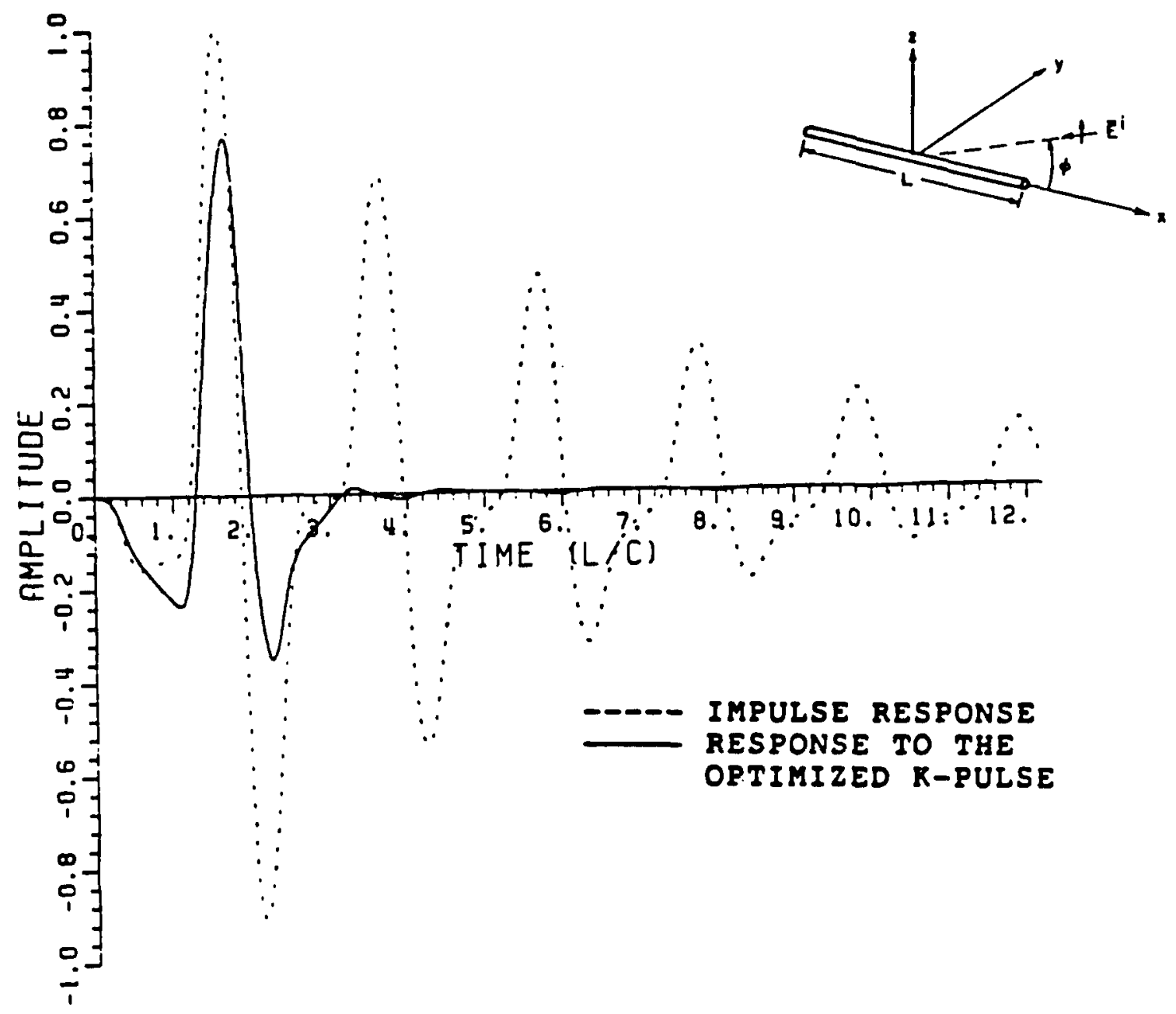

Figure 3.15. Impulse response and response to the $K$-pulse shown in Figure 3.13 for the straight wire at $\phi=60^{\circ}$ with $K L=0.08 \pi(0.08 \pi) 8 \pi$. 


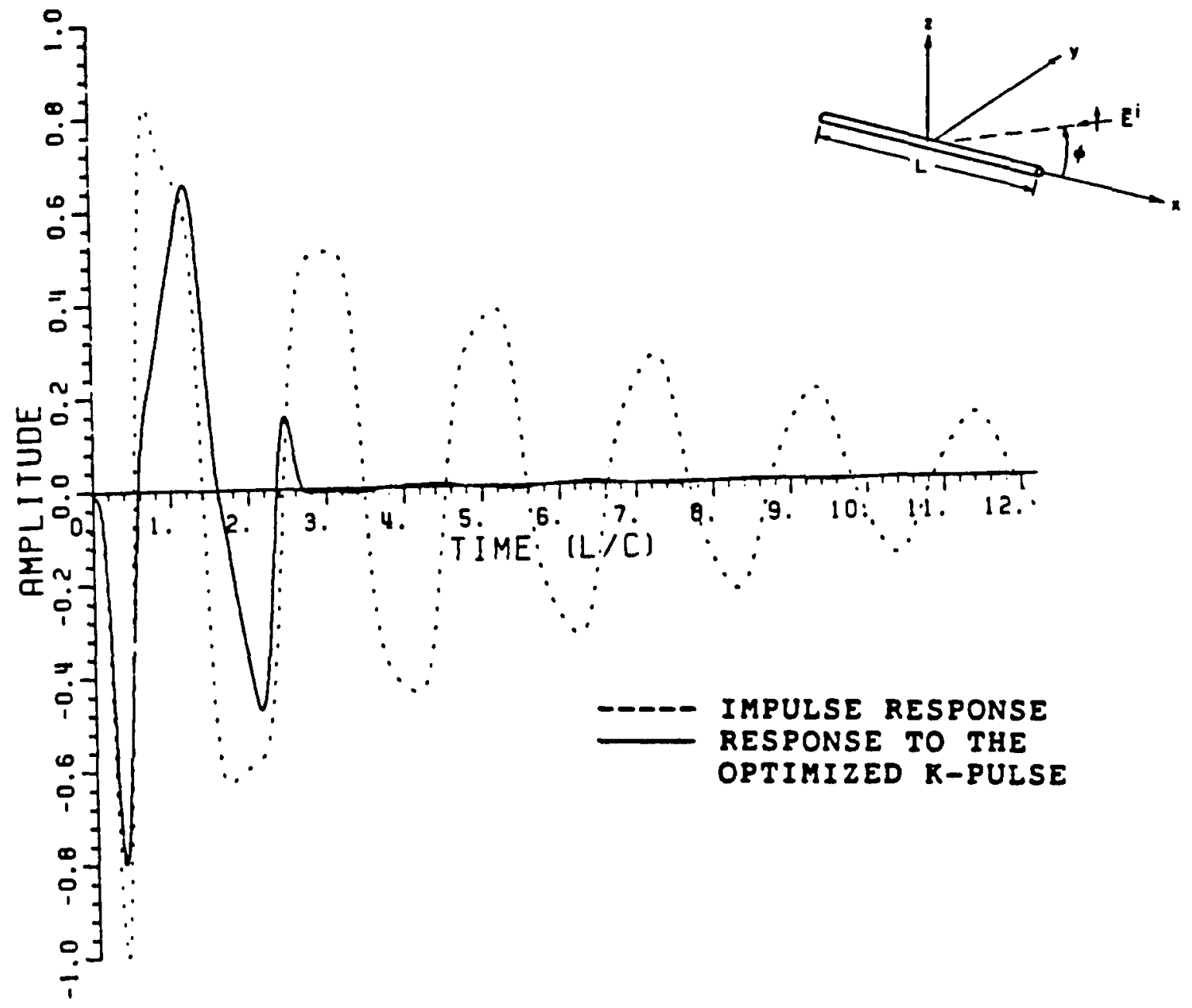

Figure 3.16. Impulse response and response to the $K$-pulse shown in Figure 3.13 for the straight wire at $\phi=90^{\circ}$ with $k L=0.08 \pi(0.08 \pi) 8 \pi$. 


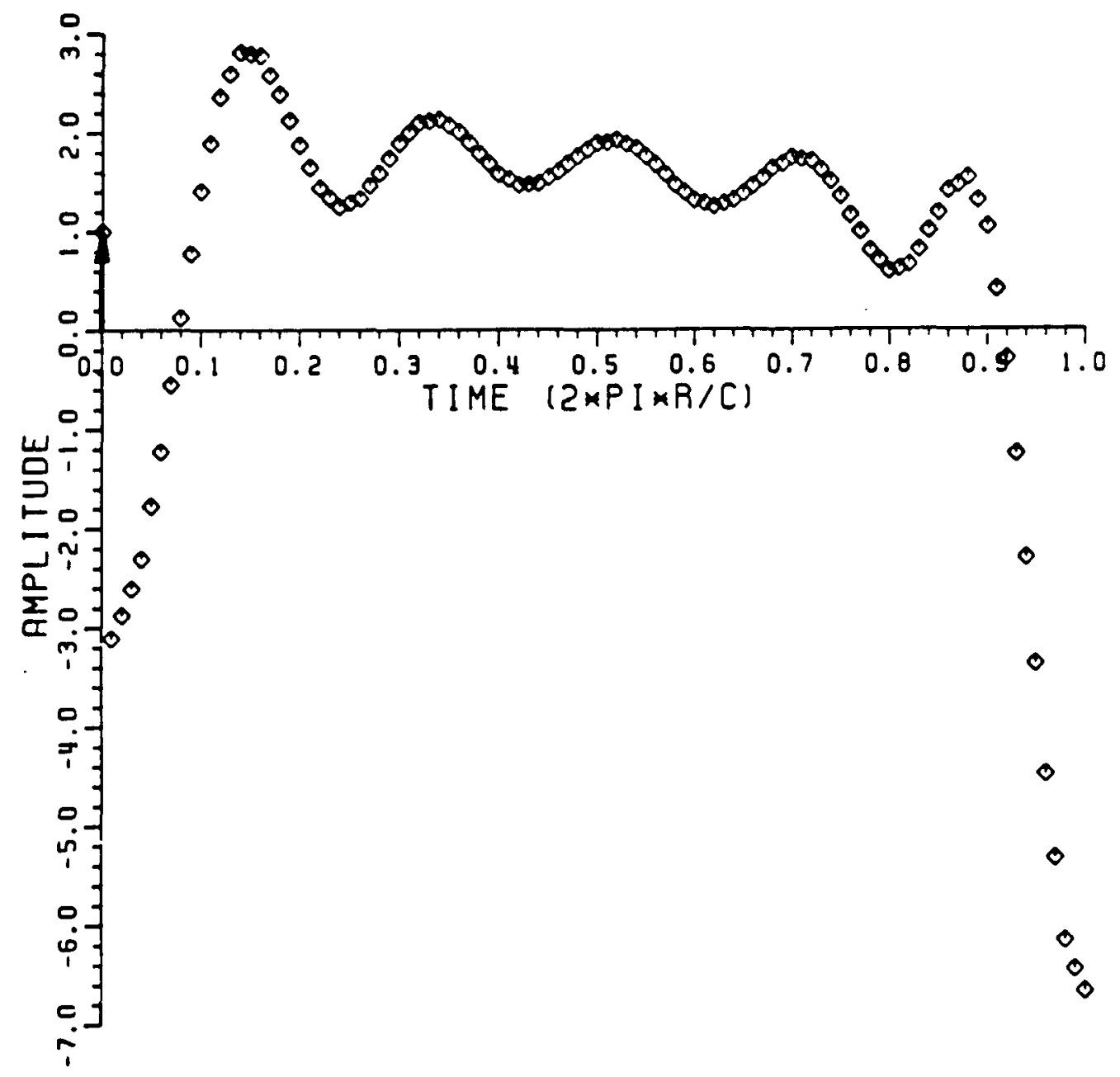

Figure 3.17. K-pulse obtained using the Fourier synthesized impulse response of the conducting circular loop at $\theta=45^{\circ}$, $\hat{\theta}$-polarization with $k r=0.02 \pi(0.02 \pi) 4 \pi$. 


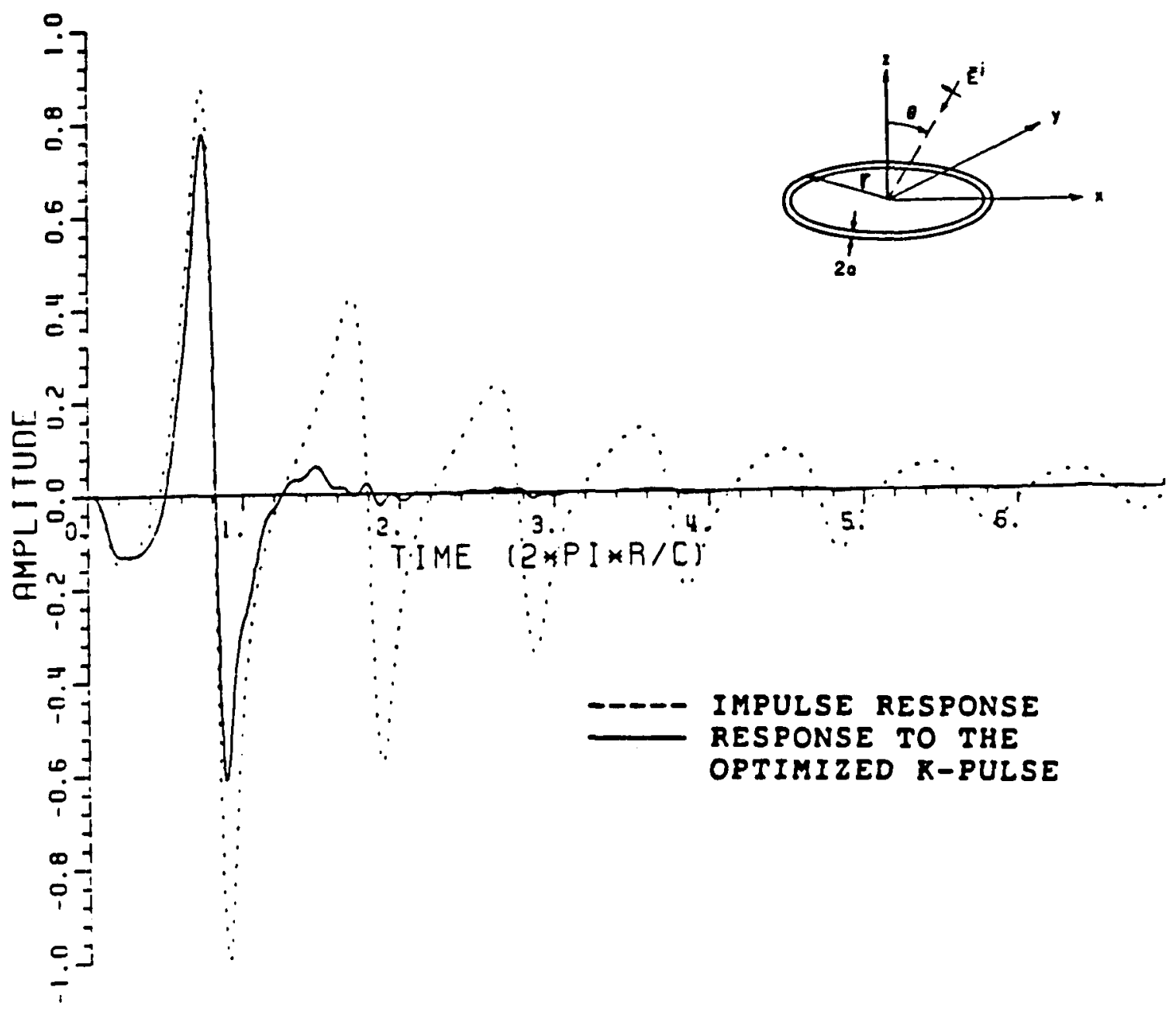

Figure 3.18. Impulse response and response to the $K$-pulse shown in Figure 3.17 for the conducting circular loop at $\theta=45^{\circ}$, $\hat{\theta}$-polarization with $\mathrm{kr}=0.02 \pi(0.02 \pi) 4 \pi$. 


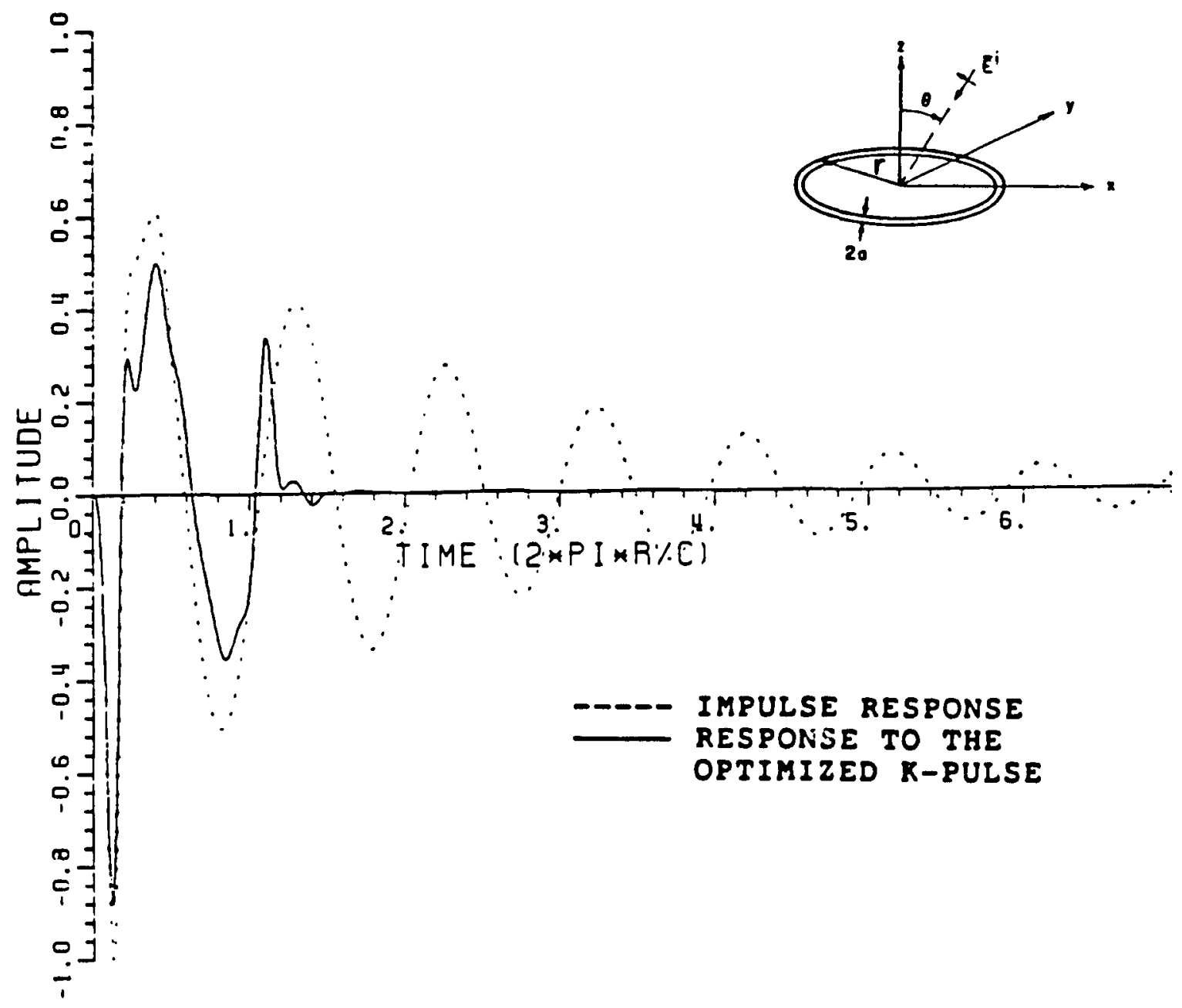

Figure 3.19. Impulse response and response to the $K$-pulse shown in Figure 3.17 for the conducting circular loop at $\theta=0^{\circ}$, $\phi$-polarization with $k r=0.02 \pi(0.02 \pi) 4 \pi$. 


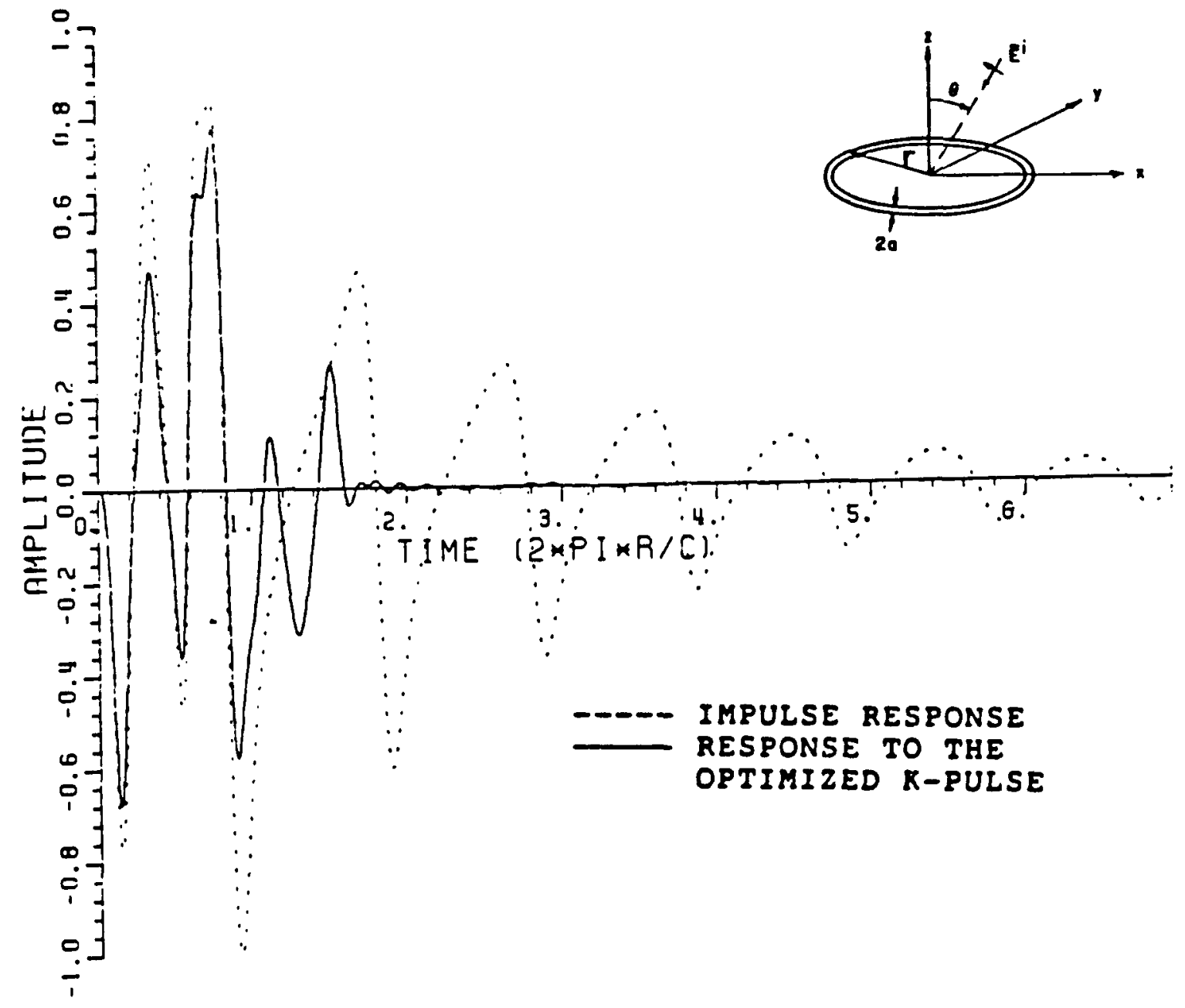

Figure 3.20. Impulse response and response to the K-pulse shown in Figure 3.17 for the conducting circular loop at $\theta=45^{\circ}$, $\hat{\phi}$-polarization with $\mathrm{kr}=0.02 \pi(0.02 \pi) 4 \pi$. 


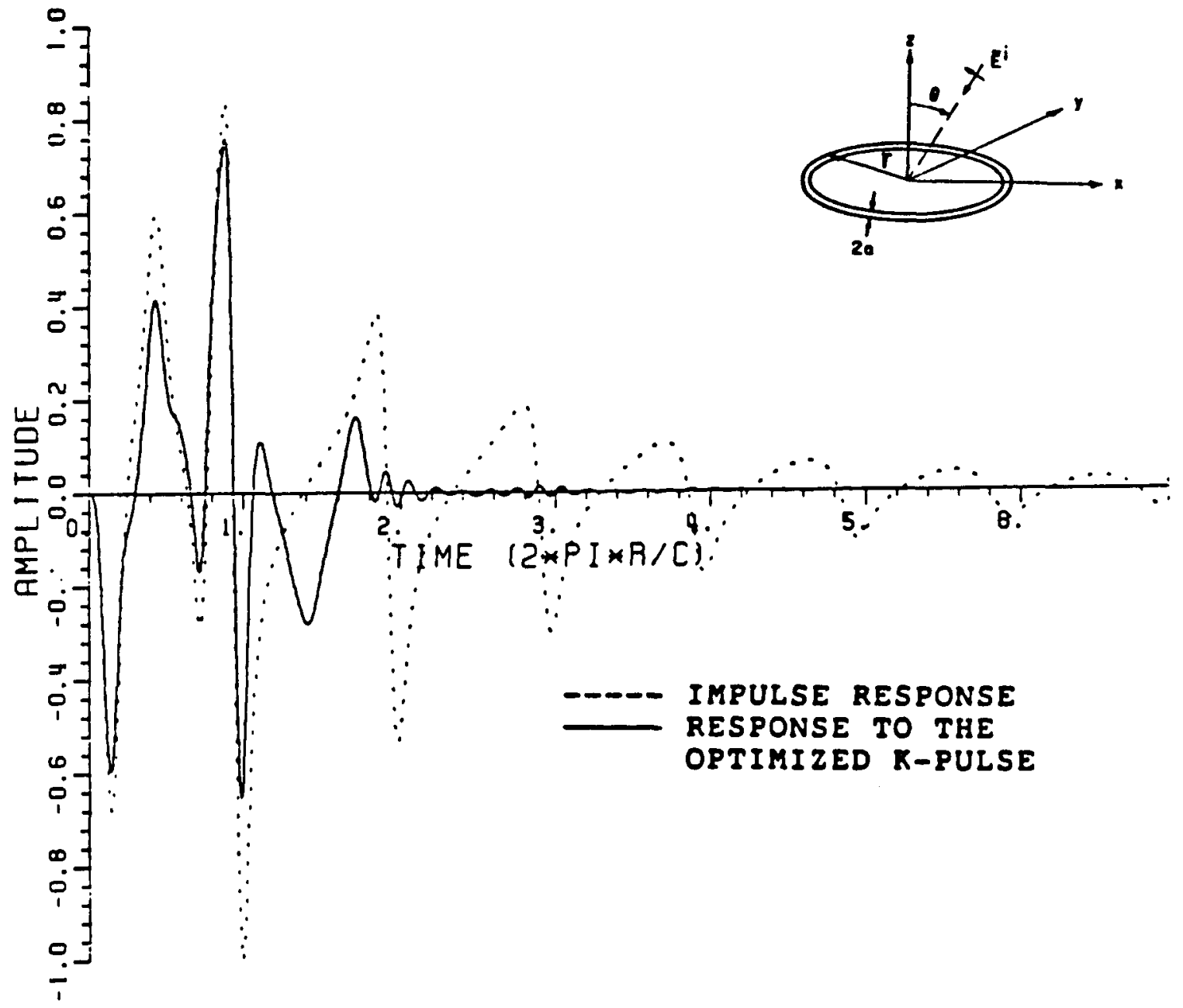

Figure 3.21. Impulse response and response to the $K$-pulse shown in Figure 3.17 for the conducting circular loop at $\theta=90^{\circ}$, $\hat{\phi}$-polarization with $k r=0.02 \pi(0.02 \pi) 4 \pi$. 


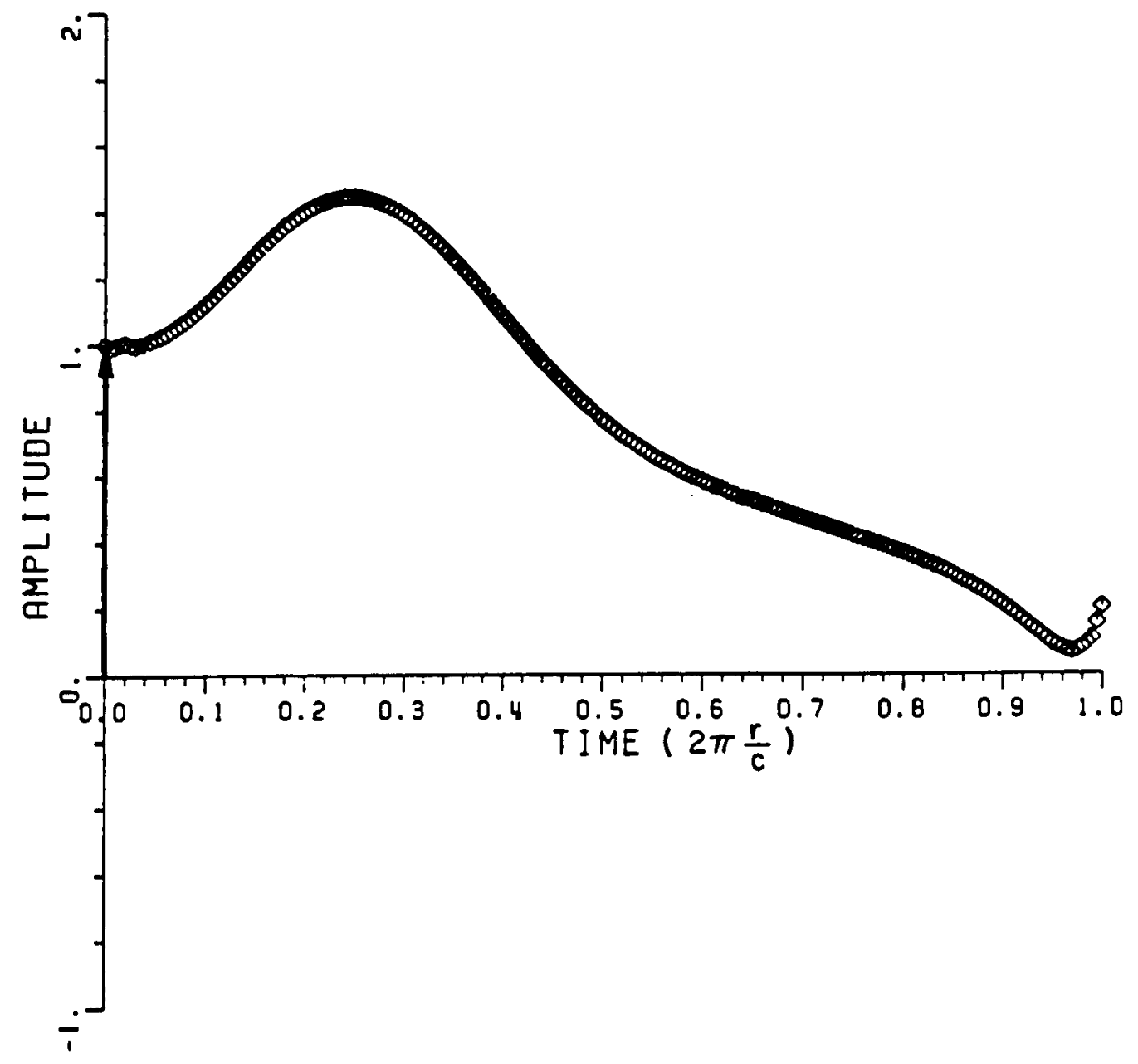

Figure 3.22. K-pulse obtained using the Fourier synthesized impulse response of the conducting sphere in the backscattered direction with $\mathrm{kr}=0.1(0.1) 66$. 


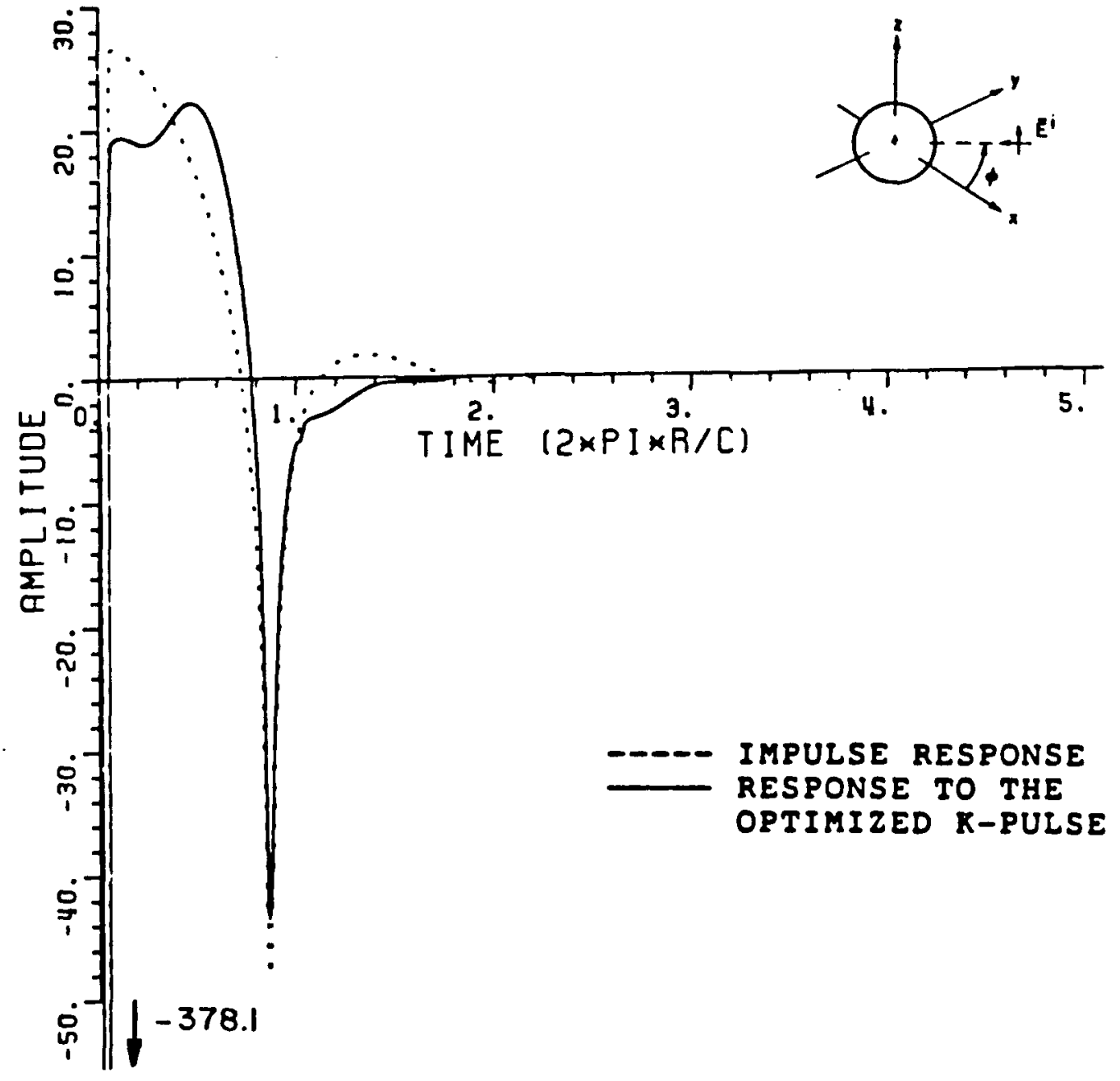

(a) No expansion of the amplitude scale.

Figure 3.23. Impulse response and response to the K-pulse shown in Figure 3.22 for the conducting sphere in the backscattered direction with $\mathrm{kr}=0.1(0.1) 66$. 


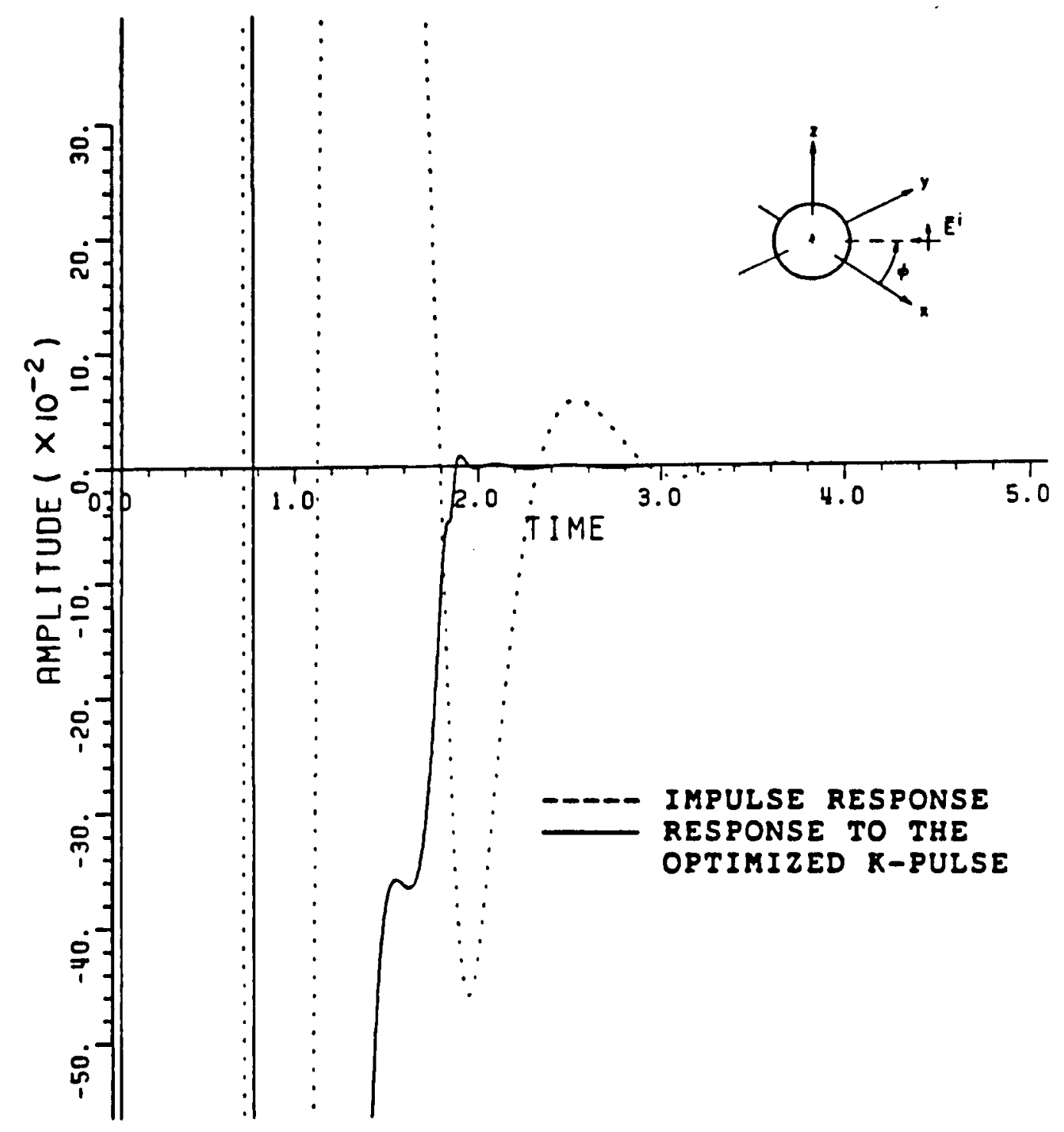

(b) The amplitude scale is expanded by 100 .

Figure 3.23. Continued. 


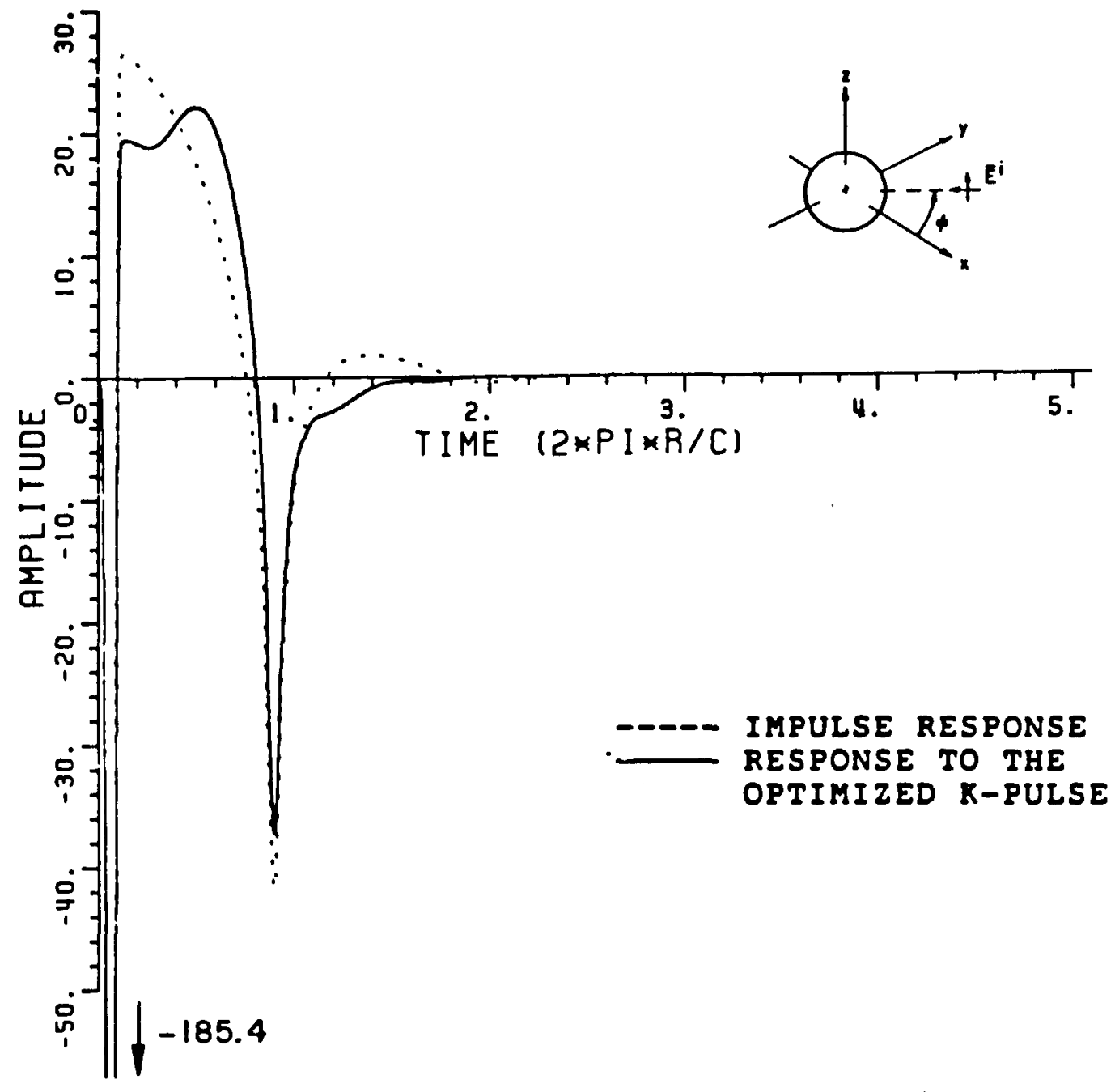

(a) No expansion of the amplitude scale.

Figure 3.24. Impulse response and response to the $K$-pulse shown in Figure 3.22 for the conducting sphere in the backscattered direction with $\mathrm{kr}=0.1(0.1) 33.5$. 


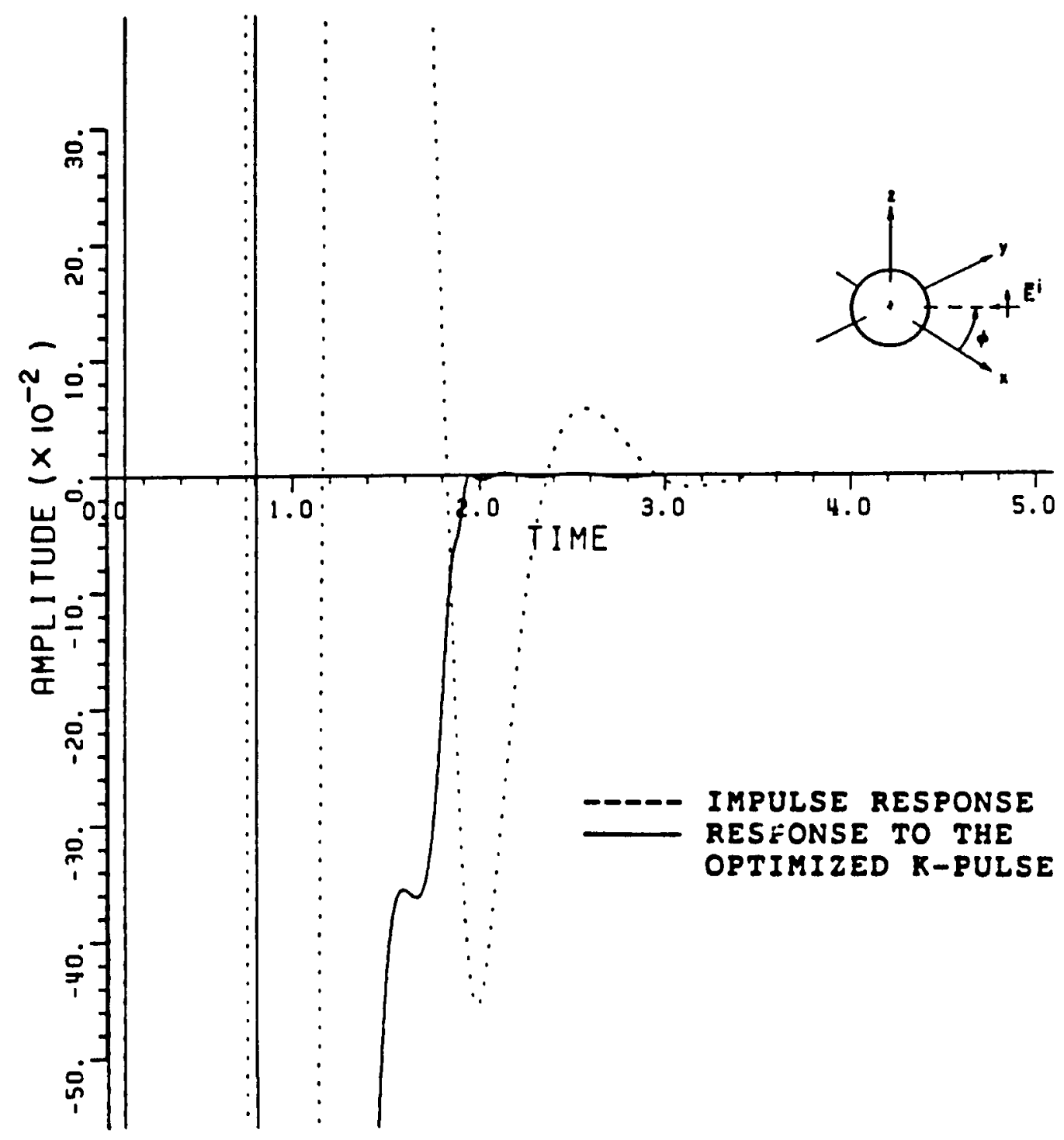

(b) The amplitude scale is expanded by 100 .

Figure 3.24. Continued. 


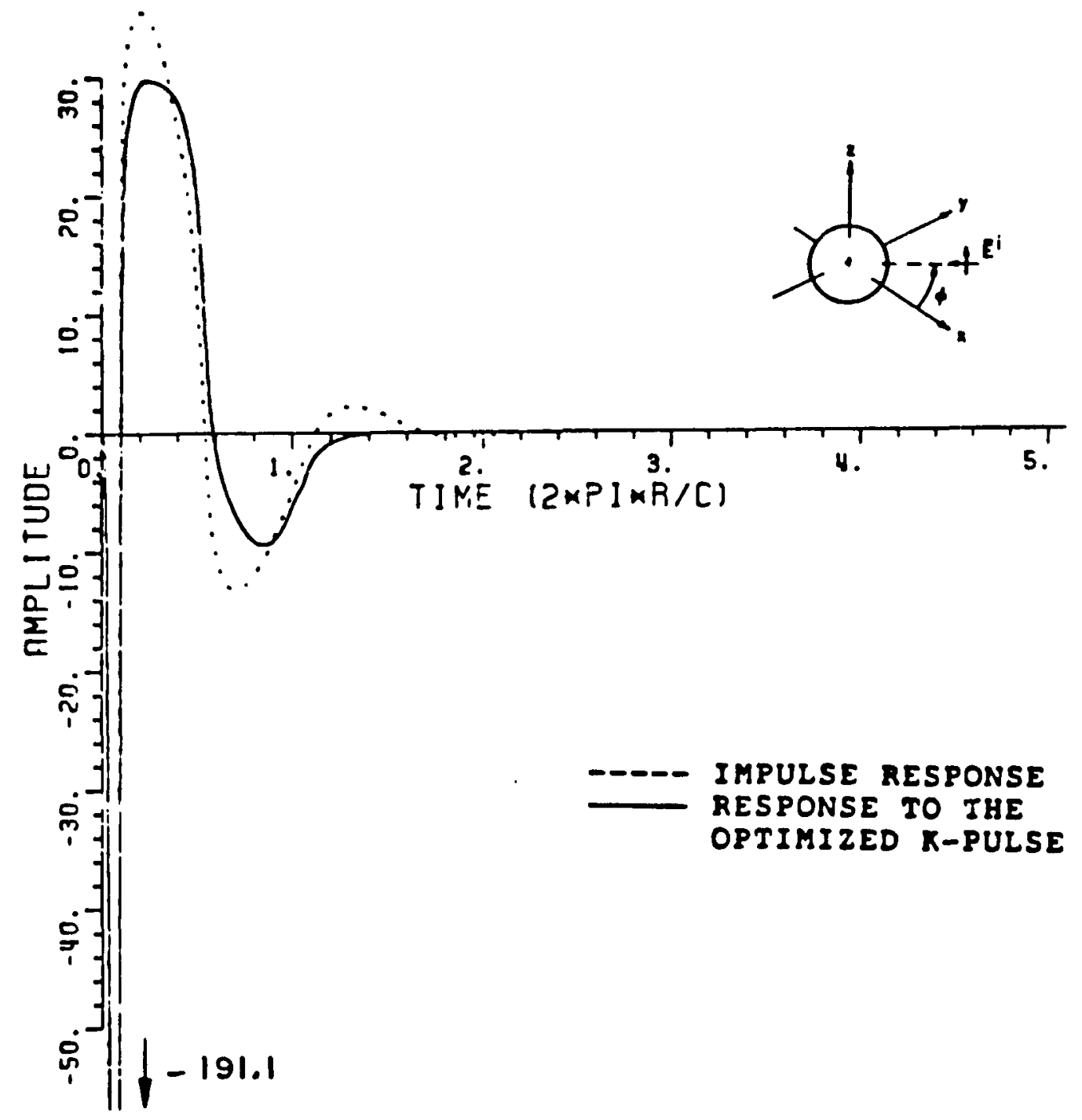

(a) No expansion of the amplitude scale.

Figure 3.25. Impulse response and response to the $K$-pulse shown in Figure 3.22 for the conducting sphere at a bistatic angle of $\phi=90^{\circ}$, vertical polarization with $k r=0.1(0.1) 33.5$. 


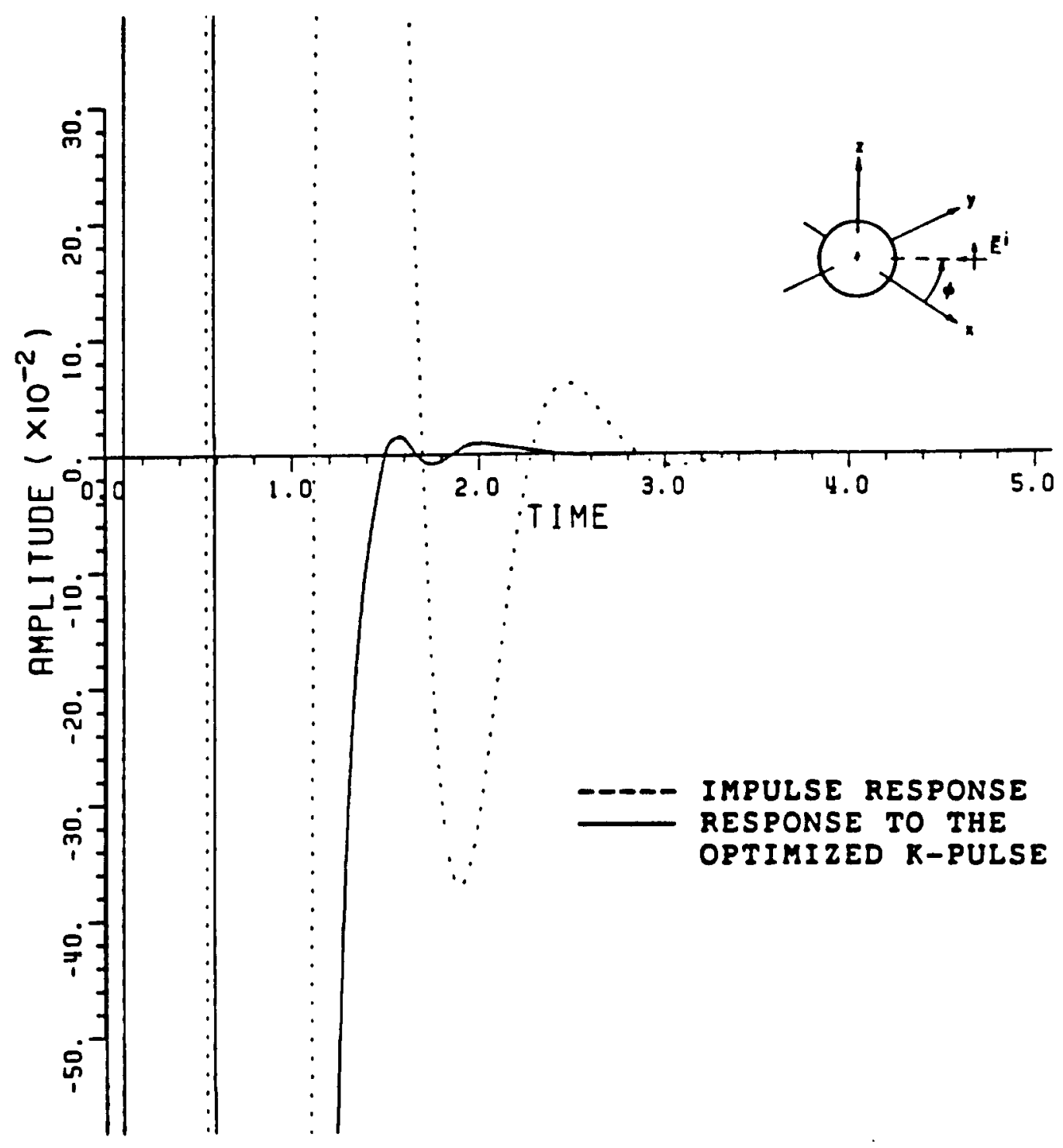

(b) The amplitude scale is expanded by 100 .

Figure 3.25. Continued. 


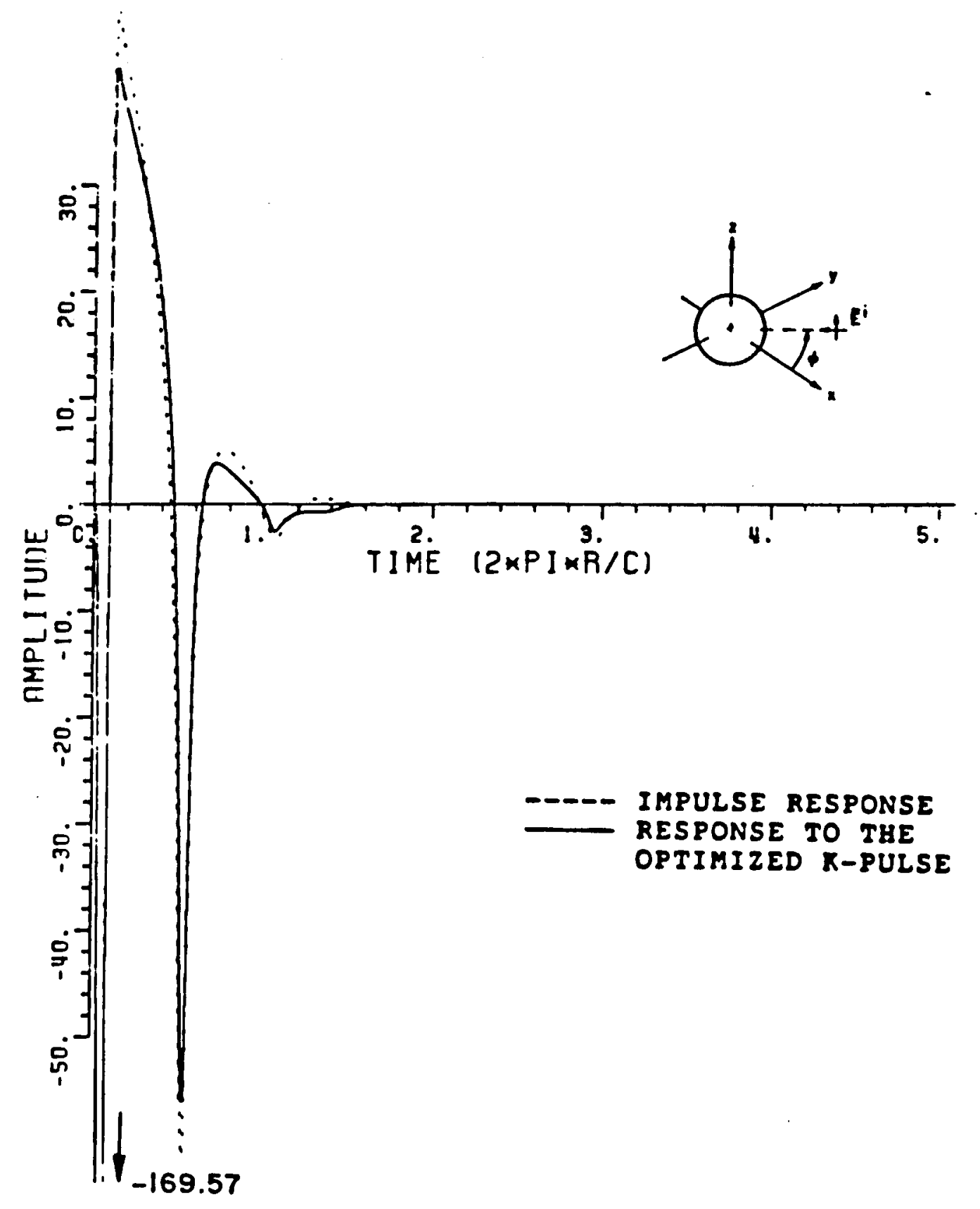

(a) No expansion of the amplitude scale.

Figure 3.26. Impulse response and response to the $K$-pulse shown in Figure 3.22 for the conducting sphere at a bistatic angle of $\phi=90^{\circ}$, horizontal polarization with $k r=0.1(0.1) 33.5$. 


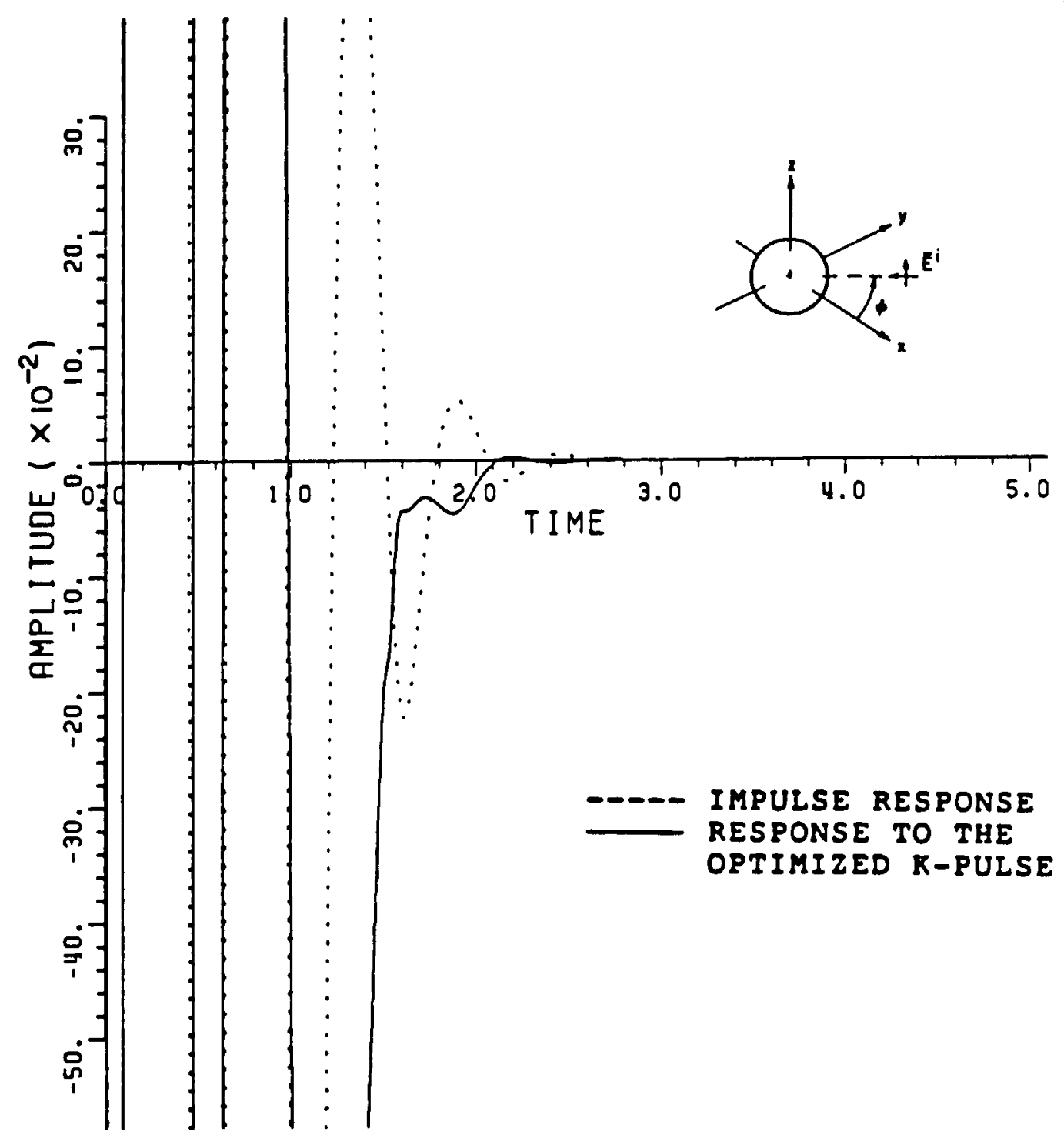

(b) The amplitude scale is expanded by 100 .

Fiogure 3.26. Continued. 


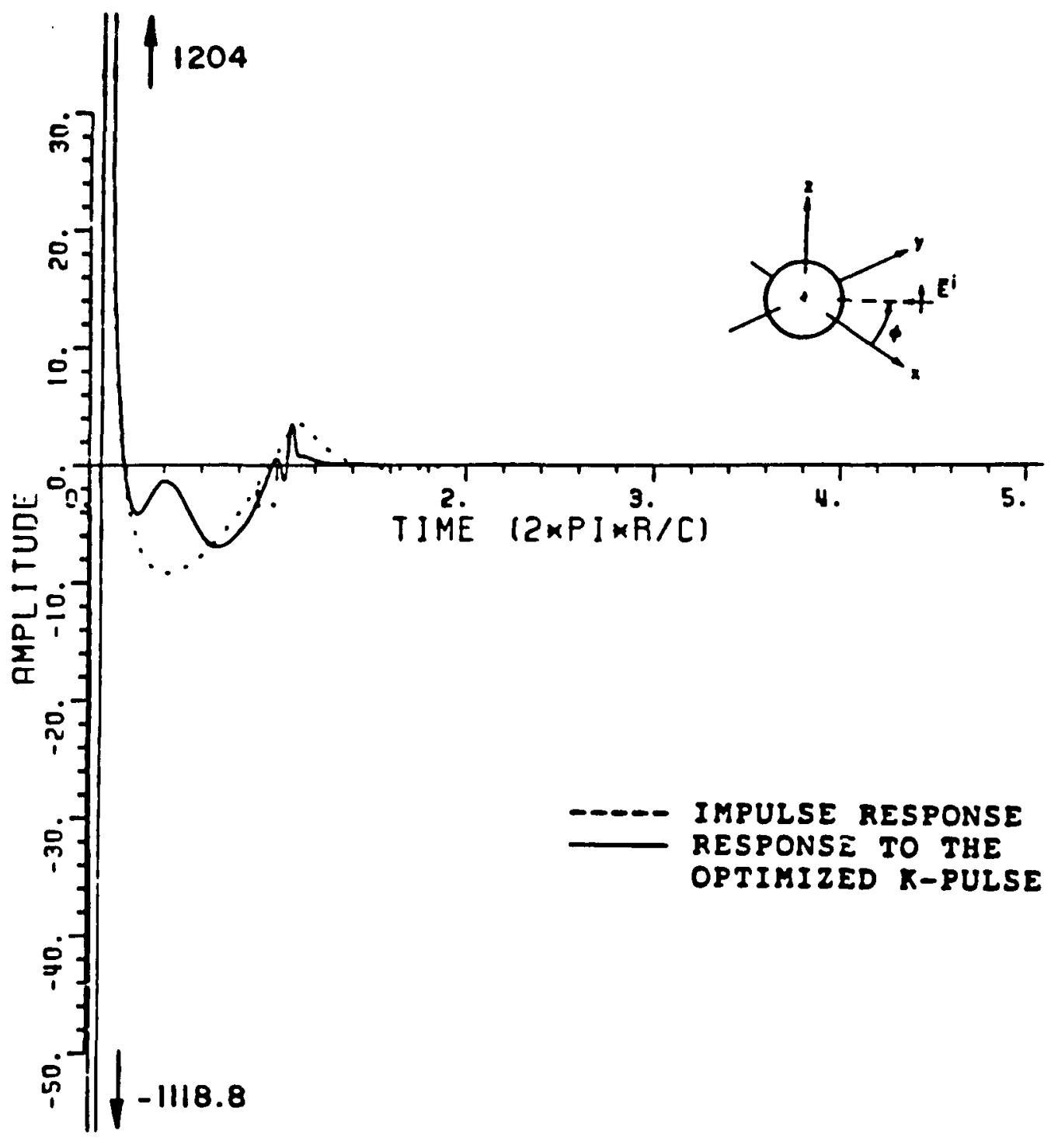

(a) No expansion of the amplitude scale.

Figure 3.27. Impulse response and response to the $K$-pulse shown in Figure 3.22 for the conducting sphere at a bistatic angle of $\phi=180^{\circ}$, vertical polarization with $k r=0.1(0.1) 33.5$. 


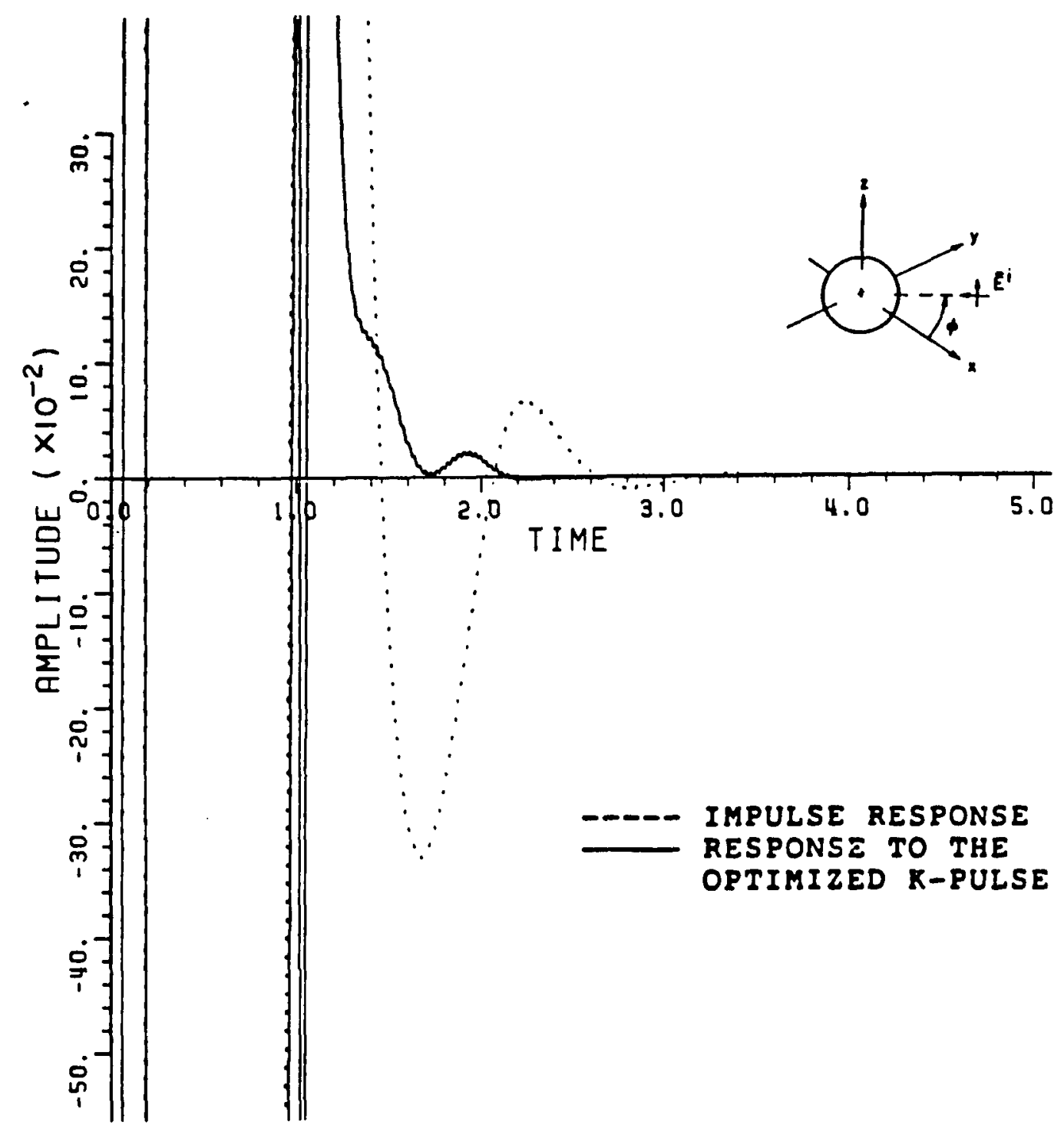

(b) The amplitude scale is expanded by 100 .

Figure 3.27. Continued. 


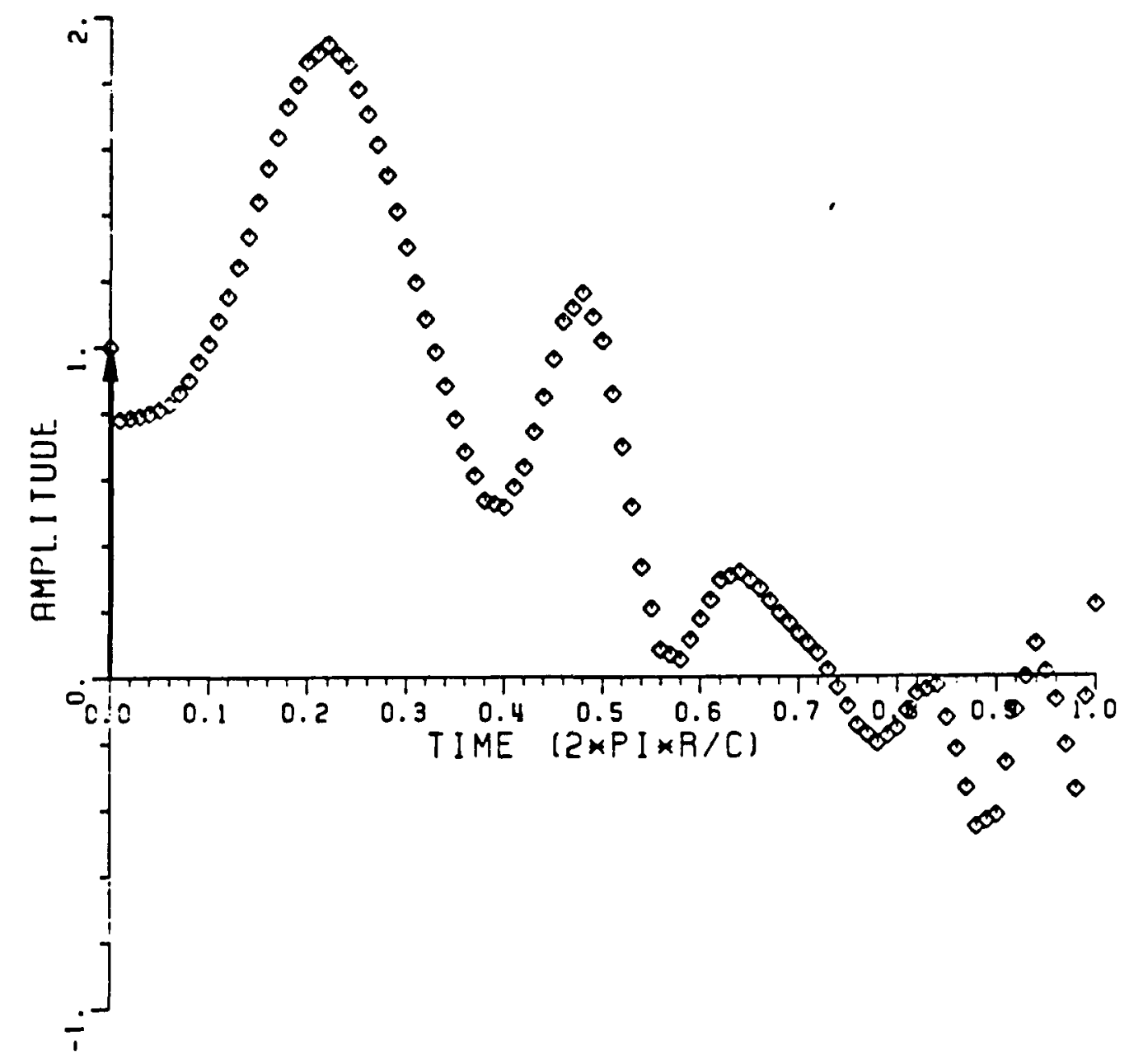

Figure 3.28. K-pulse obtained using the Fourier synthesized impulse response of the conducting circular disk at $\theta=45^{\circ}$, $\hat{\theta}$-polarization with $\mathrm{kr}=0.16(0.16) 16$. 


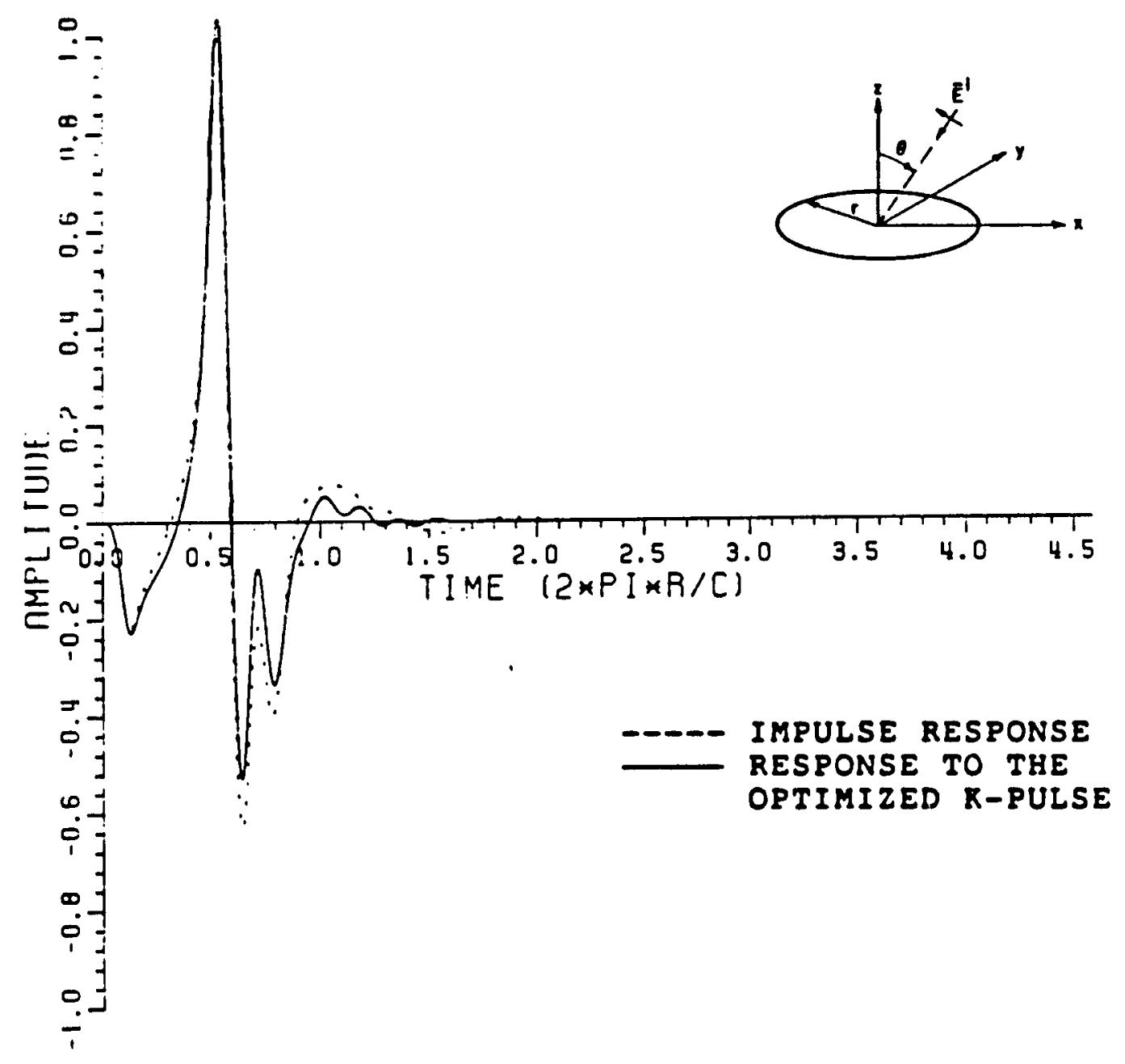

(a) No expansion of the amplitude scale.

Figure 3.29. Impulse response and response to the $K$-pulse shown in Figure 3.28 for the conducting circular disk at $\theta=45^{\circ}$, $\theta$-polarization with $\mathrm{kr}=0.16(0.16) 16$. 


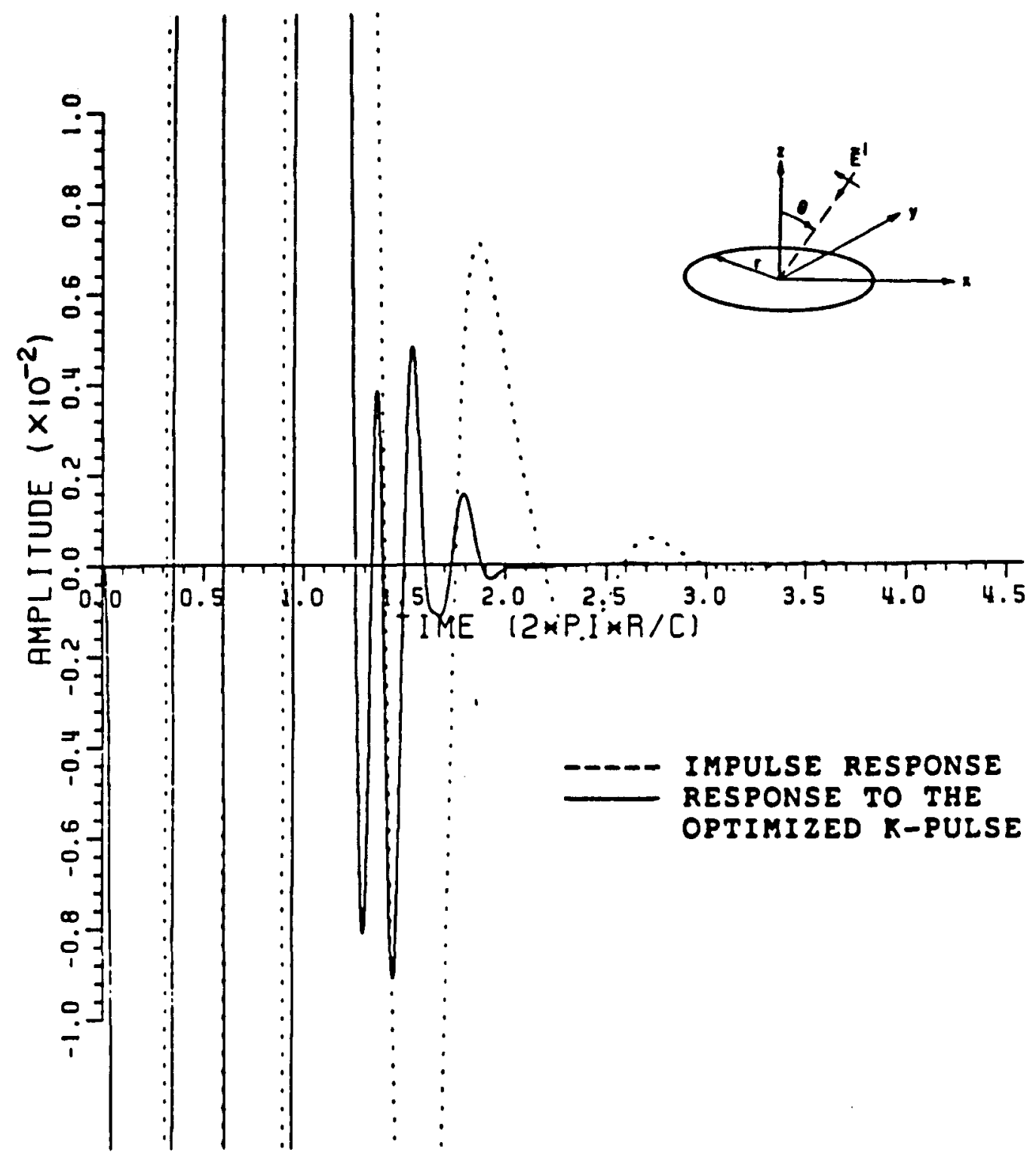

(b) The amplitude scale is expanded by 100 .

Figure 3.29. Continued. 


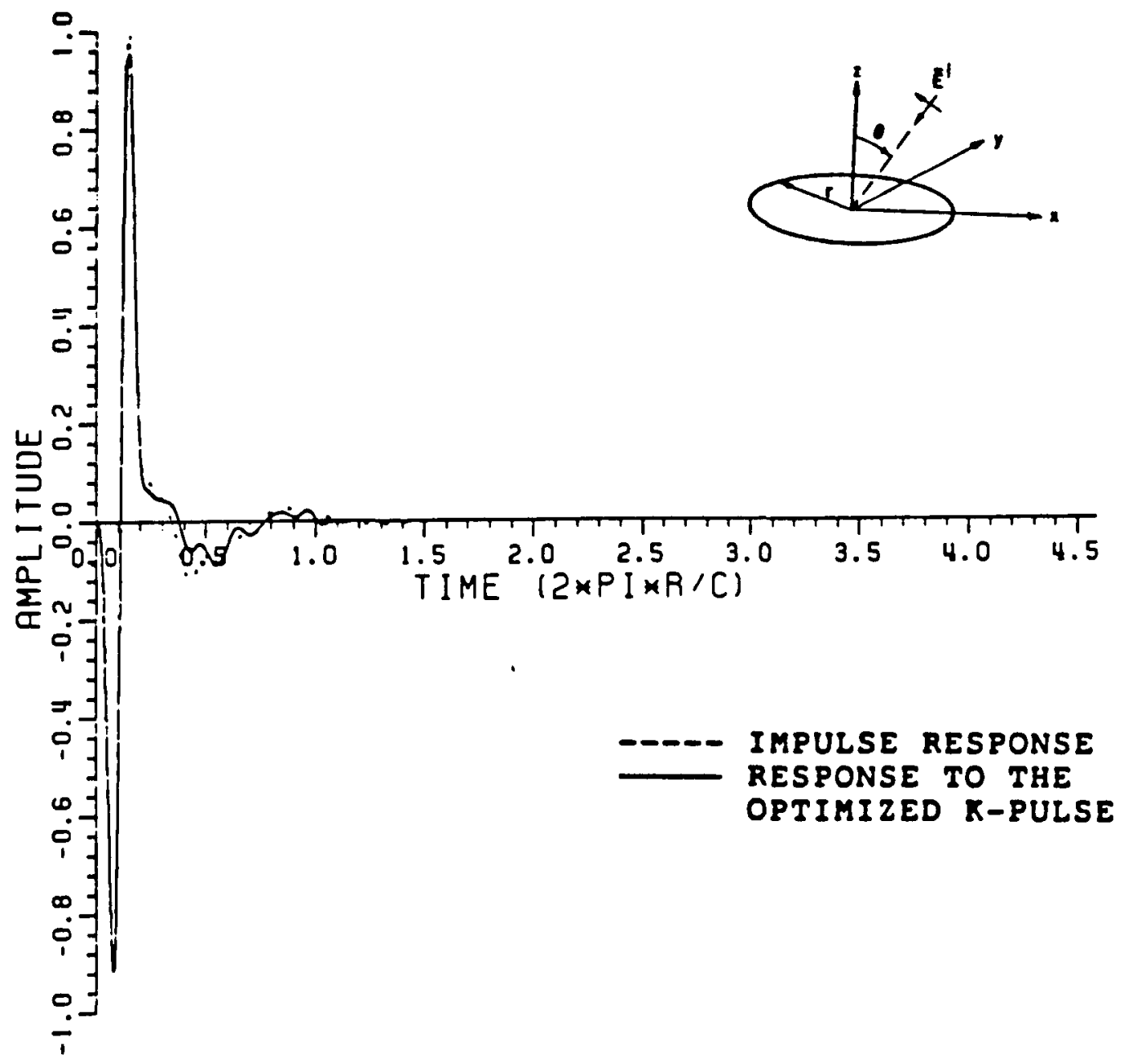

(a) No expansion of the amplitude scale.

Figure 3.30. Impulse response and response to the $K$-pulse shown in Figure 3.28 for the conducting circular disk at $\theta=0^{\circ}$, $\theta$-polarization with $\mathrm{kr}=0.16(0.16) 16$. 


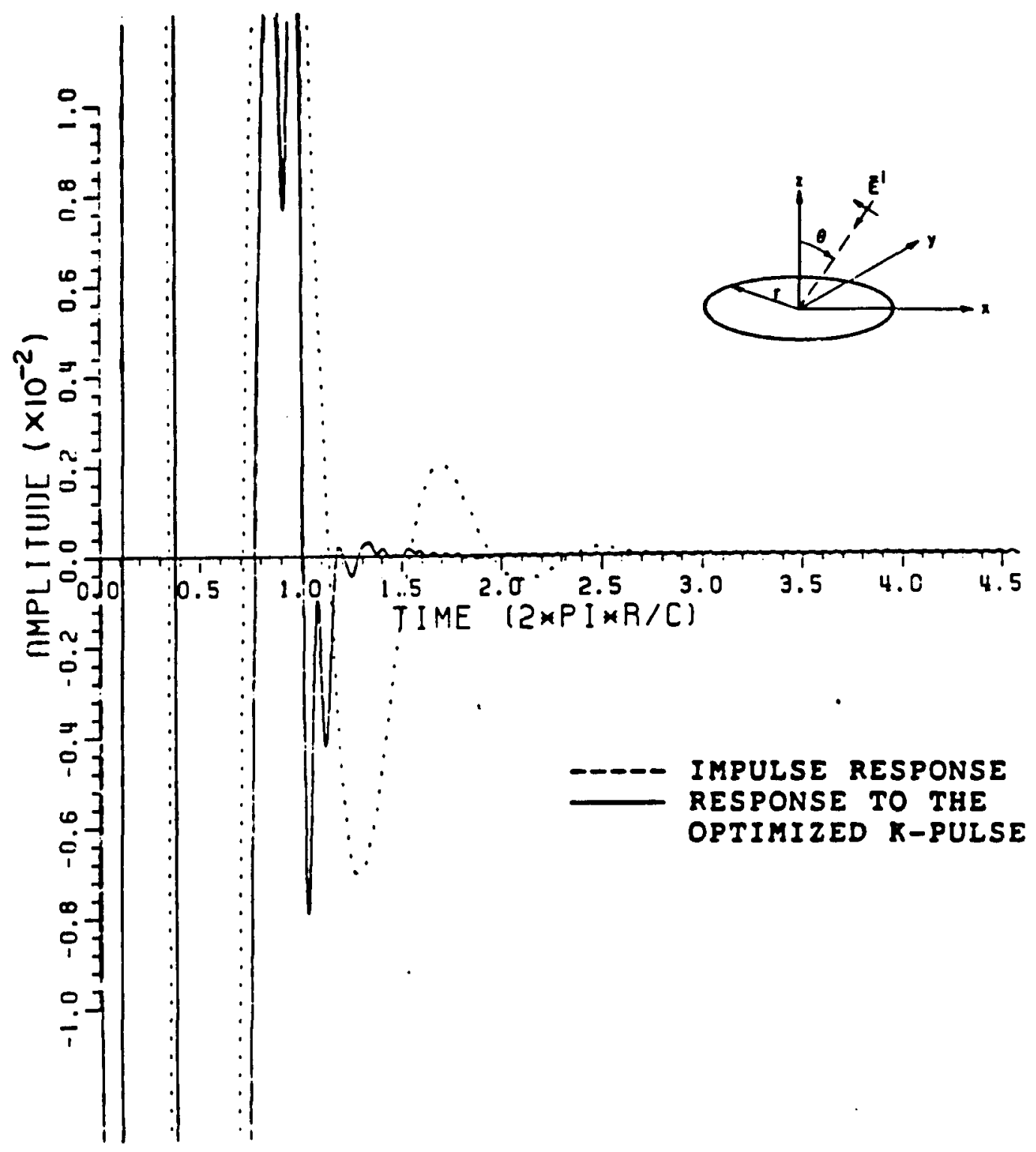

(b) The amplitude scale is expanded by 100 .

Figure 3.30. Continued. 


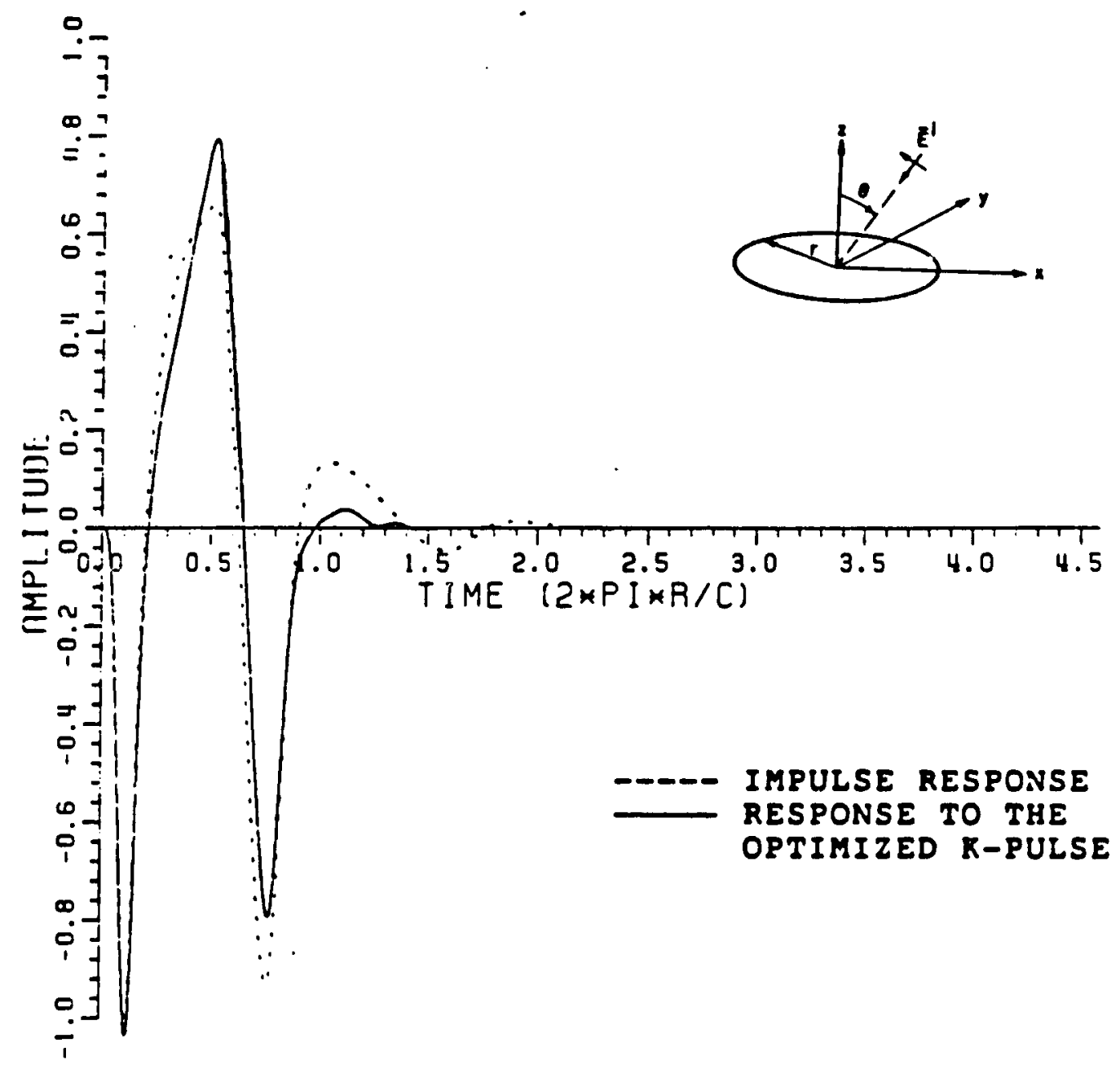

(a) No expansion of the amplitude scale.

Figure 3.31. Impulse response and response to the K-pulse shown in Figure 3.28 for the conducting circular disk at $\theta=45^{\circ}$, $\phi$-polarization with $\mathrm{kr}=0.16(0.16) 16$. 


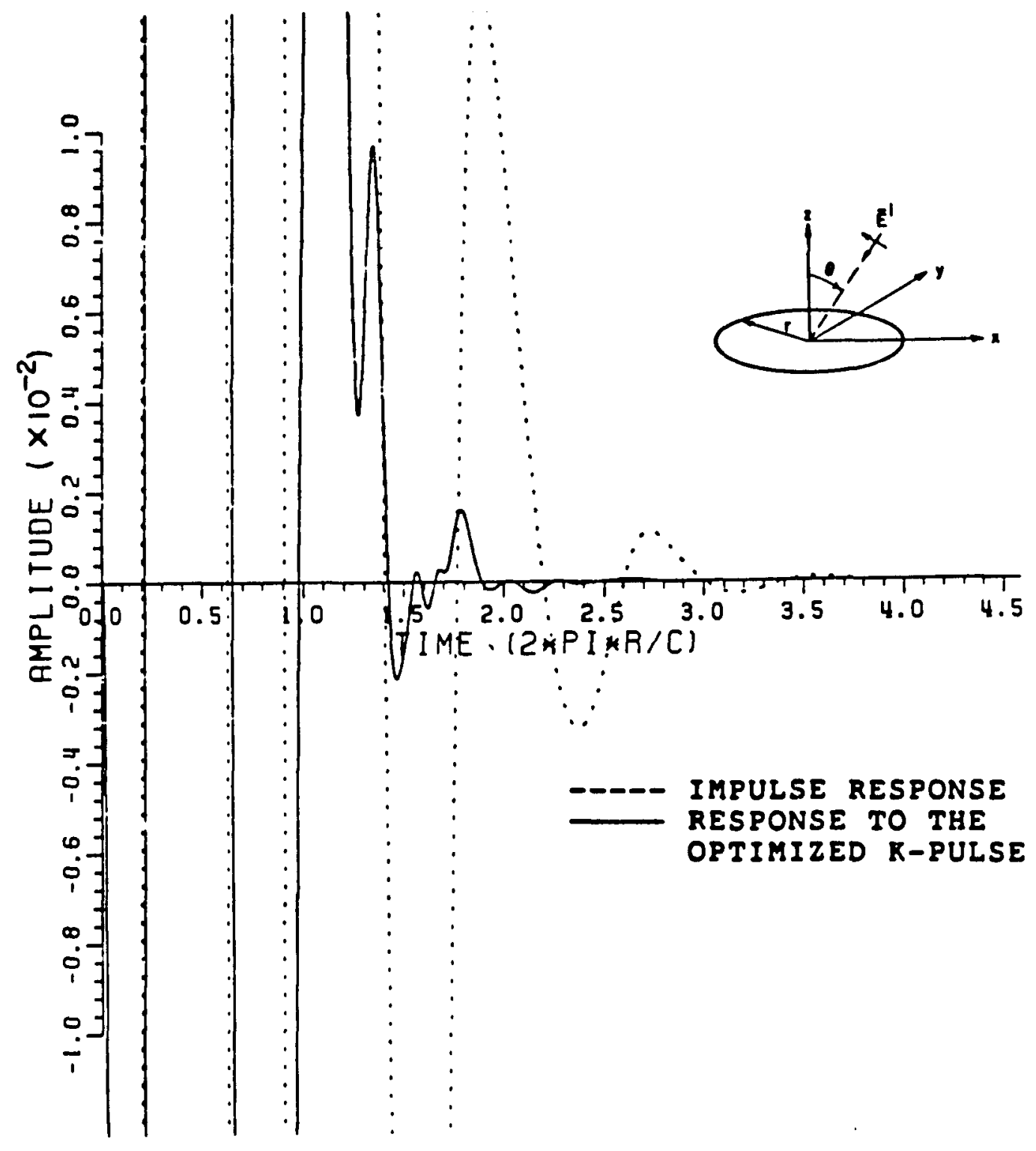

(b) The amplitude scale is expanded by 100 .

Figure 3.31. Continued. 


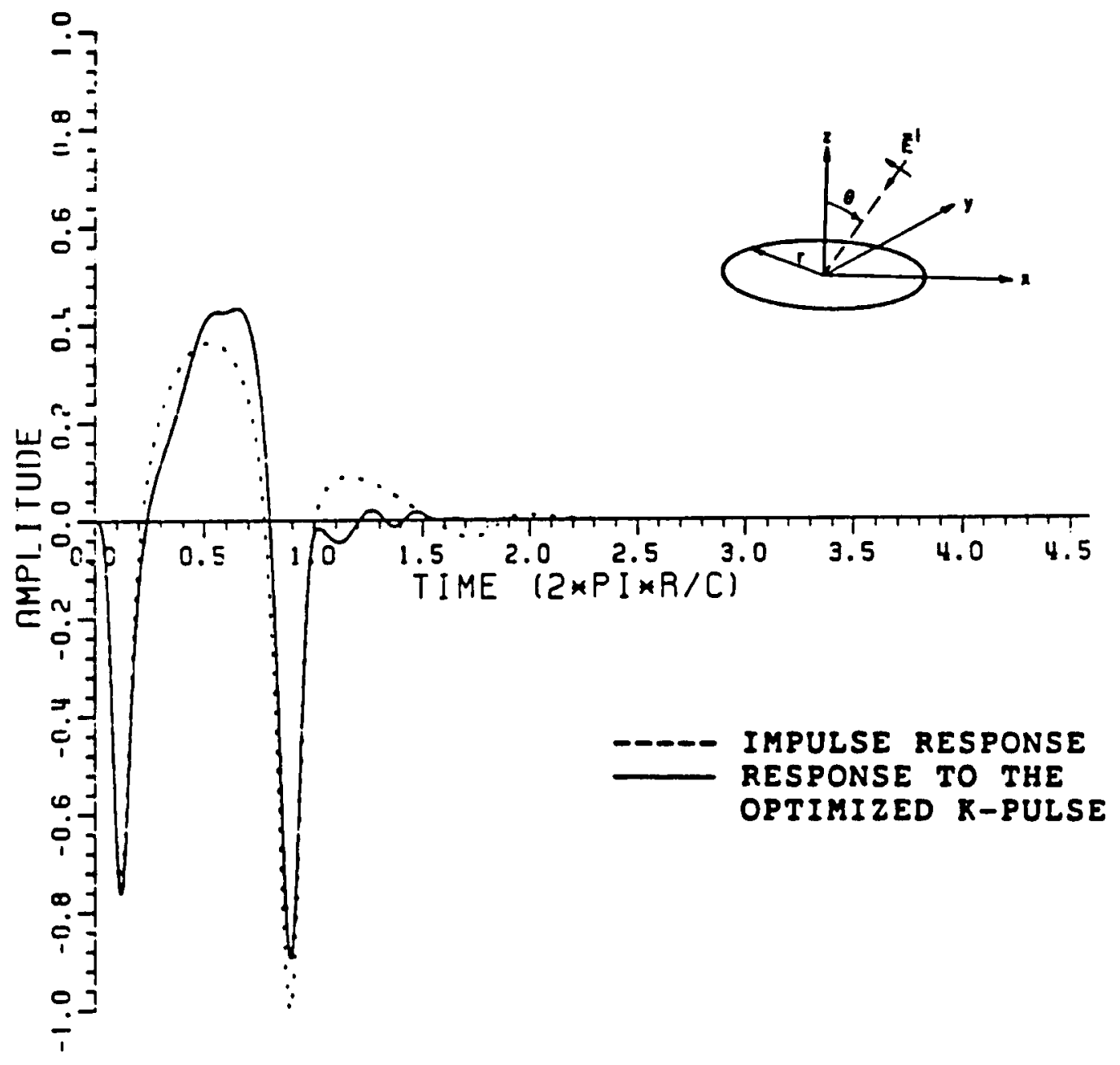

(a) No expansion of the amplitude scale.

Figure 3.32. Impulse response and response to the $K$-pulse shown in Figure 3.28 for the conducting circular disk at $\theta=90^{\circ}$, \$-polarization with $\mathrm{kr}=0.16(0.16) 16$. 


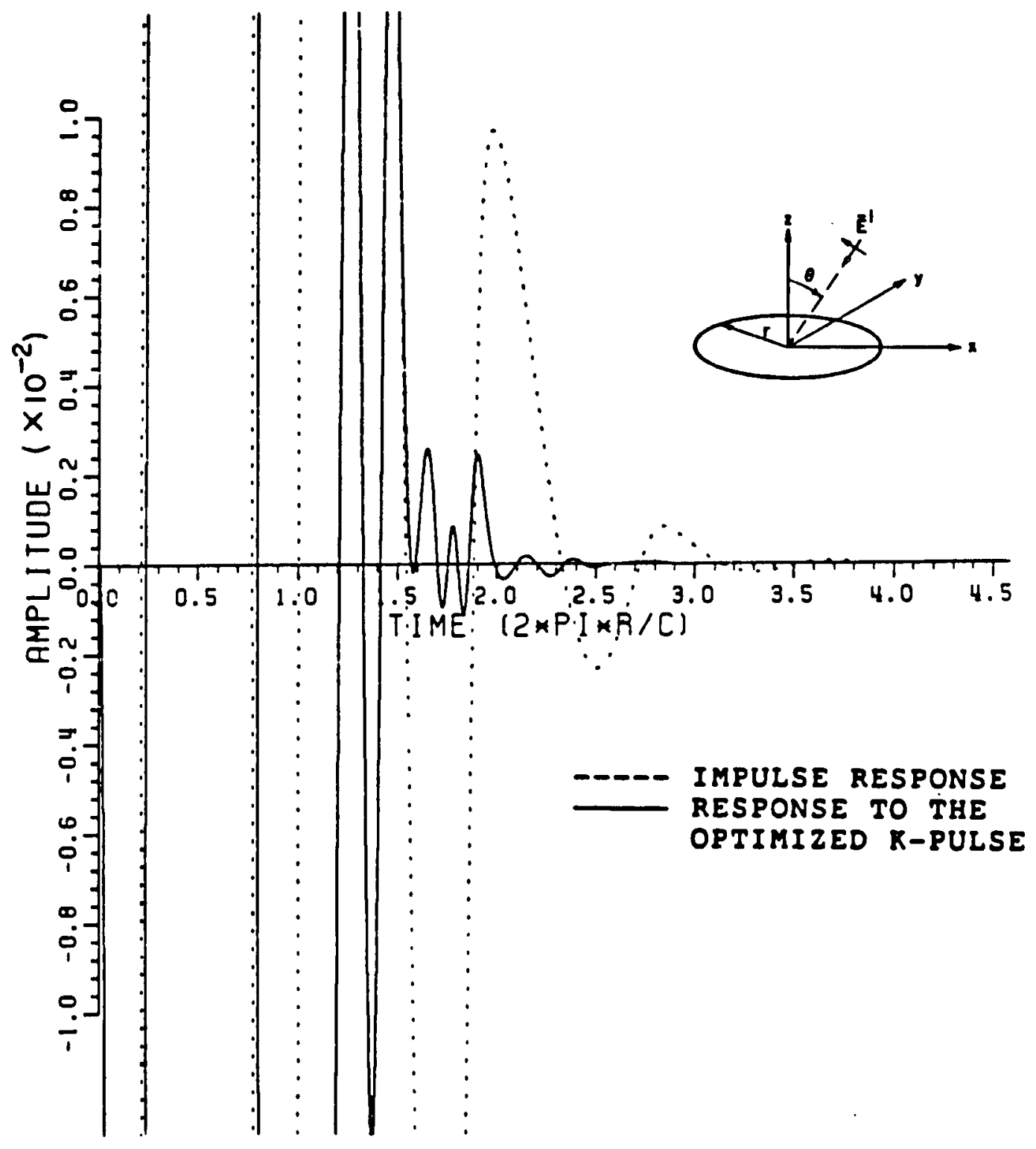

(b) The amplitude scale is expanded by 100 .

Figure 3.32. Continued. 


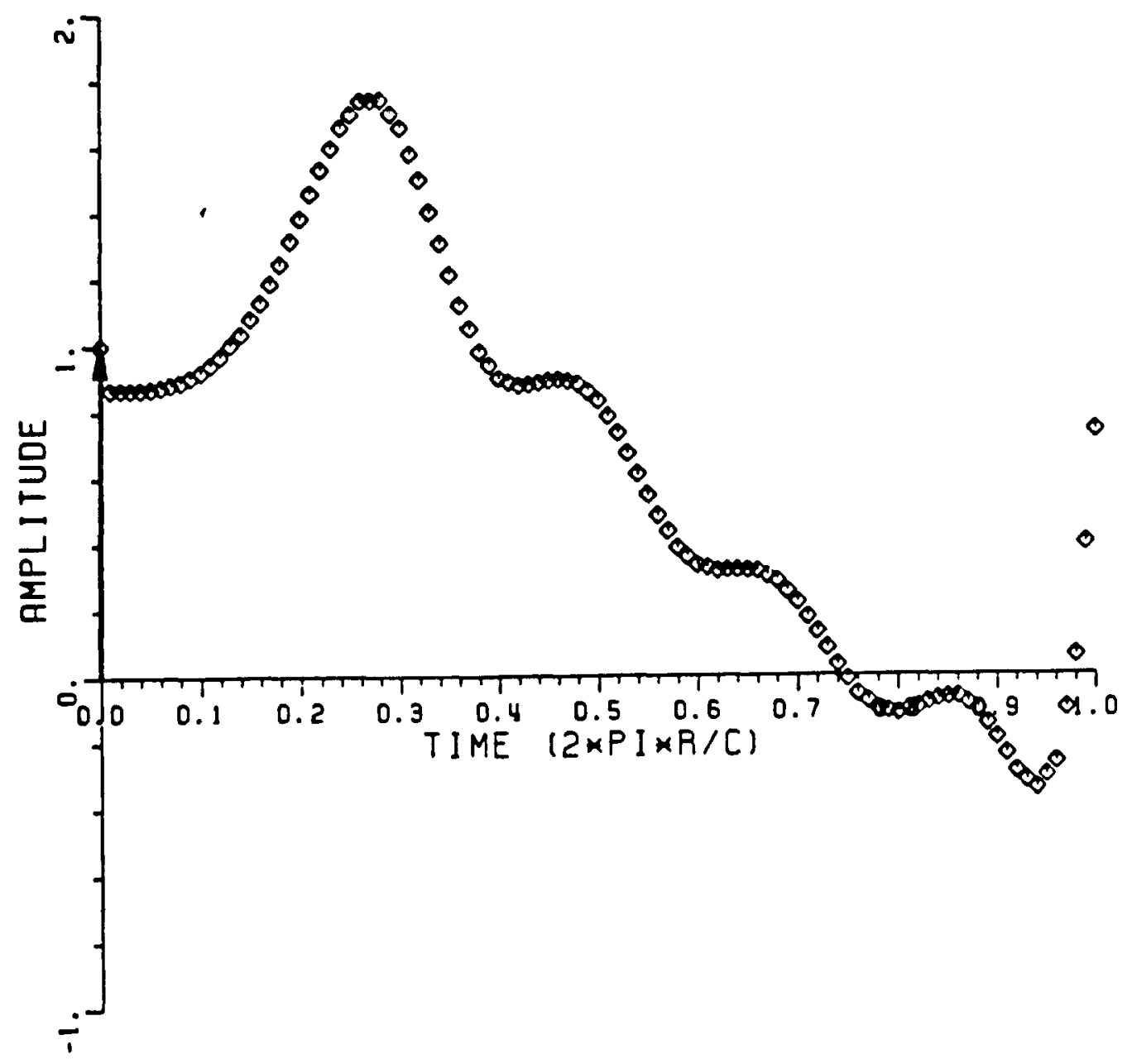

Figure 3.33. K-pulse obtained using the Fourier synthesized impulse response of the conducting circular disk at $\theta=45^{\circ}$, ф-polarization with $\mathrm{kr}=0.16(0.16) 16$. 


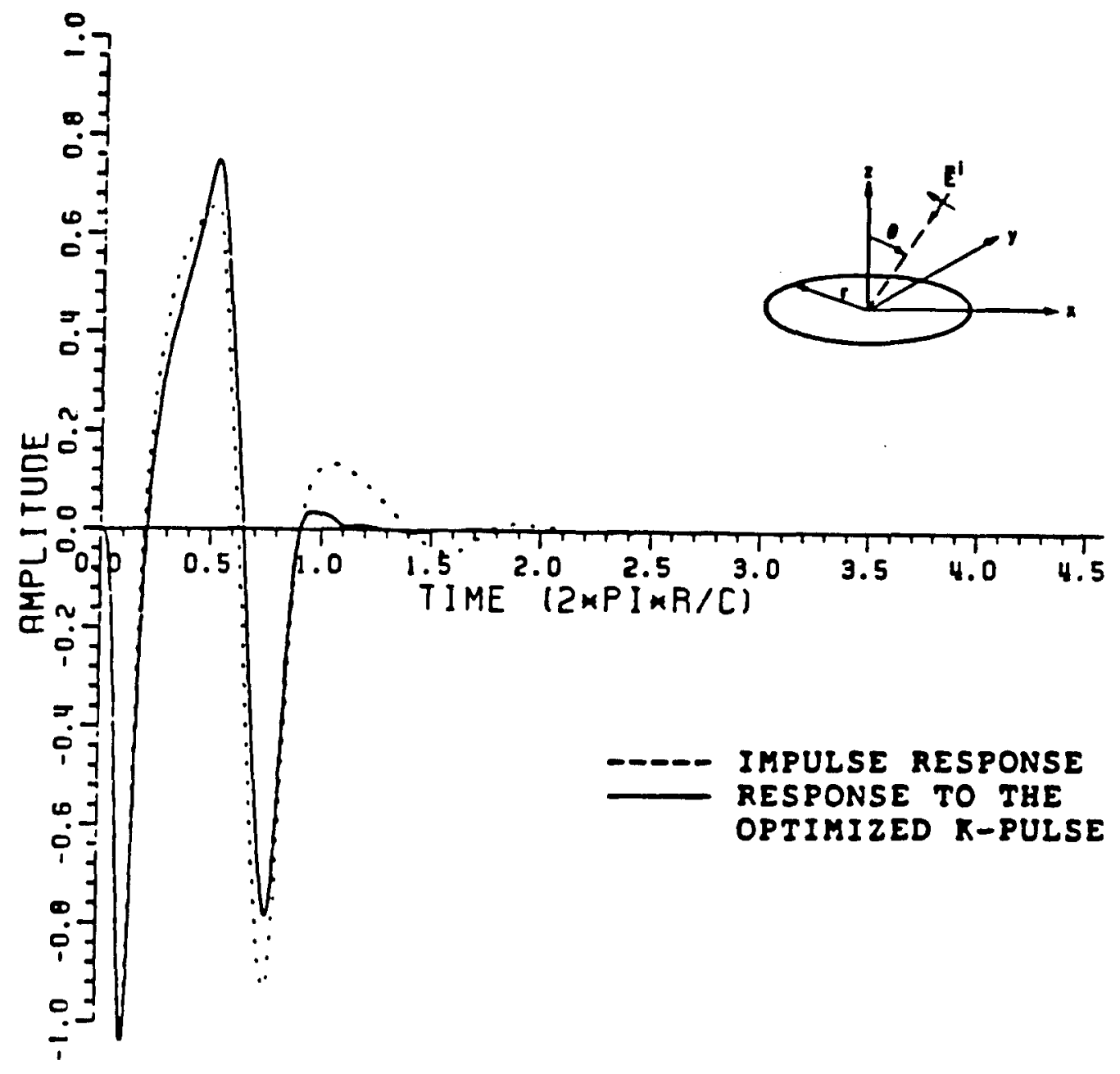

(a) No expansion of the amplitude scale.

Figure 3.34. Impulse response and response to the $K$-pulse shown in Figure 3.33 for the conducting circular disk at $\theta=45^{\circ}$, \$-polarization with $\mathrm{kr}=0.16(0.16) 16$. 


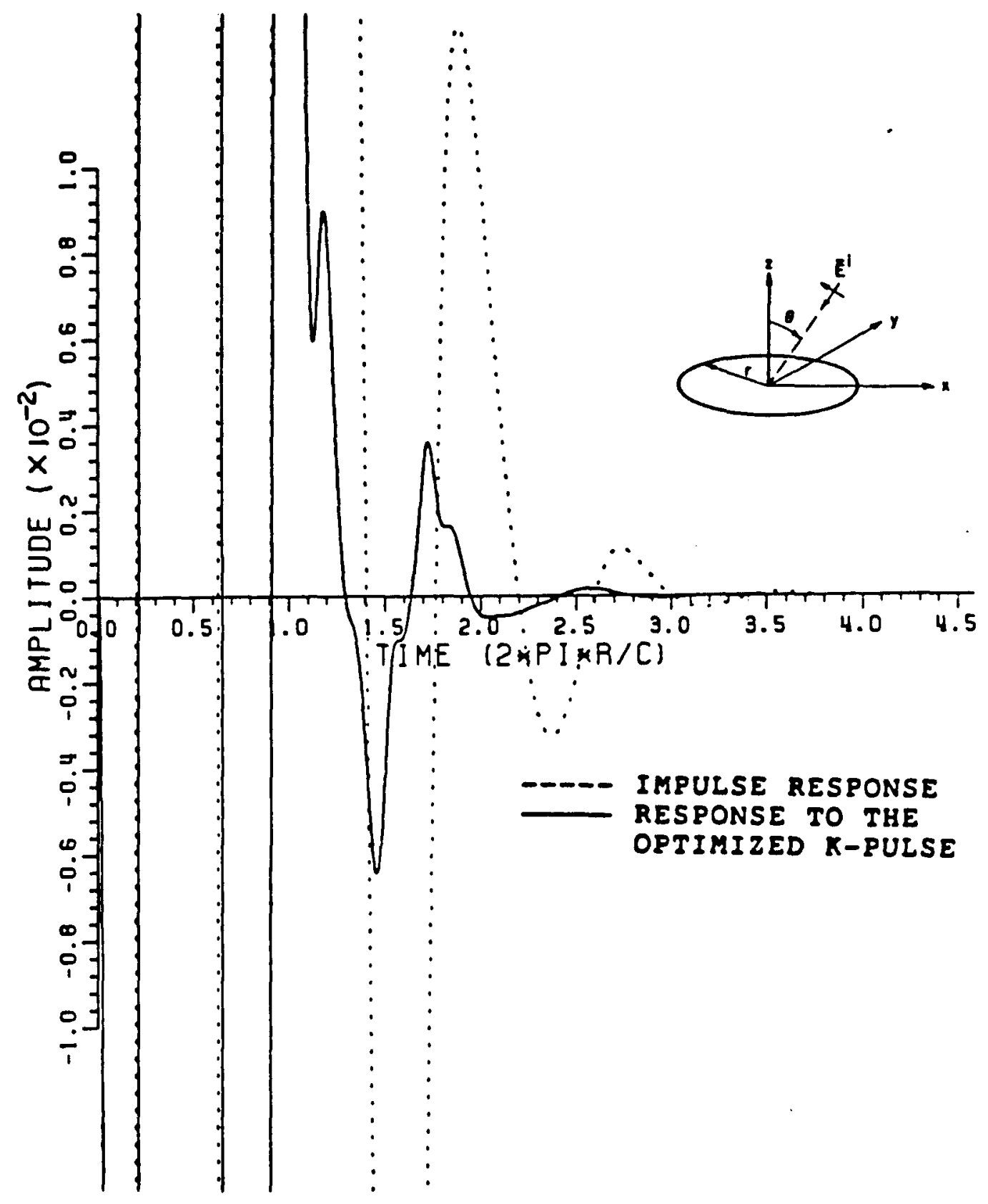

(b) The amplitude scale is expanded by 100 .

Figure 3.34. Continued. 


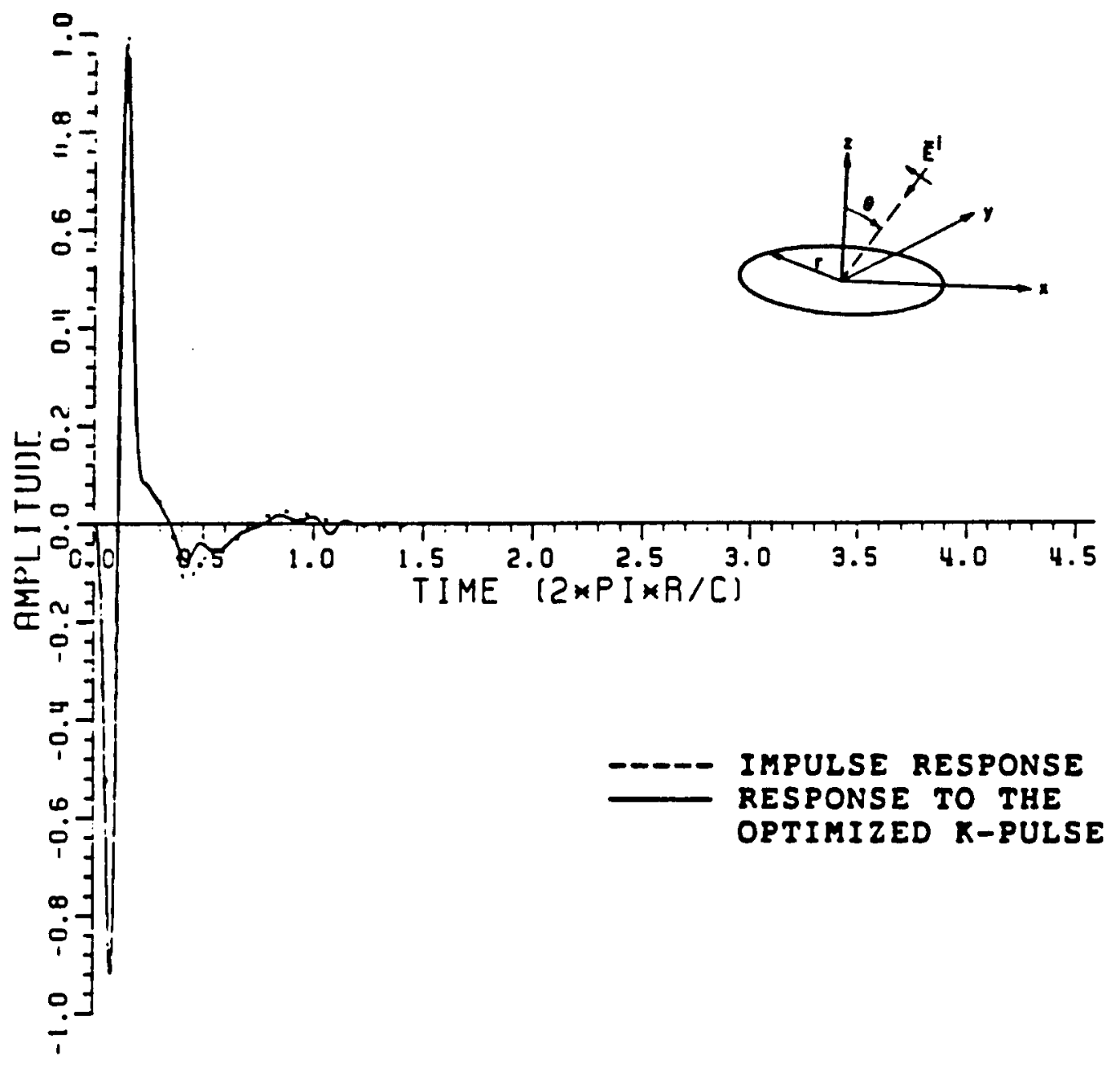

(a) No expansion of the amplitude scale.

Figure 3.35. Impulse response and response to the $K$-pulse shown in Figure 3.33 for the conducting circular disk at $\theta=0^{\circ}$, $\hat{\theta}$-polarization with $\mathrm{kr}=0.16(0.16) 16$. 


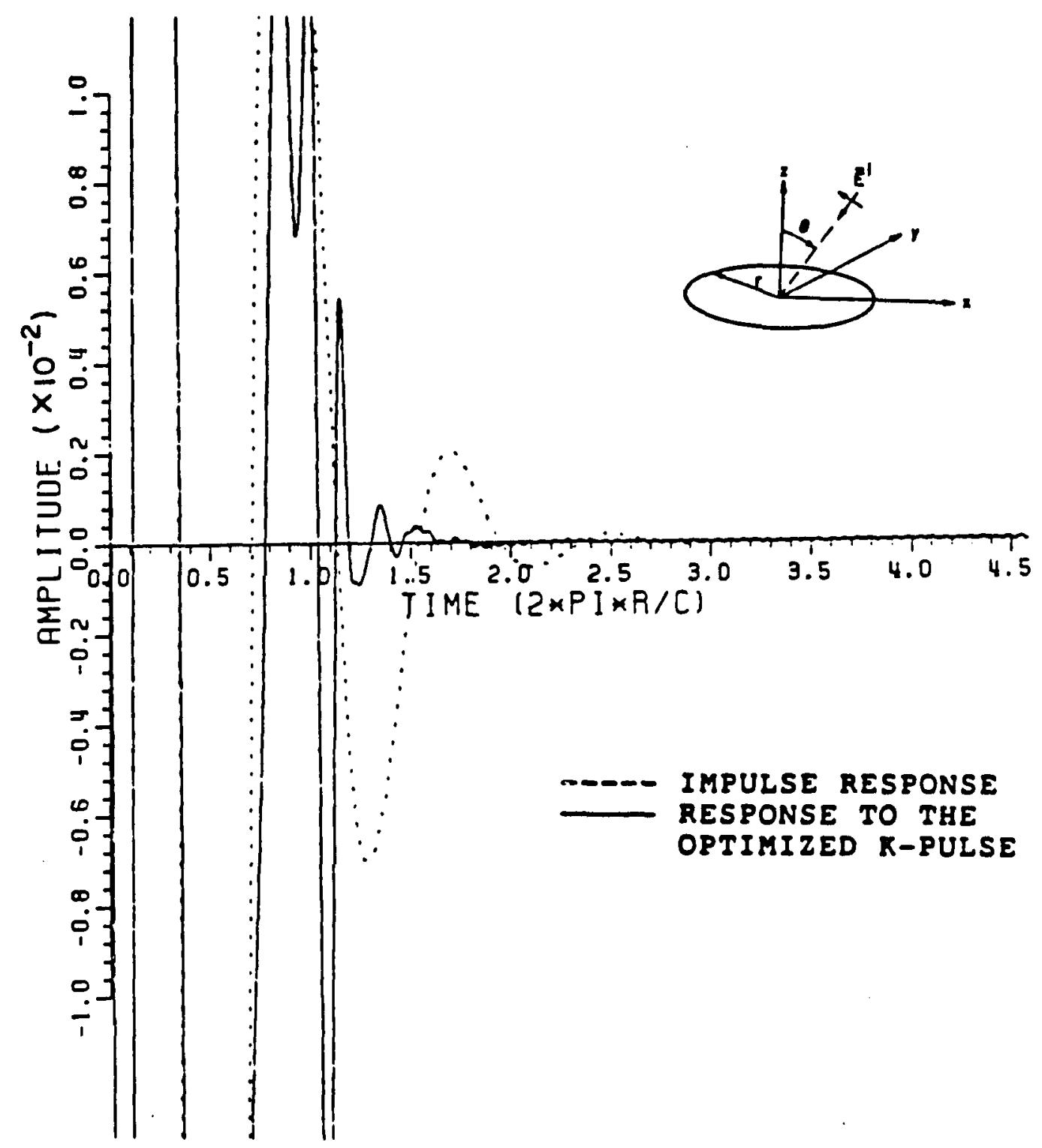

(b) The amplitude scale is expanded by 100 .

Figure 3.35. Continued. 


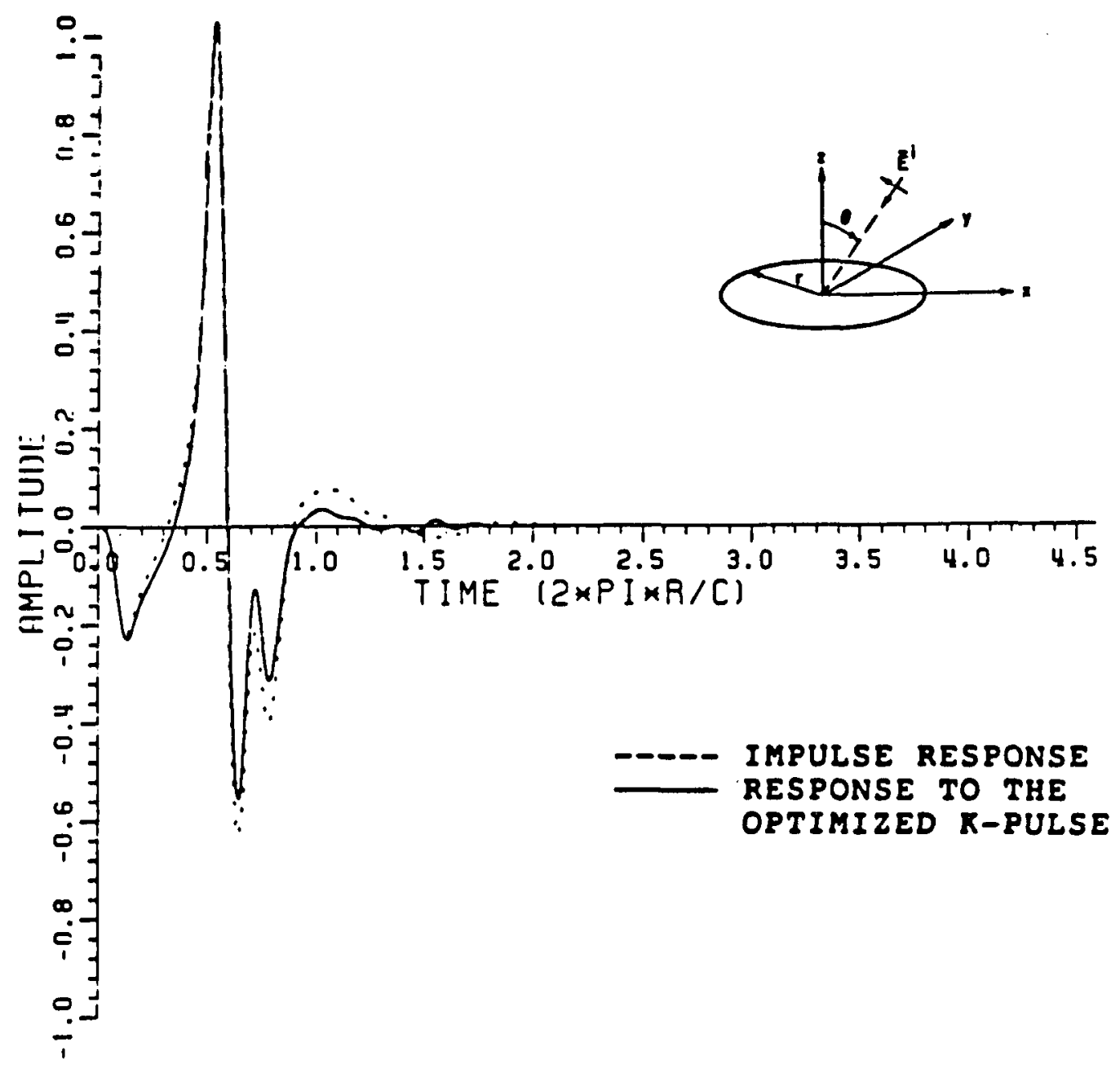

(a) No expansion of the amplitude scale.

Figure 3.36. Impulse response and response to the $K$-pulse shown in Figure 3.33 for the conducting circular disk at $\theta=45^{\circ}$, $\theta$-polarization with $\mathrm{kr}=0.16(0.16) 16$. 


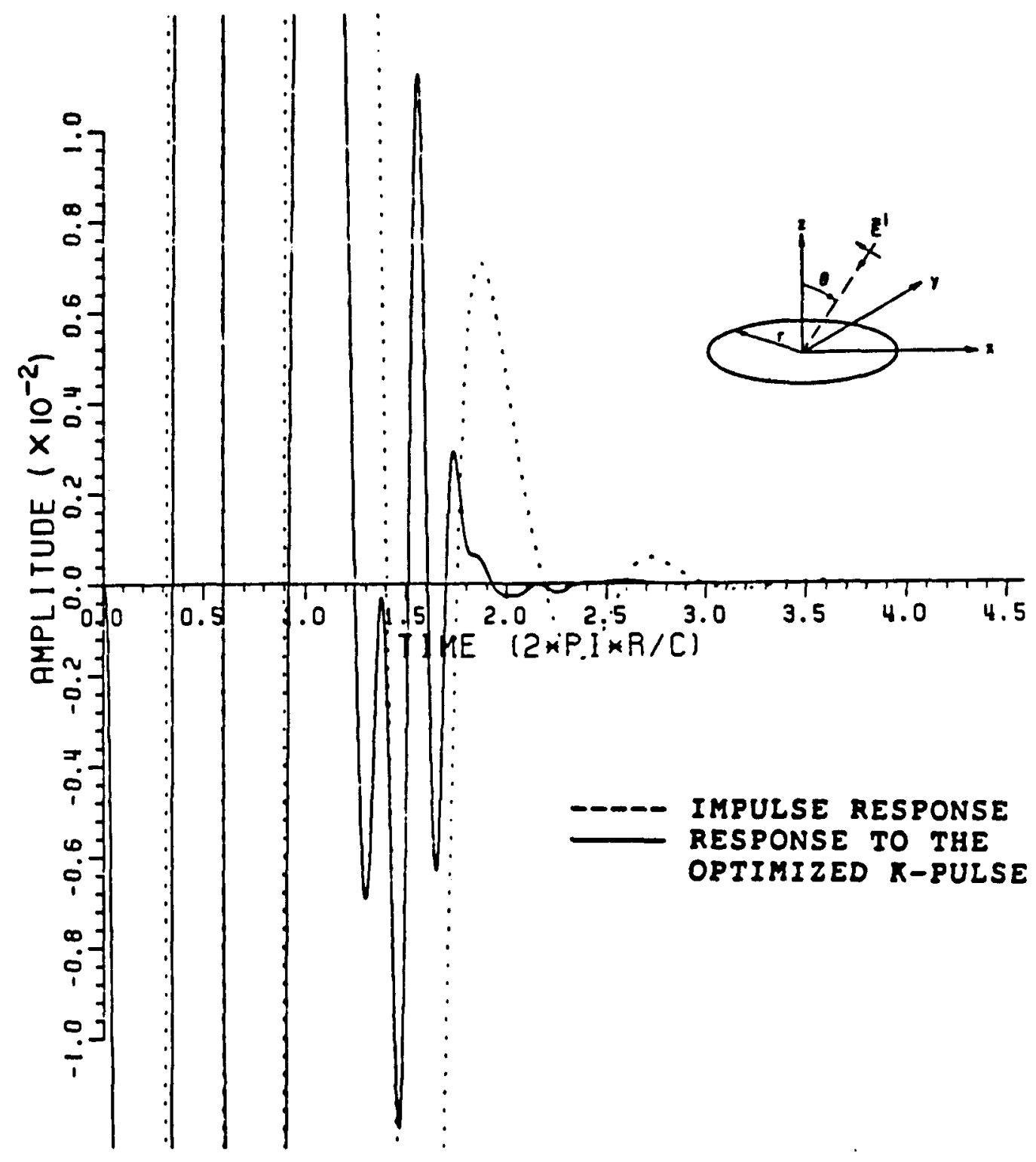

(b) The amplitude scale is expanded by 100 .

Figure 3.36. Continued. 


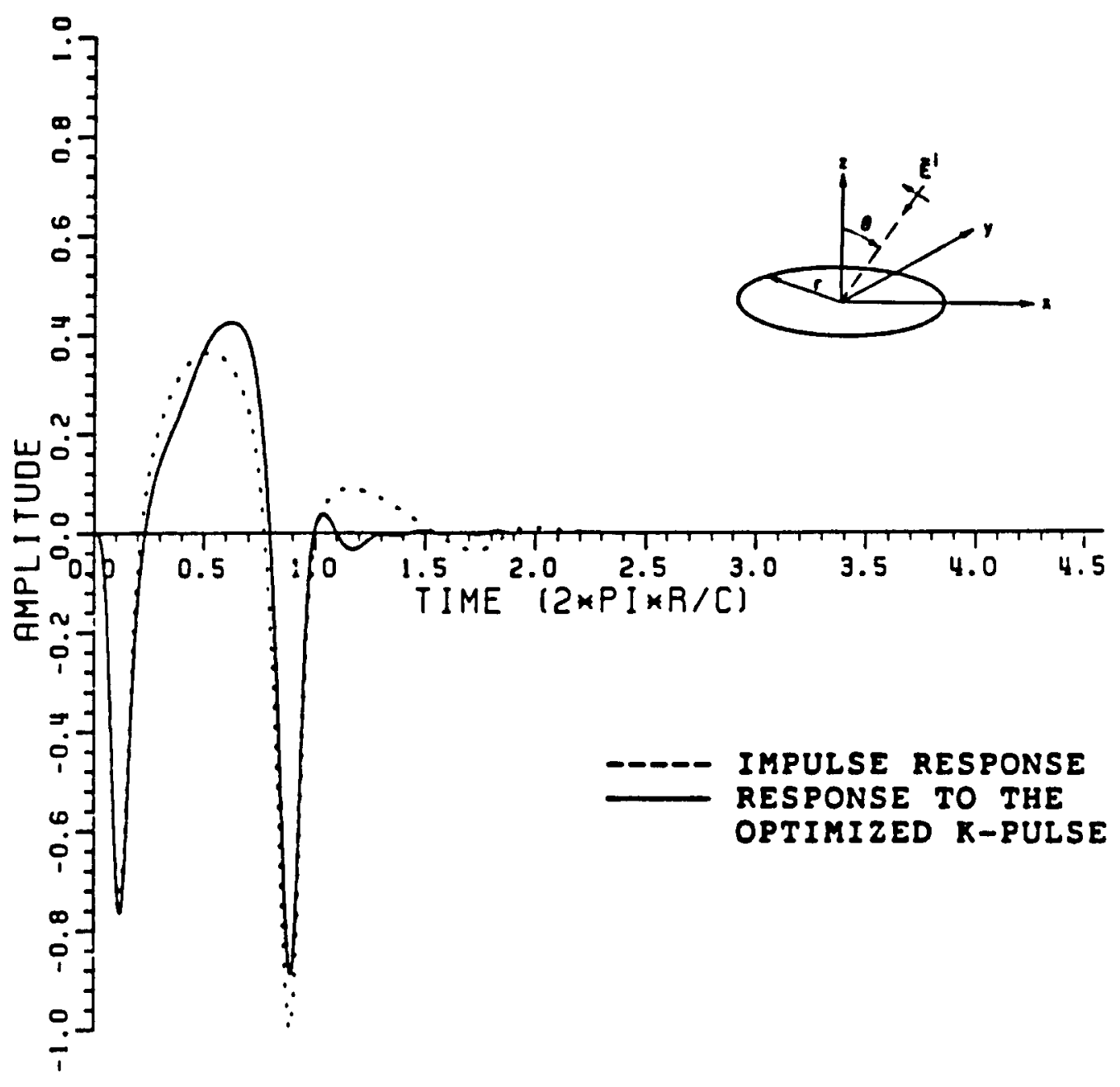

(a) No expansion of the amplitude scale.

Figure 3.37. Impulse response and response to the $K$-pulse shown in Figure 3.33 for the conducting circular disk at $\theta=90^{\circ}$, $\hat{\phi}$-polarization with $\mathrm{kr}=0.16(0.16) 16$. 


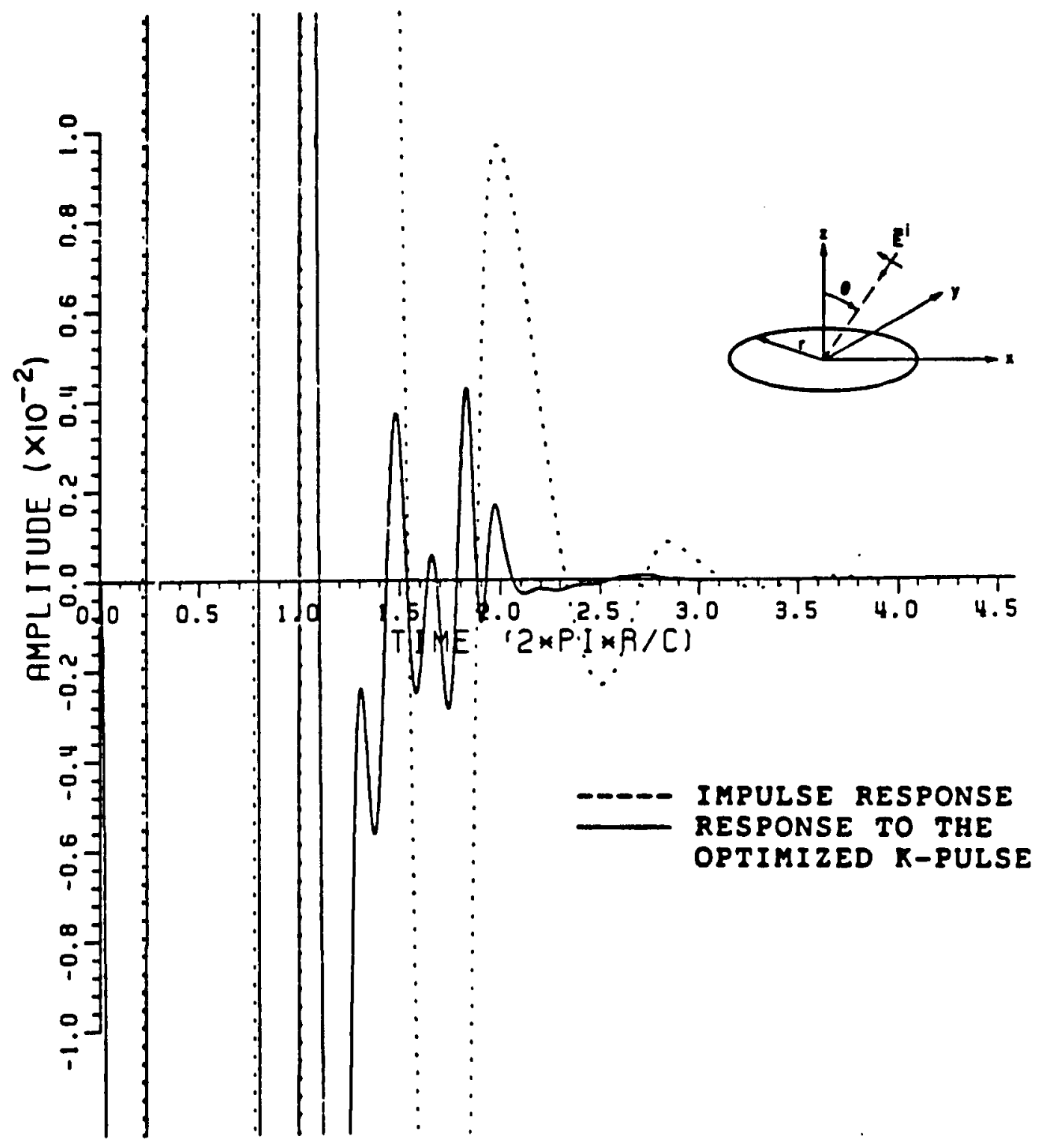

(b) The amplitude scale is expanded by 100 .

Figure 3.37. Continued. 


\section{CHAPTER IV}

\section{ESTIMATION OF THE DURATION OF THE K-PULSE}

- One of the necessary conditions for a unique $K$-pulse is that fts duration must be minimal [2]. Consider the following example. Let $k(t)$ be the K-pulse of a target with duration T. Now let

$$
k_{1}(t)=k(t)+\alpha k\left(t-T_{1}\right)
$$

which has a duration of $T+T_{1} ; a$ and $T_{1}$ are arbitrary constants. This K-pulse will also cancel the poles of the target, because the Laplace transform of $k_{1}(t)$ gives

$$
K_{1}(s)=K(s)\left[1+\alpha e^{-s T_{1}}\right]
$$

and $K_{1}(s)$ has not only the zeros of $K(s)$ but also,

$$
s_{n}=\left(1 / T_{1}\right)[\ln (\alpha)+j(2 n+1) \pi]
$$

where $n$ is an integer. If $T_{1}$ is not zero, then any value of a will still cancel the poles of the target. As there are an infinite number of choices for $\alpha$, there are also an infinite set of functions that will cancel the poles. Consequently, to have a unique K-pulse, its duration is of minimal is a necessary condition. For simple geometries, the duration of the $K$-pulse $(T)$ is chosen to be the circumnavigational path length over the velocity. For example, for the circular loop, sphere and circular disk the $K$-pulse duration is $2 \pi r / c$, where $r$ is the radius. 
In other words, $T$ corresponds to the circumference over the velocity of the wave. For the straight wire and the grounded dielectric slab $T=2 L / v$, where $L$ is the length of the wire or the thickness of the slab. For more complicated geometries, this type of interpretation is not easy. Another approach is needed, and one method is described in this chapter.

The difference between the imaginary parts of neighboring poles for a plane wave normally incident on the grounded dielectric slab is $j v \pi / L$. The time corresponding to this

$$
\Delta \omega=v \pi / L
$$

difference frequency is $T=2 L / v$. Table 4.1 lists the difference between the imaginary parts of consecutive poles for the five targets discussed in the previous chapter. (For the convenience of the reader, 211 tables and figures of Chapter IV are grouped together at the end of the chapter.) The difference in radian frequency for the wire

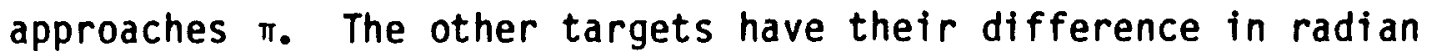
frequency approaching $2 \pi$. The time corresponding to this difference of frequency is $L / C$ for Equation $(3.2) ; 2 L / C$ for the wire; $2 \pi r / C$ for the loop, sphere and disk. These are all the proper values for the duration of the K-pulse. Calculated from Table 4.1, the times corresponding to the first differences are $0.952 \mathrm{~L} / \mathrm{C}, 2.014 \mathrm{~L} / \mathrm{C}, 1.975 \pi \mathrm{r} / \mathrm{C}, 2.013 \pi \mathrm{r} / \mathrm{C}$, $1.863 \pi r / C$, for the low 0 model [Equation (3.2)], wire, loop, sphere and disk, respectively. They are very close to the true K-pulse duration. These are all summarized in Table 4.2. It appears the K-pulse duration is related to the density of the poles. 
Since the main concept in this dissertation is based on minimization of the energy due to the natural resonances, the $K$-pulse obtained with any duration longer than the proper duration will contain the dominant pole information. More generally, the K-pulse obtained with non-minimal duration will have the following Laplace transform:

$$
K_{m n}(s)=K(s) G_{m}\left(s, T_{n}\right)
$$

where $G_{m}(s)$ is any entire function of exponential type for variable $s$, and $T_{n}$ is the duration of $g_{m}(t)$, which is the inverse Laplace transform of $G_{m}\left(s, T_{n}\right)$. Note that " $m$ " and " $n$ " are used to represent the infinite number of entire functions and durations. In other words, the poles associated with $K(s)$ will always remain in $K_{m n}(s)$ as the guess of the K-pulse duration is changed. They would remain unchanged until the guess of the K-pulse duration is less than the proper K-pulse duration. When the guess of the $K$-pulse duration is less than the proper K-pulse duration, either the minimization process will not converge or the pole strings will be totally different from those of the true K-pulse. Using this information and the density of the poles, the duration of K-pulse can be estimated. Also if $T_{n}=0$, then $G_{m}(s)$ is a real constant, an exponential function of $s$, or a polynomial of $s$. All of these will not affect the result of estimating the duration of the $K$-pulse because of the $K$-pulse model employed in this dissertation. The constant, of course, will not affect the pole string obtained. The exponential function of $s$ will give a time shift for $k(t)$, but the $k$-pulse model is forced to start at time zero. Besides, this pole string will always 
have the real parts being zero and can be ignored. Lastly, a polynomial of $s$ for $G_{m}(s)$ cannot occur because only impulsive singularities are allowed in the model.

The optimization process is started with a reasonable guess of the duration of $\mathrm{K}$-pulse $\left(\mathrm{T}_{\mathrm{gl}}\right)$. It is better for this guess to be longer than the true duration as opposed to a shorter duration. A reasonable estimate seems to be the period of the damped sinusold exhibited by the dominant natural resonance of the target at late time. After the first guess $K$-pulse $\left[k_{g 1}(t)\right]$ is obtained, the value of the $k$-pulse duration guess $\left(T_{g 1}\right)$ is increased to some other guess $\left(T_{g_{2}}\right)$ and its corresponding $k$-pulse $\left[k_{g 2}(t)\right]$ is obtained. The dominant pole strings are compared. If the two strings are totally different, then the second guess $\left(T_{g 2}\right)$ needs to be increased to a third guess $\left(T_{g_{3}}\right)$ to obtain a corresponding $k$-pulse $\left[k_{g_{3}}(t)\right]$. This process is repeated unt 11 the two consecutive pole strings have some similar poles. Then the difference of the radian frequency $(\Delta \omega)$ between consecutive and more dominant poles but common in both strings are calculated. The value of a better guess for the duration of the K-pulse (T) is then $2 \pi / \Delta \omega_{0}$

The above procedure is checked with the five targets discussed in the previous chapter. The high $Q$ structures are discussed before the low $Q$ structures. The density of samples for the continuous portion of the K-pulse is taken to be 50 samples/T. From experience, the choice of 50 samples/T seems to be a good starting point for the optimization process. The first and second guesses are assumed to be 1.5T and 2T, respectively. The third guess for the K-pulse duration is $0.5 T$. This 
third guess is used to confirm that $T$ is the proper K-pulse duration. To start the optimization process all the inftial guesses of the unknowns in the K-pulse model are specified as unity.

The thin straight wire is the first example to be discussed. The duration of the first guess $K$-pulse is $3 L / C\left(T_{w 1}\right)$. The duration of the $K$-pulse response is $5 \mathrm{~L} / \mathrm{C}\left(T_{R}\right)$ and the cutoff time for the integration is $9 L / C\left(T_{C}\right)$. The bandwidth of the Fourier synthesized impulse responses used is $K L=0.08 \pi(0.08 \pi) 8 \pi$. After twenty-five steps of optimization using the impulse response of the straight wire at $\phi=30^{\circ}$, $\hat{\phi}$-polarization, the $K$-pulse $\left[k_{w 1}(t)\right]$ for the first K-pulse duration guess is shown in Figure 4.1. The energy in the $K$-pulse response outside 4L/C is very small as shown in Figure 4.2. This K-pulse response is different from what is shown in Figure 3.14 , because the durations of the $k$-pulses are different. $k_{w 2}(t)$ in Figure 4.3 is derived similarly except its duration $T_{W 2}$ is chosen to be $4 L / C$ and only ten steps of optimization are specified. The $K$-pulse response of the wire at $\phi=30^{\circ}$ is shown in Figure 4.4. The energy content after $5 \mathrm{~L} / \mathrm{C}$ is again very small. Comparing the zero strings of $k_{w 1}(t)$ and $k_{w 2}(t)$ (Table 4.3), the first approximation of the duration of the K-pulse (T) is calculated to be $2.009 \mathrm{~L} / \mathrm{C}$, which is very close to the true $T(2 \mathrm{~L} / \mathrm{C})$. If the duration of the $K$-pulse is chosen to be $2 \mathrm{~L} / \mathrm{C}$, the $\mathrm{K}$-pulse as in Figure 3.13 will result. The responses for the straight wire at the different aspect angles will be as shown in Figures 3.14, 3.15, 3.16. The energy of the $K$-pulse responses in late time at the various angles will be very small. For a final check, the guess duration of the 
K-pulse $\left(T_{w 3}\right)$ is chosen to be $L / C$. After twenty-five steps of optimization, no reduction in energy is shown outside the K-pulse response as shown in Figure 4.5. Not surprisingly, the "K-pulse" obtained (Figure 4.6) does not have any zero similar to the K-pulses obtained previously. The reason why K-pulse is in quotations for Figure 4.6 is the waveform obtained no longer contains the natural resonance information of the target.

The loop exhibits results similar to those obtained for the wire. The first guess of the K-pulse duration $\left(T_{\ell 1}\right)$ is $3 \pi r / c$. The duration of the $K$-pulse response $\left(T_{R}\right)$ is $8 \pi r / C$ and the cutoff time $\left(T_{C}\right)$ for integration is $12 \pi r / c$. The bandwidth of the Fourier synthesized impulse response used is $k r=0.02 \pi(0.02 \pi) 4 \pi$. After twenty-five steps of optimization, the first guess $K$-pulse $\left[k_{\ell 1}(t)\right]$ is obtained as shown in Figure 4.7. The impulse response used is at $\theta=45^{\circ}, \hat{\theta}$-polarization. The corresponding K-pulse response is shown in Figure 4.8. The energy content outside $4 \pi r / c$ is minimized. After ten steps of optimization, the second guess $k$-pulse $k_{\ell 2}(t)$ as shown in Flgure 4.9 is obtained with the duration of the K-pulse $\left(T_{\ell 1}\right)$ increased to $4 \pi r / C\left(T_{\ell 2}\right)$. The response due to $k_{\ell 2}(t)$ is contrasted with the impulse response in Figure 4.10. The signal amplitude is small at the late time. The zeros derived from both K-pulses are compared in Table 4.4. The first approximation to the K-pulse duration $(T)$ is calculated to be $1.983 \pi r / c$, which is very close to the true duration of $2 \pi r / c$. Using a $K$-pulse duration of $2 \pi r / c$, Figure 3.17 shows the $K$-pulse with the proper duration. Then the responses of the loop at different angles to this 
K-pulse are shown in Figures 3.18 to 3.21. All the K-pulse responses have very little energy remaining. If the duration of the K-pulse is chosen to be $\pi r / c$, after twenty-five steps of optimization, no minimization of energy is achieved as shown in Figure 4.11, indicating the choice of $T$ is wrong. Again the "K-pulse" (Figure 4.12) obtained has no dominant zero similar to any of the dominant zeros obtained from the other three K-pulses.

Next the discussion turns to the low 0 structures - the low 0 model [Equation (3.2)], the sphere, and the disk. The method of minimization of the energy at late time is not expected to work well for the low 0 structures, as the energy contributed by their natural resonances is small even before the optimization begins. Nevertheless, it is interesting to see how the method to obtain the K-pulse duration behaves for the low Q structures. The impulse response used for Equation (3.2) has a bandwidth of $k L=0.05 \pi(0.05 \pi) 100 \pi$. The initial guesses for the $K$-pulse duration $\left(T_{e 1}\right)$, the $K$-pulse response duration $\left(T_{R}\right)$ and the cutoff time $\left(T_{C}\right)$ for integration are $1.5 \mathrm{~L} / \mathrm{C}, 0.5 \mathrm{~L} / \mathrm{C}, 2.92 \mathrm{~L} / \mathrm{C}$, respectively. After ten steps of optimization, the first $k$-pulse $k_{e l}(t)$ as shown in Figure 4.13 is obtained. The $K$-pulse response is shown in Figure 4.14. Increasing the K-pulse duration $\left(T_{e l}\right)$ to $2 L / C\left(T_{e 2}\right)$, the second $K$-pulse $k_{e 2}(t)$ as shown in Figure 4.15 is obtained after ten steps of optimization. The response to $k_{e 2}(t)$ is shown in Figure 4.16. Comparing the dominant zeros of $k_{e 1}(t)$ and $k_{e 2}(t)$ (Table 4.5), the first approximation to the $K$-pulse duration is calculated to be $0.948 \mathrm{~L} / \mathrm{C}$ which compares well with the true value of $L / C$. Using a value of $L / C$ for the 
$K$-pulse duration, the $K$-pulse as shown in Figure 3.11 will results, and response of the model to this $K$-pulse is shown in Figure 3.12. The zeros of this $\mathrm{K}$-pulse compare well with the poles of the model. Figure 4.17 shows the "K-pulse" with the guess of $K$-pulse duration ( $T_{e} 3$ ) being $0.5 \mathrm{~L} / \mathrm{c}$. The $\mathrm{K}$-pulse response as shown in Figure 4.18 shows the optimization process is walking in the proper direction. But a comparison of the zeros associated with Figure 4.17 and the other K-pulses for Equation (3.2) shows that these zeros obtained with K-pulse duration of $0.5 \mathrm{~L} / \mathrm{C}$ are not even close.

For the next two low $?$ structures, the duration of the K-pulse response must be known very accurately, as the energy contributed by the natural resonances in the late time is quite small. If the K-pulse response duration $\left(T_{R}\right)$ is chosen too large, the contribution can only come from the first complex pole-pair. The better is the estimate of the duration of the $\mathrm{K}$-pulse response; the better is the number of real poles avatlable. The amplitude of the K-pulse and impulse responses for these two targets are shown with a magnification of 100 . The number of optimization steps specified for each plot in the remainder of this section is twenty-five.

The fourth example for this chapter is the conducting sphere. The impulse response of the backscattered field of the sphere is generated with a bandwidth of $k r=0.1(0.1) 33.5$. The duration of the K-pulse response $\left(T_{R}\right)$ and the cutoff time for the integration $\left(T_{C}\right)$ are chosen to be $4 \pi r / c$ and $10 \pi r / c$, respectively. For the $K$-pulse duration $\left(T_{S 1}\right)$ guess of $3 \pi r / c$, the $k$-pulse $\left[k_{s}(t)\right]$ is as shown in Figure 4.19, after 
optimization. The response of the sphere in the backscattered direction is obtained and shown in Figure 4.20. For the K-pulse duration $\left(T_{s 2}\right)$ guess of $4 \pi r / c$, the second guess $k$-pulse $k_{s 2}(t)$ as shown in Figure 4.21 and the sphere response (Figure 4.22 ) to this K-pulse are then produced. Comparing the zeros of $k_{s 1}(t)$ and $k_{s 2}(t)$ (Table 4.6), the first approximation for the duration of the sphere's K-pulse is $2.254 \pi \mathrm{r} / \mathrm{c}$ comparing with the true value of $2 \pi r / c$. The reason that this number is not as good as before is the low 0 property of the sphere. Using $T=2 \pi r / c$, the $K$-pulse with the proper $K$-pulse duration can be obtained as in Figure 3.22. The responses of the sphere at various angle to this K-pulse are shown in Figures 3.23 to 3.27. The poles obtained from the K-pulse confirm the duration of the sphere to be $2 \pi r / c$. Again when the duration of the $K$-pulse $\left(T_{s 3}\right)$ is chosen as $\mathrm{ir} / \mathrm{C}$, the zeros from the "K-pulse" (Figure 4.23) do not resemble those obtained for the sphere previously, even though the $K$-pulse response (Figure 4.24 ) exhibits some energy reduction at late time.

The last example discussed in this chapter is the conducting circular disk. The impulse response whose bandwidth is $k r=0.16(0.16) 16$ at $\theta=45^{\circ}$, and $\hat{\theta}$-polarization is used. The K-pulse response duration $\left(T_{R}\right)$ and the cutoff time $\left(T_{C}\right)$ are chosen to be $4 \pi r / c$ and $8 \pi r / c$, respectively. The first guess $k$-pulse $\left[k_{d l}(t)\right]$ and the response of the circular disk at $\theta=45^{\circ}$, and $\hat{\theta}$-polarization to this $K$-pulse with a K-pulse duration $\left(T_{d 1}\right)$ of $3 \pi r / c$ are shown in figures 4.25 and 4.26 , respectively. When the second guess of the $K$-pulse duration $\left(T_{d 2}\right)$ is chosen to be $4 \pi r / c, K$-pulse $\left[k_{d 2}(t)\right]$ as in Figure 4.27 is 
obtained. The corresponding response of the disc at $\theta=45^{\circ}$, and $\theta$-polatzation to this K-pulse is shown in Figure 4.28. Comparing the zeros of $k_{d 1}(t)$ and $k_{d 2}(t)$ (Table 4.7), the first approximation to the K-pulse duration $(T)$ is $2.176 \pi r / c$, which compares fairly well with the true value of $2 \pi r / c$. Again, hecause the disk is a low 0 structure, the first approximation is not very close. If $T$ is chosen to be $2 \pi r / c$, then the $K$-pulse and the disc's responses to the $K$-pulse are shown as in Figures 3.28 through 3.32. Again the zero string of the K-pulse verifies the proper K-pulse duration. Figures 4.29 and 4.30 show the "K-pulse" and the response of the disc at $\theta=45^{\circ}, \hat{\theta}=$ polarization to the "K-pulse" when the K-pulse duration is chosen to be $\pi r / c$. Although some energy reduction can be seen, the zero string departs totally from the other zero strings. This means the duration of the K-pulse is chosen too small.

This chapter has presented a procedure to obtain the approximate duration of the K-pulse. The method works better with high $Q$ geometries, even when the $K$-pulse response durations are not known very accurately. The procedure works reasonably well with low Q structures if the proper K-pulse response durations are known. The reason for this discrepancy lies in the concept of minimizing energy outside the K-pulse response duration. When the energy content at late time is low already as in the low $Q$ structures, the optimization process will not be able to perform as well as the impulse responses with high energy content at late time. Nevertheless, this is a very good procedure for estimating or checking the proper duration of the K-pulse. 
Table 4.1

Radian Frequency Difference Between Consecutive Poles

Equation (3.2) Wire Loop Sphere Disk

$\begin{array}{llclll}1) & 6.598 & 3.120 & 6.362 & 5.913 & 6.744 \\ 2) & 6.445 & 3.123 & 6.340 & 5.974 & 6.595 \\ 3) & 6.382 & 3.127 & 6.330 & 6.014 & 6.528 \\ 4) & 6.350 & 3.129 & 6.323 & 6.038 & 6.487 \\ 5) & 6.332 & 3.130 & 6.319 & 6.070 & 6.461 \\ 6) & 6.321 & - & 6.316 & 6.082 & 6.441 \\ 7) & 6.313 & - & 6.313 & 6.095 & 6.426 \\ 8) & 6.307 & - & 6.311 & 6.113 & 6.414 \\ 9) & 6.303 & - & 6.310 & 6.120 & 6.404\end{array}$


Table 4.2

Summary of Radian Frequency Differences and Its Corresponding Time

Radian

Frequency

Difference

Approaching

$\frac{\text { Equation }}{(3.2)}$ Wire Loop Sphere Disk

Corresponding

Time

$2 \pi$

$\pi$

$2 \pi$

$2 \pi$

$2 \pi$

First Radian

$L / C$

$2 \mathrm{~L} / \mathrm{C}$

$2 \pi r / c$

$2 \pi r / c$

$2 \pi r / c$

Difference

6. 598

3.120

6. 362

5.913

6.744

Corresponding Time

$0.952 L / C$

$2.014 \mathrm{~L} / \mathrm{C}$

$1.975 \pi r / c$

$2.013 \pi r / c$

$1.863 \pi r / c$ 
Table 4.3

A Comparison of the Zeros Obtained Using Two K-pulses with Different Durations (The Target is a Straight Wire)

\section{K-pulse Estimate $\left[k_{w 1}(t)\right] \quad k$-pulse Estimate $\left[k_{w 2}(t)\right]$}

$$
\text { (Duration }=3 L / C) \quad \text { (Duration }=4 L / C \text { ) }
$$

1) $-0.649+2.099 j$

2)

$$
\begin{aligned}
& -0.102+1.304 j \\
& -0.178+2.967 j \\
& -0.404+4.301 j \\
& -0.260+6.093 j \\
& -0.587+7.475 j \\
& -0.318+9.194 j
\end{aligned}
$$$$
\text { 3) }-0.184+2.977 j
$$$$
\text { 4) }
$$$$
\text { 5) }-0.234+6.105 j
$$

6)

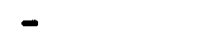

7) $-0.316+9.200 j$ 
Table 4.4

A Comparison of the Zeros Obtained Using Two K-pulses with Different Durations (The Target is a Circular Loop)

$K$-pulse Estimate $\left[k_{\ell 1}(t)\right] \quad K$-pulse Estimate $\left[k_{\ell 2}(t)\right]$

(Duration $=3 \pi r / c) \quad$ (Duration $=4 \pi r / c$ )

1)

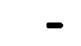

2) $-1.187+3.232 j$

3) $-0.432+6.520 j$

$-0.442+6.510 j$

4)

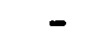

$-1.101+9.438 j$

5) $\quad-0.557+12.859 j$

$-0.634+12.881 j$

6)

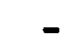

7) $-0.779+19.246 j$

$-1.218+16.044 j$

$-0.789+19.229 j$ 


\section{Table 4.5}

A Comparison of the Zeros Obtained Using Two K-pulses with Different Durations [The Simulated Model in Equation (3.2)]

$$
K \text {-pulse Estimate }\left[k_{e}(t)\right] \quad K \text {-pulse Estimate }\left[k_{e 2}(t)\right]
$$

$$
\text { (Duration }=1.5 \mathrm{~L} / \mathrm{C}) \quad \text { (Duration }=2 \mathrm{~L} / \mathrm{C} \text { ) }
$$

1) $\quad-3.639+5.201 j$

$-3.356+5.380 j$

2) $-5.034+11.830 j$

$-3.860+11.633 j$

3) $-6.197+17.960 j$

$-4.379+18.417 j$

4) $\quad-5.659+24.707 j$

$-4.358+24.659 j$

5) $-6.125+33.393 j$

$-4.310+31.174 j$ 


\section{Table 4.6}

A Comparison of the Zeros Obtained Using Two K-pulses with Different Durations (The Target is a Conducting Sphere)

$$
\begin{aligned}
& \text { K-pulse Estimate }\left[k_{s 1}(t)\right] \quad k \text {-pulse Estimate }\left[k_{s 2}(t)\right] \\
& \text { (Duration }=3 \pi r / c) \quad \text { (Duration }=4 \pi r / c \text { ) }
\end{aligned}
$$

1)

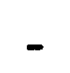

$$
-1.478+4.036 j
$$

2) $-2.998+5.287 j$

$-3.405+5.419 j$

3) $-4.700+10.862 j$

$-2.047+10.414 j$

4) $-3.518+16.428 j$

$-2.291+16.359 j$

5) $-4.197+22.252 j$

$-2.510+22.313 j$ 


\section{Table 4.7}

A Comparison of the Zeros Obtained Using Two K-pulses with Different Durations (The Target is a Circular Disk)

$K$-pulse Estimate $\left[k_{d 1}(t)\right] \quad K$-pulse Estimate $\left[k_{d 2}(t)\right]$

$$
\text { (Duration }=3 \pi r / c) \quad \text { (Duration }=4 \pi r / c \text { ) }
$$

1)

$$
-0.808+3.180 j
$$

2) $-1.522+4.082 j$

3) $\quad-3.040+7.793 j$

$-3.048+7.411 j$

4)

$$
-
$$$$
-2.219+8.513 j
$$

5) $-3.662+13.566 \mathrm{~J}$

$-2.222+13.697 j$ 


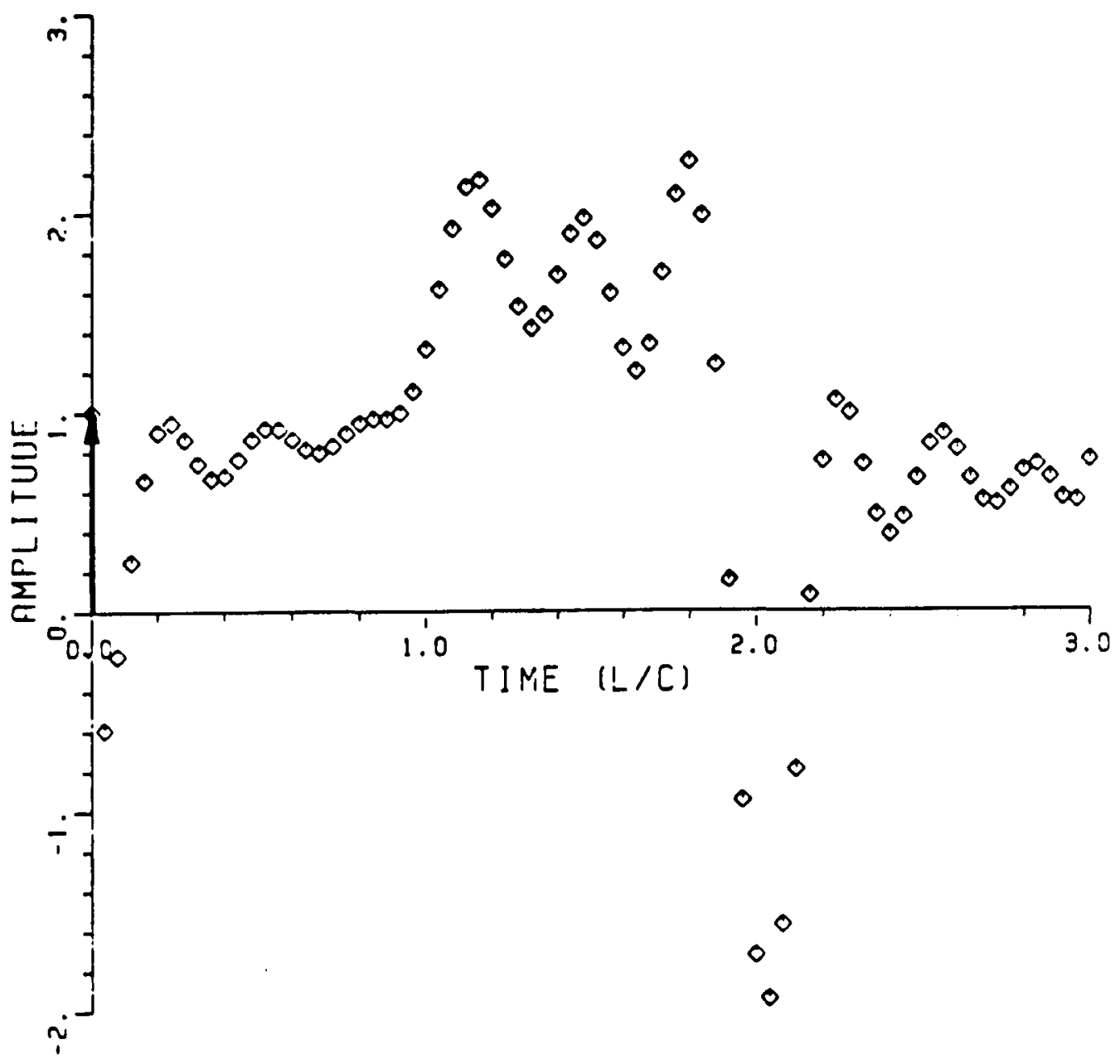

Figure 4.1. K-pulse obtained using the Fourier synthesized impulse response of the conducting straight wire at $\phi=30^{\circ}$ with $K L=0.08 \pi(0.08 \pi) 8 \pi$. The $K$-pulse duration is $3 L / c$. 


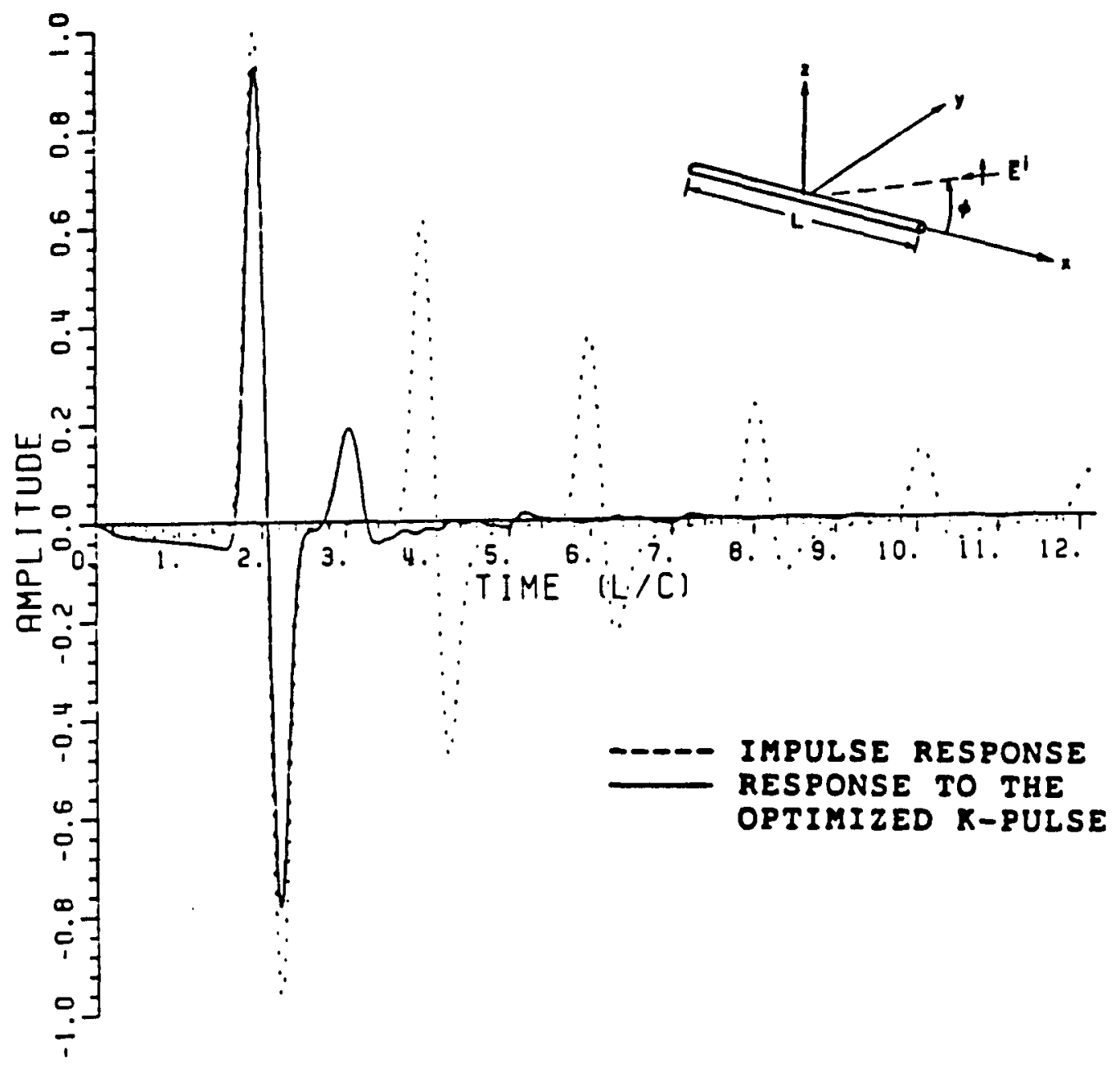

Figure 4.2. Impulse response and response to the $K$-pulse shown in Figure 4.1 for the straight wire at $\phi=30^{\circ}$ with $K L=0.08 \pi(0.08 \pi) 8 \pi$. 


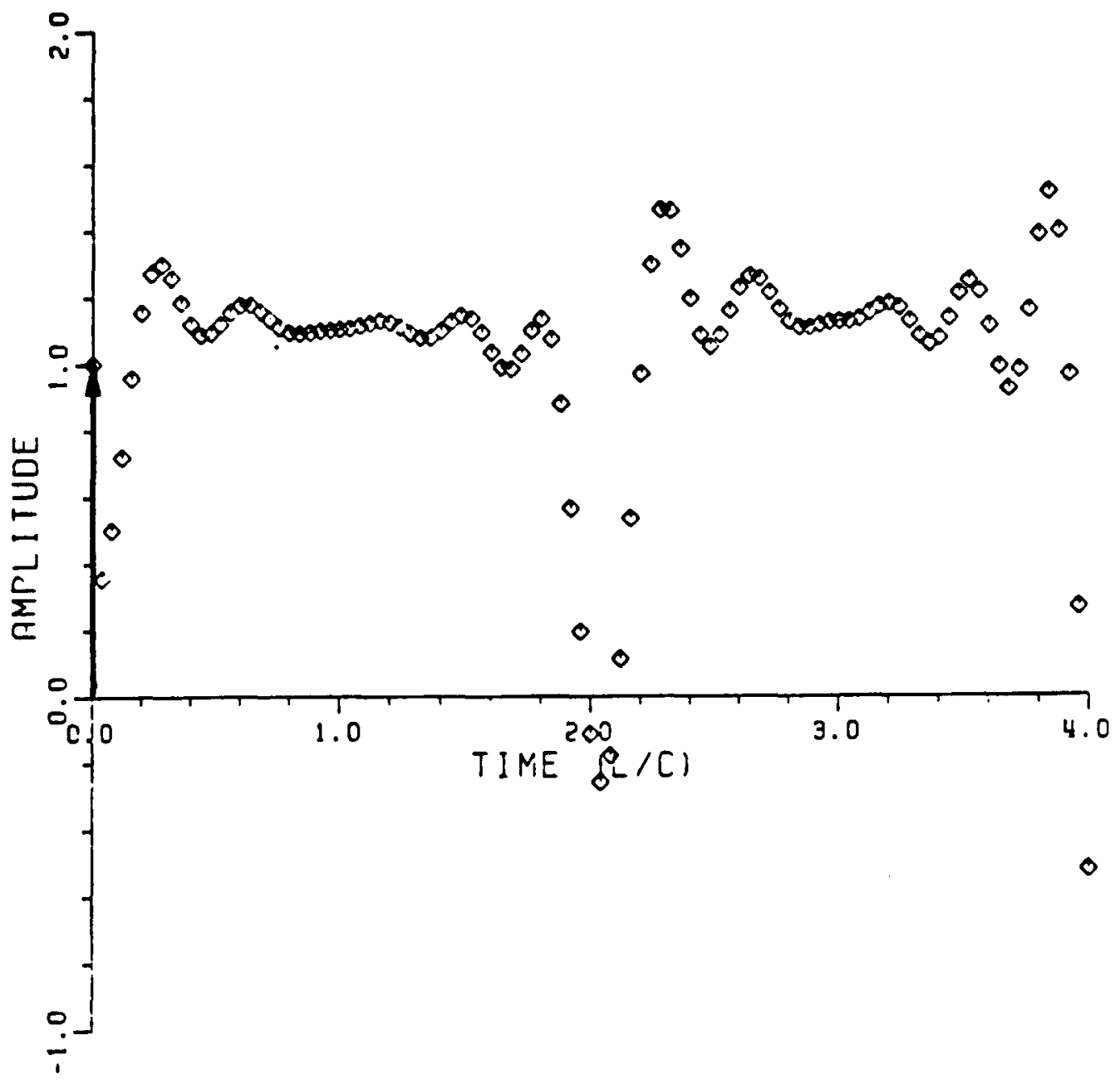

Figure 4.3. K-pulse obtained using the Fourier synthesized impulse response of the conducting straight wire at $\phi=30^{\circ}$ with $K L=0.08 \pi(0.08 \pi) 8 \pi$. The K-pulse duration is $4 L / C$. 


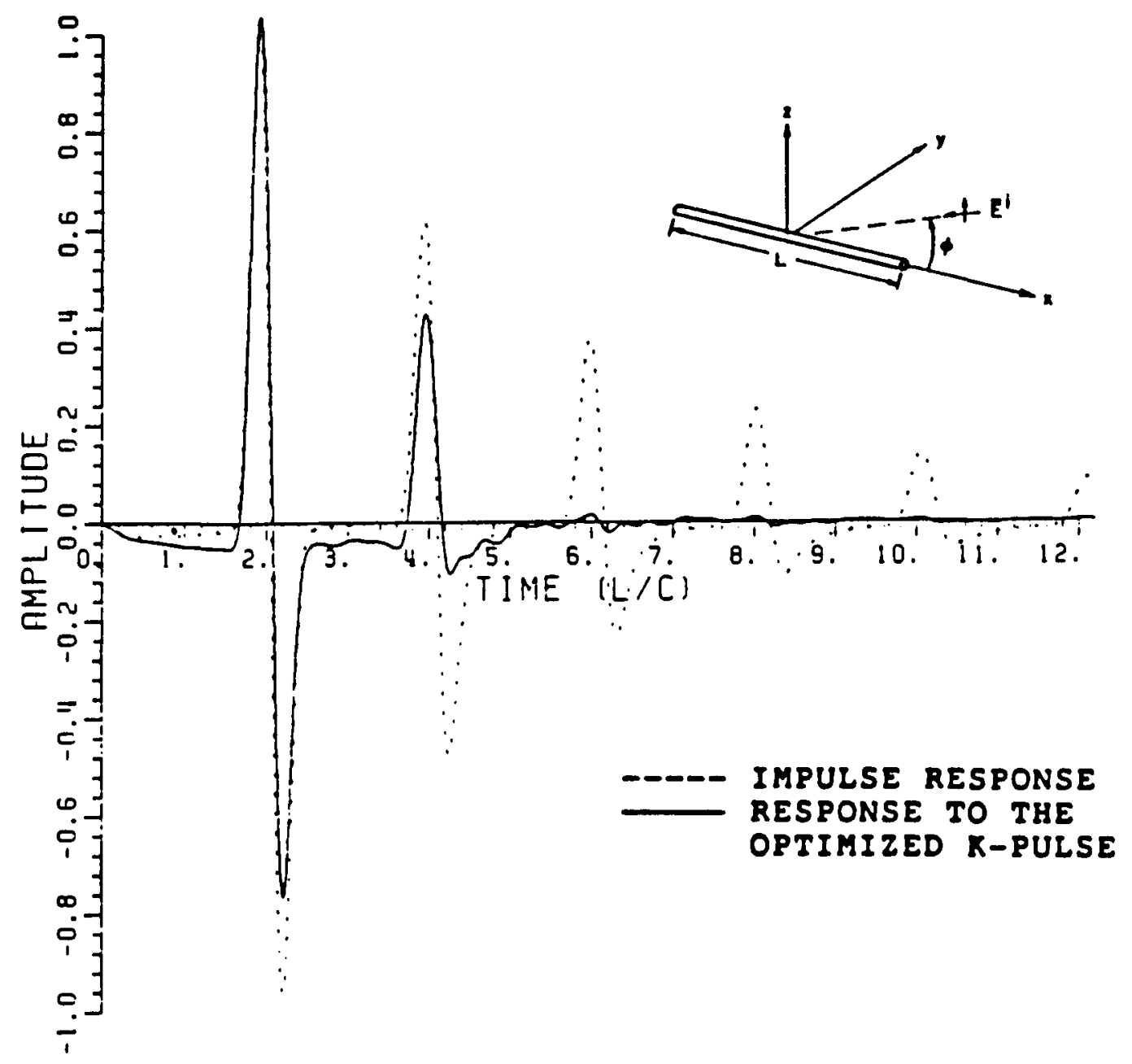

Figure 4.4. Impulse response and response to the $K$-pulse shown in Figure 4.3 for the straight wire at $\phi=30^{\circ}$ with $K L=0.08 \pi(0.08 \pi) 8 \pi$. 


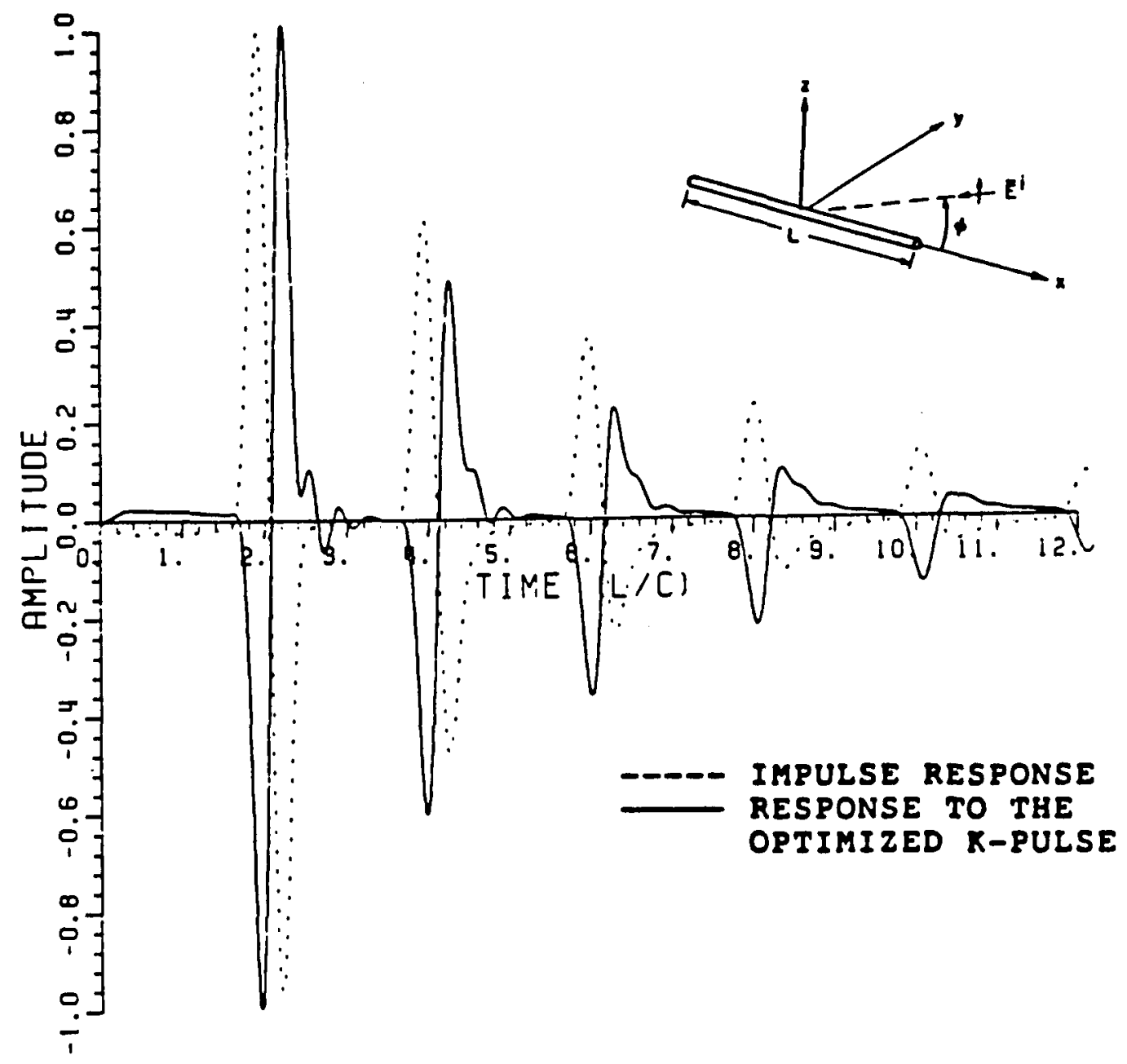

Figure 4.5. Impulse response and response to the "K-pulse" shown in Figure 4.6 for the straight wire at $\phi=30^{\circ}$ with $K L=0.08 \pi(0.08 \pi) 8 \pi$. 


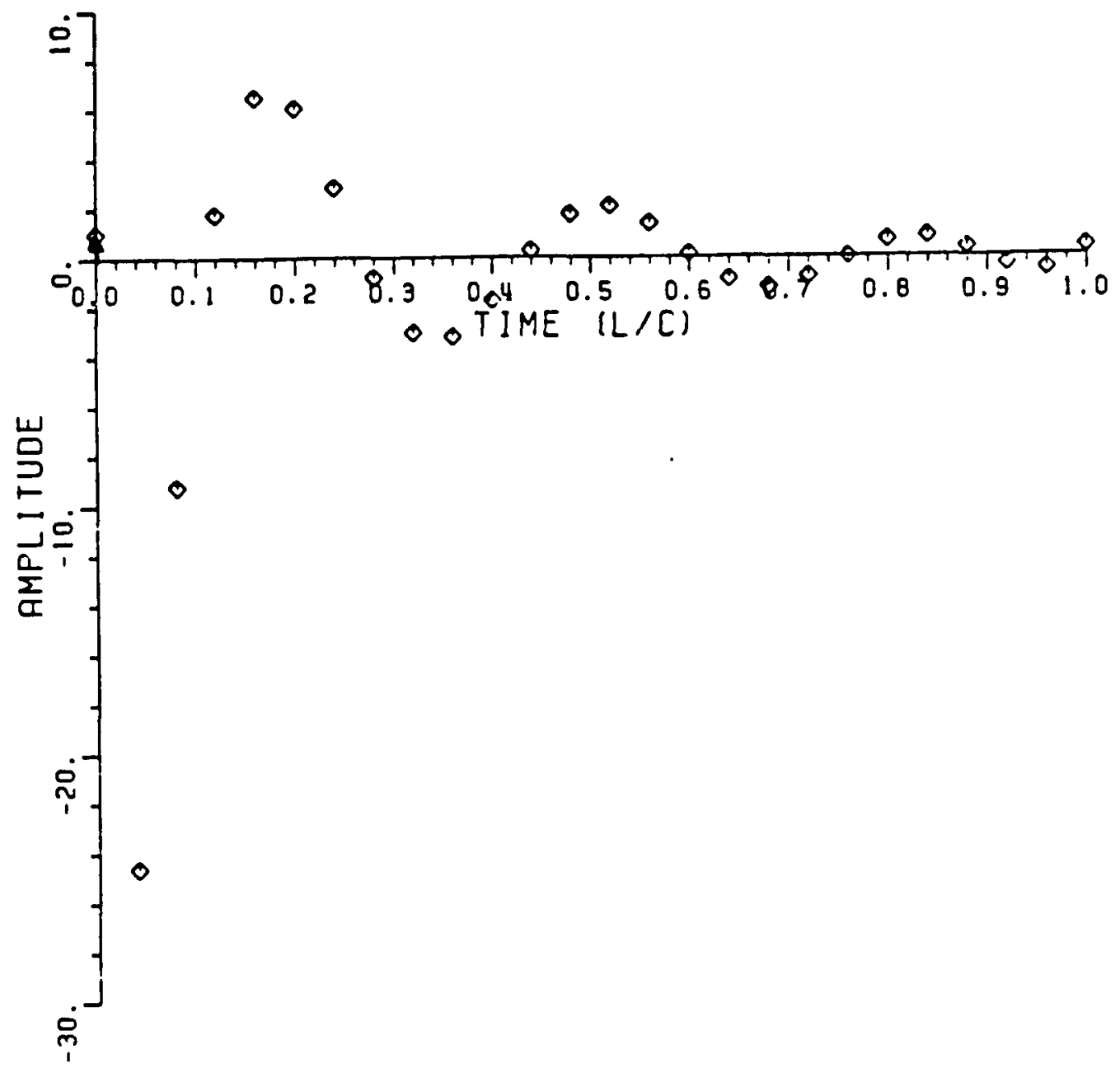

Figure 4.6. "K-pulse" obtained using the Fourier synthesized impulse response of the conducting straight wire at $\phi=30^{\circ}$ with $K L=0.08 \pi(0.08 \pi) 8 \pi$. The "K-pulse" duration is $L / C$. 


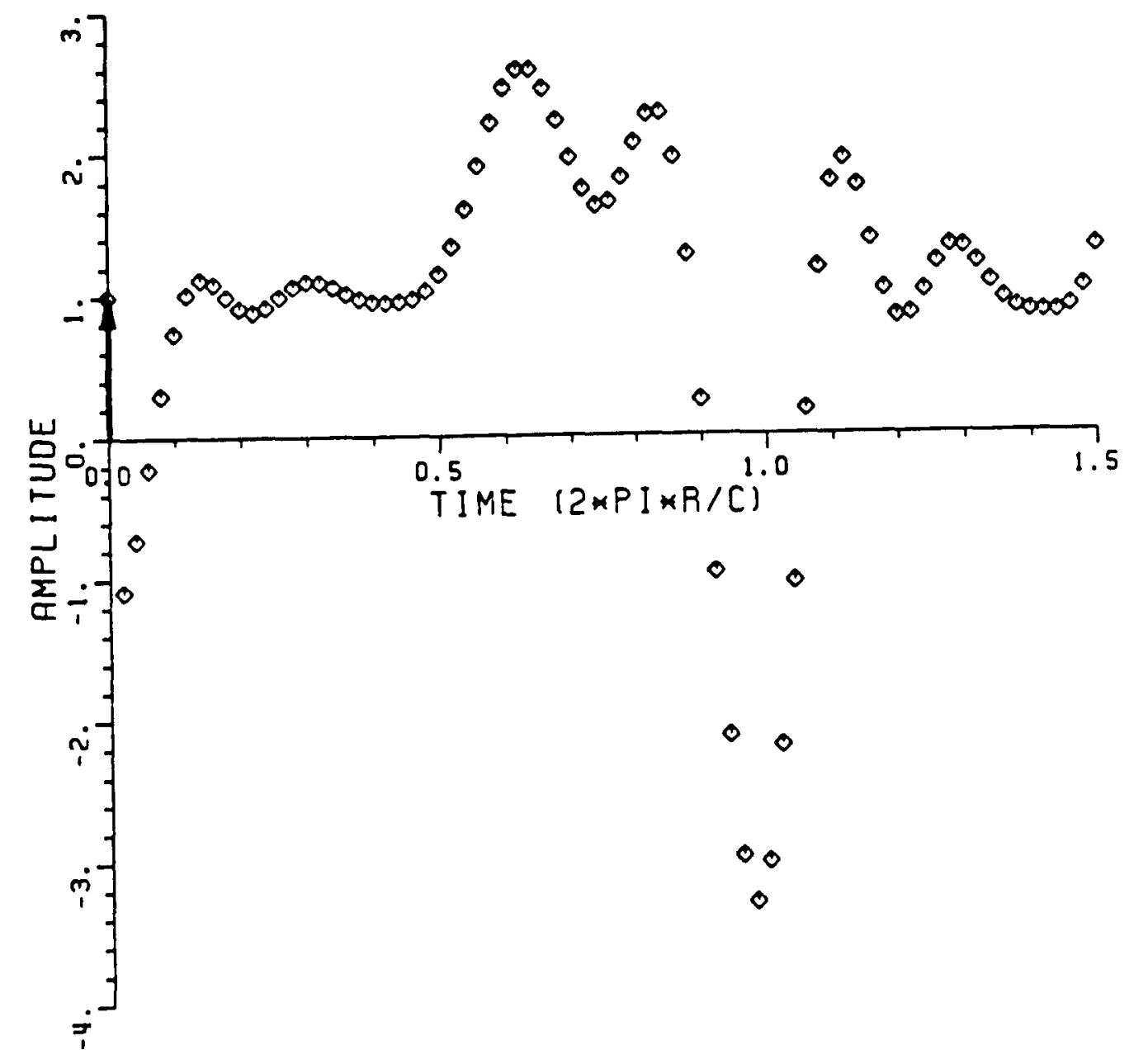

Figure 4.7. K-pulse obtained using the Fourier synthesized impulse response of the conducting circular loop at $\theta=45^{\circ}$ and $\theta$-polarization with $k r=0.02 \pi(0.02 \pi) 4 \pi$. The K-pulse duration is $3 \pi r / c$. 


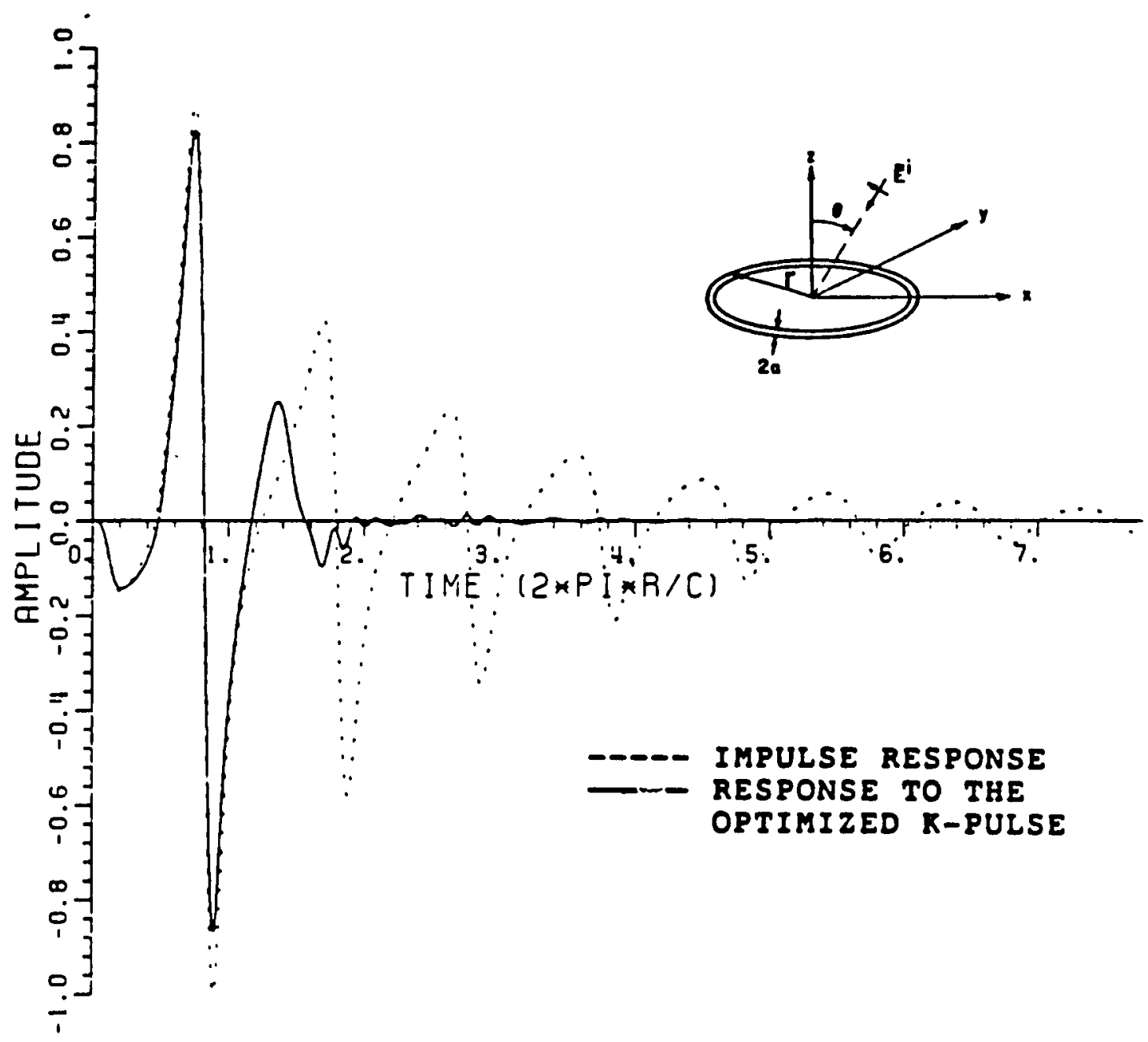

Figure 4.8. Impulse response and response to the $K$-pulse shown in Figure 4.7 for the circular loop at $\theta=45^{\circ}$ and $\hat{\theta}$-polarization with $\mathrm{kr}=0.02 \pi(0.02 \pi) 4 \pi$. 


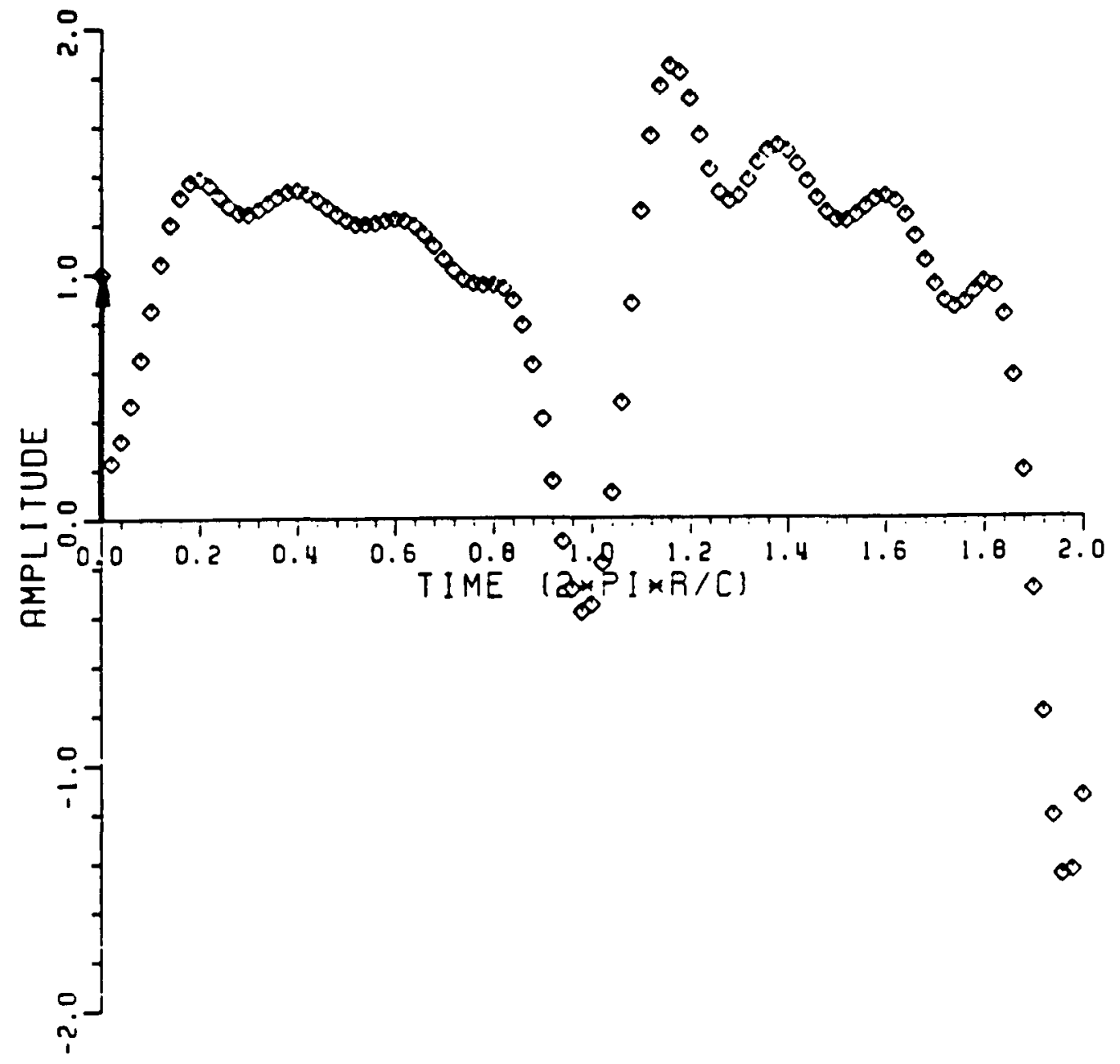

Figure 4.9. K-pulse obtained using the Fourier synthesized impulse response of the conducting circular loop at $\theta=45^{\circ}$ and $\hat{\theta}$-polarization with $k r=0.02 \pi(0.02 \pi) 4 \pi$. The $k$-pulse duration is $4 \pi r / c$. 


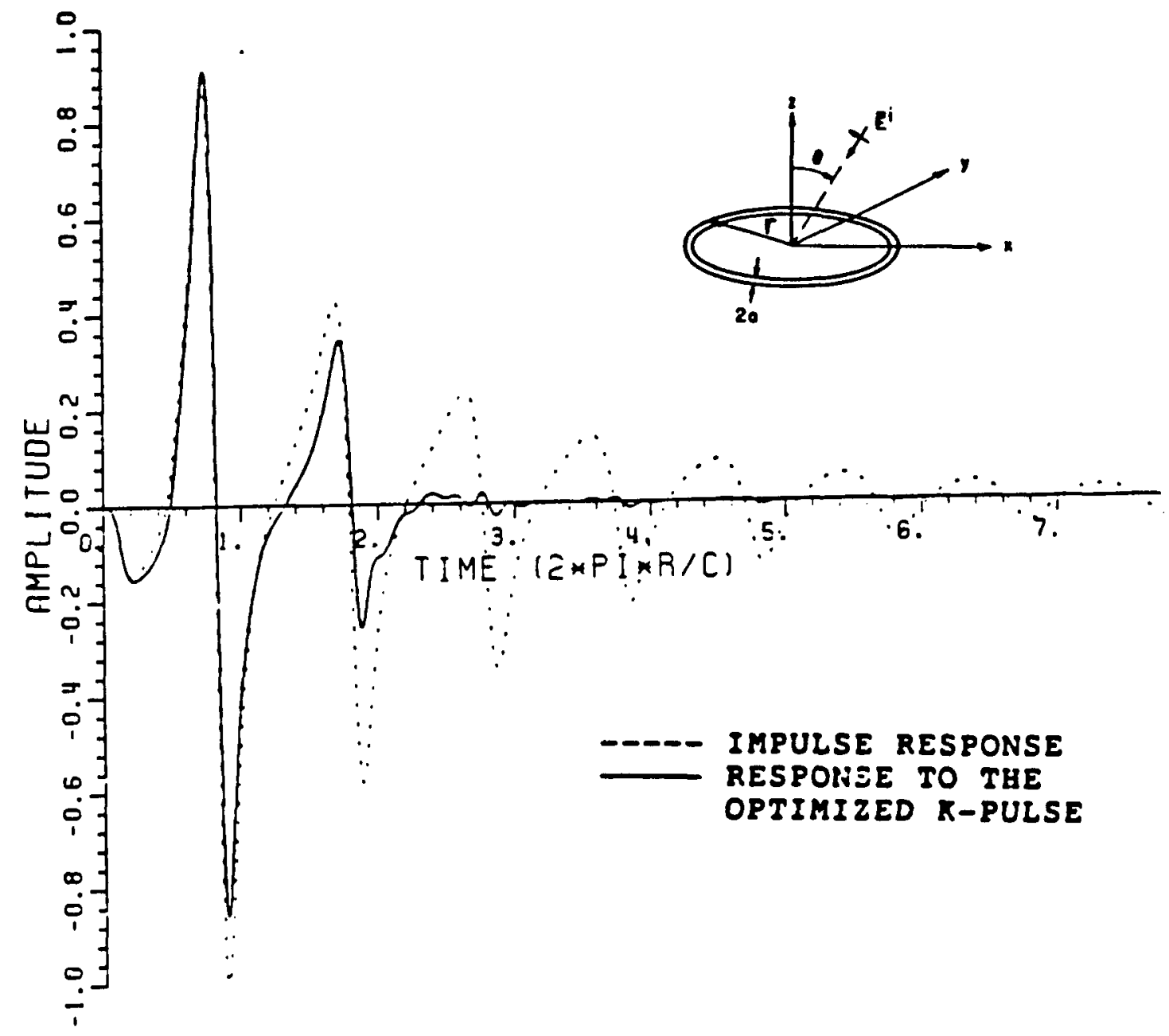

Figure 4.10. Impulse response and response to the $K$-pulse shown in Figure 4.9 for the circular loop at $\theta=45^{\circ}$ and $\hat{\theta}$-polarization with $\mathrm{kr}=0.02 \pi(0.02 \pi) 4 \pi$. 


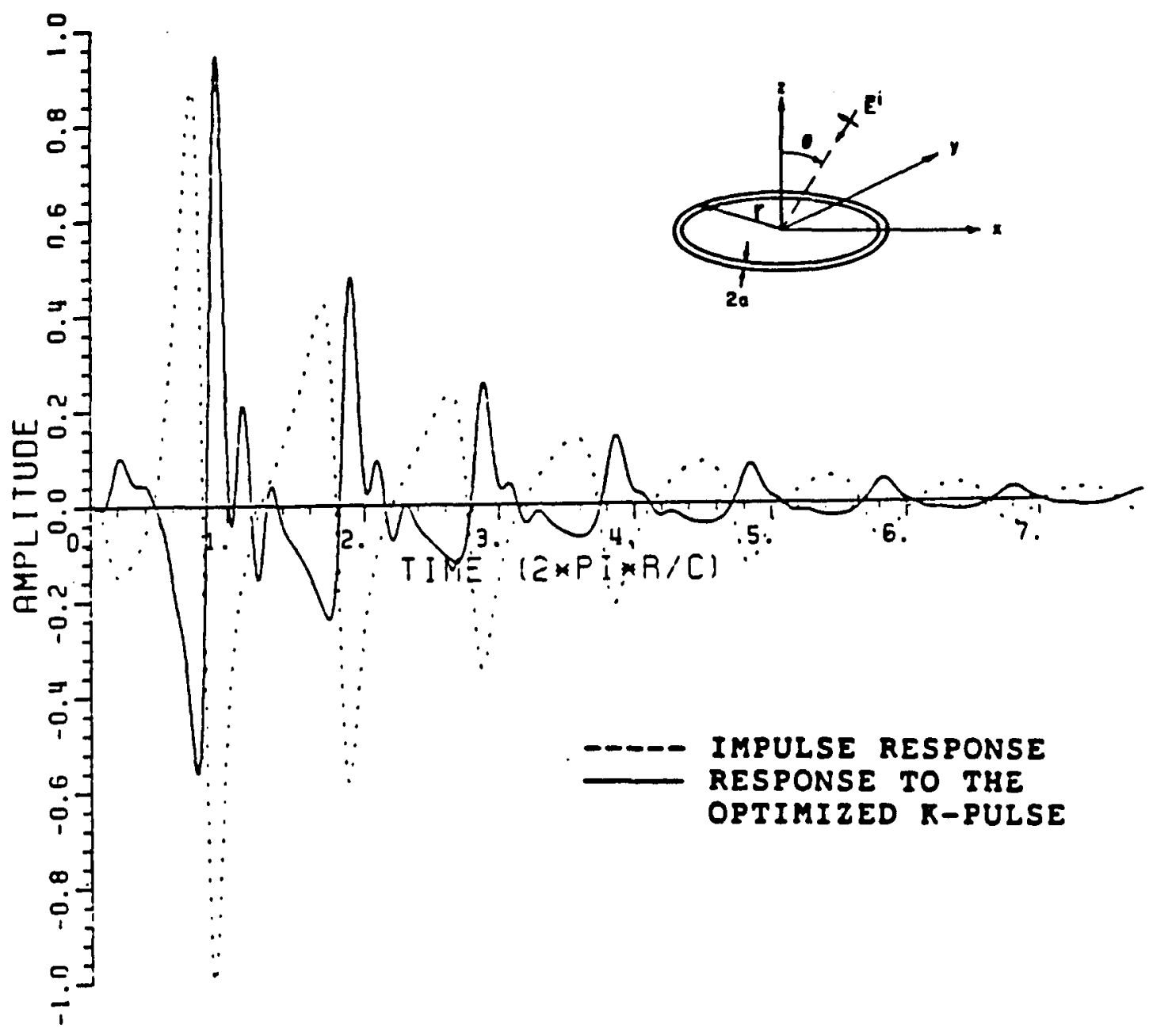

Figure 4.11. Impulse response and response to the "K-pulse" shown in Figure 4.12 for the circular loop at $\theta=45^{\circ}$ and $\hat{\theta}$-polarization with $\mathrm{kr}=0.02 \pi(0.02 \pi) 4 \pi$. 


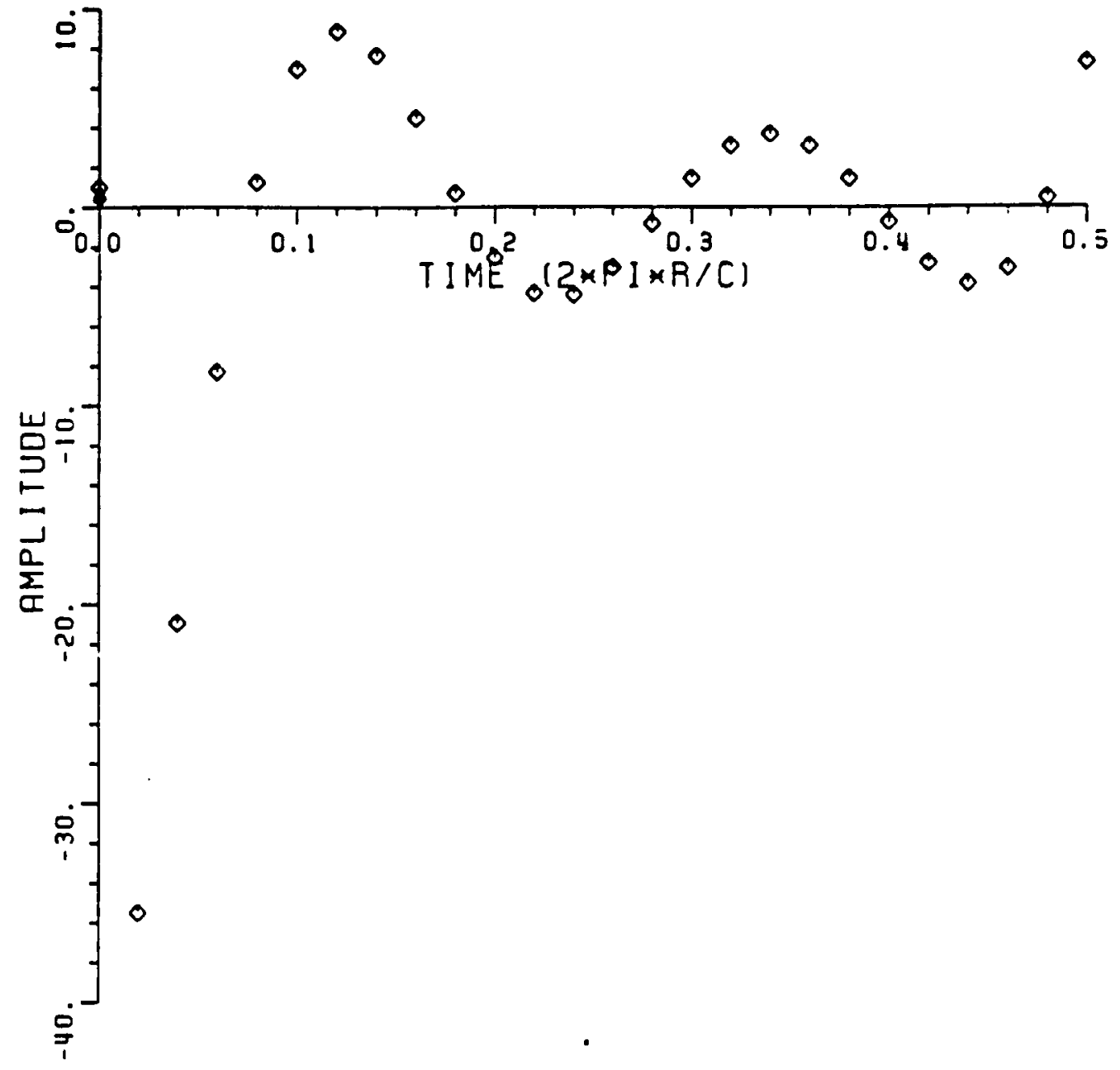

Figure 4.12. "K-pulse" obtained using the Fourier synthesized impulse response of the conducting circular loop at $\theta=45^{\circ}$ and $\hat{\theta}$-polarization with $\mathrm{kr}=0.02 \pi(0.02 \pi) 4 \pi$. The "K-pulse" duration is $\pi r / c$. 


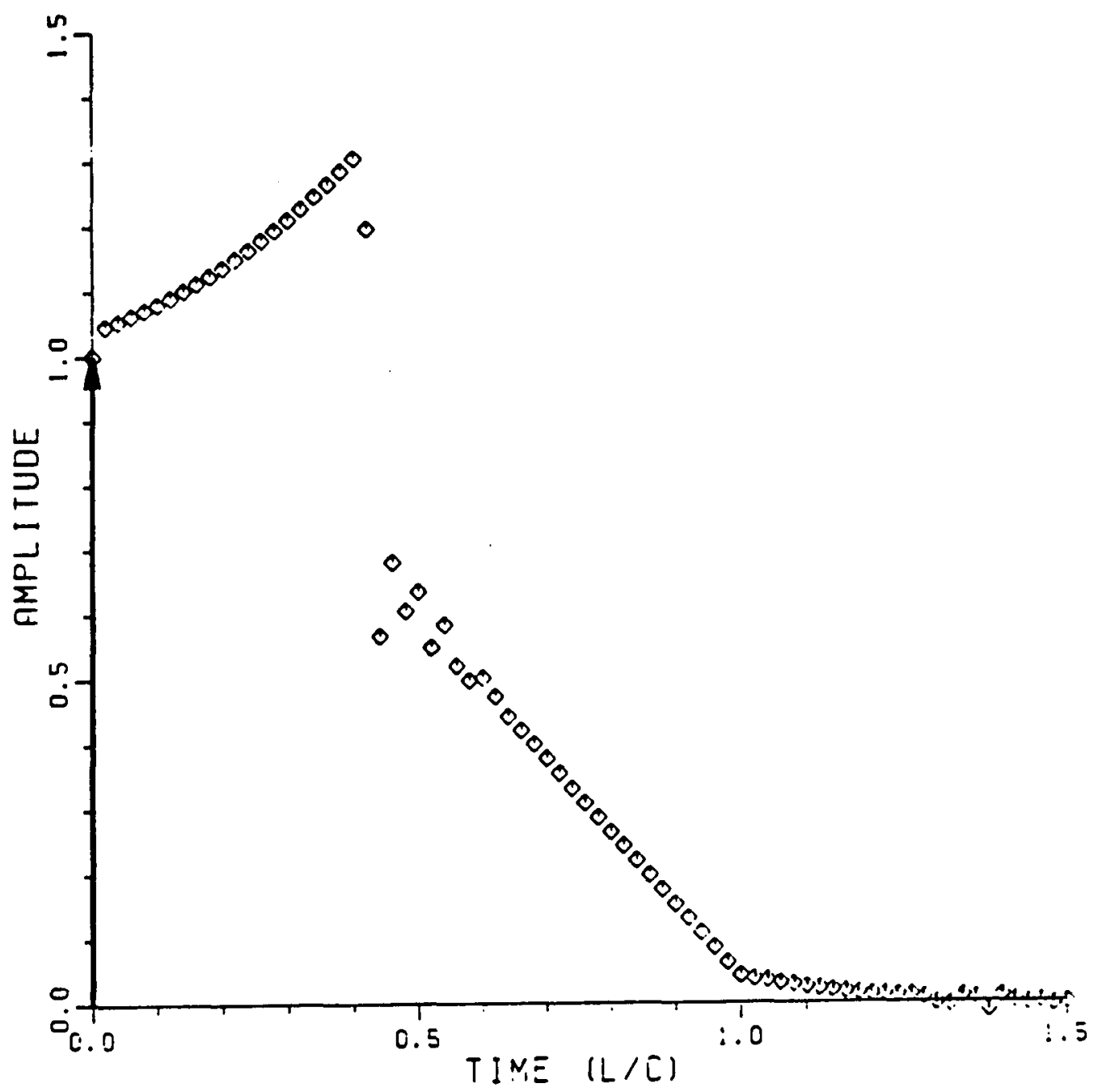

Figure 4.13. K-pulse obtained using the Fourier synthesized impulse response of the simulated model given in Equation (3.2). The K-pulse duration is $1.5 \mathrm{~L} / \mathrm{C}$. 


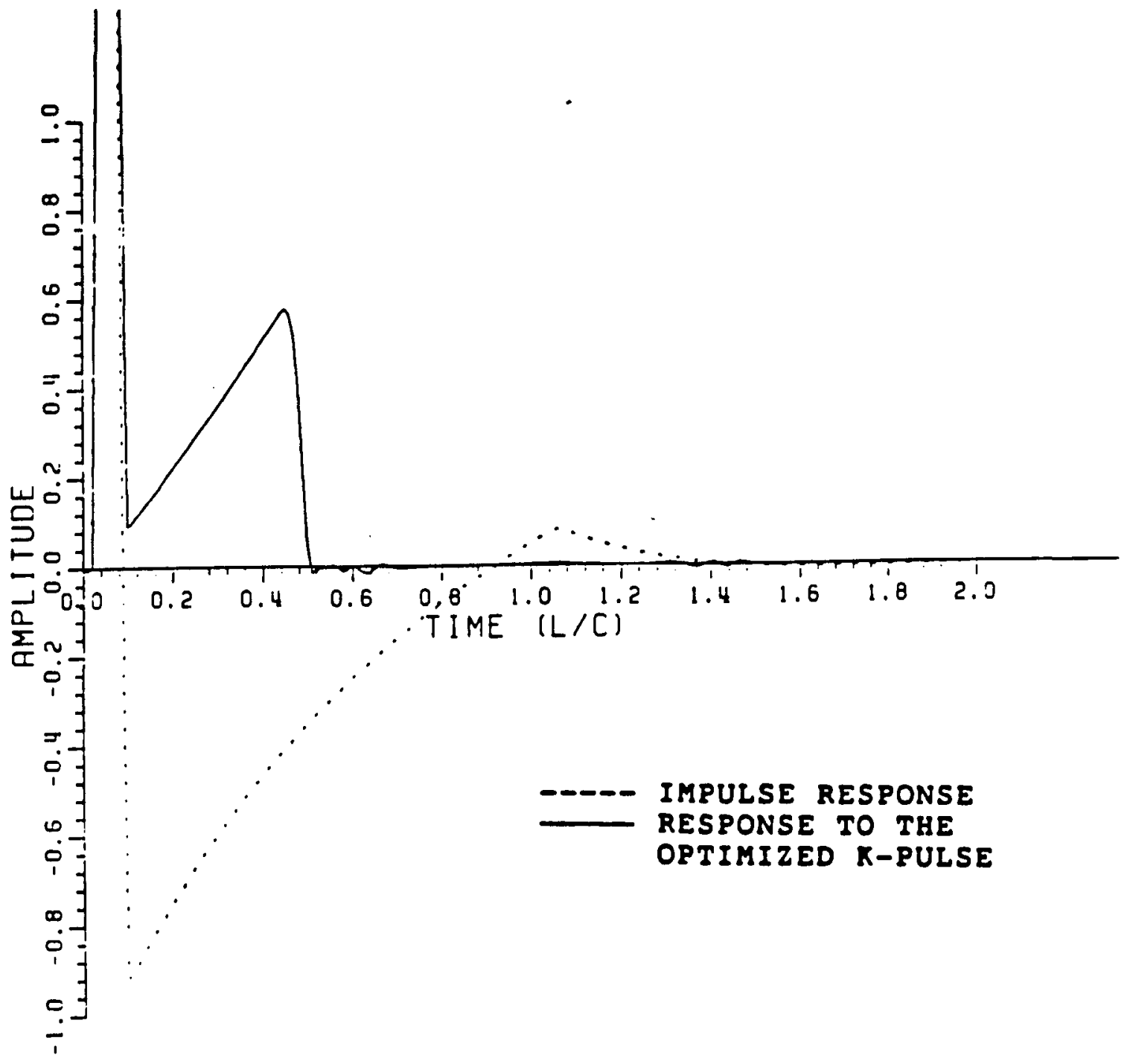

Figure 4.14. Impulse response and response to the $K$-pulse shown in Figure 4.13 for the simulated model given in Equation (3.2). $\quad(K L=0.05 \pi(0.05 \pi) 100 \pi)$. 


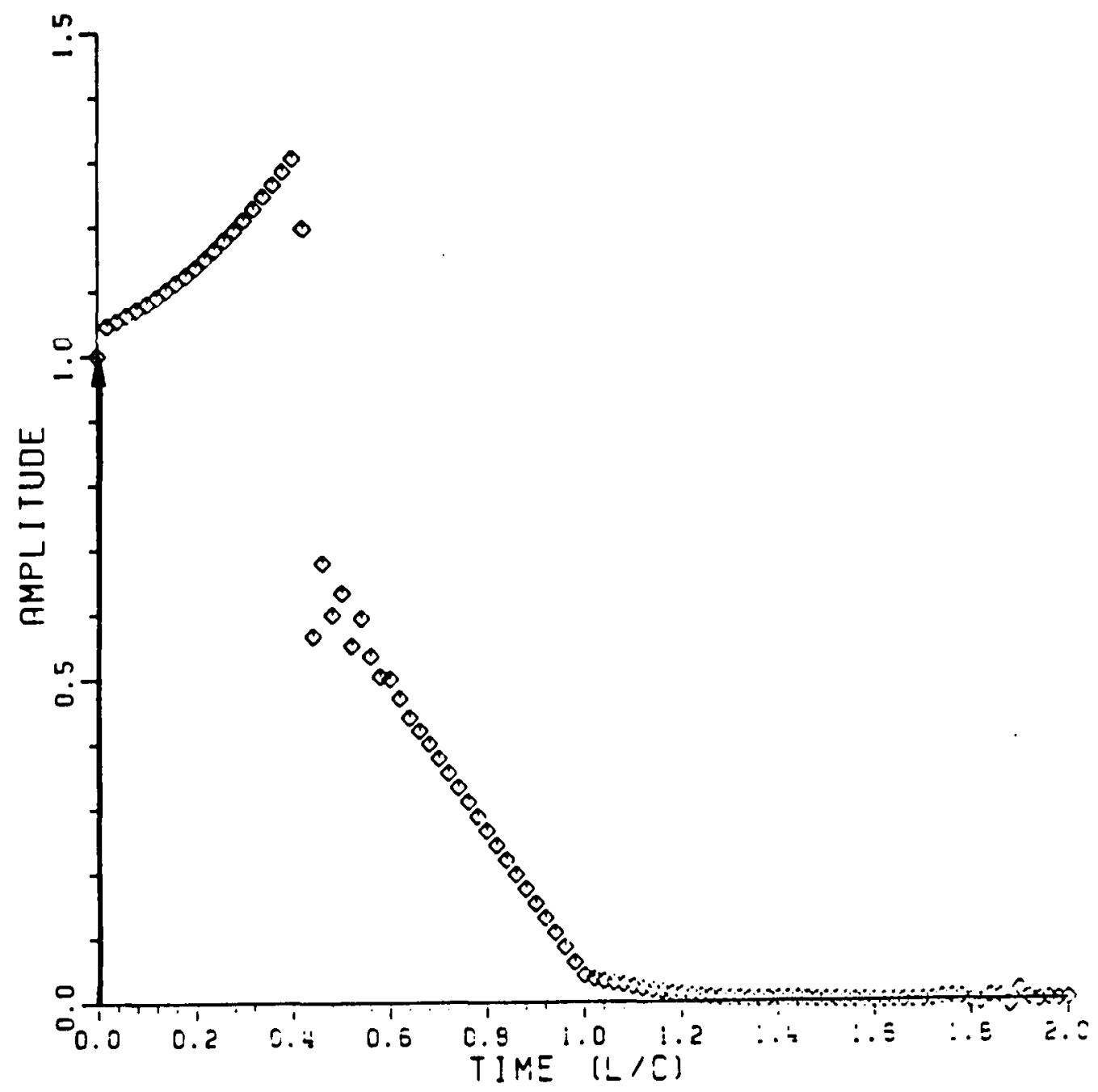

Figure 4.15. K-pulse obtained using the Fourier synthesized impulse response of the simulated model given in Equation (3.2). The K-pulse duration is $2 \mathrm{~L} / \mathrm{C}$. 


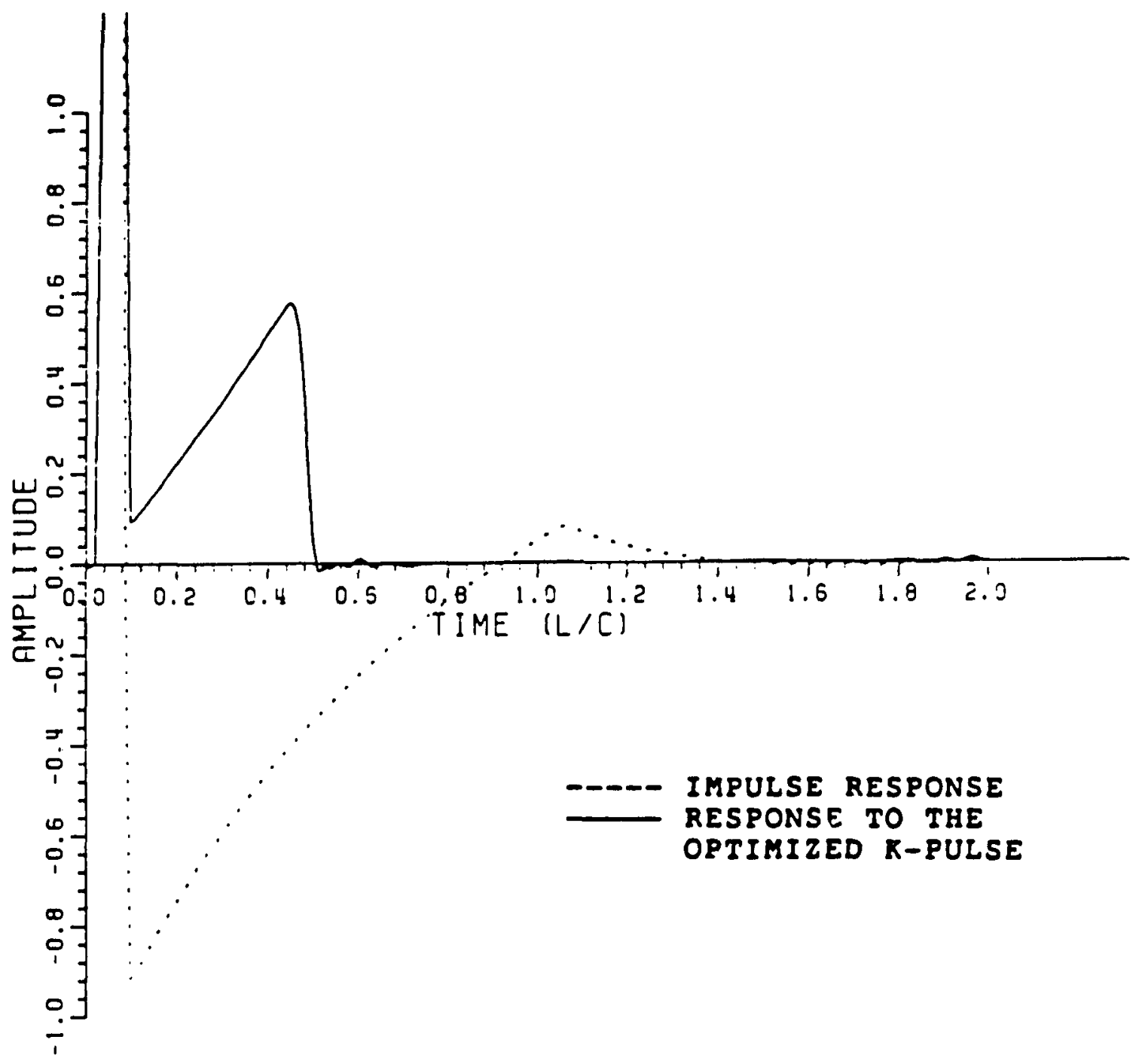

Figure 4.16. Impulse response and response to the $K$-pulse shown in Figure 4.15 for the simulated model given in Equation (3.2). $(k L=0.05 \pi(0.05 \pi) 100 \pi)$. 


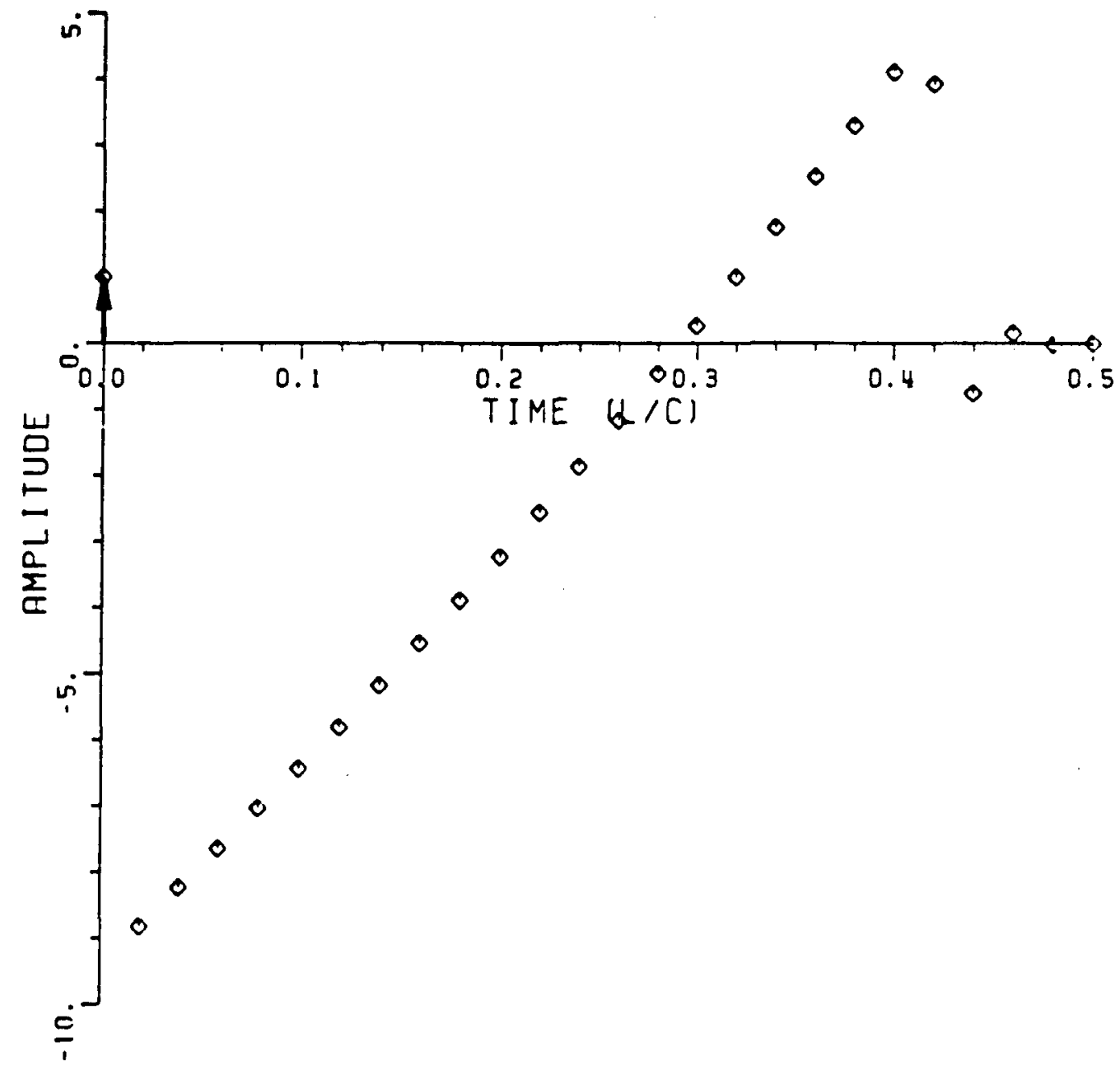

Figure 4.17. "K-pulse" obtained using the Fourier synthesized impulse response of the simulated model given in Equation (3.2). The "K-pulse" duration is $0.5 \mathrm{~L} / \mathrm{C}$. 


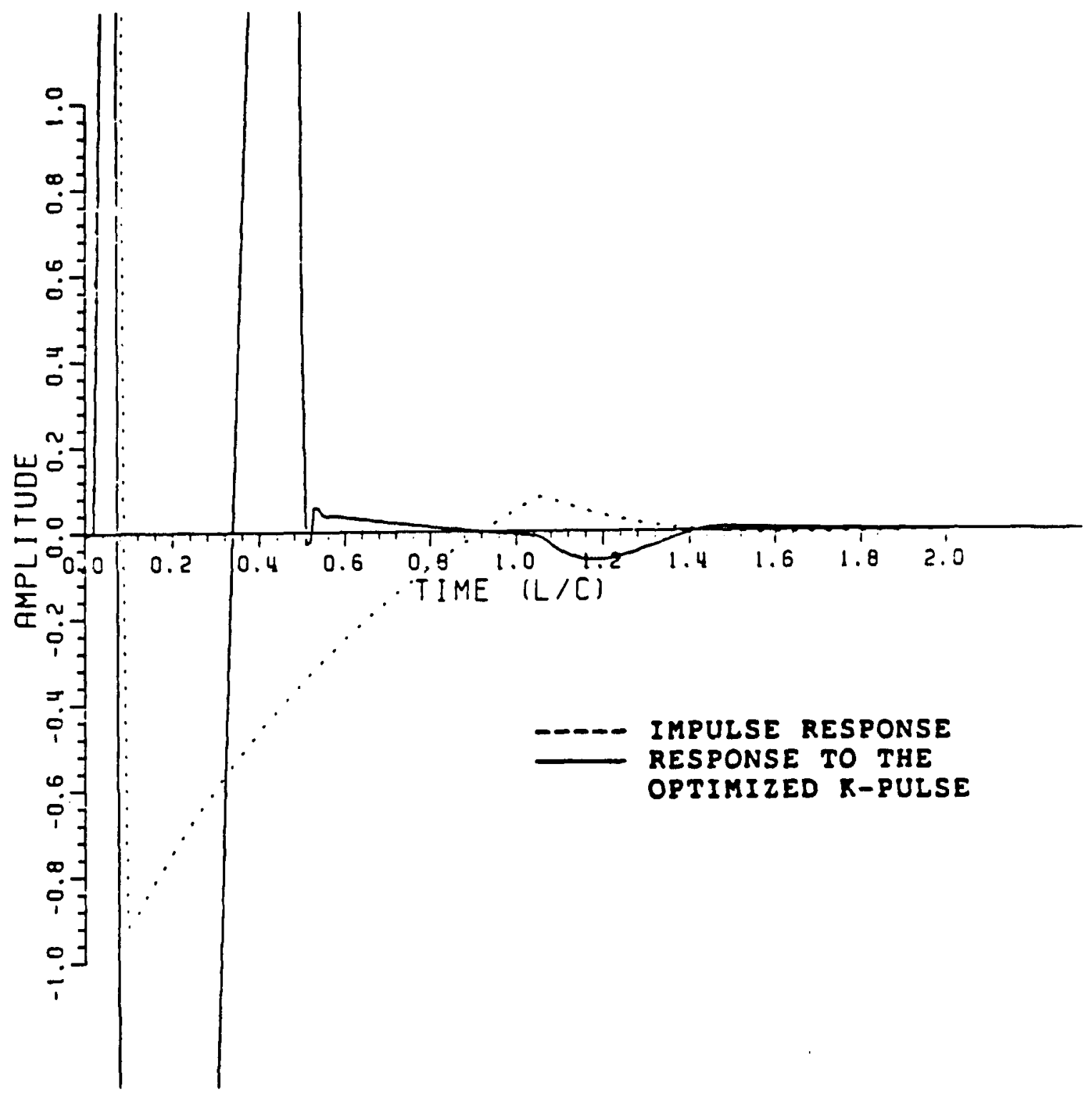

Figure 4.18. Impulse response and response to the "K-pulse" shown in Figure 4.17 for the simulated model given in Equation (3.2). $\quad(k L=0.05 \pi(0.05 \pi) 100 \pi)$. 


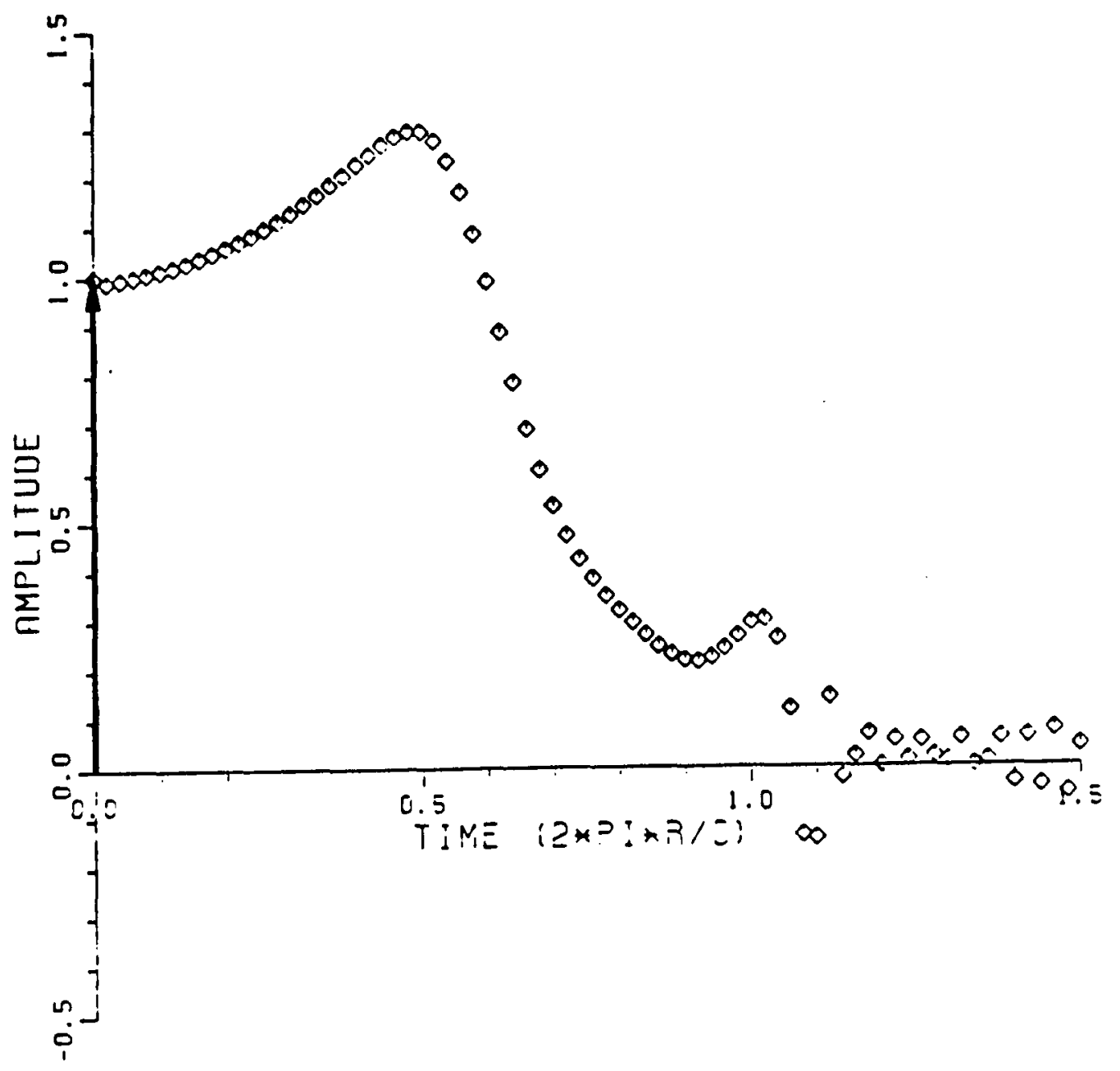

Figure 4.19. K-pulse obtained using the Fourier synthesized impulse response of the conducting sphere in the the backscattered direction with $\mathrm{kr}=0.1(0.1) 33.5$. The K-pulse duration is $3 \pi r / c$. 


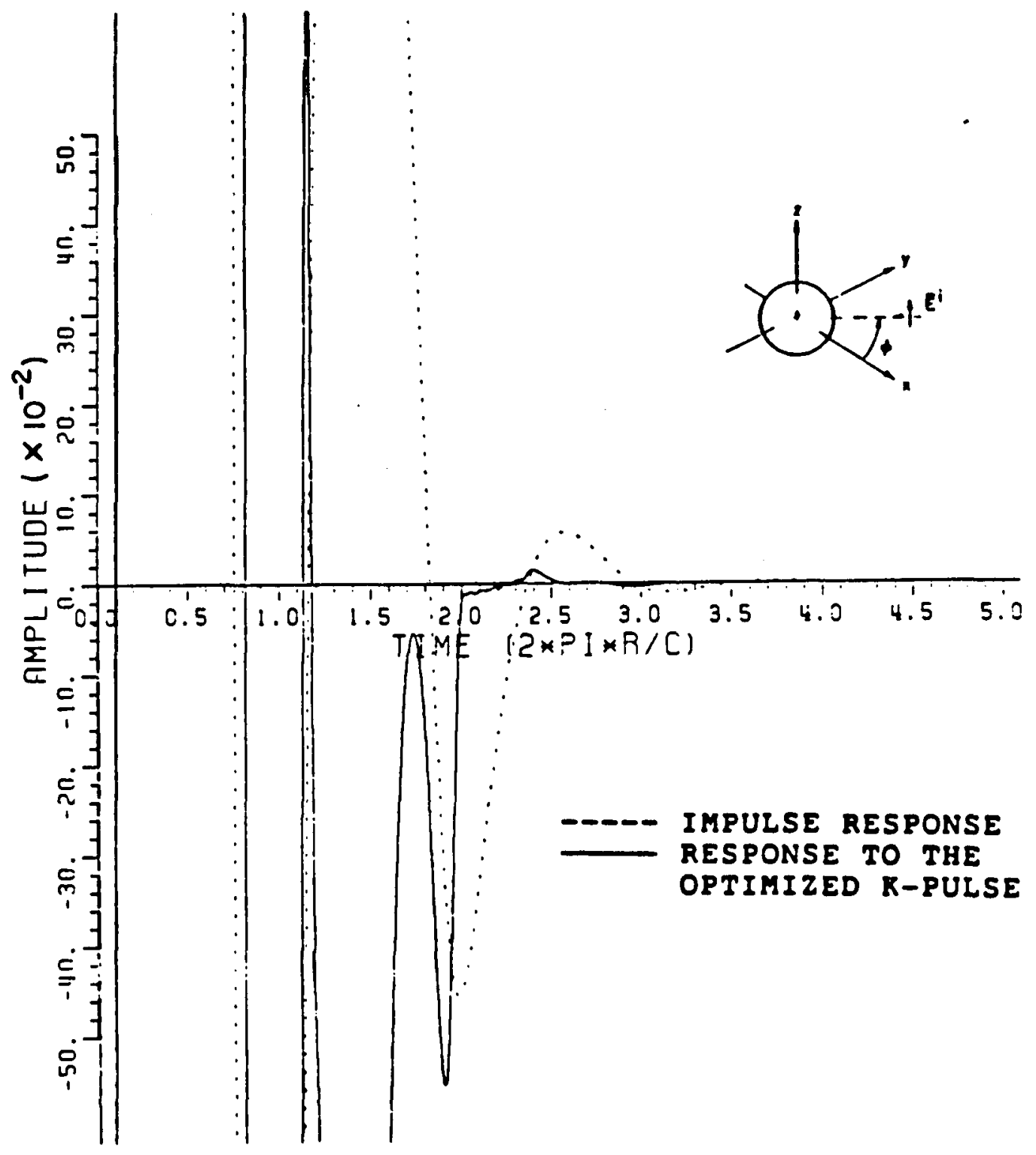

Figure 4.20. Impulse response and response to the $K$-pulse shown in Figure 4.19 for the conducting sphere in the backscattered direction with $\mathrm{kr}=0.1(0.1) 33.5$. The amplitude scale is expanded by 100 . 


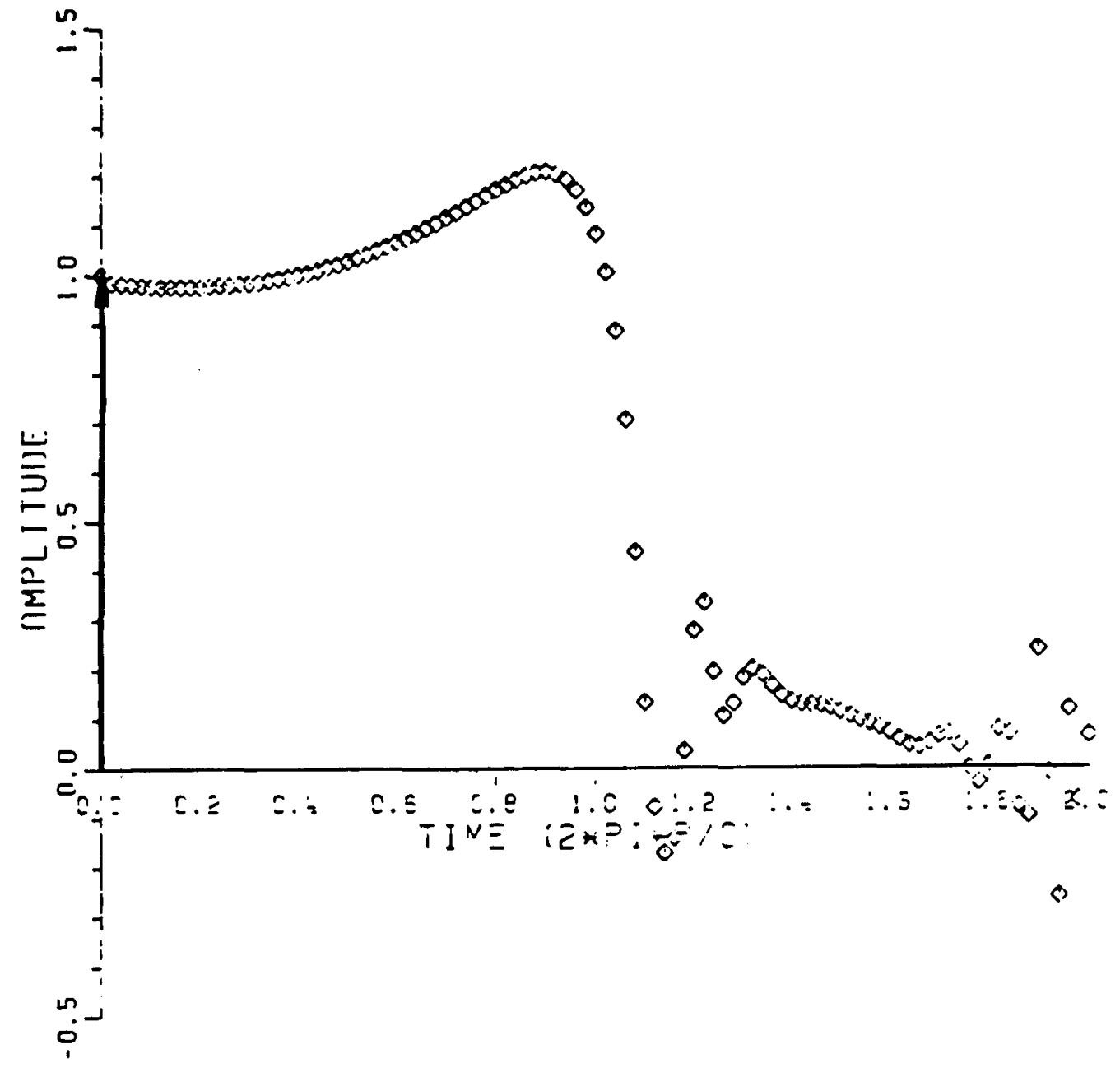

Figure 4.21. K-pulse obtained using the Fourter synthesized impulse response of the conducting sphere in the backscattered direction with $\mathrm{kr}=0.1(0.1) 33.5$. The $\mathrm{K}$-pulse duration is $4 \pi r / c$. 


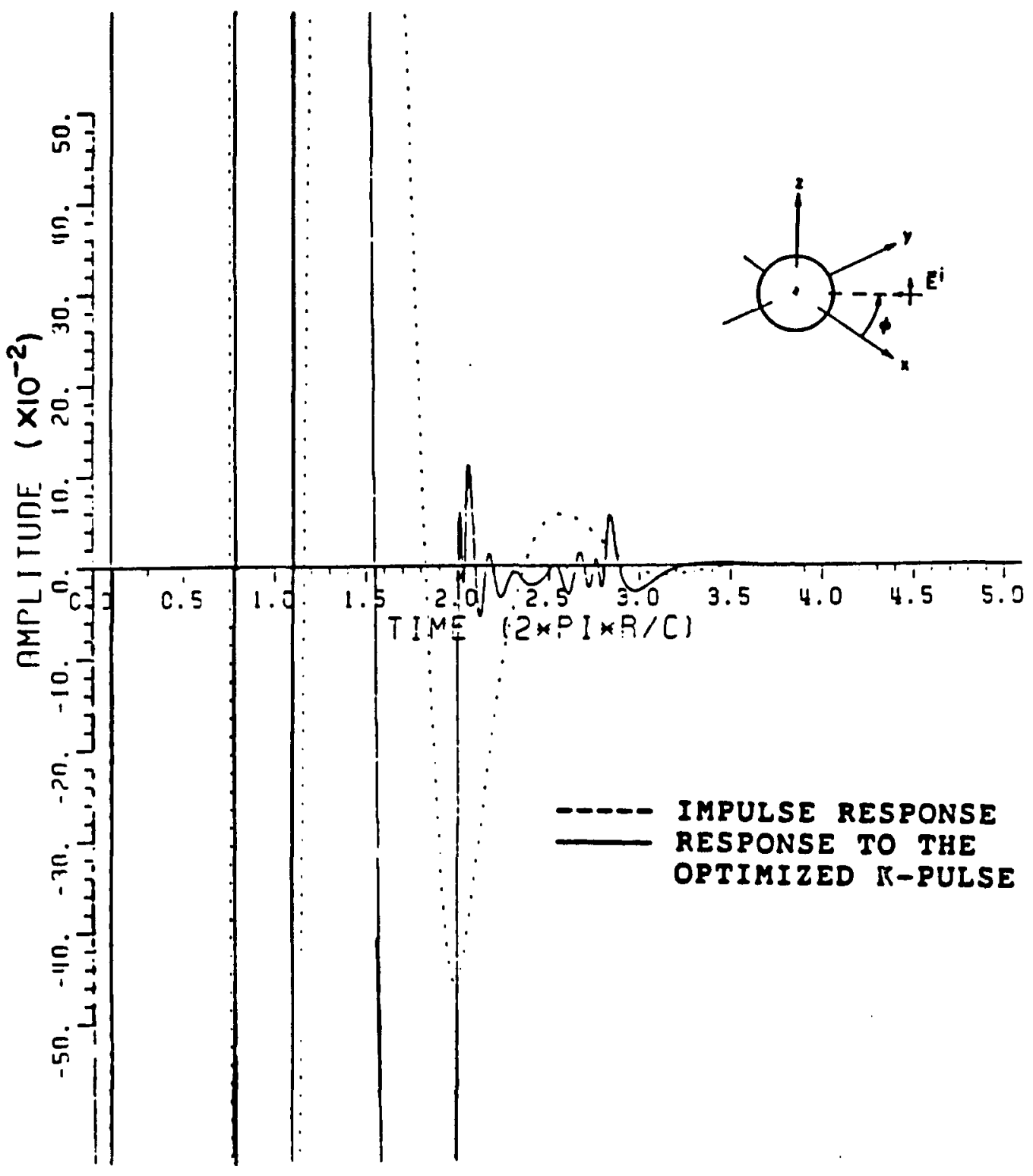

Figure 4.22. Impulse response and response to the $K$-pulse shown in Figure 4.21 for the conducting sphere in the backscattered direction with $\mathrm{kr}=0.1(0.1) 33.5$. The amplitude scale is expanded by 100 . 


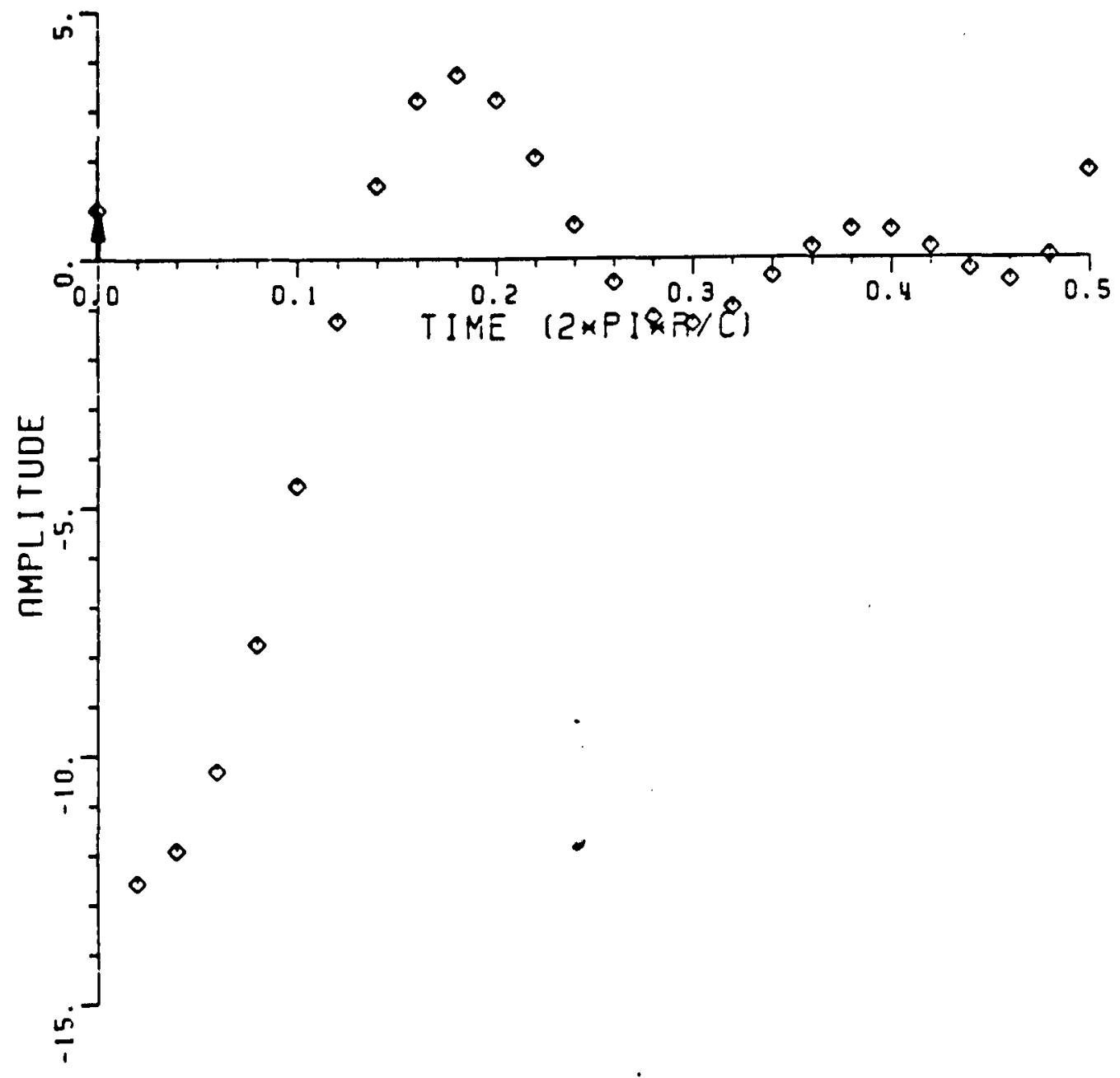

Figure 4.23. "K-pulse" obtained using the Fourier synthesized impulse response of the conducting sphere in the backscattered direction with $k r=0.1(0.1) 33.5$. The "K-pulse" duration is $\pi r / c$. 


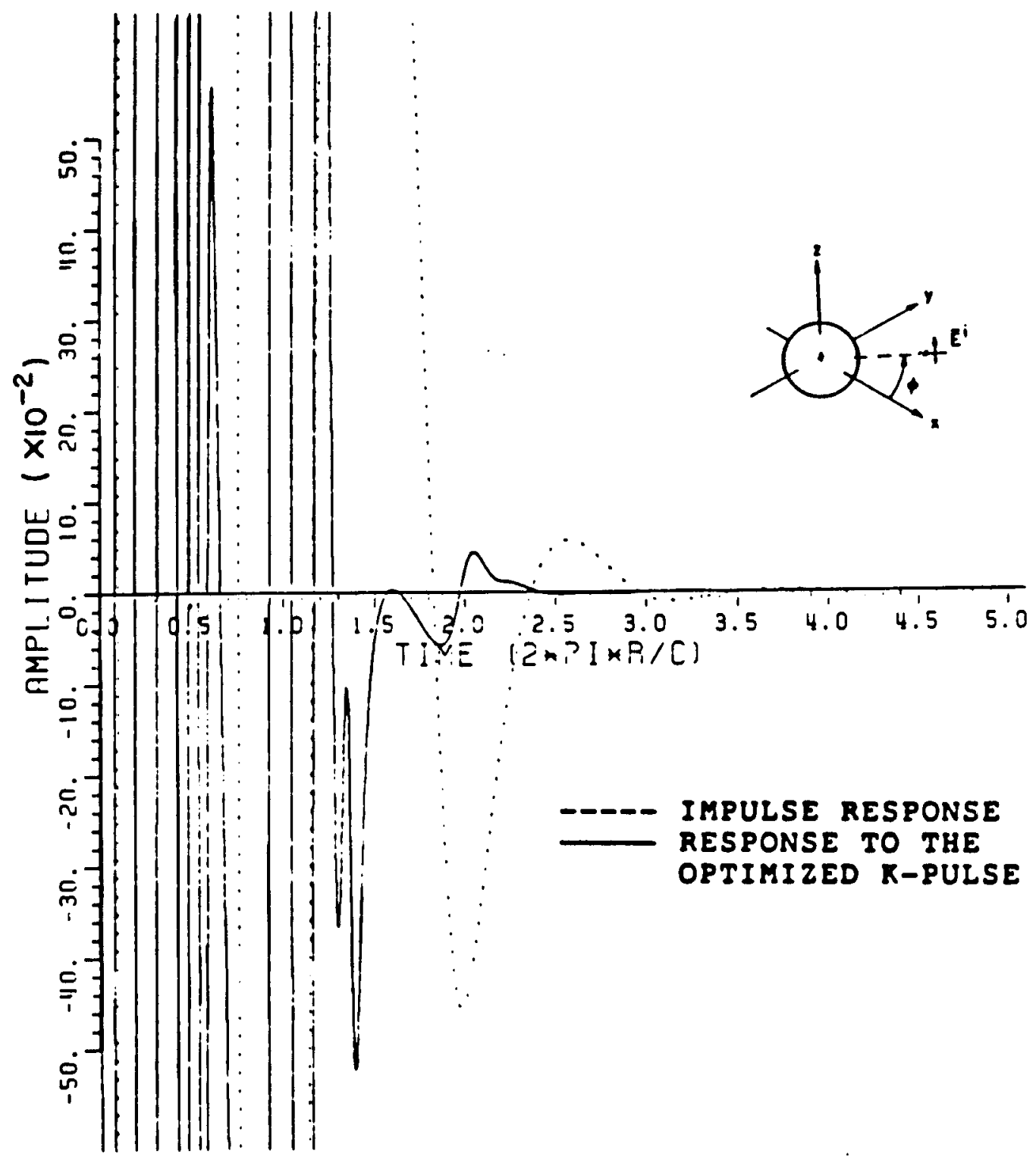

Figure 4.24. Impulse response and response to the "K-pulse" shown in Figure 4.23 for the conducting sphere in the backscattered direction with $k r=0.1(0.1) 33.5$. The amplitude scale is expanded by 100 . 


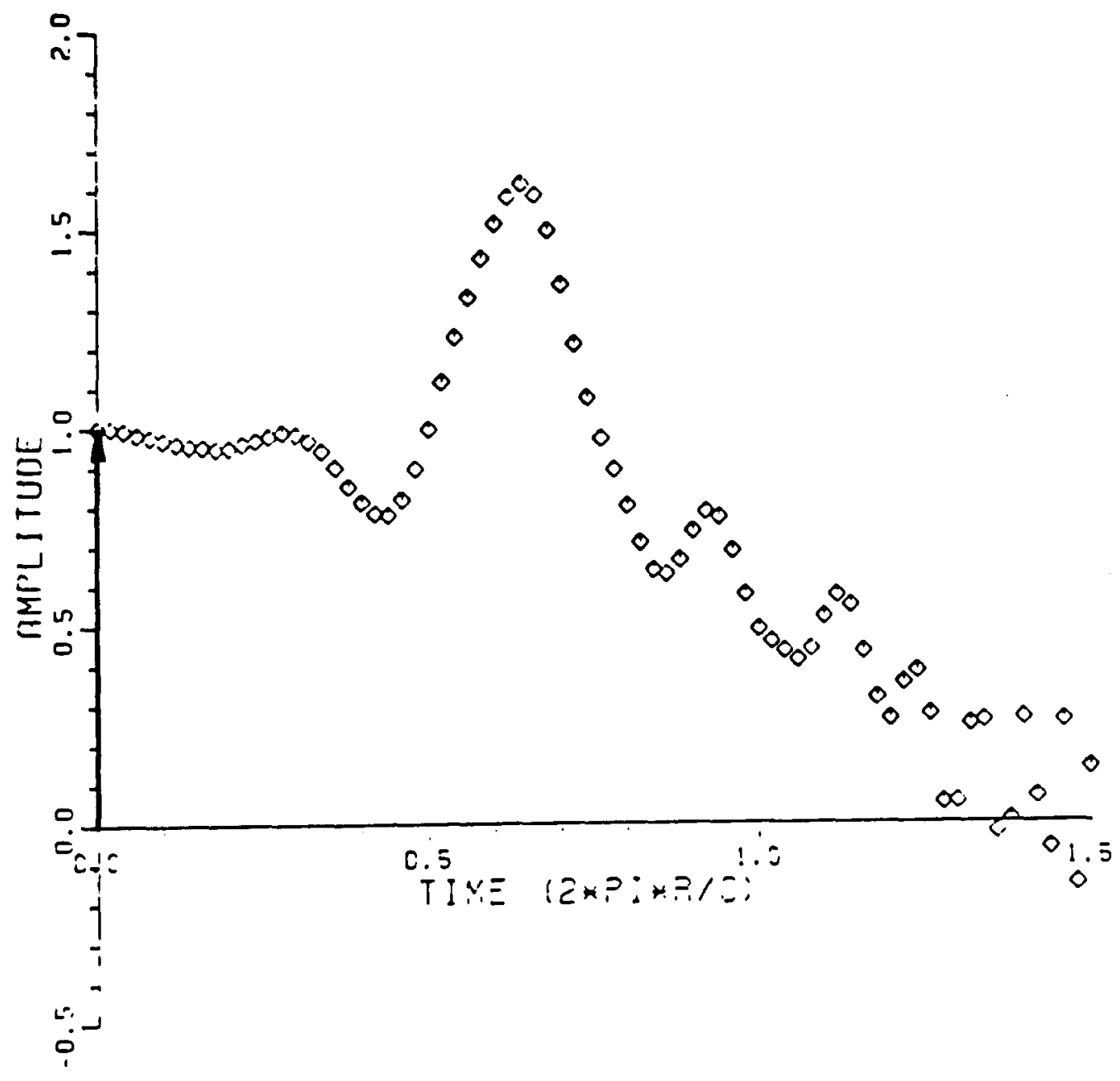

Figure 4.25. K-pulse obtained using the Fourier synthesized impulse response of the conducting circular disk at $\theta=45^{\circ}$, $\hat{\theta}$-polarization with $k r=0.16(0.16) 16$. The $K$-pulse duration is $3 \pi r / c$. 


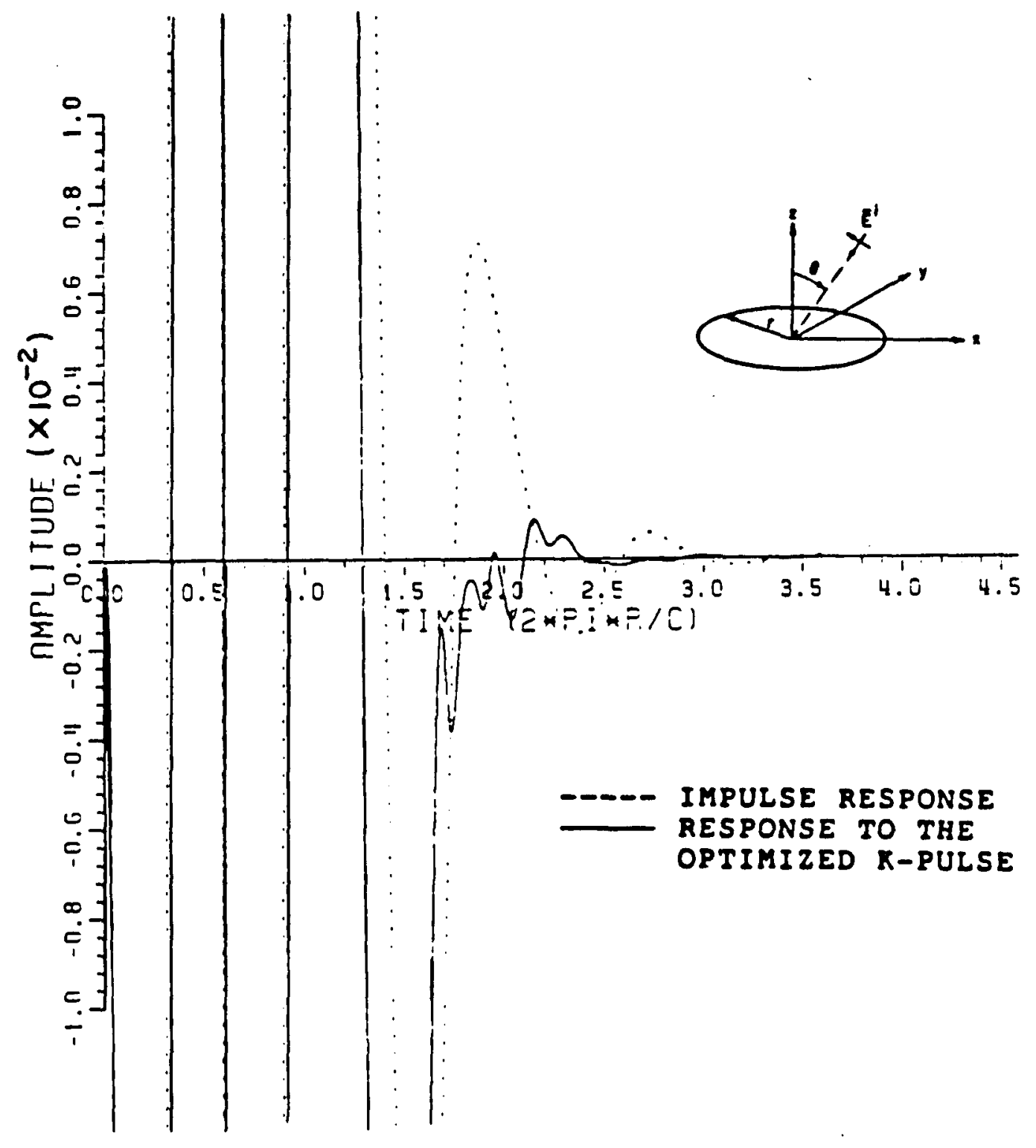

Figure 4.26. Impulse response and response to the $K$-pulse shown in Figure 4.25 for the conducting circular disk at $\theta=45^{\circ}$, $\hat{\theta}$-polarization with $k r=0.16(0.16) 16$. The amplitude scale is expanded by 100 . 


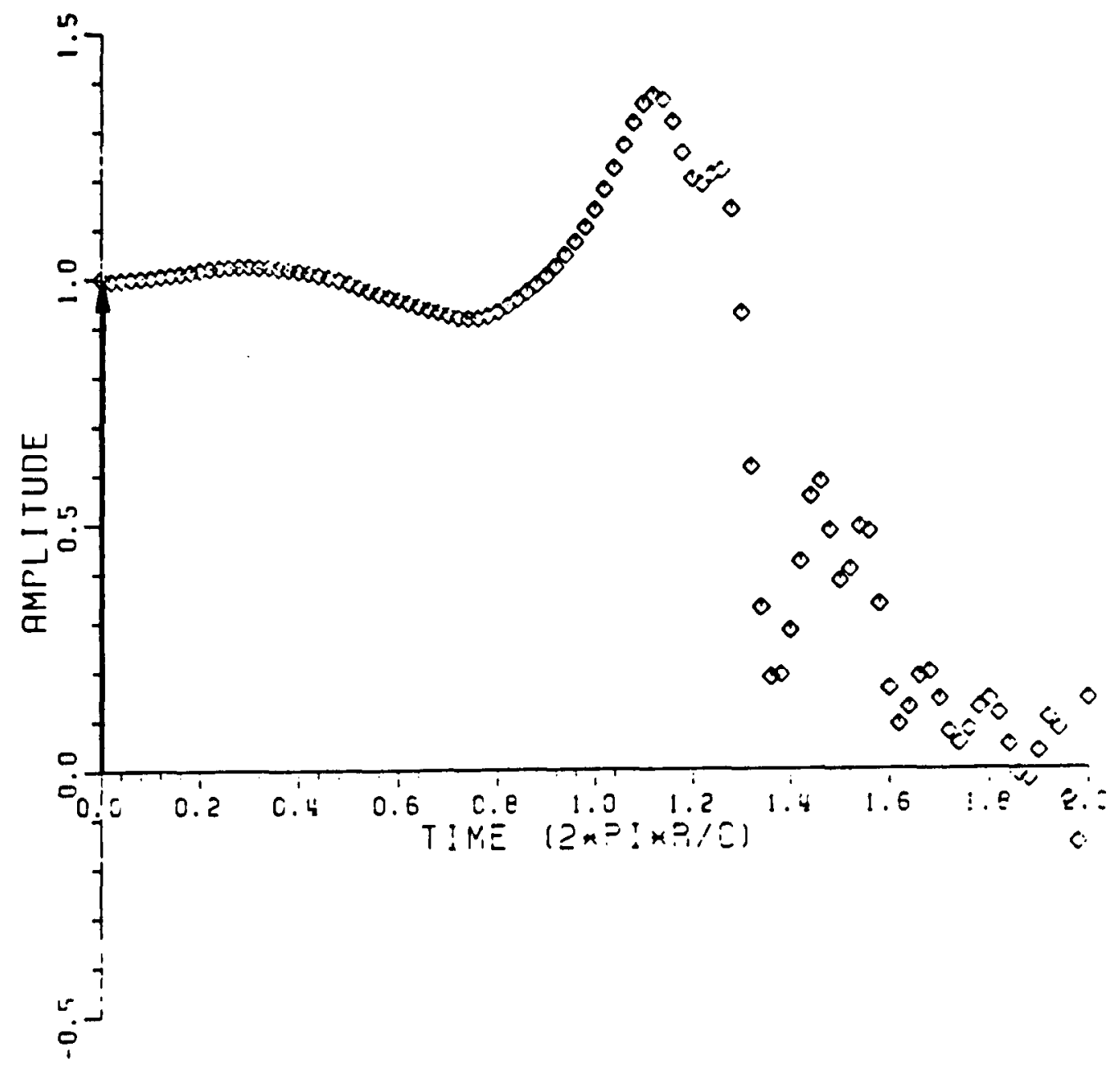

Figure 4.27. K-pulse obtained using the Fourier synthesized impulse response of the conducting circular disk at $\theta=45^{\circ}$, $\hat{\theta}$-polarization with $k r=0.16(0.16) 16$. The $K$-pulse duration is $4 \pi r / c$. 


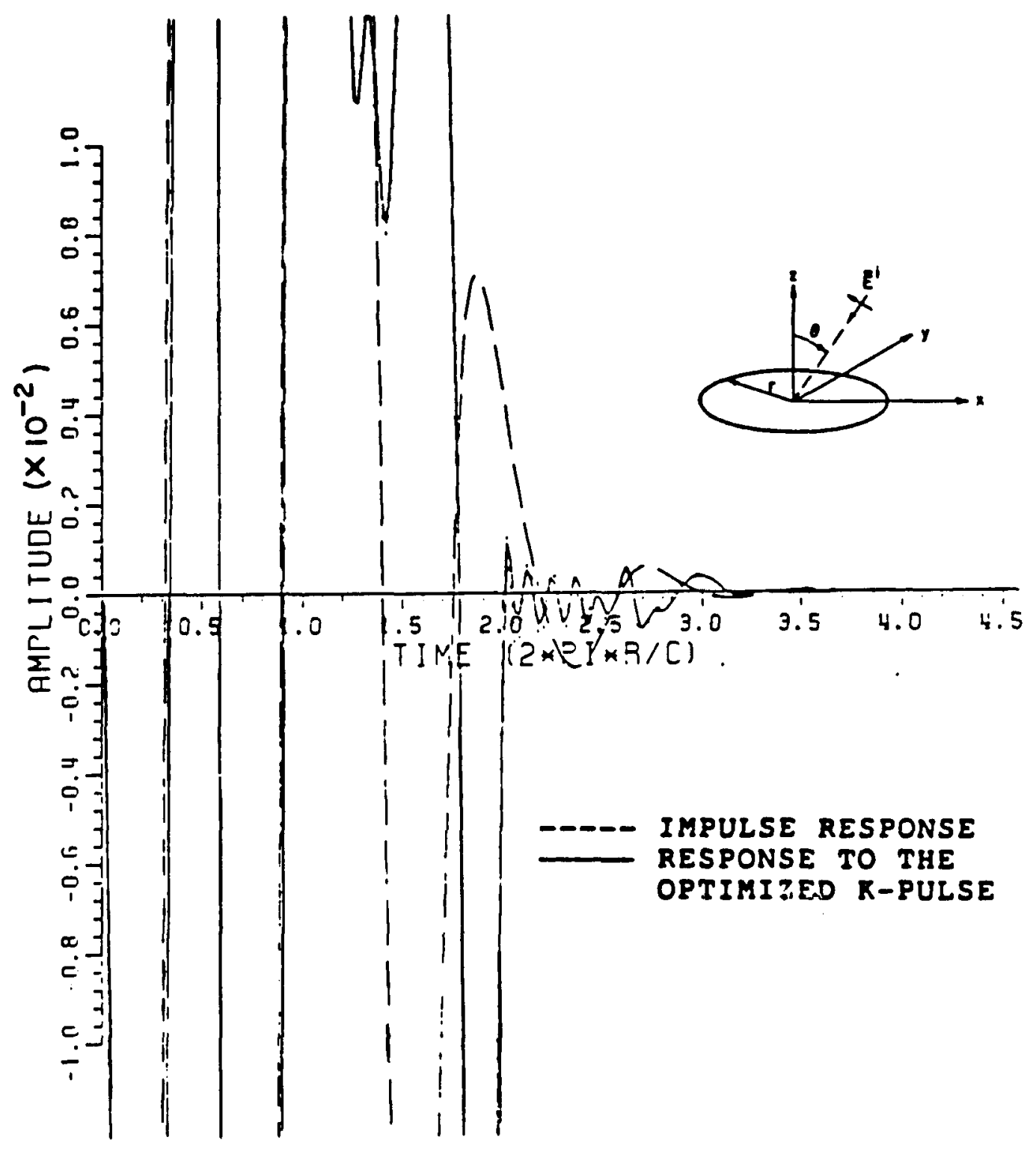

Figure 4.28. Impulse response and response to the K-pulse shown in Figure 4.27 for the conducting circular disk at $\theta=45^{\circ}$, $\hat{\theta}$-polarization with $\mathrm{kr}=0.16(0.16) 16$. The amplitude scale is expanded by 100 . 


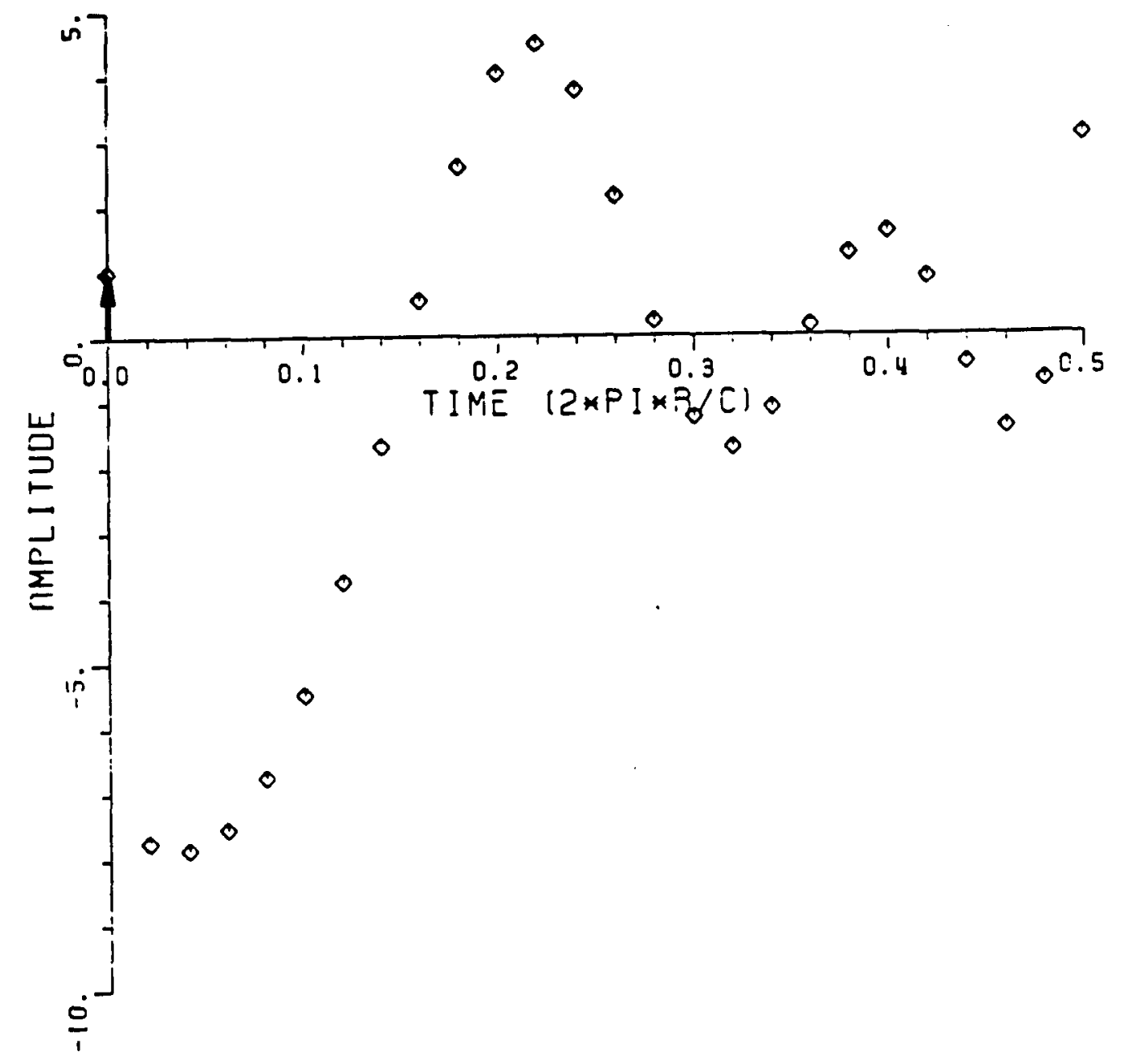

Figure 4.29. "K-pulse" obtained using the Fourier synthesized impulse response of the conducting circular disk at $\theta=45^{\circ}$, $\theta$-polarization with $k r=0.16(0.16) 16$. The "K-pulse" duration is $\pi r / c$. 


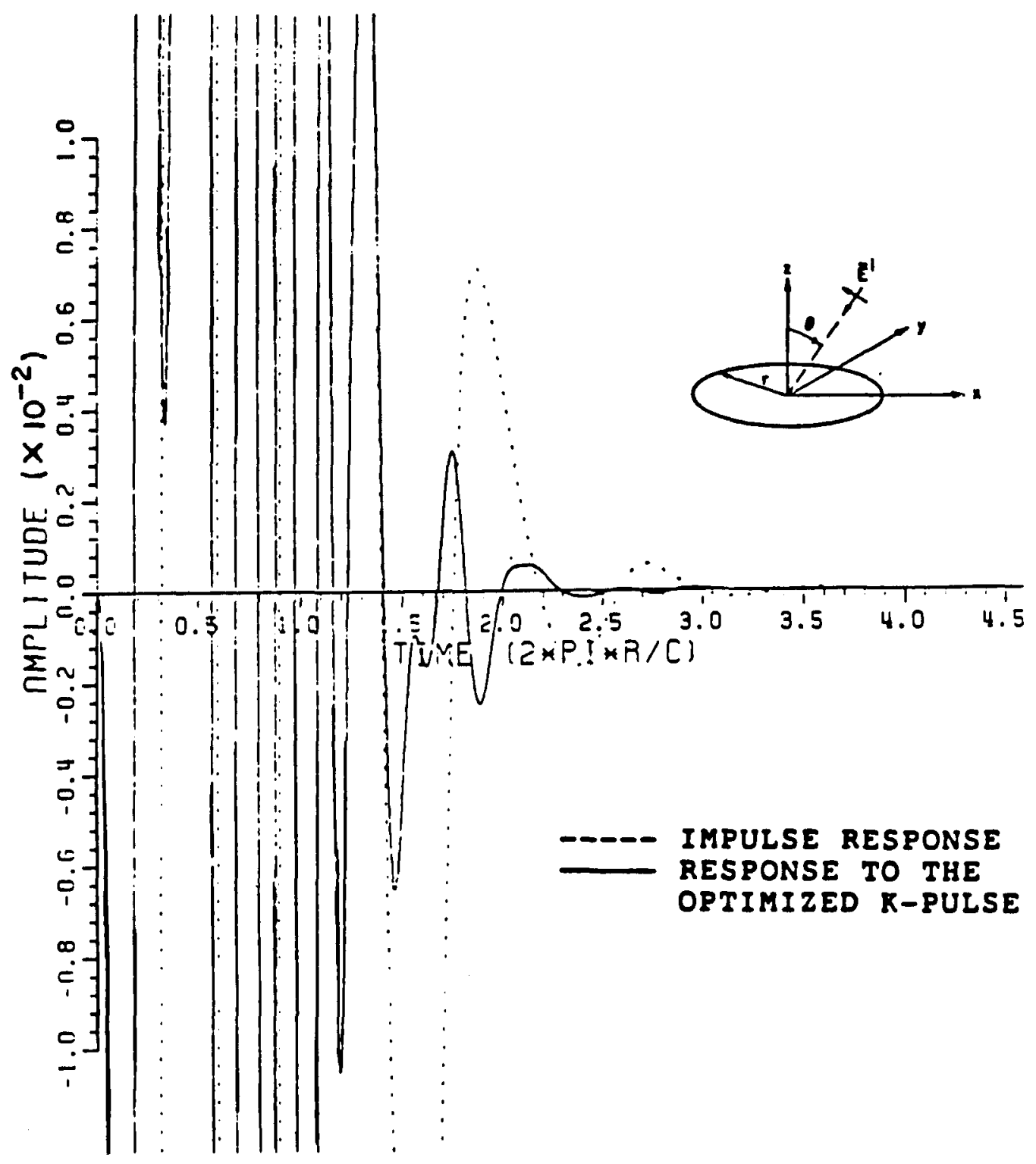

Figure 4.30. Impulse response and response to the "K-pulse" shown in Figure 4.29 for the conducting circular disk at $\theta=45^{\circ}$, $\hat{\theta}$-polarization with $\mathrm{kr}=0.16(0.16) 16$. The amplitude scale is expanded by 100 . 
CHAPTER $V$

APPLICATION TO NOISY AND MEASIIREN DATA

In this chapter, the theory discussed in Chapter II is applied to simulated noisy data and measured data. The simulated noisy data are generated by the addition of white Gaussian notse of zero mean to the theoretical data. The Gaussian notse is generated with the subroutine GAUSS in IBM System/360 Scientific Subroutine Package Version III. The theoretical data for the circular loop are used for this study. The measured data are from two finite circular waveguides, whose geometries are the same and shown in Figure 5.1. (For the convenience of the reader, all tables and figures of Chapter $V$ are grouped together at the end of the chapter.) One of the circular waveguides has both ends opened. The other waveguide has the end at $\phi=180^{\circ}$ shorted. The measurement of the geometries was done on the Ohio State University Compact Range Reflectivity Facility [26].

The theoretical circular loop data are the same as in the previous chapters. The ratio of the wire radius (a) to the loop radius ( $r$ ) is $\pi \times 10^{-3}$. The bandwidth of the data is $k r=0.02 \pi(0.02 \pi) 4 \pi$. The aspect angle and polarization of the impulse response used are $\theta=45^{\circ}$ and $\hat{\theta}$-polarization, respectively. The noise is added independently into the real and imaginary parts of the frequency data before discrete Fourter transforming the data into the time domain. The maximum amplitude of the real and imaginary parts in the frequency data are about $0.36 \mathrm{~V}$ and 
$0.62 \mathrm{~V}$, respectively. For the first test the added Gaussian noise has a zero mean and a standard deviation of $0.01 \mathrm{~V}$.

The impulse response obtained from the polluted data is shown in Figure 5.2. The periodicity at late time of the impulse response is about $2 \pi r / C$, which is the proper $K$-pulse duration (T). The optimization process is started with 50 as the number of samples (N) to respresent the continuous portion of the K-pulse. The energy is minimized over the period from $6 \pi r / c$ to $12 \pi r / c$. After ten steps of Powell-Fletcher type of minimization, the value of $N$ is increased to 100 , and the $K$-pulse response duration is reduced to $5 \pi r / c$. The cutoff time for the integration is still $12 \pi r / c$. After another ten steps of the PowellFletcher type of optimization, the resulting $K$-pulse is shown in Figure 5.3. Again the first diamond is an impulse, which is highlighted by an arrow, the other one hundred diamonds are samples of a continuous function. The impulse response and the response to the $K$-pulse in Figure 5.3 for the loop at $\theta=45^{\circ}$ and $\hat{\theta}$-polarization are plotted in Figure 5.4. The energy content remaining in the $K$-pulse response later than $t=4 \pi r / c$ appears to be contributed by the noise component, as the amplitude is in the noise level.

To see how well the the K-pulse obtained (Figure 5.3) stops the resonance at late time, this $K$-pulse is convolved with the noiseless impulse responses. By eliminating the noise factor, the K-pulse response can be be compared with the results in Chapter III. Figure 5.5 contains both the impulse response and the response to the K-pulse shown in Figure 5.3 for the loop at $\theta=45^{\circ}$ and $\hat{\theta}$-polarization without noise. 
The energy content after $t=4 \pi r / c$ is very small. Figure 5.6 is the impulse response and the response to the K-pulse shown in Figure 5.3 for the loop at $\theta=0^{\circ}$ and $\hat{\phi}$-polarization. Again the $K$-pulse response has very little energy after $2.4 \pi r / c$. The impulse response and the response to the K-pulse shown in Figure 5.3 for the loop at $\theta=45^{\circ}, \hat{\phi}$-polarized are plotted in Figure 5.7. There is only a small residue of energy after $4 \pi r / c$. The response to the K-pulse shown in Figure 5.3 and the impulse response for the loop at $\theta=90^{\circ}, \hat{\phi}$-polarization are shown in Figure 5.8. The amplitude of the $K$-pulse response signal after $5 \pi r / C$ is greatly reduced. If Figures 5.5 through 5.8 are compared with Figures 3.18 through 3.21 , respectively, the K-pulse obtained here is performing very competitively as the K-pulse in Figure 3.17 does. That is why it is not surprising to see the dominant zeros of this K-pulse (Figure 5.3) compare very well with the true poles of the circular loop as shown in Table 5.1. The notse level specified in this example is fairly close to the notse level encountered at the 0.S.U. compact range facility. This can be seen by comparing the $K$-pulse response at late time in Figure 5.4 with Figure 5.24 where the noise amplitude is fluctuating between $\pm 0.03 \mathrm{~V}$.

In the second example, the noise level of the signal is increased five times. This is to evaluate the K-pulse estimation technique when the signal is corrupted by a large amount of noise. The frequency response for the same circular loop at $\theta=45^{\circ}, \hat{\theta}$-polarization is again corrupted with white Gaussian noise of zero mean with standard deviation of $0.05 \mathrm{~V}$. The impulse response is shown in Figure 5.9. The periodicity 
of the signal at late time is stfll around $2 \pi r / c$. Thus, the K-pulse duration $(T)$ is still chosen as $2 \pi r / C$. The $K$-pulse response duration is chosen as $5 \pi r / c$. The cutoff time for integration is $12 \pi r / c$. The number of samples (N) used to represent the continuous portion of the $K$-pulse is 100. After six steps of Powell-Fletcher minimization, the K-pulse as shown in Figure 5.10 is obtained. The impulse response and response to the K-pulse shown in Figure 5.10 for the loop at $\theta=45^{\circ}$, and $\hat{\theta}$-polarization are shown in Figure 5.11. The K-pulse response after $4 \pi r / c$ is indeed very noisy. To check the approximate $K$-pulse, it is again convolved with notseless data. The K-pulse is first convolved with the noiseless impulse response at $\theta=45^{\circ}, \hat{\theta}$-polarization. The result is contrasted with the impulse response in figure 5.12. There are still some residues after $4 \pi r / c$. This is to be expected, as the noise level of the impulse response employed to obtain the $K$-pulse has been increased. The performance of the technique is expected to degrade. Similar late time behaviour is exhibited at the other aspect angles and polarizations. Figure 5.13 shows the impulse response and the response to the K-pulse shown in Figure 5.10 for the loop at $\theta=0^{\circ}$, $\hat{\phi}$-polarization. Figures 5.14 and 5.15 show both the impulse response and the response to the K-pulse shown in Figure 5.10 for the loop at $\hat{\phi}$-polarization with $\theta=45^{\circ}$ and $90^{\circ}$, respectively. Even though Figures 5.12 through 5.14 have some residue at late time, the majority of the energy contained in the late time of the impulse response has been reduced. The first few dominant poles are probably available from the zeros of the K-pulse in Figure 5.10. This is confirmed in Table 5.1. 
In fact, the first two zeros are very close to the true poles. Furthermore, all of the imaginary parts of the zeros are very close to the corresponding true poles.

In this next example, some backscattered measurement data are examined. The target is a finite circular waveguide with open ends. The geometry of the waveguide is shown in Figure 5.1. The measurement is taken over the bandwidth of $k r=1.32(0.00665) 12$, where $r$ is the radius of the waveguide. At $\phi=0^{\circ}, \theta=0^{\circ}$, a moment method solution [27] is used to supplement the measured frequency data making the effective bandwidth of the data as $k r=12$. The impulse response for the open-ended finite circular waveguide at $\phi=0^{\circ}, \theta=0^{\circ}$ is shown in Figure 5.16. Figure 5.16 shows that there are two major scattering centres in the impulse response - one at the front of the waveguide at $t=0$; the other at the back of the waveguide at $t=6.8 \pi r / c$. The earlier portion $(0<t<6 \pi r / c)$ of Figure 5.16 can be viewed as the return from a semi-infinite circular waveguide, since the effect from the back of the waveguide cannot be within this early period. The amplitude at later time $(t>6 \pi r / c)$ of the impulse response, of course, is the character of the finite open-ended finite circular waveguide. Thus, the impulse response can be divided into sections to isolate each of these mechanisms. In other words, this example can be further divided into two sub-sections - one for the semi-infinite circular waveguide $(0<t<6 \pi r / c)$, another for the finite circular waveguide $(t>6 \pi r / c)$. The portion of the impulse response related to the semi-infinite circular waveguide is analyzed first. Then the analysis of the impulse response for the late time of the finite circular waveguide is discussed. 
The early portion of the impulse response of the finite circular waveguide is plotted in figure 5.17. The approximate periodicity is $\pi r / c$; therefore, the $K$-pulse duration is chosen as $\pi r / c$. The energy is minimized between $3 \pi r / c$ to $5 \pi r / c$. After five steps of Powell-Fletcher minimization, the K-pulse is as shown in figure 5.18. The response to the $K$-pulse shown in Figure 5.18 and impulse response for the semi-infinite circular waveguide at $\phi=0^{\circ}, \theta=0^{\circ}$ are shown in Figure 5.19. The periodicity between $3 \pi r / c$ to $5 \pi r / c$ is gone. The residual amplitude at late time seems to be contributed by noise. The zeros of the K-pulse are listed in Table 5.2. Two of them are simflar to what Moffatt et al. [28] presented as complex natural resonances of a semi-infinite circular waveguide extracted using rational function approximants; namely, $-2.076+11.266 j$ and $-5.0146+38.590 j$.

Measurements were also performed on a simflar circular waveguide with one end closed. The same bandwidth of $k r=1.32(0.00665) 12$ is used, where $r$ is the radius of the waveguide. Similarly, a moment method solution [27] is supplemented in the lower frequency range to give the impulse response at $\phi=0^{\circ}, \theta=0^{\circ}$ a bandwidth of $k r=12$. The closed end is at $\phi=180^{\circ}$. Since the early time $(0<t<6 \pi r / c)$ of this impulse response does not contain the effect of the closed end, this section of the impulse response (Figure 5.20) can also be considered as the effect of the semi-infinite circular waveguide. However, the impulse response of the circular waveguide with one closed end seems to have a slight error. The amplitude is decaying until about $3.4 \pi r / c$, then the signal seems to be growing. There are some energy reflection 
from the back before the wave reaches the shorted end of the circular waveguide. This may be due to the alignment of the circular waveguide at the time of the experiment. Figure 5.21 is the impulse response and the response due to the $K$-pulse of Figure 5.18 for the semi-infinite circular waveguide at $\phi=0^{\circ}$ using the data of the finite circular waveguide with one closed end. The K-pulse of Figure 5.18 is doing a reasonable job of terminating the resonances except between $4 \pi r / c$ to $6 \pi r / c$. Taking the error described earlier into consideration, the slight increase in energy between $4 \pi r / c$ to $6 \pi r / c$ can be regarded as measurement error rather than a problem with the K-pulse. However, this K-pulse does not completely terminate the late time resonance of the open-ended circular waveguide at $\phi=0^{\circ}, \theta=0^{\circ}$ (FIgure 5.22). In other words, the type of resonance exhibited by the semi-infinite circular waveguide is different from the finite circular waveguide at late time.

To find the $K$-pulse to $k i l l$ the resonance at the late time of the open-ended circular waveguide, the impulse response of the waveguide at $\phi=0^{\circ}, \theta=0^{\circ}$ (Figure 5.16) is restudied. The periodicity of the impulse response at late time is still $\pi r / c$, so the $K$-pulse duration is also chosen as such. The energy between $10 \pi r / c$ and $16 \pi r / c$ is minimized with steps of Powell-Fletcher Scheme. After three steps of optimization, the K-pulse shown in Figure 5.23 is obtained. The impulse response and the response to the $K$-pulse shown in Figure 5.23 for the finite open-ended circular cylinder are compared in Figure 5.24. The amplitude of the $K$-pulse response after $8.8 \pi / c$ is in the noise level. The noise level judging from the very late time amplitude is fluctuating between $\pm 0.02 \mathrm{~V}$. 
There is a little hump at about $14 \pi / c$ which is very close to $13.6 \pi r / c$, the second time that the signal travels to and from the back of the waveguide. There also seems to be a slight hump around $20.4 \pi r / c$, the third time that the impulsive signal goes to the back of the waveguide and returns. The K-pulse (Figure 5.23) does not cancel this mechanism because the $k$-pulse duration is chosen as $\pi r / c$. To kill this mechanism requires the duration of the $K$-pulse be increased to $6.4 \pi r / c$. Since the magnitude of this mechanism is not large, its contribution is neglected. The poles associated with the late time are listed in Table 5.2.

The convolution of the K-pulse (Figure 5.23) with the $\phi=0^{\circ}, \theta=0^{\circ}$ impulse response of the circular waveguide with one closed end at $\phi=180^{\circ}$, is shown in figure 5.25 . It is not surprising to see that the ampltude of the $K$-pulse response at late time $(t>11 \pi r / c)$ has been greatly reduced. For this configuration, the signal received is very simflar to the bistatic scattering at $\phi=180^{\circ}, \theta=0^{\circ}$ for an open-ended circular waveguide with twice the length. The wave at the origin is actually the same in the two cases because of image theory. Therefore, the contributors to the resonance come from the different waveguide modes excited. According to C. C. Huang's [29] calculation, there can be as many as 40 different modes excited inside the waveguide. Consequently, there is a lot of energy between $t=6.8 \pi r / c$ and $t=11 \pi r / c$ in the $K$-pulse response. It is also interesting to note that there is a little hump around $t=15.6 \pi r / c$, which is the second round trip time for the signal to travel to the closed end and back. 
A few words about the convergence of the K-pulses obtained in this chapter is discussed next. Using the impulse response at $\phi=0^{\circ}, \theta=0^{\circ}$ for the circular waveguide with one closed end, the K-pulse obtained from the late time $(t>11 \pi r / c)$ has a shape very similar to the K-pulse presented in Figure 5.23. One can only expect them to be very close, as notse is involved. It is very unlikely that they would be the same. Since the early time $(0<t<3 \pi r / c)$ of this impulse response has some slight errors, the K-pulse obtained from the early time is quite different from Figure 5.18. Other aspect measurements are also avaflable; but the bandlimited data does not seem to exhibit a lot of resonance.

In this chapter, the K-pulse estimation technique performs well with the quasi-noisy data. In fact, for a noise level that is similar to the 0.S.U. compact range facility, the technique works extremely well. The dominant poles extracted are very close to the exact values. Even for very notsy data, the first two poles are still available from the approximate K-pulse. The imaginary parts for all the poles extracted are very close to the corresponding exact imaginary parts of the poles. Also, this chapter has demonstrated that by isolating the resonant mechanism in the time domain, the poles and the K-pulses associated with each scattering mechanism can be extracted. Using the finite circular waveguide data, the $K$-pulse associated with the semi-infinite circular waveguide has been derived by isolating that portion corresponding to the semi-infinite circular waveguide. 
Table 5.1

A Comparison of the First Six Exact Poles and Estimated Zeros in the s-domain (Upper Left Half Plane) for the Thin Circular Loop
Exact
$\sigma=0.01 \mathrm{~V}$
$\underline{\sigma}=0.05 \mathrm{~V}$
1) $-0.423+6.509 j$
$-0.459+6.500 j$
$-0.433+6.340 j$
2) $-0.606+12.871 j$
$-0.639+12.883 j$
$-0.590+12.710 j$
3) $-0.745+19.211 j$
$-0.715+19.320 j$
$-0.332+19.170 j$
4) $-0.862+25.541 j$
$-0.972+25.605 j$
$-1.405+26.104 j$
5) $-0.965+31.864 j$
$-1.348+32.516 j$
$-1.836+33.032 j$
6) $-1.059+38.183 j$
$-1.727+38.933 j$
$-2.738+39.308 j$ 
Table 5.2

The First Five Estimated Poles in the s-domain (Upper Left Half Plane) for the Finite Circular Waveguide

$\begin{array}{lcc} & \text { Early Time } & \text { Late Time } \\ \text { 1) } & -1.319+11.457 j & -0.384+13.728 j \\ \text { 2) } & -6.815+25.319 j & -6.532+28.861 j \\ \text { 3) } & -4.074+36.246 j & -7.084+40.139 j \\ \text { 4) } & -5.668+52.065 j & -7.564+54.135 j \\ \text { 5) } & -6.790+65.024 j & -8.409+67.192 j\end{array}$




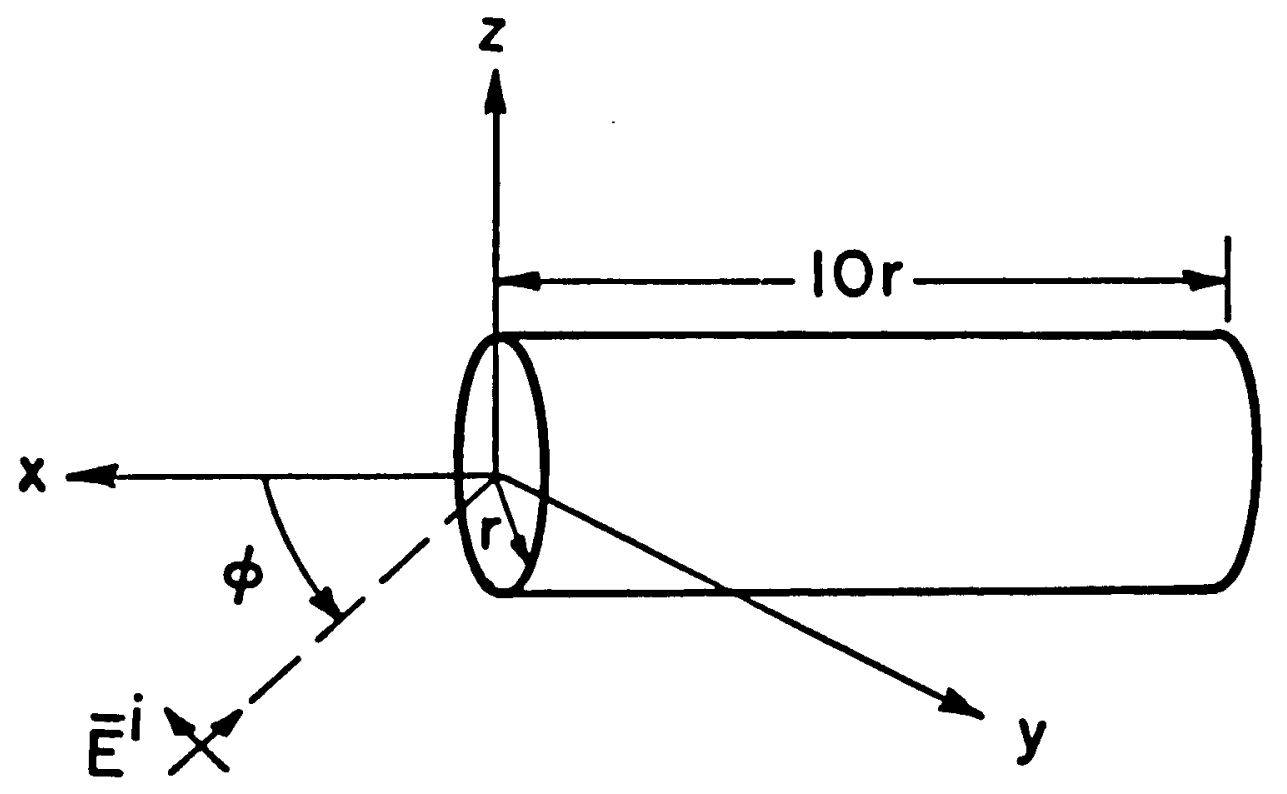

Figure 5.1. An fllustration of the geometry of a finite circular waveguide. 


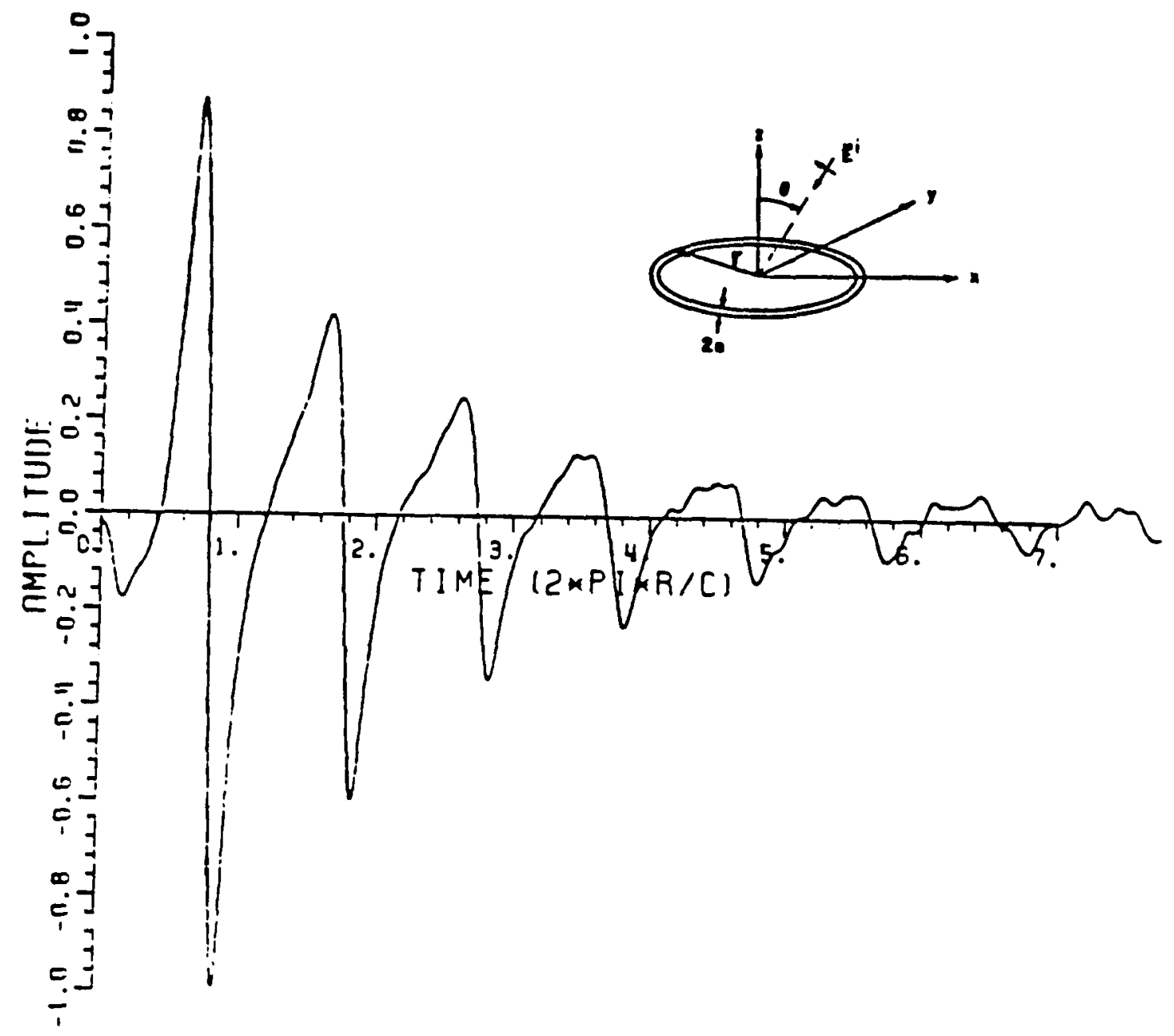

Figure 5.2. Fourter synthesized impulse response of the circular loop at $\theta=45^{\circ}, \hat{\theta}$-polarization with Gaussian notse $N(0,0.01 \mathrm{~V})$. $(k r=0.02 \pi(0.02 \pi) 4 \pi)$. 


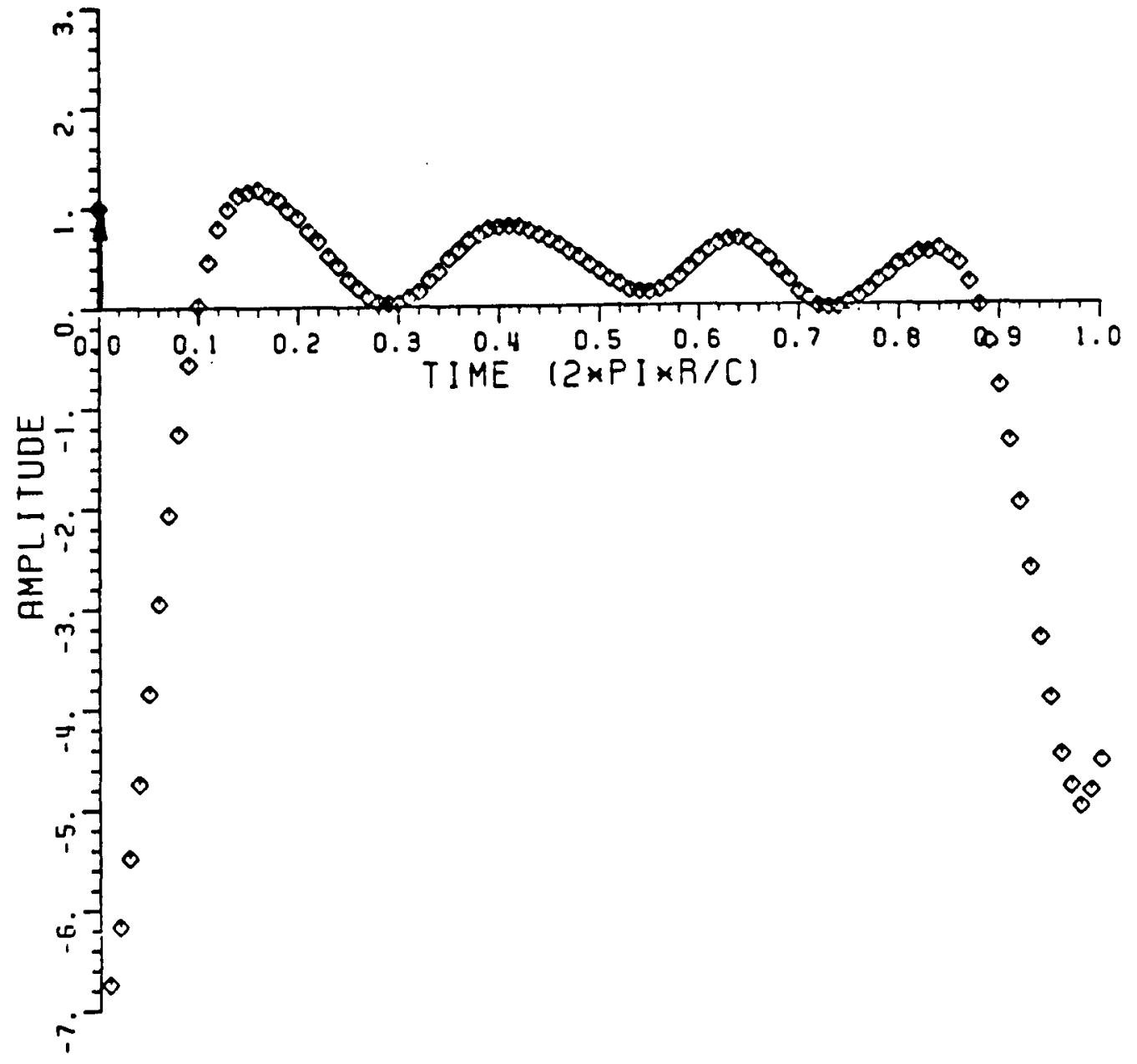

Figure 5.3. K-pulse obtained using the impulse response shown in Figure 5.2. 


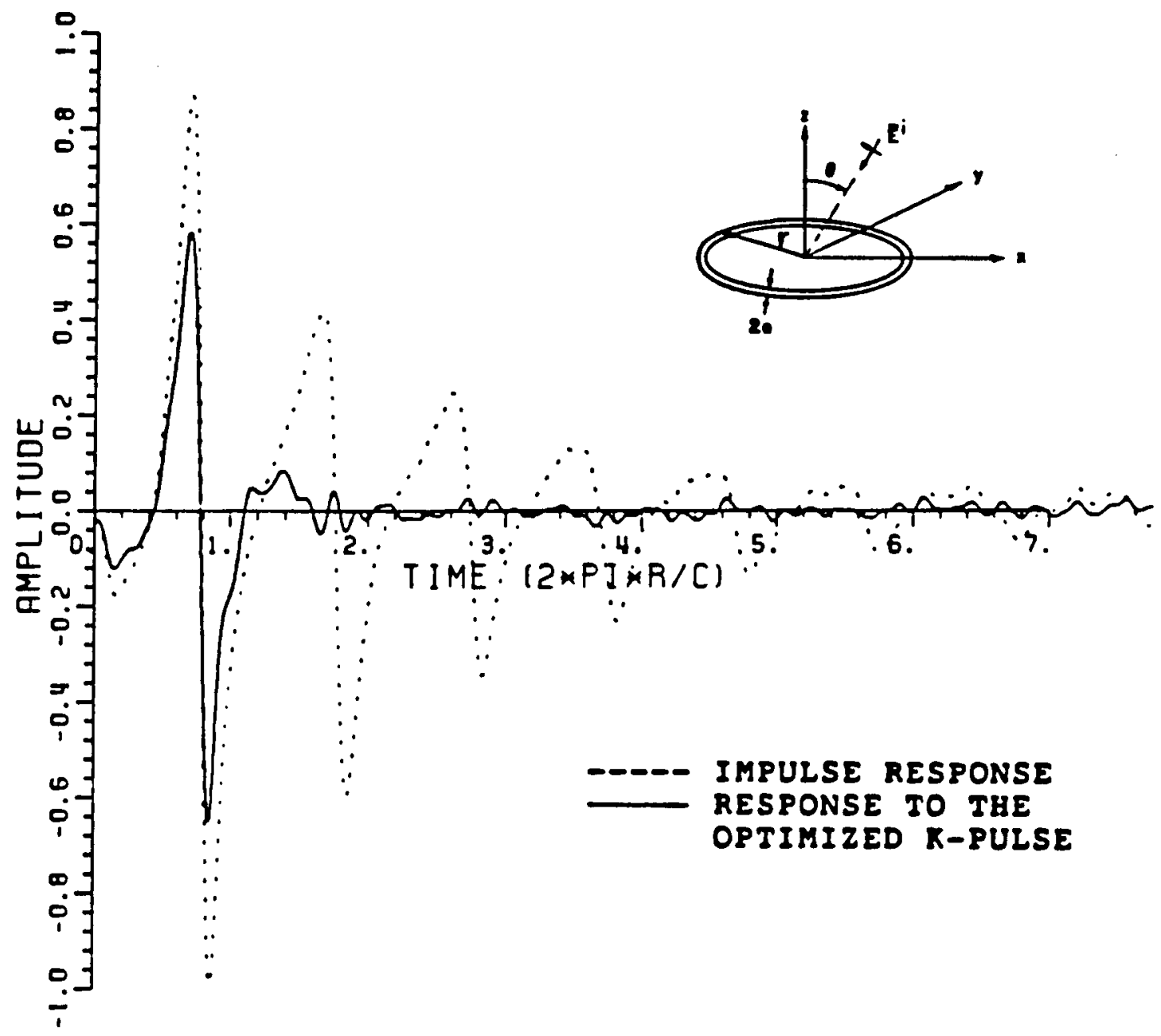

Figure 5.4. Impulse response and response to the K-pulse shown in Figure 5.3 for the circular loop at $\theta=45^{\circ}, \hat{\theta}$-polarization with Gaussian noise $N(0,0.01 V)$. ( $k r=0.02 \pi(0.02 \pi) 4 \pi)$. 


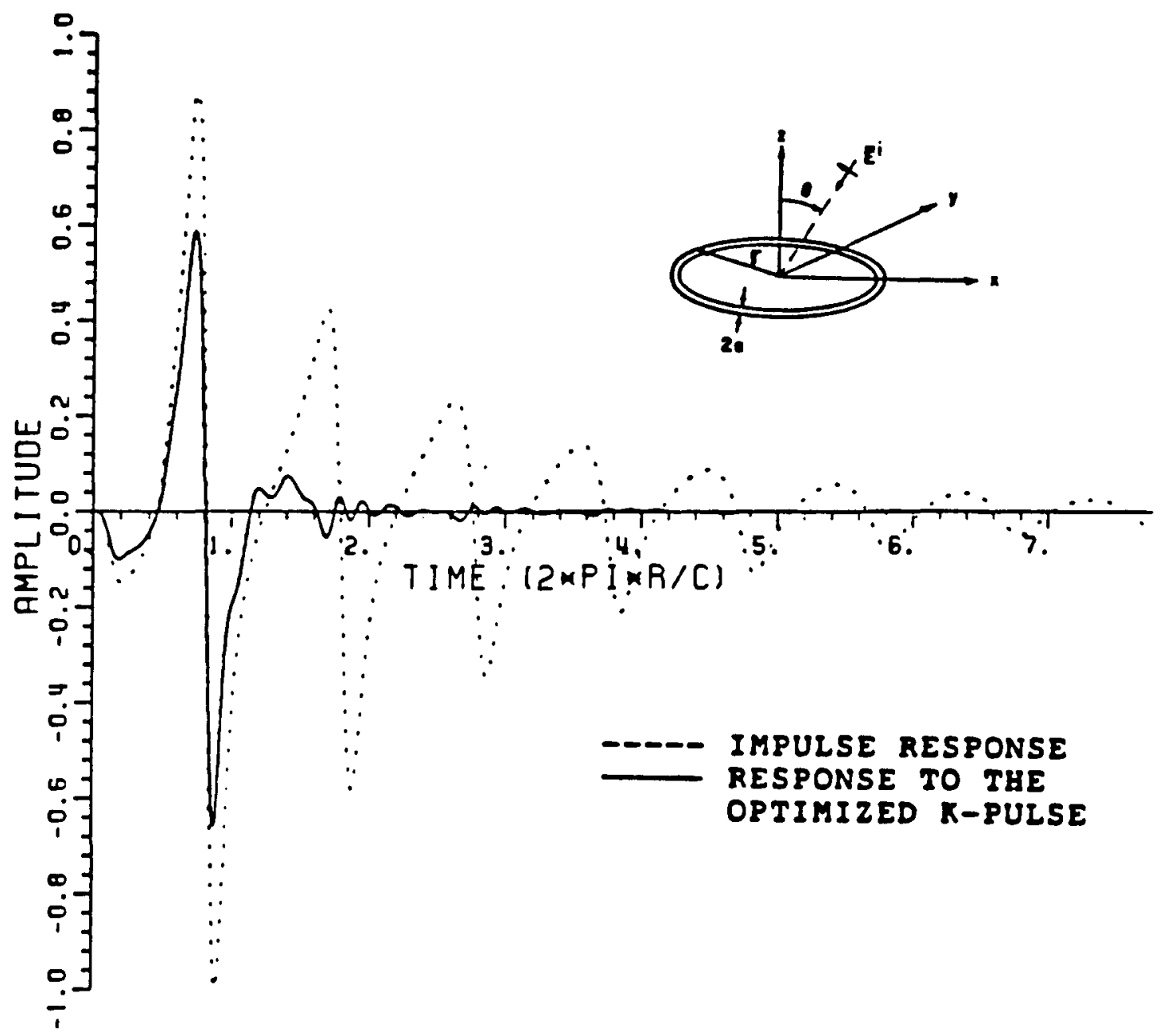

Figure 5.5. Impulse response and response to the $K$-pulse shown in Figure 5.3 for the circular loop at $\theta=45^{\circ}, \hat{\theta}$-polarization with no noise. $(k r=0.02 \pi(0.02 \pi) 4 \pi)$. 


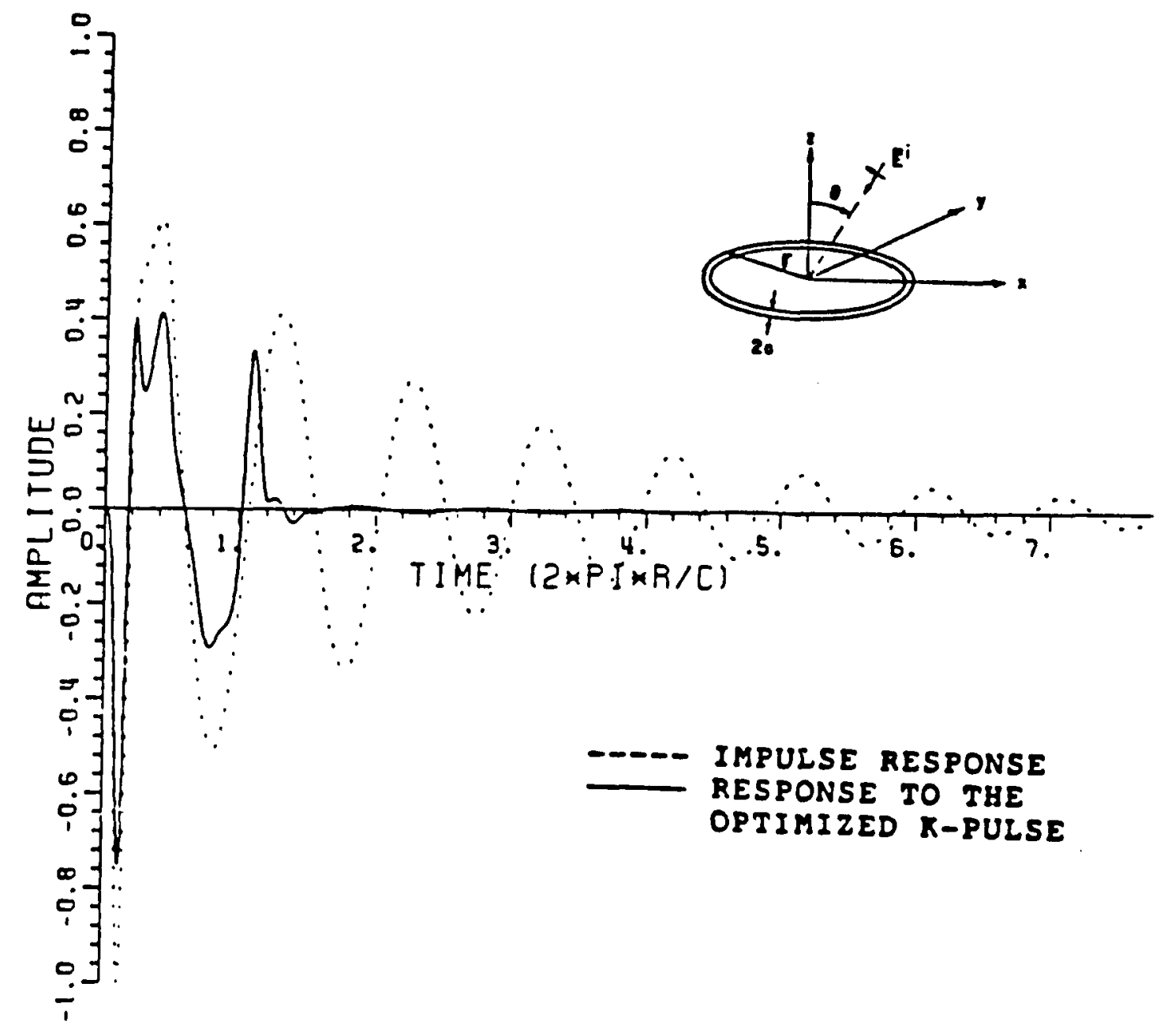

Figure 5.6. Impulse response and response to the $K$-pulse shown in Figure 5.3 for the circular loop at $\theta=0^{\circ}, \hat{\phi}$-polarization with no noise. $(k r=0.02 \pi(0.02 \pi) 4 \pi)$. 


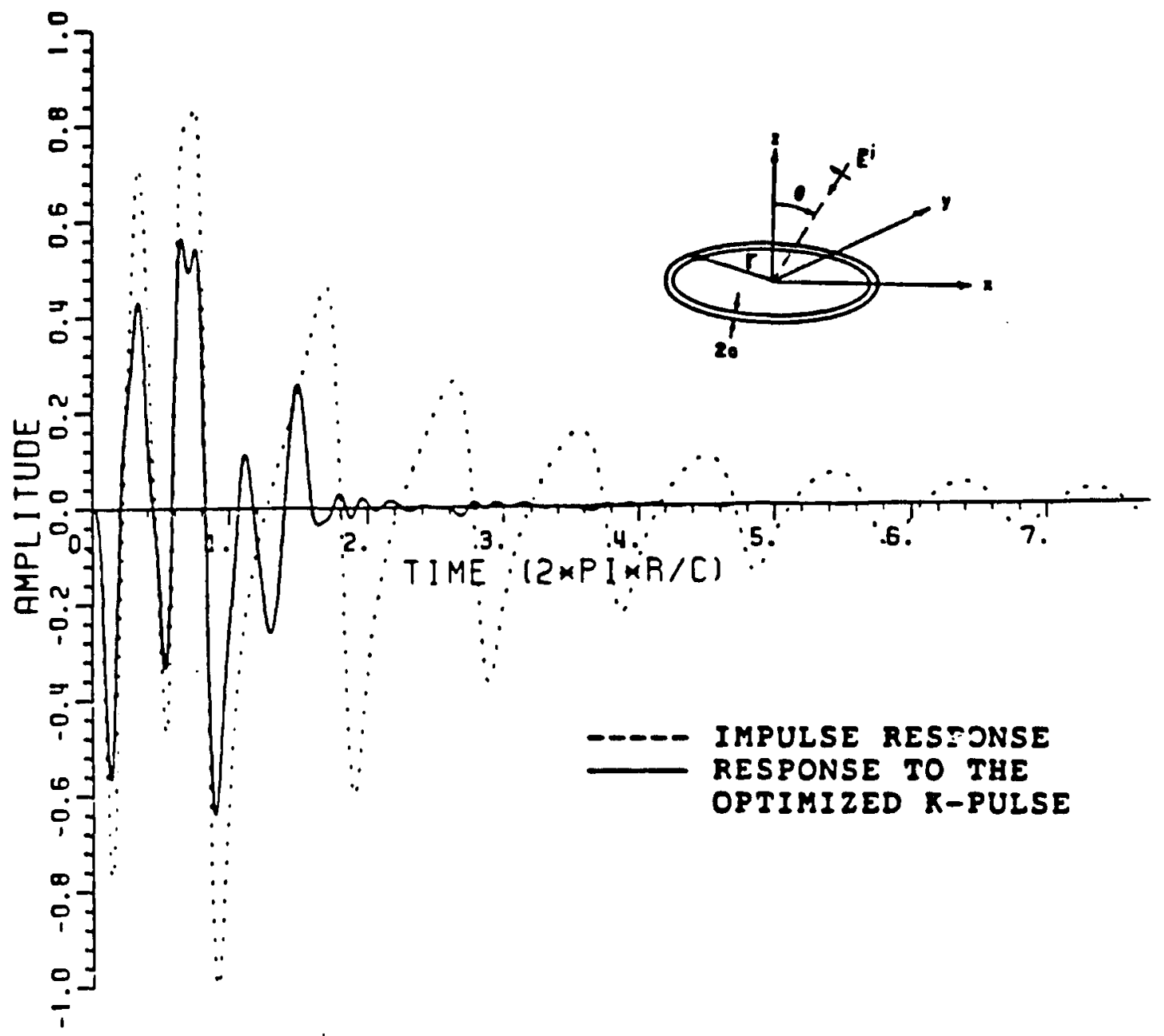

Figure 5.7. Impulse response and response to the $K$-pulse shown in Figure 5.3 for the circular loop at $\theta=45^{\circ}, \hat{\phi}$-polarization with no noise. $(k r=0.02 \pi(0.02 \pi) 4 \pi)$. 


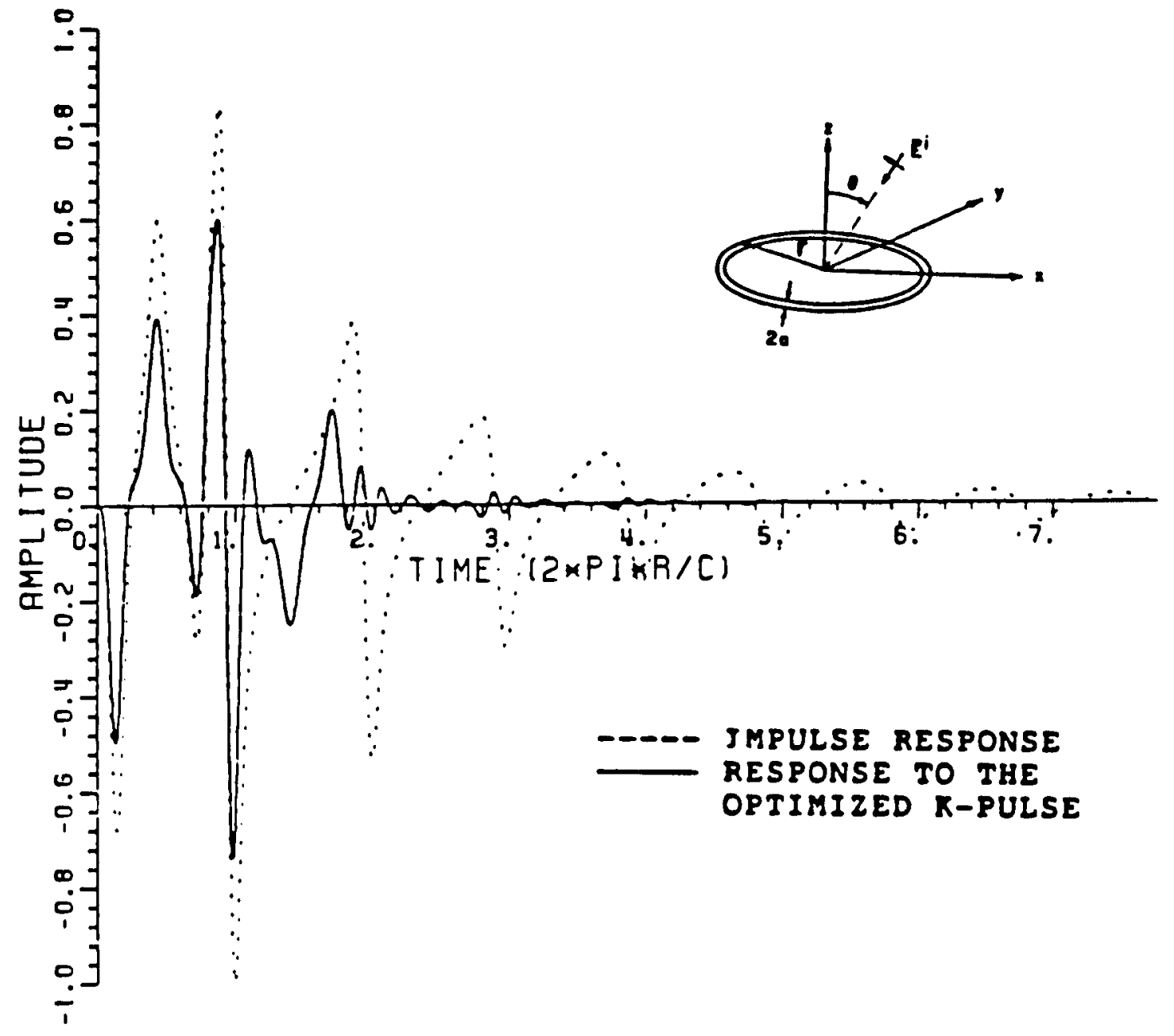

Figure 5.8. Impulse response and response to the $K$-pulse shown in Figure 5.3 for the circular loop at $\theta=90^{\circ}, \hat{\phi}$-polarization with no noise. $(k r=0.02 \pi(0.02 \pi) 4 \pi)$. 


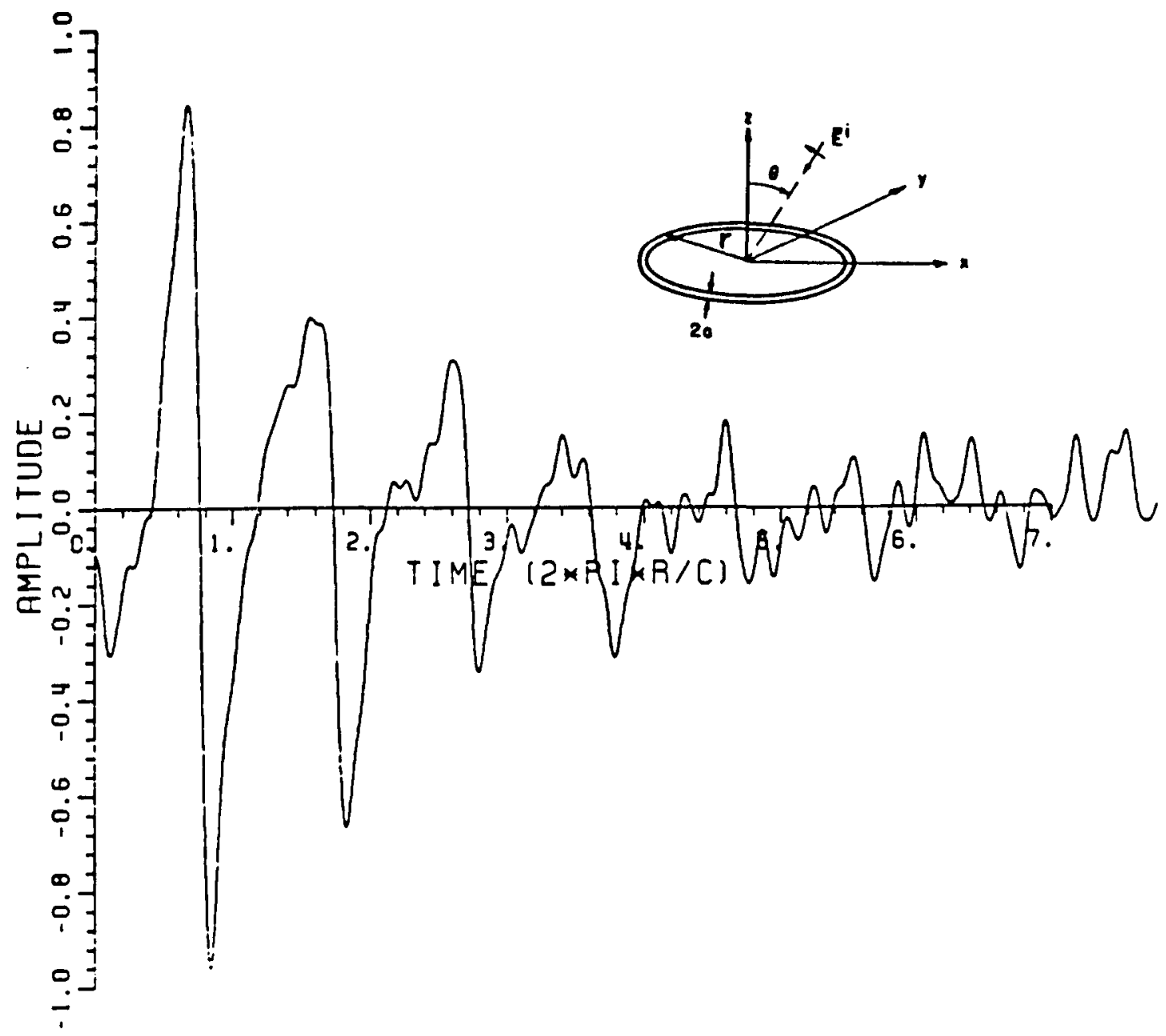

Figure 5.9. Fourier synthesized impulse response of the circular loop at $\theta=45^{\circ}, \hat{\theta}$-polarization with gaussian notse $N(0,0.05 \mathrm{~V})$. $(k r=0.02 \pi(0.02 \pi) 4 \pi)$. 


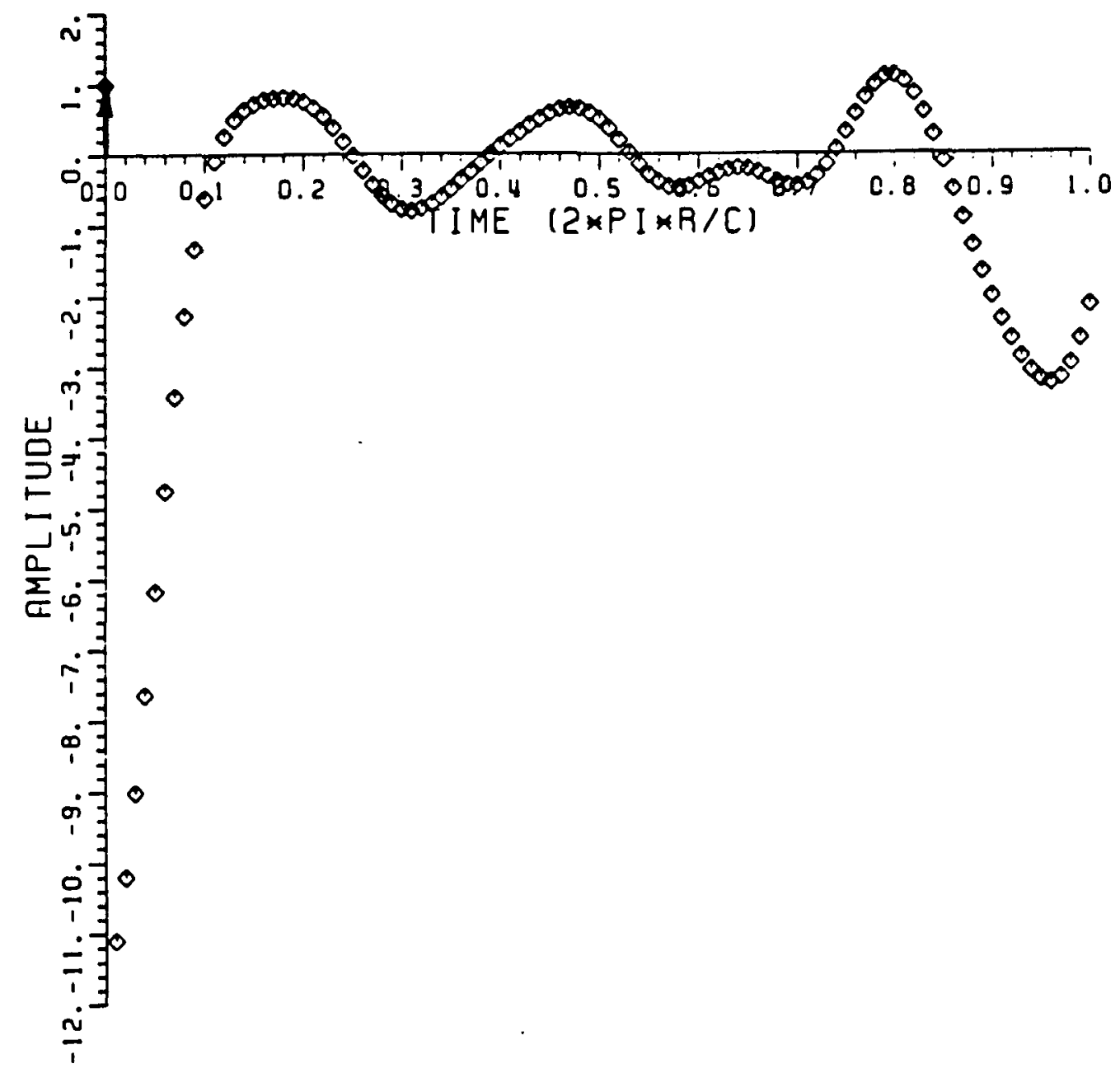

Figure 5.10. K-pulse obtained using the impulse response shown in Figure 5.9. 


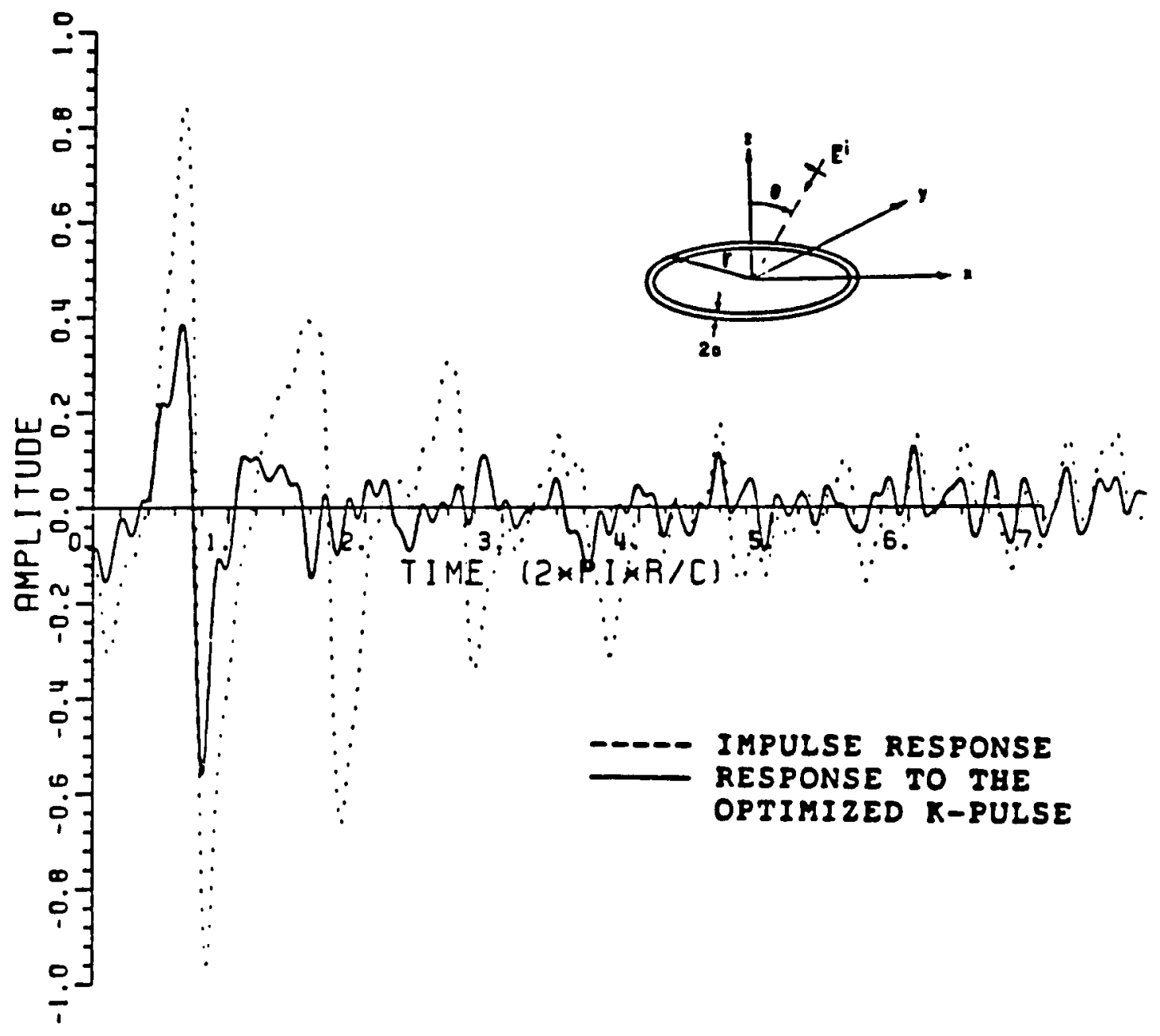

Figure 5.11. Impulse response and response to the $K$-pulse shown in Figure 5.10 for the circular loop at $\theta=45^{\circ}, \hat{\theta}$-polarization with Gaussian noise $N(0,0.05 V) . \quad(k r=0.02 \pi(0.02 \pi) 4 \pi)$. 


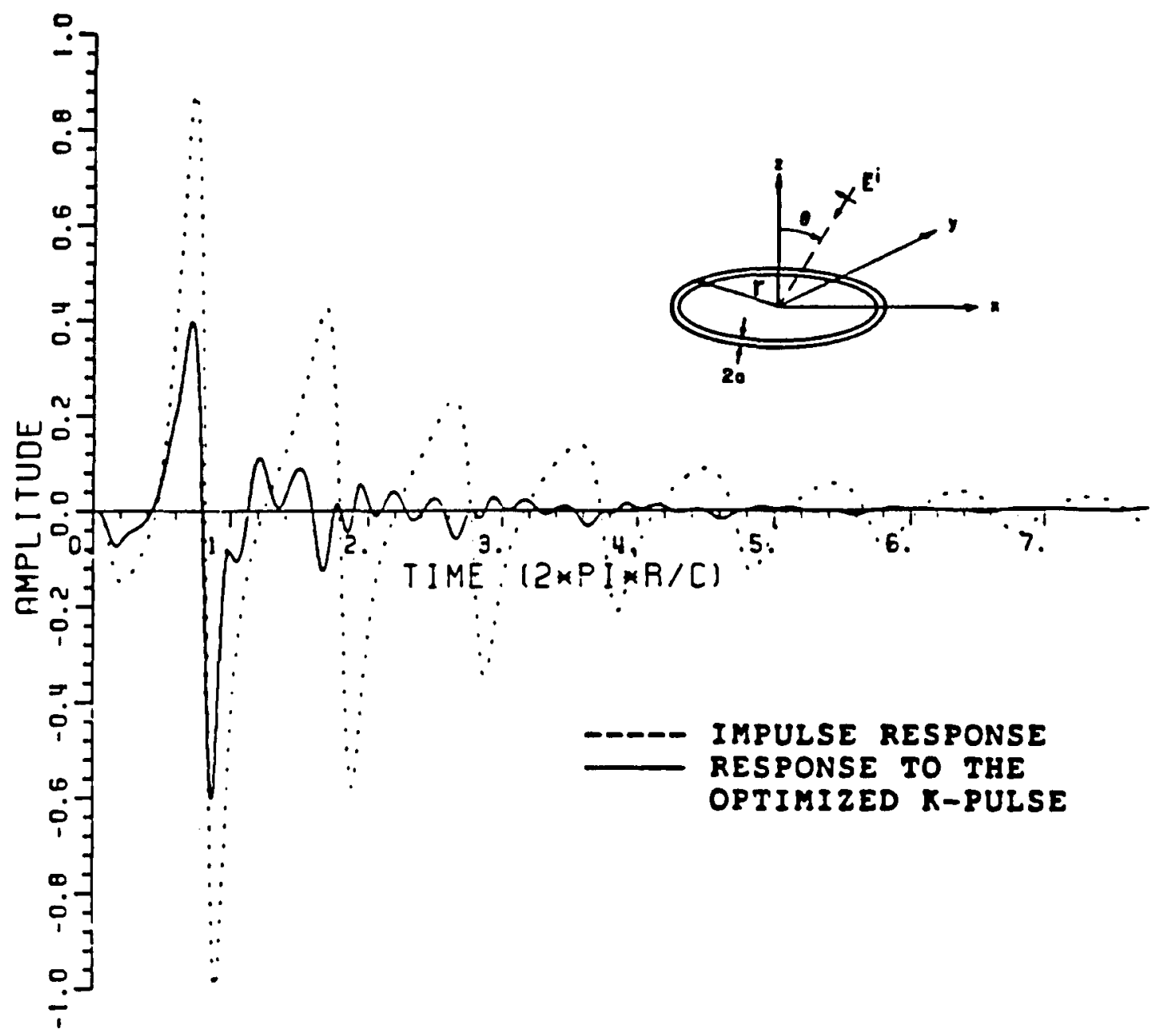

Figure 5.12. Impulse response and response to the K-pulse shown in Figure 5.10 for the circular loop at $\theta=45^{\circ}, \hat{\theta}$-polarization with no noise. $(k r=0.02 \pi(0.02 \pi) 4 \pi)$. 


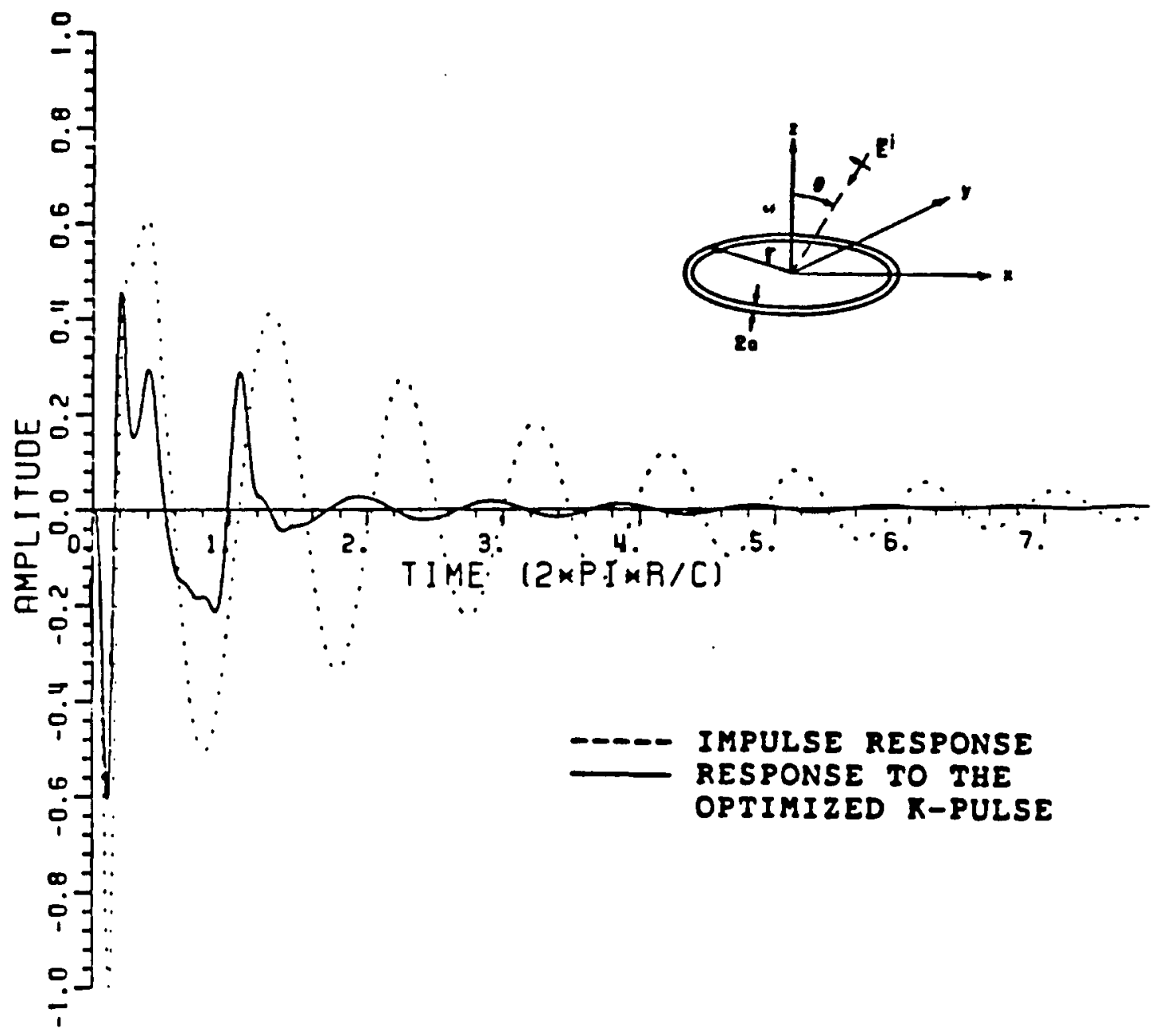

Figure 5.13. Impulse response and response to the $K$-pulse shown in Figure 5.10 for the circular loop at $\theta=0^{\circ}, \hat{\phi}$-polarization with no noise. $(k r=0.02 \pi(0.02 \pi) 4 \pi)$. 


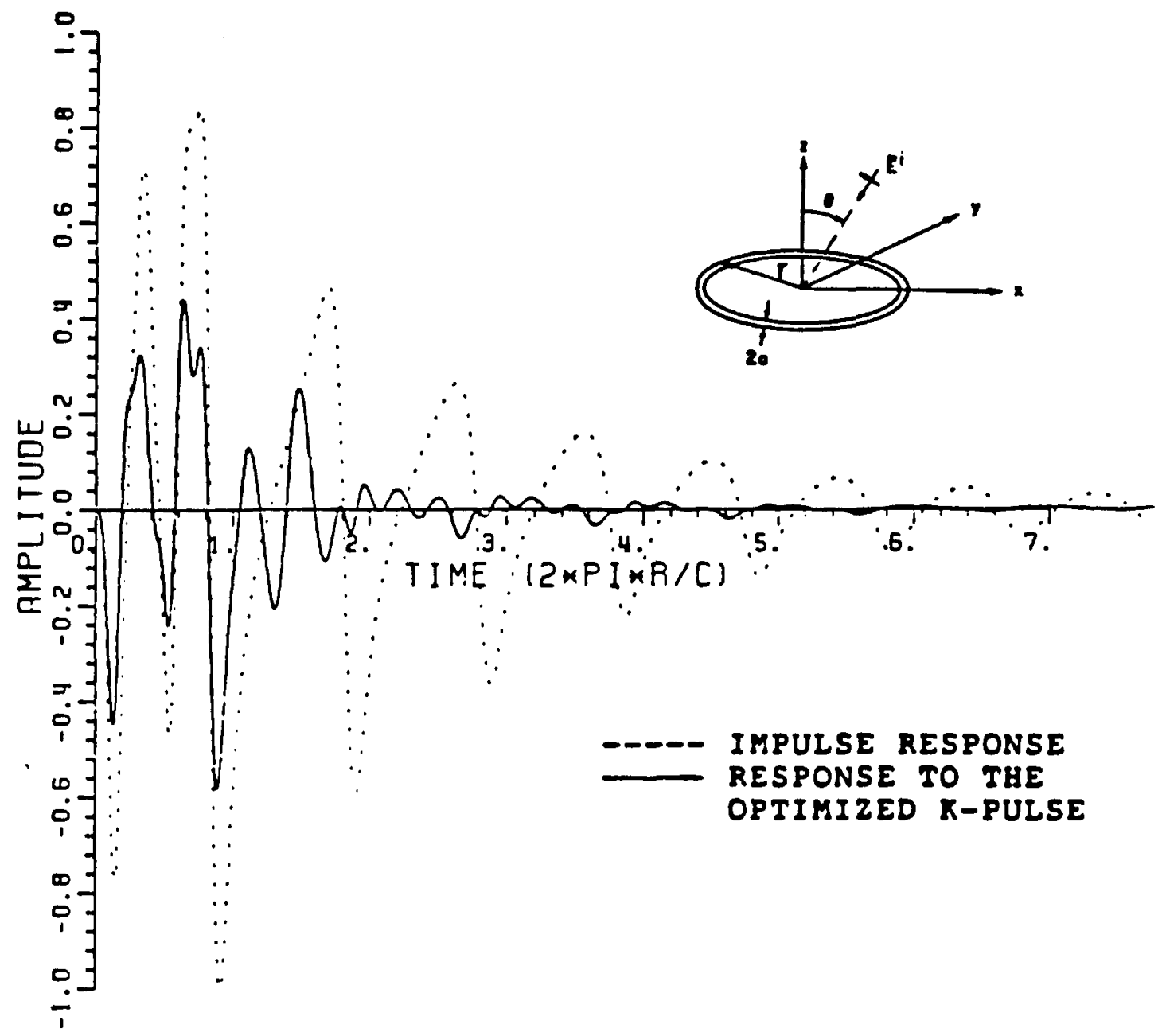

Figure 5.14. Impulse response and response to the $K$-pulse shown in Figure 5.10 for the circular loop at $\theta=45^{\circ}, \hat{\phi}$-polarization with no noise. $(k r=0.02 \pi(0.02 \pi) 4 \pi)$. 


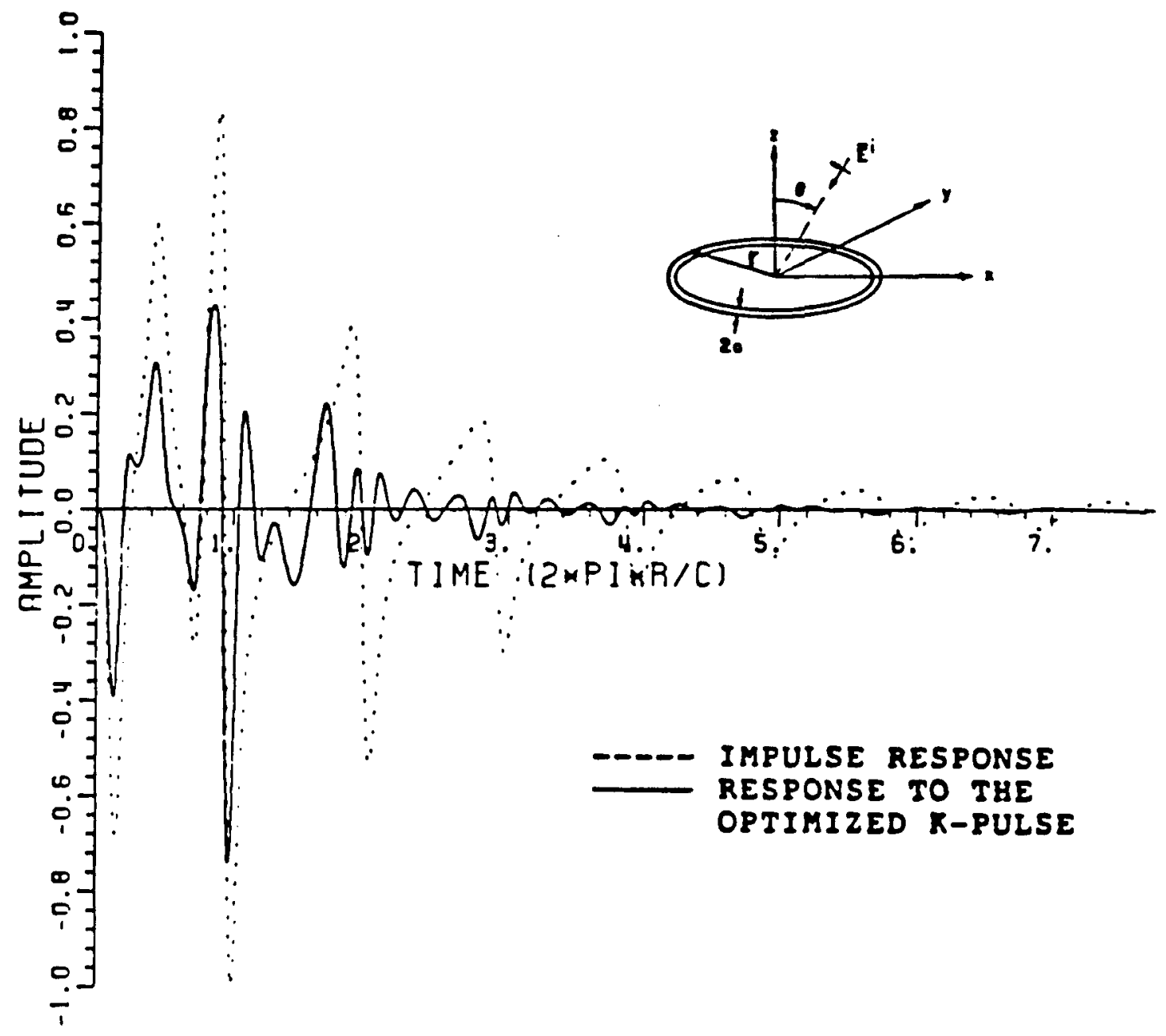

Figure 5.15. Impulse response and response to the $K$-pulse shown in Figure 5.10 for the circular loop at $\theta=90^{\circ}, \phi$-polarization with no noise. $(k r=0.02 \pi(0.02 \pi) 4 \pi)$. 


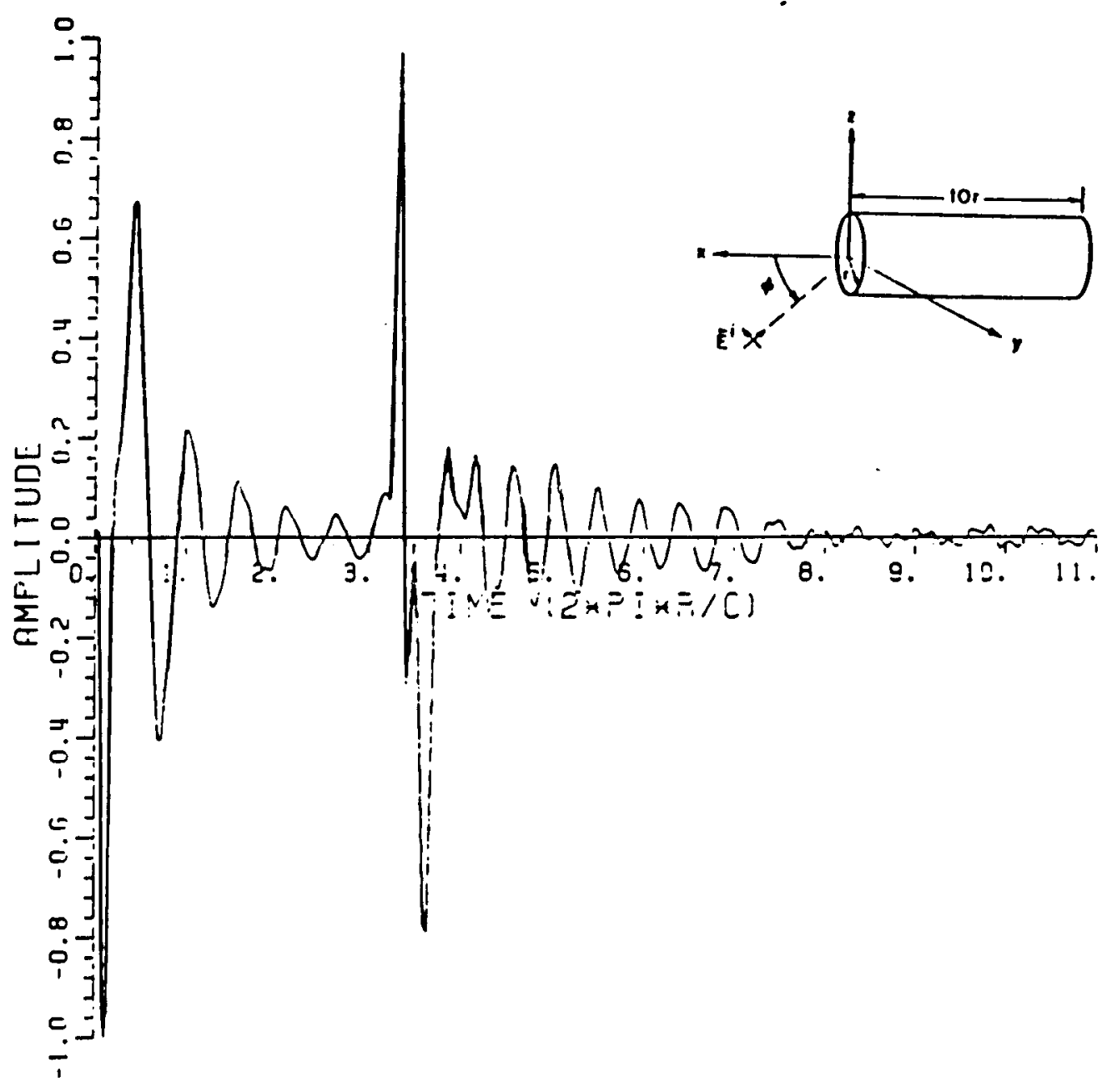

Figure 5.16. Fourier synthesized impulse response of the open ended finite circular waveguide at $\theta=0^{\circ}, \phi=0^{\circ}$, and vertical polarization with $k r=0.00665(0.00665) 12$. 


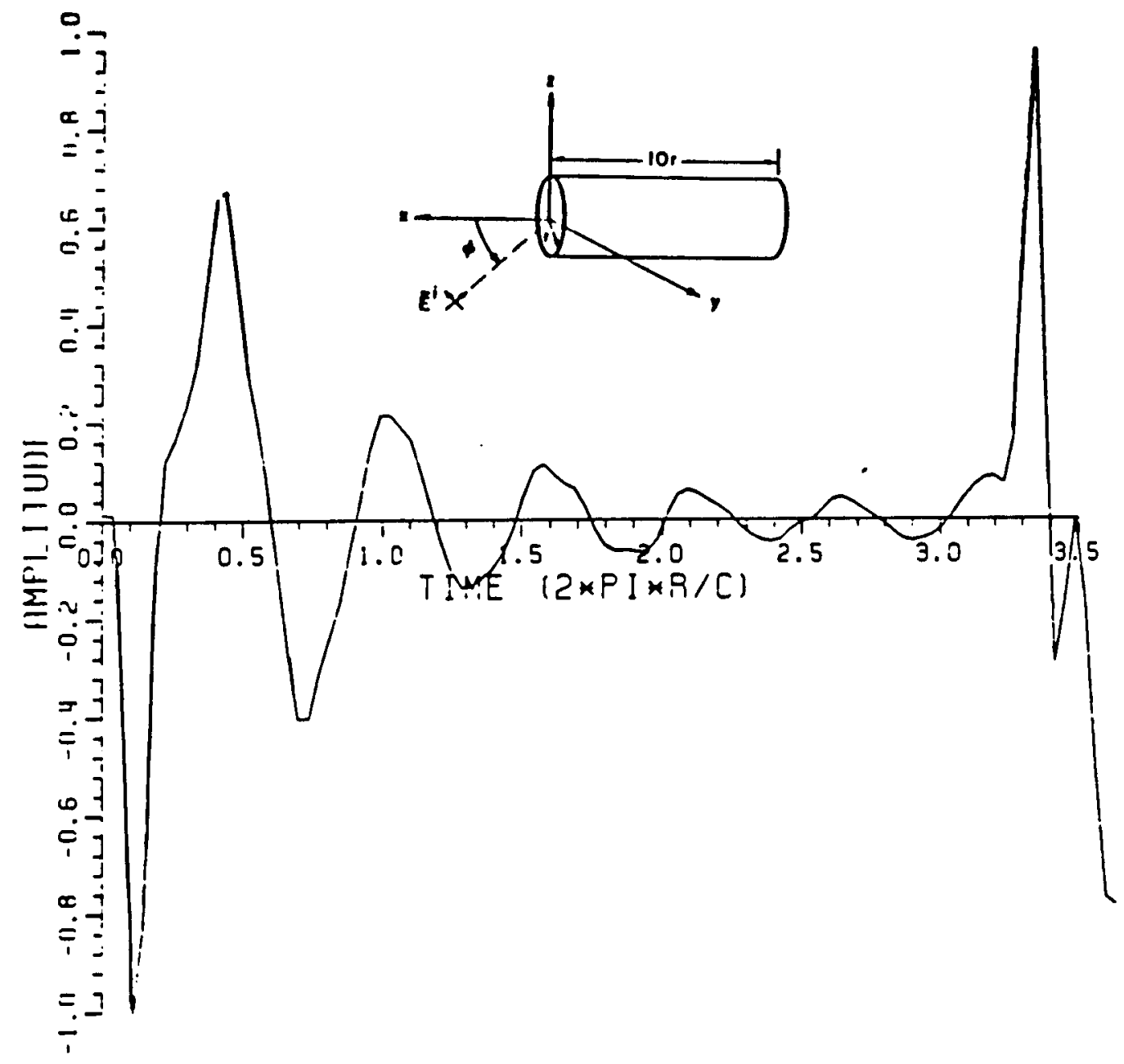

Figure 5.17. Same waveform as in Figure 5.16, except the time scale is given up to $7 \pi r / c$ - equivalent to the Fourier synthesized impulse response of a semi-infinite circular waveguide at $\phi=0^{\circ}, \theta=0^{\circ}$, and vertical polarization with $k r=0.00665(0.00665) 12$. 


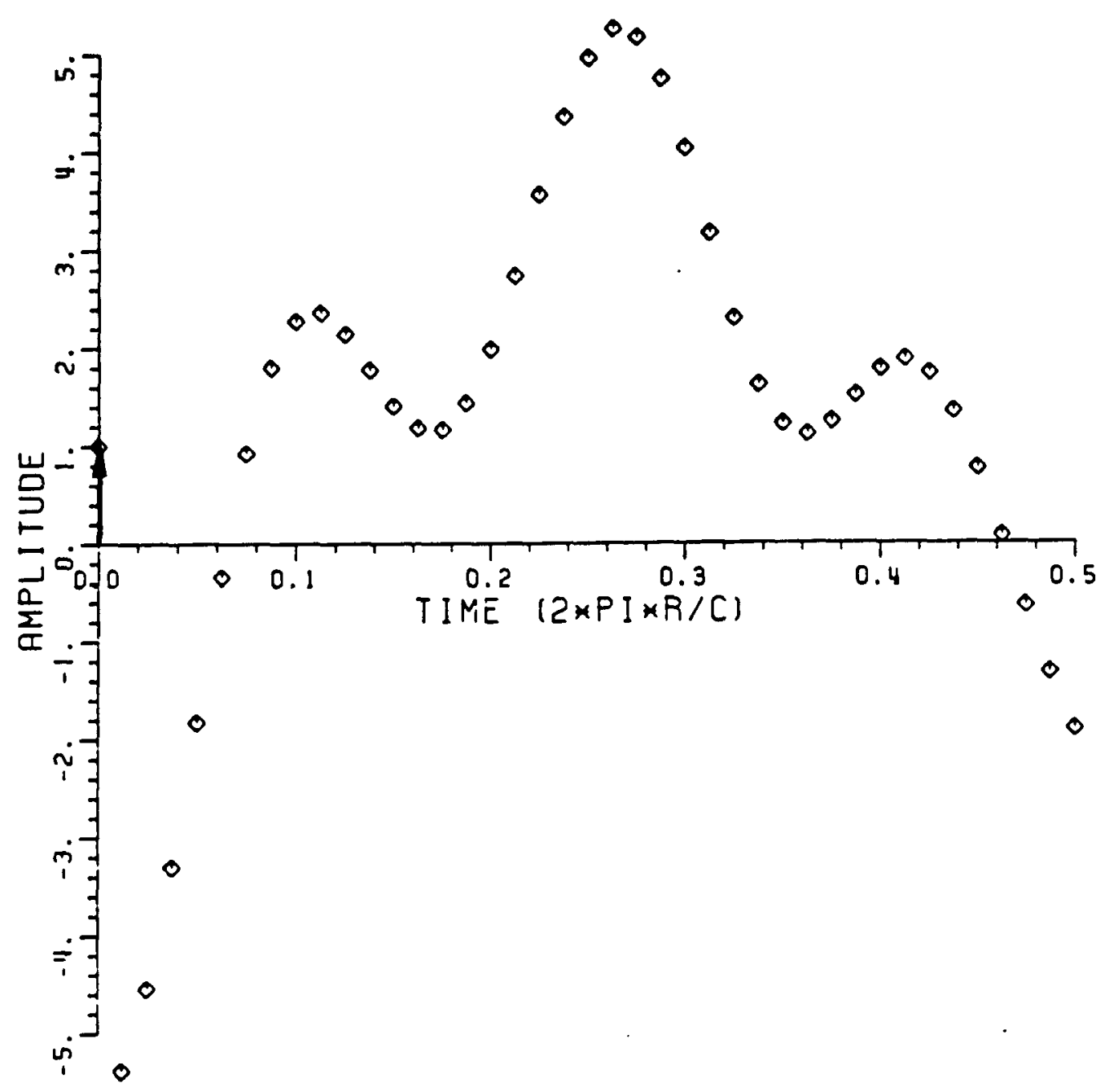

Figure 5.18. K-pulse obtained using the impulse response shown in Figure 5.17. 


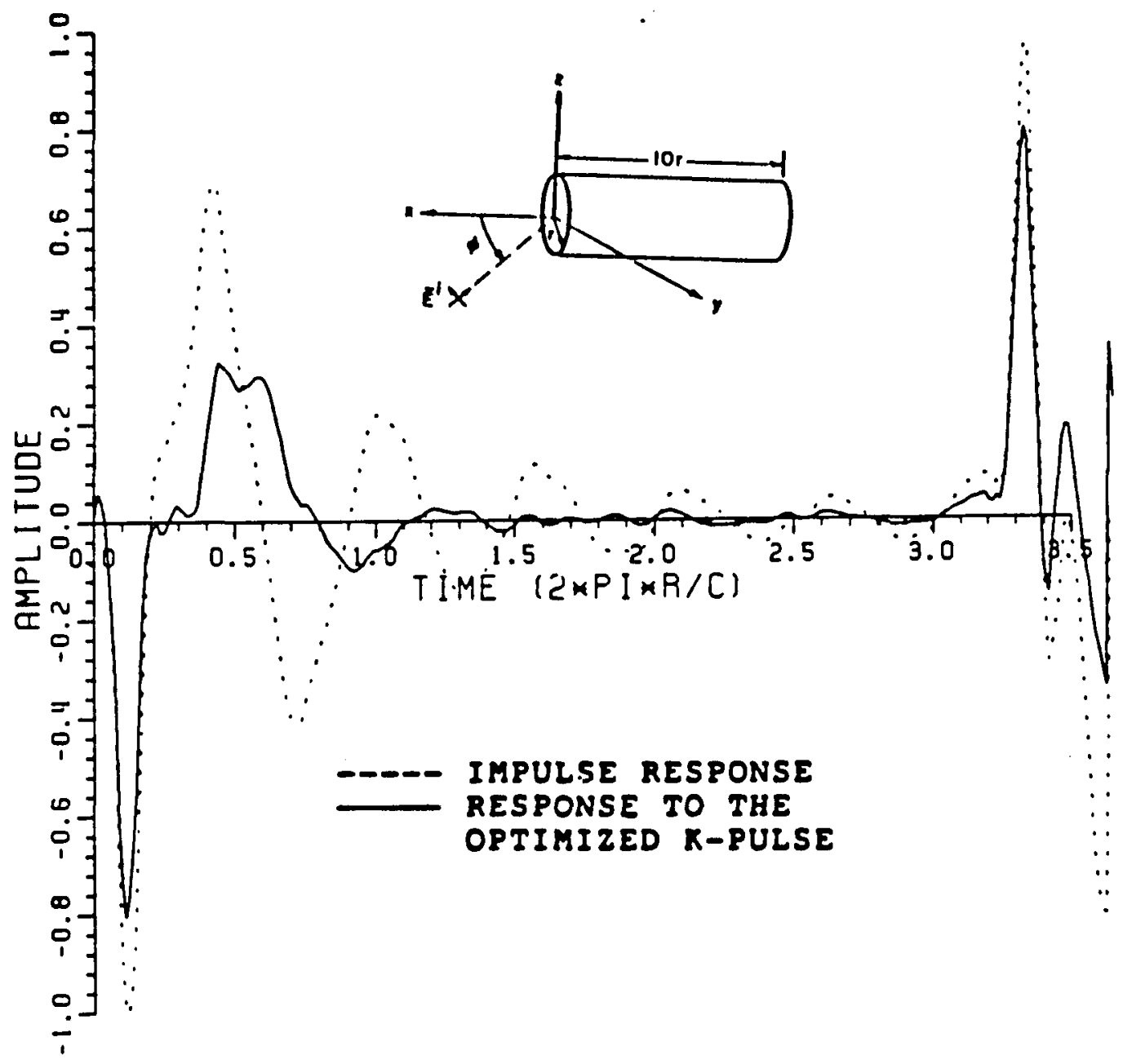

Figure 5.19. Figure 5.17 and response to the $K$-pulse shown in Figure 5.18 for the waveform shown in Figure 5.17. 


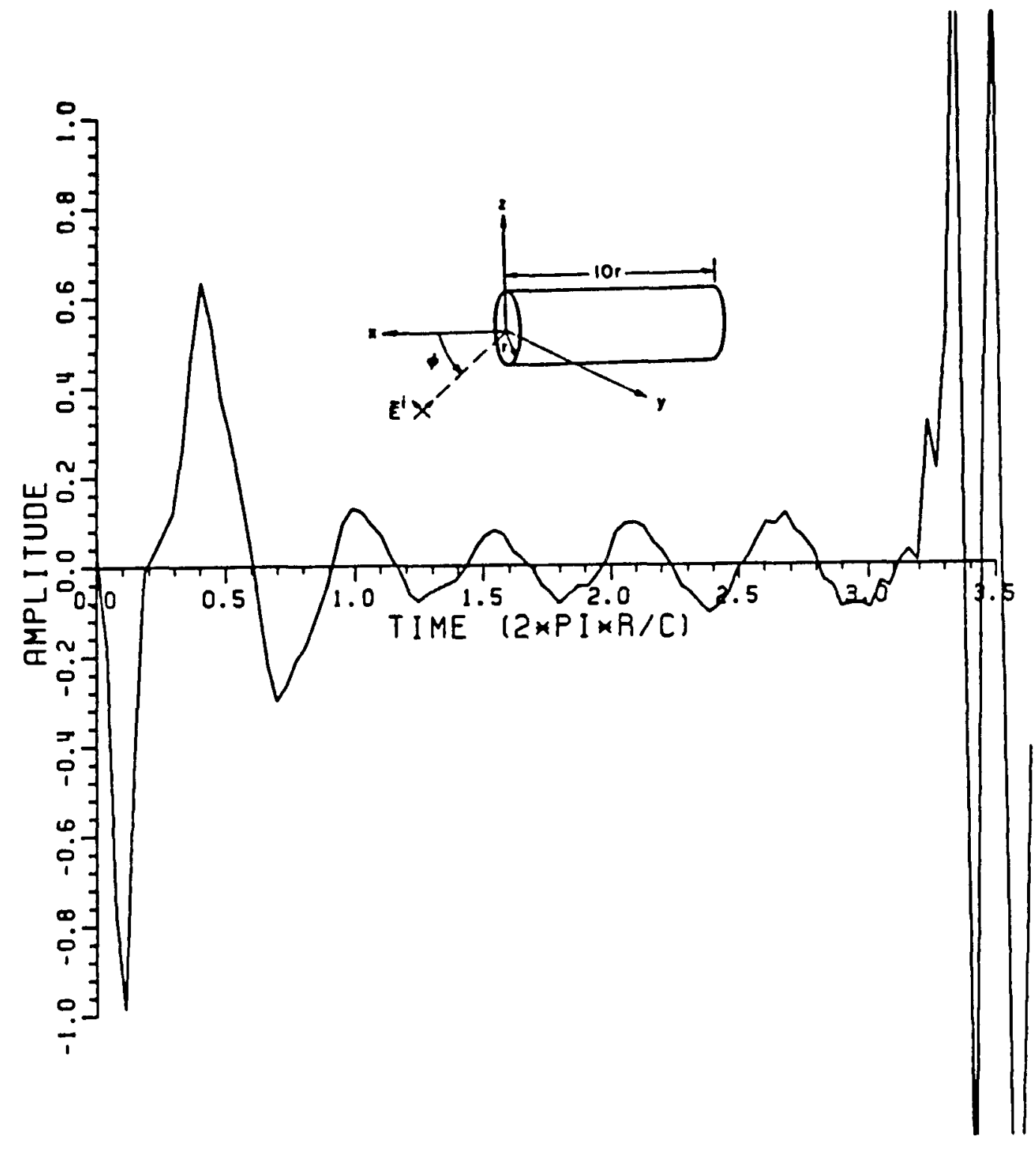

Figure 5.20. Fourier synthesized impulse response of the finite circular waveguide with one open end at $\phi=0^{\circ}$, and one closed end at $\phi=180^{\circ}$. The data are measured at $\phi=0^{\circ}, \theta=0^{\circ}$ and vertical polarization with $k r=0.00665(0.00665) i 2$. 


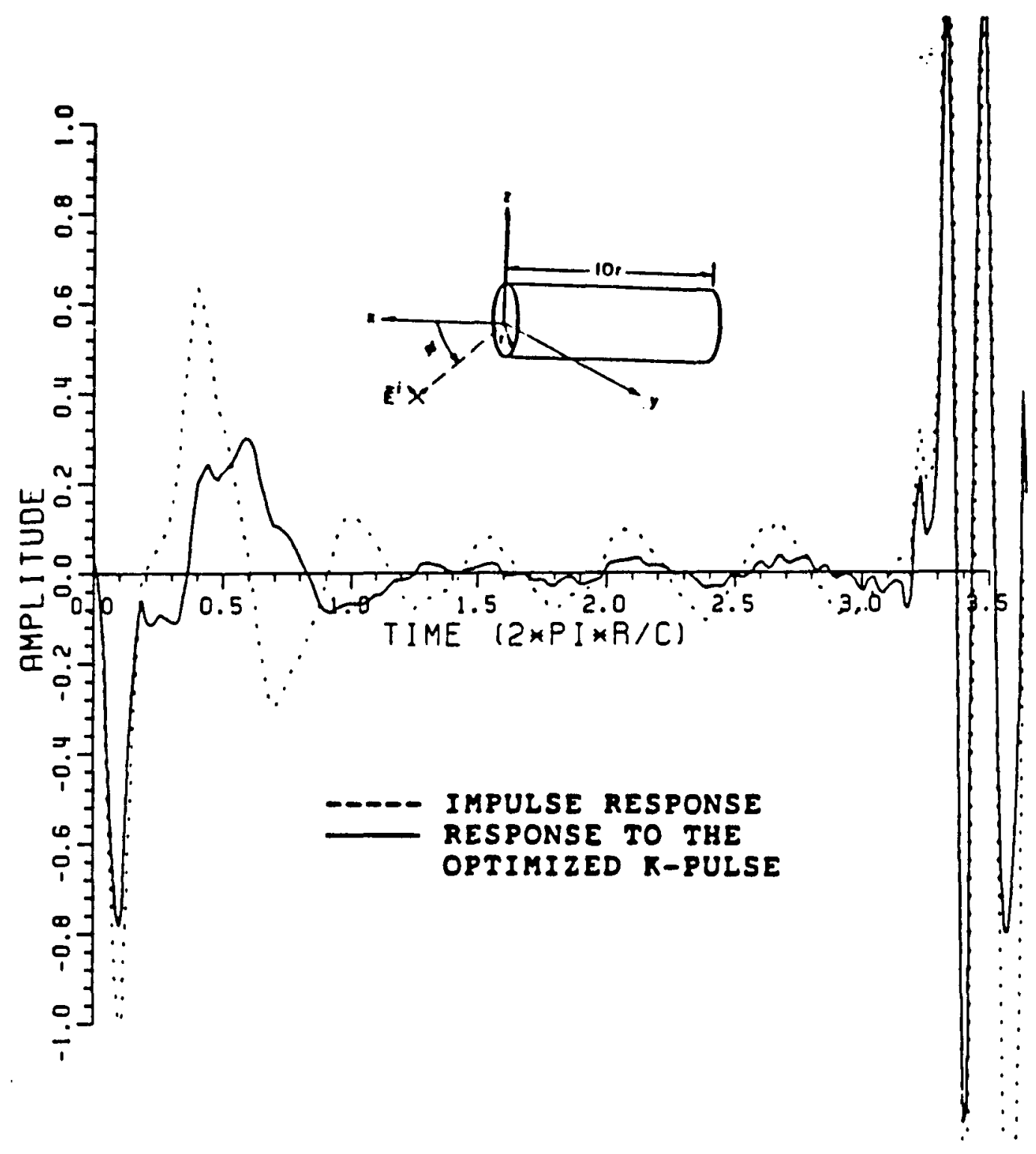

Figure 5.21. Figure 5.20 and response to the $K$-pulse shown in Figure 5.18 for the waveform shown in Figure 5.20. 


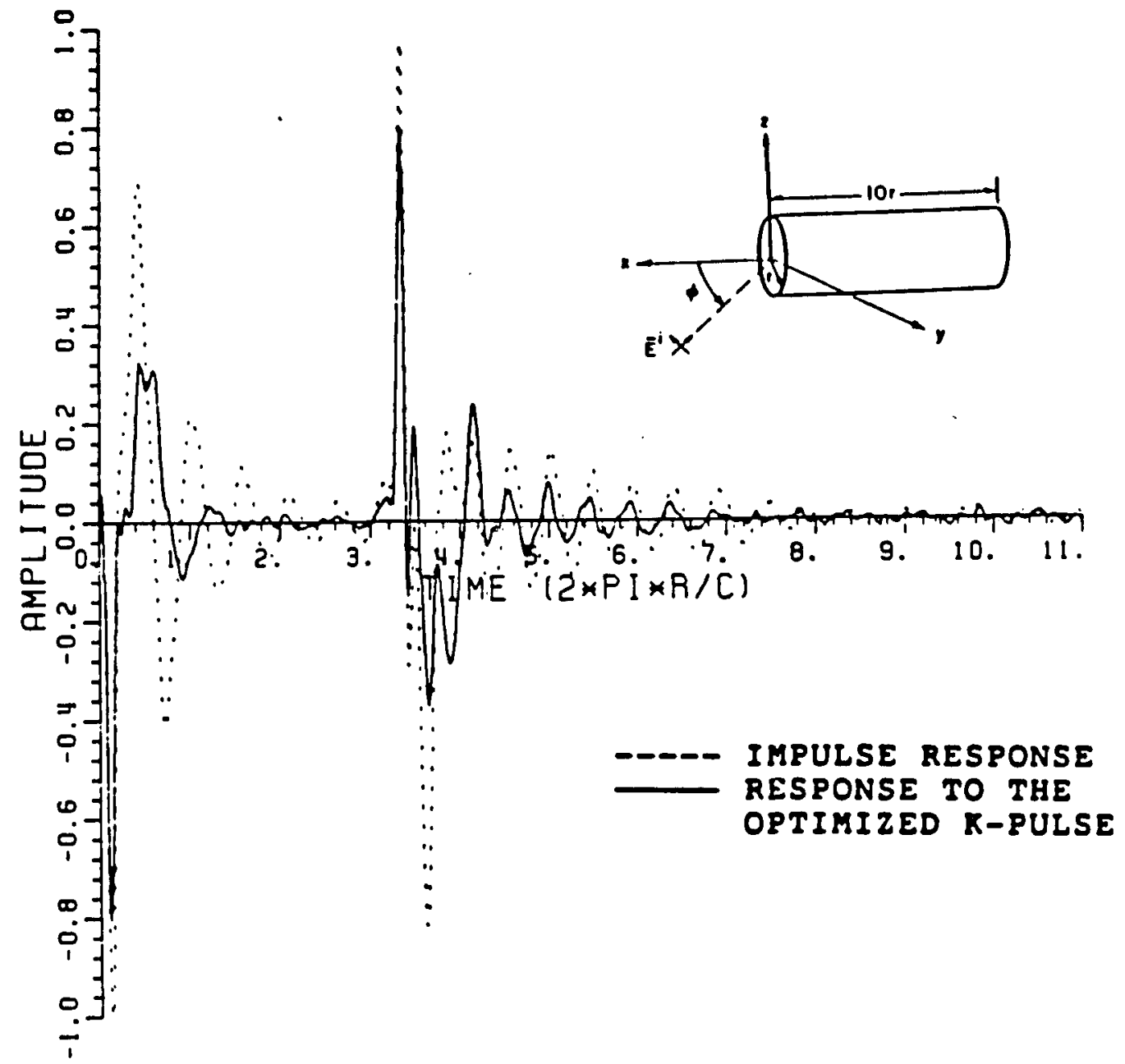

Figure 5.22. Impulse response and response to the $K$-pulse shown in Figure 5.18 for the open ended finite circular waveguide at $\phi=0^{\circ}, \theta=0^{\circ}$ and vertical polarization with $k r=0.00665(0.00665) 12$. 


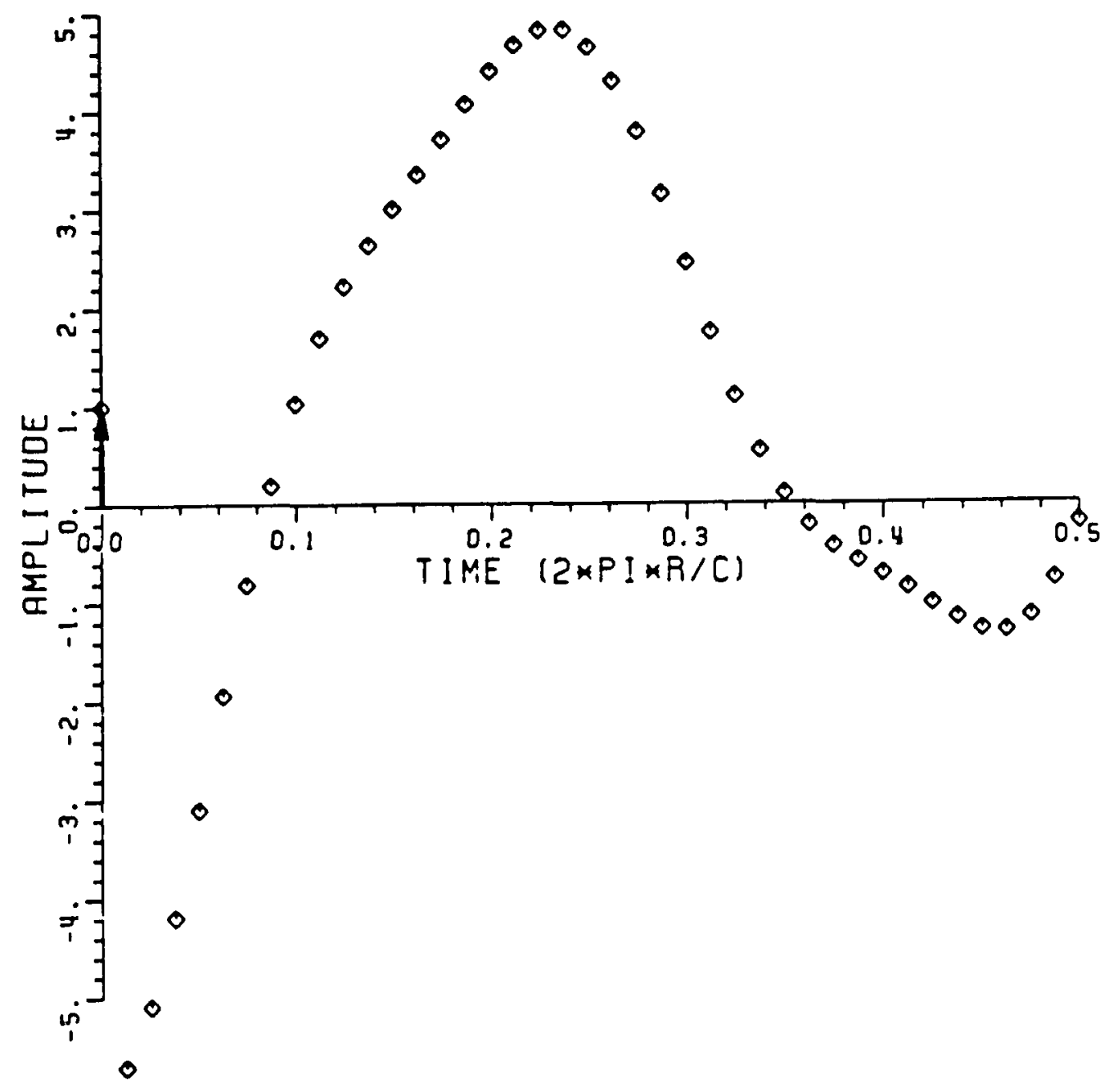

Figure 5.23. K-pulse obtained using the Fourter synthesized impulse response of the open ended finite circular waveguide at $\phi=0^{\circ}, \theta=0^{\circ}$ and vertical polarization with $k r=0.00665(0.00665) 12$. 


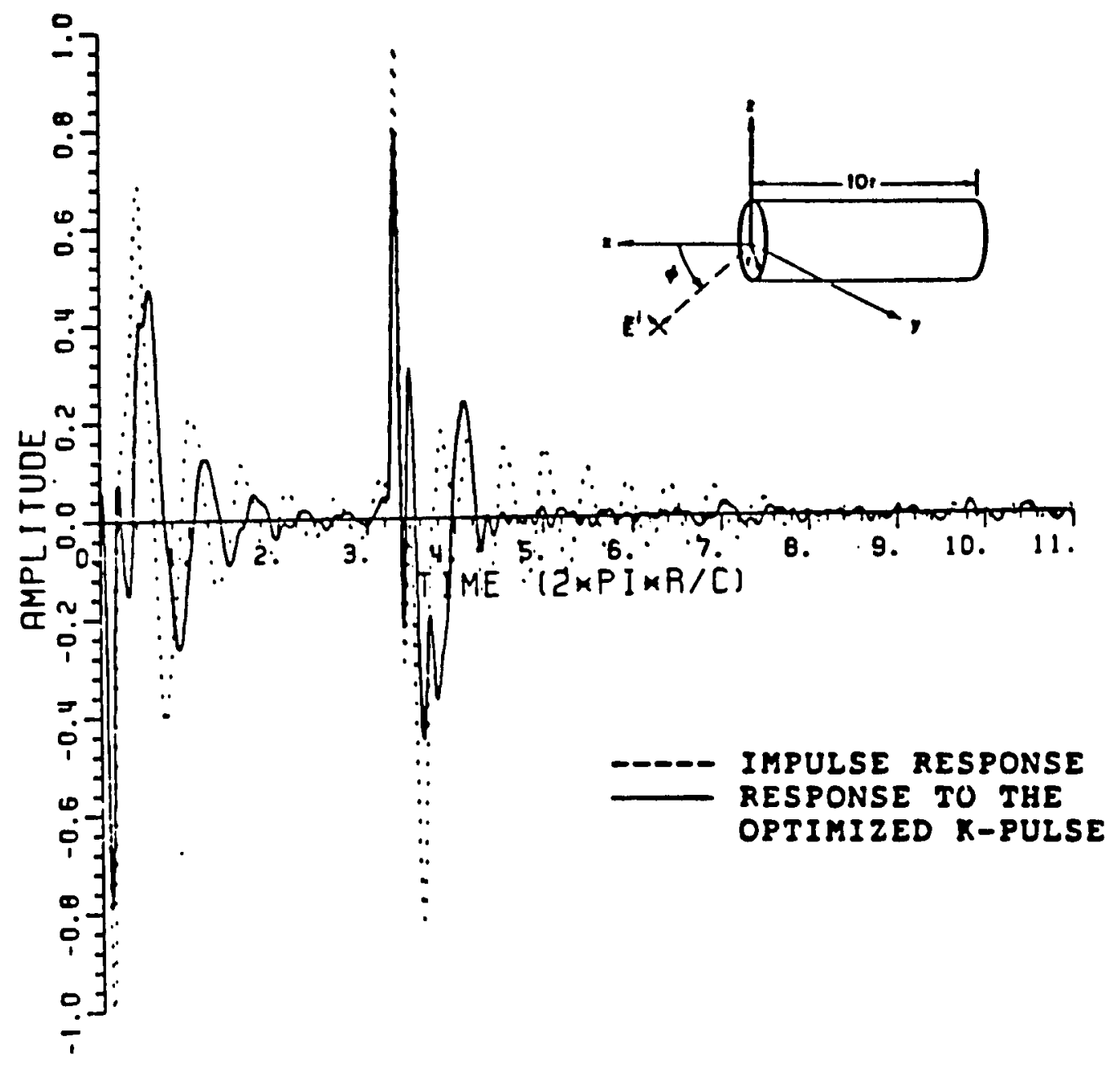

Figure 5.24. Impulse response and response to the $K$-pulse shown in Figure 5.23 for the open ended finite circular waveguide at $\phi=0^{\circ}, \theta=0^{\circ}$ and vertical polarization with $k r=0.00665(0.00665) 12$. 


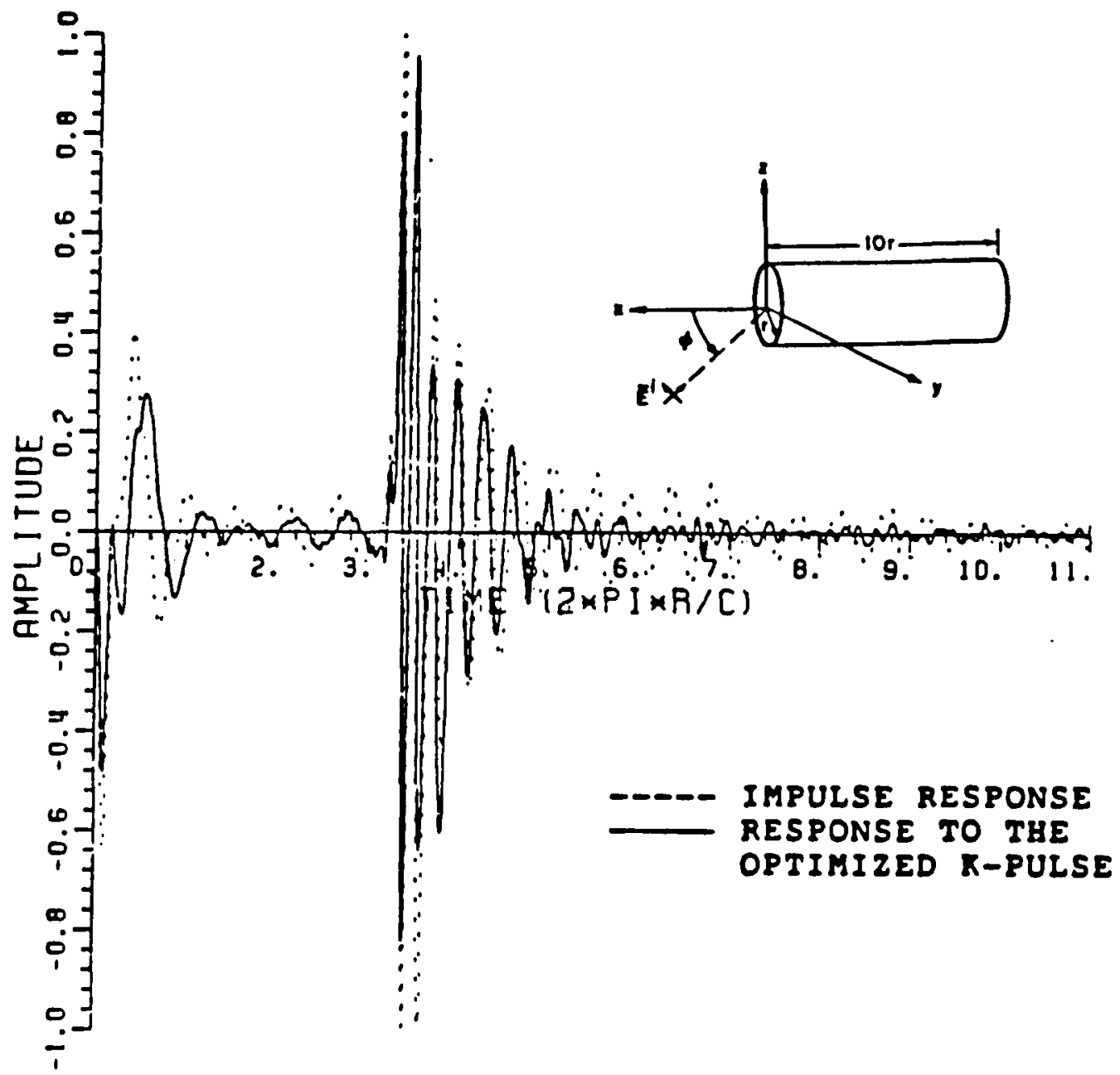

Figure 5.25. Impulse response and response to the $K$-pulse shown in Figure 5.23 for the finite circular waveguide with one open end at $\phi=0^{\circ}$, and one closed end at $\phi=180^{\circ}$. The data are taken at $\phi=0^{\circ}, \theta=0^{\circ}$ and vertical polarization with $k r=0.00665(0.00665) 12$. 


\section{CHAPTER VI}

\section{CONCLIJSIONS AND RECOMMENDATIONS}

This dissertation has presented, for the first time, a method whereby the K-pulse of a target can be approximated without a priori knowledge of some of the complex natural resonances of the target. The only knowledge required is the impulse response of the target efther obtained directiy in the time domain or as multiple frequency samples of the scattered field. In the latter case the impulse response is obtained via Fourier synthesis. Even though the impulse response will be band-limited in practice, it does not affect the generated K-pulse. It is felt that the material in this dissertation represents a major advance in that for the first time, as demonstrated herein, it is possible to obtain an estimate of the K-pulse of an arbitrary target from measured scattering data. This means that measured data from a broadband reflectivity facility such as the compact range at the Electroscience Laboratory can be employed directly to obtain an estimate of the K-pulse.

The model used to obtain an estimate of the K-pulse is particularly simple - only weighted delta functions are used. The convolution involving this $K$-pulse model and the impulse response of the target is thus simple. These delta functions in the $K$-pulse model can be considered as samples of a continuous function provided the sampling criterion is satisfied. There is no evidence at this time that a more 
complicated model would improve the estimate of the K-pulse. Besides, the fact that the delta functions can represent samples of a continuous function means a lot of the function can be modelled. Therefore, this model is also quite general. The unknown wefghts in the K-pulse model are obtained by minimizing the energy which, in time, lies beyond the K-pulse response duration. The optimization is performed by available computer programs. For this reason, time and financial considerations also dictate the need for simplicity in the model.

Once the $K$-pulse estimate has been obtained, the dominant complex natural resonances of the target can also be determined from this K-pulse estimate. This is possible because the whole theory of this dissertation is based on minimizing the energy due to the complex natural resonances of the target. This is also a new numerical procedure to extract the complex natural resonances of a target. Target identification can be performed using these complex natural resonances. It may well be that in the future the $K$-pulse and the target responses to the K-pulse will be viewed as the optimum canonical waveforms of the target, like the impulse, step and ramp responses now. This dissertation presents the K-pulse estimates, the responses to the approximate K-pulse and the complex natural resonances for the following geometries: 1) a plane wave normally incident on a grounded dielectric slab, 2) a thin conducting straight wire, 3) a simulated low $Q$ model, 4) a thin conducting circular loop, 5) a conducting sphere, 6) a conducting circular disc, 7) two conducting circular waveguides - one with open ends, and the other with one closed end and one open end. The results 
of the first six targets show that the imaginary parts of the complex natural resonances are always very close to the true values, but only the real parts of the more dominant complex natural resonances are close to the theoretical values.

The duration of the $k$-pulse must be known and chosen reasonably close, in order to obtain an approximate $K$-pulse which has a close resemblance to the true $K$-pulse. For simple geometries, the $K$-pulse duration is chosen to be the circumnavigational path. For complicated geometries, the circumnavigational path is not very obvious. A more general way to estimate the $K$-pulse duration is required. By using a conservative estimate for the K-pulse duration, which is usually the periodicity of the damped sinusoid at late time of the impulse response, the zero strings of $k$-pulses with different durations are compared. The Imaginary parts of similar zeros from the $K$-pulses are used to obtain a better approximation to the K-pulse duration; namely, the difference between neighbouring common zeros. This approach seems to give a very close value to the true $K$-pulse duration for high $Q$ targets, and also low $Q$ structures whose late time responses possess substantial pole contributions. For other low $Q$ structures, the method sti11 estimates the K-pulse duration reasonably well if the duration of the K-pulse response is known reasonably well.

All in all, this dissertation has presented a very simple and general technique to get an estimate of the K-pulse for an arbitrary target using the impulse response of the target. The method works best with high $Q$ structures but low $Q$ structures can also be treated. Now 
that a general method to obtain the $K$-pulses is available, the applications of the K-pulse can be further investigated. After a catalog of K-pulses is obtained for some reallstic target models, a practical target discrimination system can be built to see one of the practical applications of the K-pulse concept. At the Electroscience Laboratory, the measurement performed with the compact range is in the frequency domain. The method described in this dissertation, operates on the Fourier synthesized frequency data or in the time domain. The Fourier synthesis always adds some numerical errors and human interpretations. A method to determine the $\mathrm{K}$-pulse of the target directly from the frequency domain is very much preferred, as the data are already acquired in the frequency domain. All these are some of the areas which can be further studied. 


\section{APPENDIX A}

\section{PROCEDURES TO ESTIMATE THE K-PULSE}

In this appendix, the conducting circular loop is used as a target example to illustrate the steps and indicators in arriving at the K-pulse shown in Figure 3.17. The duration of K-pulse $(T)$ is assumed to be known (see Chapter IV, otherwise). For the 100p, the duration of the $K$-pulse is $2 \pi r / c$, where $r$ is the radius of the loop. To begin the process, an angle with a particular polarization at which the target will exhibit most of its natural resonant behaviour is chosen. With the loop, $\theta=45^{\circ}$ at $\hat{\theta}$-polarization is chosen. Either the impulse response or the frequency response is measured or generated. If the frequency response is measured or generated, then the synthesized impulse response is obtained via fast Fourier transiorm. It is better to start the process with a smaller bandwidth to reduce $N$, the number of samples required to approximate the continuous portion of the $K$-pulse. If $N$ is small, the computation time is also smaller. Naturally, the sampling of the impulse response must satisfy the sampling theorem in order to approximate the true impulse response. The impulse response shown in Figure A.1 has a bandwidth of $k r=0.02 \pi(0.02 \pi) 4 \pi$.

The duration of the impulse response is theoretically infinite. For other than very high $Q$ targets, the impulse response damps quickly to negligible values. The practical duration of the impulse response should be long enough to include a few "cycles" of contributions from tive poles. Figure A.1 shows the duration of the impulse response of the 


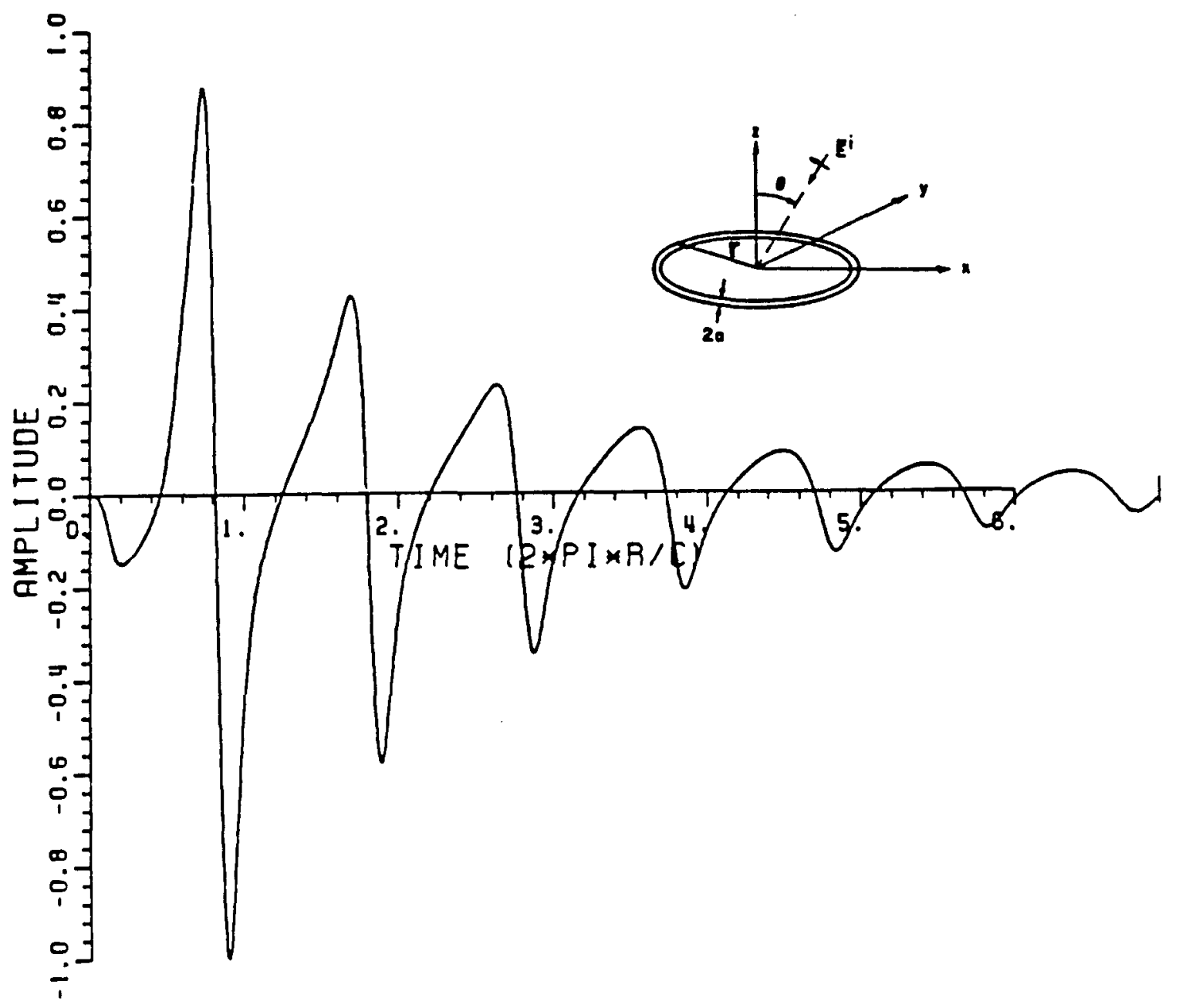

Figure A.1. Fourier synthesized impulse response of the conducting circular loop at $\theta=45^{\circ}, \hat{\theta}$-polarization. $(k r=0.02 \pi(0.02 \pi) 4 \pi)$. 
loop at $\theta=45^{\circ}, \hat{\theta}$-polarization to be $12 \pi r / c$. This includes five "cycles". Since the loop is a high Q structure, experience has shown that the duration of the K-pulse response duration $\left(T_{R}\right)$ does not have to be known exactly. This is because the later cycles would also contain large contributions from the lower order poles. The range of time over which the energy of the $K$-pulse response is to be minimized can be any number of "cycles". For this example, the duration of the K-pulse response $\left(T_{R}\right)$ is chosen to be $6 \pi r / C$; the cutoff time for integration $\left(T_{C}\right)$ is $10 \pi r / C$; and the number of samples $(N)$ to represent the continuous portion of the $k$-pulse $\left[k_{c}(t)\right]$ is 50 . The choice of $N=50$ seems to be a good starting value. It gives a reasonable approximation to $k_{c}(t)$ and yet does not take too much cpu time before the bottom of the cost function is found. The choice of $N$ cannot be too small for then it violates the sampling criterion for the impulse response. The computer program will do the optimization with the sampling rate dictated by the K-pulse. In other words, the size of the matrix $\Phi$ in Equation (2.13) is controlled by the user. Also if the number of samples is too small, then the criterion for straight line interpolation may be violated. A good rule of thumb from experience is to choose the sampling rate at least four times the Nyquist rate.

After performing a user-specified number of searching steps, the program will prompt the option of plotting the K-pulse and the response to the K-pulse. It is advisable at this time to check for jump discontinuities in $k_{c}(t)$ and energy reduction in the $k$-pulse response. If the impulse response has no jump discontinuities, there is no reason 
why there should be jump discontinuities in $k_{c}(t)$. If the energy outside the $K$-pulse response is not less than the energy in the impulse response, the program is not going in the down-hill direction. Either of these conditions, $i$. e., jump discontinuities in $k_{c}(t)$ or no energy reduction in the $K$-pulse response, may indicate that the choices of the K-pulse duration $(T)$, the $K$-pulse response duration $\left(T_{R}\right)$, the cutoff time of the integration $\left(T_{C}\right)$, or the number of samples $(N)$ to represent the continuous portion of the $k$-pulse $\left[k_{c}(t)\right]$ may be in error. The geometry of the target in relation to the impulse response should be restudied, such that the proper $T, T_{R}, T_{C}$ or $N$ can be chosen. The optimization process is then repeated.

In the example, fifty steps of Powell-Fletcher search is first specified. Then ten more steps of conjugate gradient and PowellFletcher search are used to do repeated checks for the bottom of the valley. The resulting K-pulse for the loop (Figure A.2) is obtained. The program now prompts the option of writing the coefficients. Then the program prompts the option of convolving the K-pulse obtained with the impulse response of the target at another aspect angle using the sampling rate dictated by the impulse response. Before this option, the computer program uses a sampling rate that is specified by the user to minimize the number of calculations. It is a good idea to convolve with the same impulse response for a further check on energy reduction. The result for the circular loop is shown in Figure A.3.

At this stage, the aspect and polarization independent property of the K-pulse can be checked. Another check is to use a different aspect 


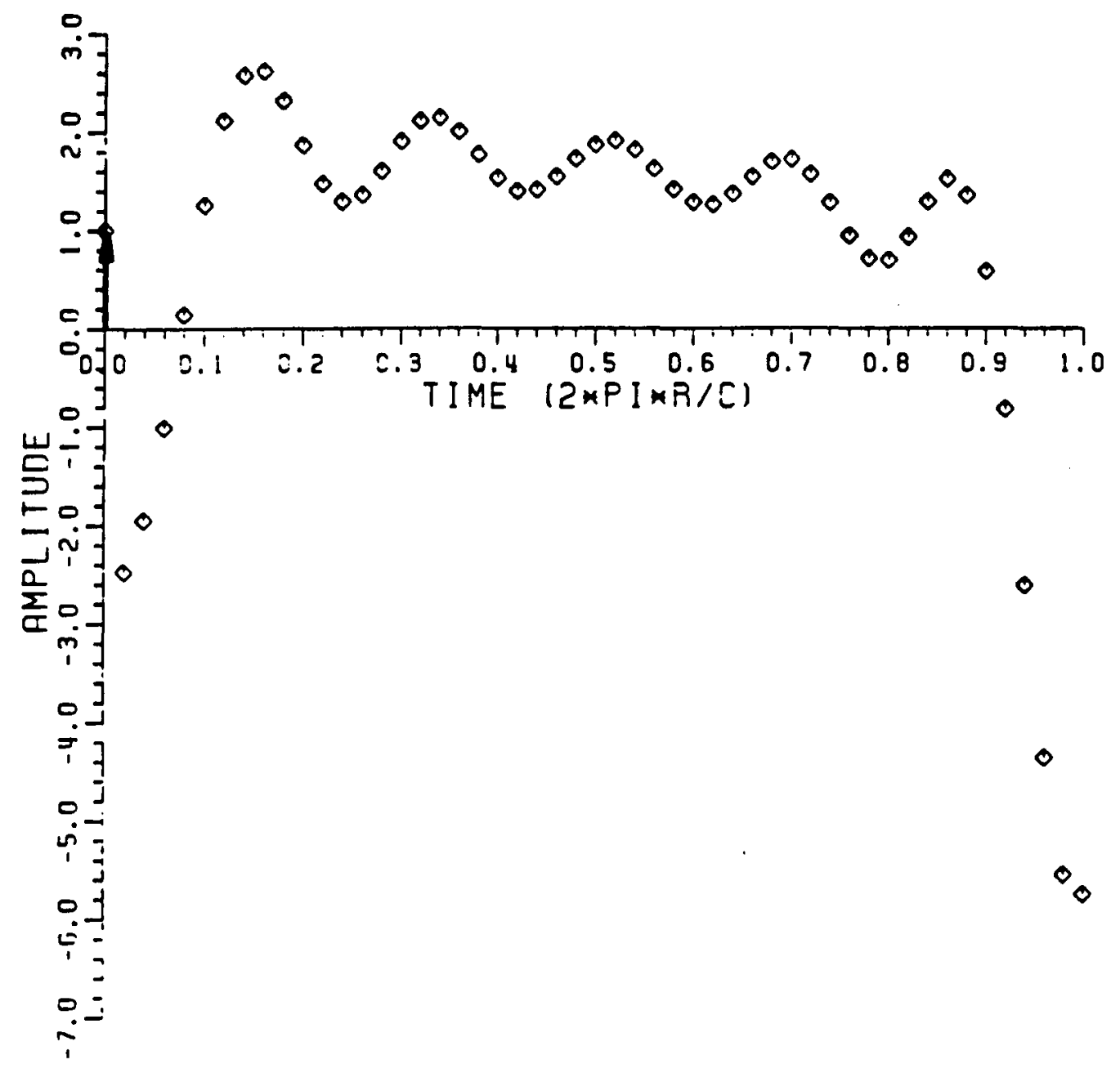

Figure A.2. K-pulse obtained using the Fourier synthesized impulse response of the conducting circular loop at $\theta=45^{\circ}$, $\hat{\theta}$-polarization. $(k r=0.02 \pi(0.02 \pi) 4 \pi)$ The number of samples used to represent the continuous portion of the K-pulse is 50 . 


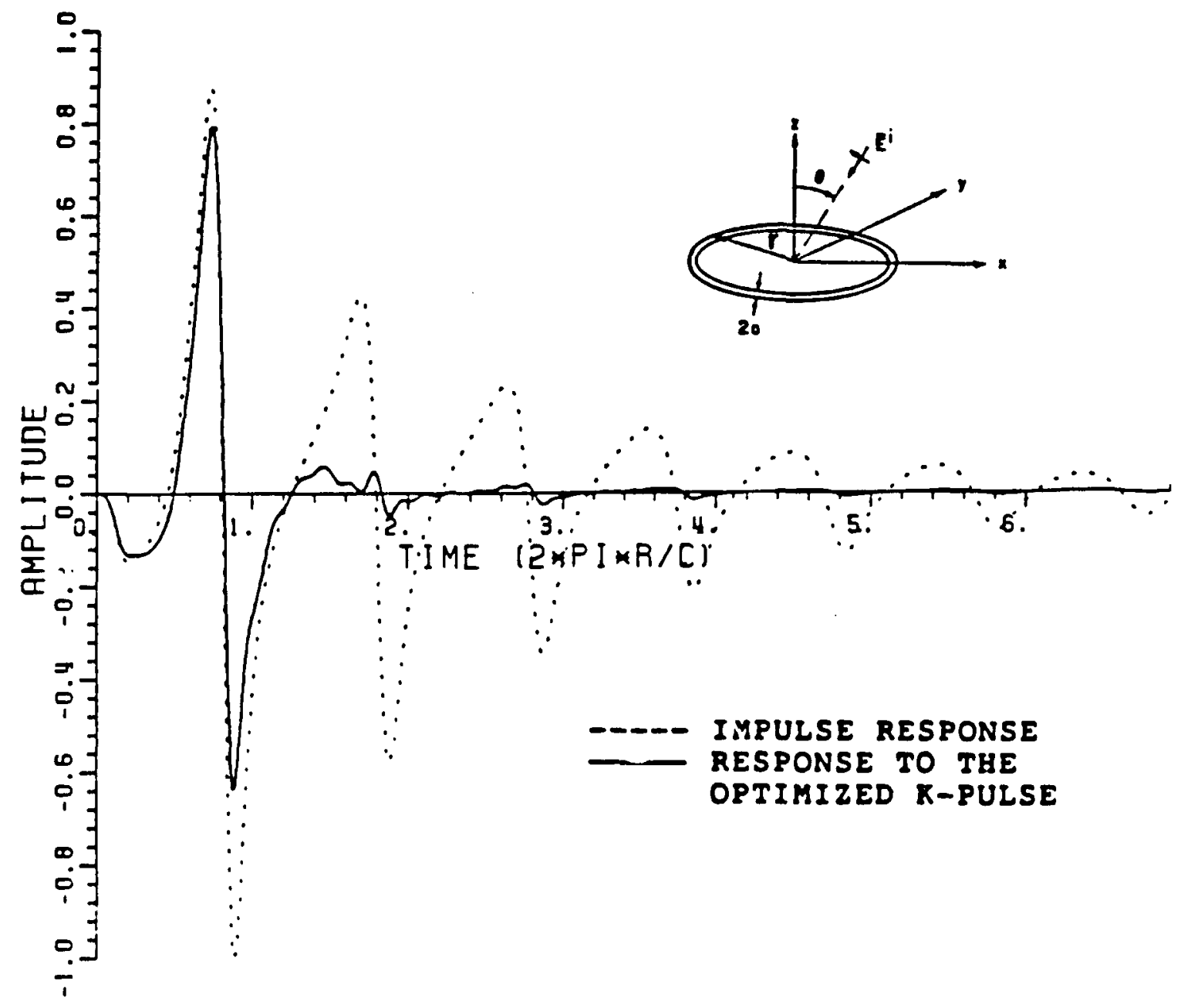

Figure A.3. Impulse response and response to the $K$-pulse shown in Figure $A .2$ for the conducting circular loop at $\theta=45^{\circ}$, $\dot{\theta}$-polarization. $(k r=0.02 \pi(0.02 \pi) 4 \pi)$. 
angle or polarization to check the convergence of the K-pulse, by repeating the optimization process. For this example, the impulse response for the loop at $\theta=45^{\circ}, \hat{\phi}$-polarization is used. The choices of the K-pulse duration $(T)$, K-pulse response duration $\left(T_{R}\right)$, the cutoff time for the integration $\left(T_{C}\right)$ and the number of samples $(N)$ to represent the continuous portion of the $k$-pulse $\left[k_{c}(t)\right]$ are the same as what was used previously. After fifty steps of Powell-Fletcher and twenty-five steps of conjugate gradient search, Figure A.4 is obtained for the K-pulse. The response to the K-pulse is shown in Flgure A.5. The K-pulses shown in Figures A.2 and A.4 are nearly identical. This will only be true, if similar pole strings are excited for the two differnt impulse responses of a high $Q$ target. Since the size of the matrix ( $\Phi)$ is $50 \times 50$, the cpu time for these steps is very short. Fifty steps of searching probably takes less than one minute of cpu time.

The number of samples (N) can be increased to improve the accuracy of the samples of $k_{c}(t)$. For the example, $N$ is doubled and the K-pulse response duration $\left(T_{R}\right)$ is reduced to $5 \pi r / c$. The impulse response used is the first one which is at $\theta=45^{\circ}$, and $\hat{\theta}$-polarized. After ten steps of Powell-Fletcher and conjugate gradient search, Figures 3.17 and 3.18 are obtained. The K-pulses shown in Figures A.2 and 3.17 do not show a drastic change in shape. The aspect and polarization independent properties are checked in Figures 3.19 through 3.21. The energy content after $4 \pi r / C$ in Figure 3.18 is less than that shown in Figure A.3. With a further reduction of the natural resonance energy, the poles should be closer to the true poles. Table 3.3 is repeated in Table A.1 for an 


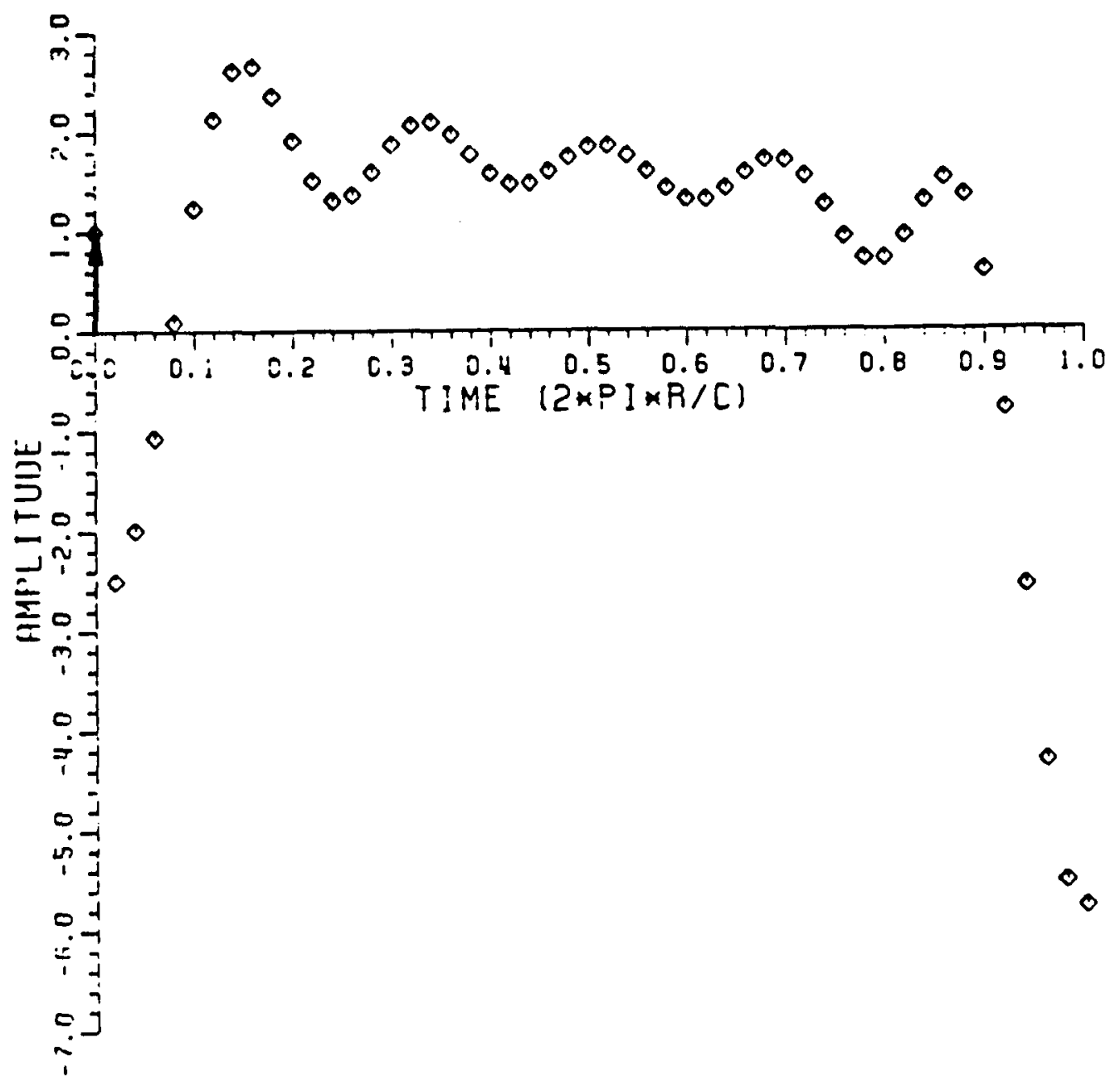

Figure A.4. K-pulse obtained using the Fourier synthesized impulse response of the conducting circular loop at $\theta=45^{\circ}$, $\hat{\phi}$-polarization. $(k r=0.02 \pi(0.02 \pi) 4 \pi)$. The number of samples used to represent the continuous portion of the K-pulse is 50 . 


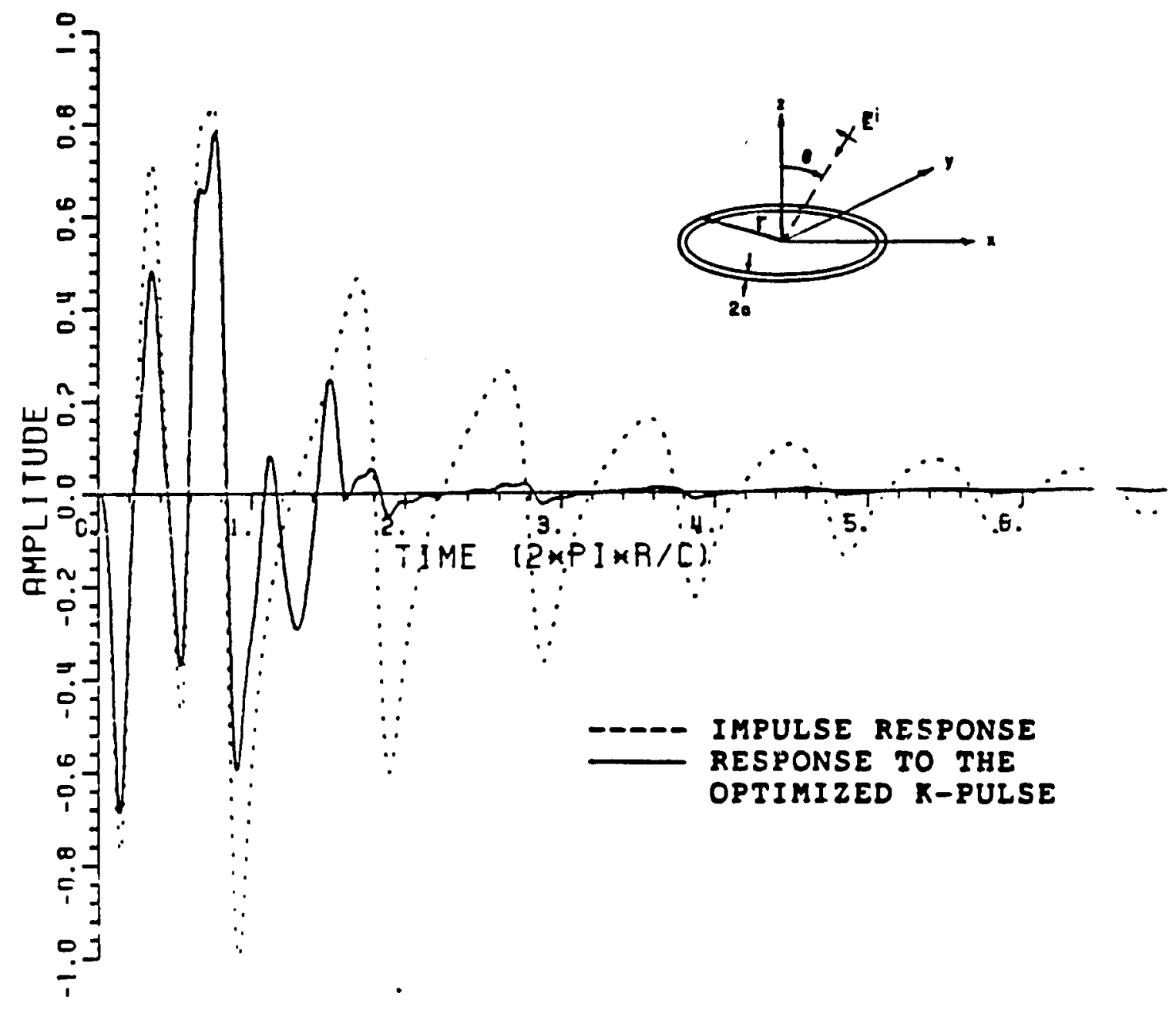

Figure A.5. Impulse response and response to the $K$-pulse shown in Figure A.5 for the conducting circular loop at $\theta=45^{\circ}$, $\hat{\phi}$-polarization. $(k r=0.02 \pi(0.02 \pi) 4 \pi)$. 
easy comparison. Table A.1 lists the first ten poles in the upper left-half plane for the circular loop. The poles are indeed approaching the true poles. The process of increasing $\mathrm{N}$ can be repeated until the sampling of the $K$-pulse is the same as in the impulse response, but the cost of the computer time will increase. The bandwidth of the impulse responses can also be increased to give a more accurate estimation of the K-pulse. There is obviously a trade-off between K-pulse accuracy and computer costs. The application of the K-pulse will ultimately dictate the necessary accuracy. 
Table A.1

A Comparison of the First Ten Exact Poles and Estimated Zeros in the s-domain (Upper Left Half Plane) for the Conducting Thin Circular Loop

$\begin{array}{lccc} & \text { Exact Poles } & \frac{\text { Estimated Zeros }}{(N=100)} & \frac{\text { Estimated Zeros }}{(N=50)} \\ \text { 1) } & -0.423+6.509 j & -0.474+6.509 j & -0.510+6.510 j \\ 2) & -0.606+12.871 j & -0.674+12.891 j & -0.717+12.919 j \\ 3) & -0.745+19.211 j & -0.796+19.248 j & -0.868+19.295 j \\ \text { 4) } & -0.862+25.541 j & -0.949+25.560 j & -0.989+25.691 j \\ 5) & -0.965+31.864 j & -1.055+31.892 j & -1.123+32.087 j \\ \text { 6) } & -1.059+38.183 j & -1.318+38.803 j & -1.469+39.015 j \\ 7) & -1.145+44.499 j & -1.548+45.326 j & -1.748+45.439 j \\ 8) & -1.226+50.812 j & -1.786+51.702 j & -1.975+51.759 j \\ 9) & -1.302+57.124 j & -1.977+58.019 j & -2.149+58.060 j \\ \text { 10) } & -1.374+63.434 j & -2.124+64.319 j & -2.289+64.354 j\end{array}$




\section{APPENDIX B}

\section{A TARGET DISCRIMINATION SCHEME}

A similar target discrimination scheme had been discussed by Moffatt [30]. It is now possible, however, to apply the scheme without a priori knowledge of the target poles. From Chapter I, the frequency response $\left[E_{m}(s)\right]$ of a target at a particular aspect angle and polarization is a memorphic function of complex frequency, it can be decomposed into a fraction of two entire functions of exponential type. (see Appendix D)

$$
E_{m}(s)=\frac{F_{m}(s)}{K(s)}
$$

where $F_{m}(s)$ and $K(s)$ are entire functions of exponential type. Since the $K$-pulse response of the target at the $\mathrm{m}^{\text {th }}$ aspect and polarization $\left[F_{m}(s)\right]$ is an entire function of exponential type, its time waveform is time-limited [4]. The simplest way to discriminate targets is to compare the energy content in the late time of the $K$-pulse response. Target discrimination via late time energy was also discussed by Chen [31].

Consider two conducting targets, a thick circular loop and a thin circular loop, and let the radius of the thick wire to the thin wire be $\pi$. If the thin circular loop is present and the hypothesis is the thin circular loop, then convolution of $k(t)$ (Figure 3.17) with the Fourier synthesized or measured impulse responses at any aspect angle and 
polarization will have very little energy content in the late time

(Figures 3.18 through 3.21). If the thick circular loop is present and the guess is still the thin circular loop, then the convolution of $k(t)$ (Figure 3.17), the guessed target K-pulse, with the Fourier synthesized or measured impulse responses at any aspect angle or polarization will exhibit large energy content in the late time. This is shown, in Figures B.1 and B.2, which are the responses of the thick loop to the K-pulse of the thin loop at $\theta=30^{\circ}$ and $60^{\circ}$, respectively. Both impulse responses are $\hat{\theta}$-polarized and have a bandwidth of $k r=0.042(0.042) 4.2$. The energy content in the late portion of the response is large; therefore, the hypothesis of the thin loop is wrong.

Using the same idea, a target discrimination scheme can be derived. The avallability of K-pulses for a set of targets is assumed in the following discussion. A parallel system is set up as in Figure B.3. A target's broadband Fourier synthesized or measured impulse response $\left[e_{m}^{n}(t)\right]$ is given and the question of which one in the set does this impulse response belong to is raised. This target's impulse response is convolved with all the K-pulses individually but simultaneously. The energy content in the late time is compared among all the K-pulse responses. The choice of the most probable target is the one having the least energy content in the late time. Since late time is not well defined, the operation can employ the elimination process. This is done by comparing the energy content from the cutoff time of integration $\left(T_{C}\right)$ to the K-pulse response duration $\left(T_{R}\right)$ backwards in reasonable increments. Stepping hackwards one increment at a time, the higher 


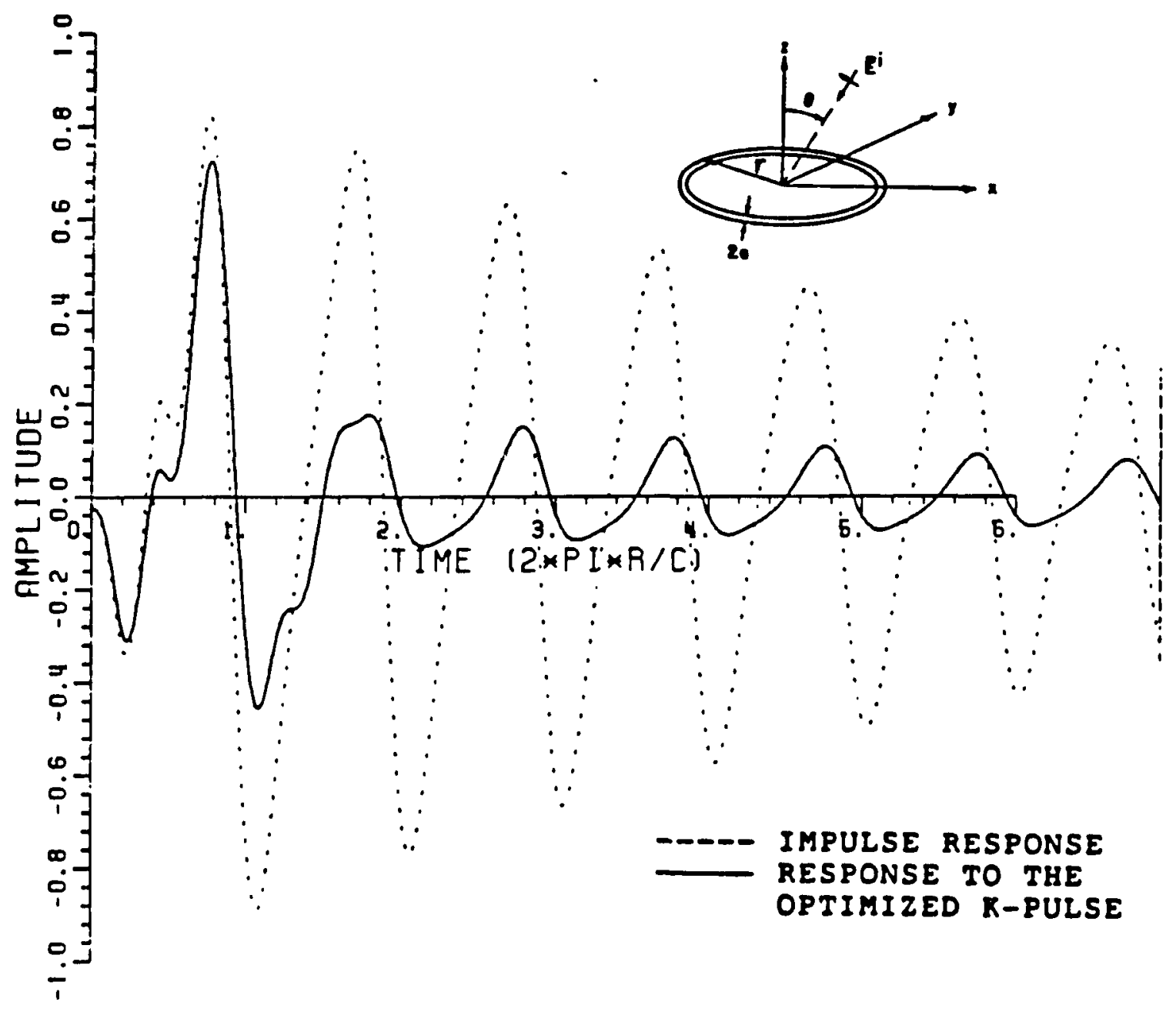

Figure B.1. Impulse response and response to the $K$-pulse shown in Figure 3.17 for the thicker conducting circular loop at $\theta=30^{\circ}, \hat{\theta}$-polarization with $\mathrm{kr}=0.042(0.042) 4.2$. 


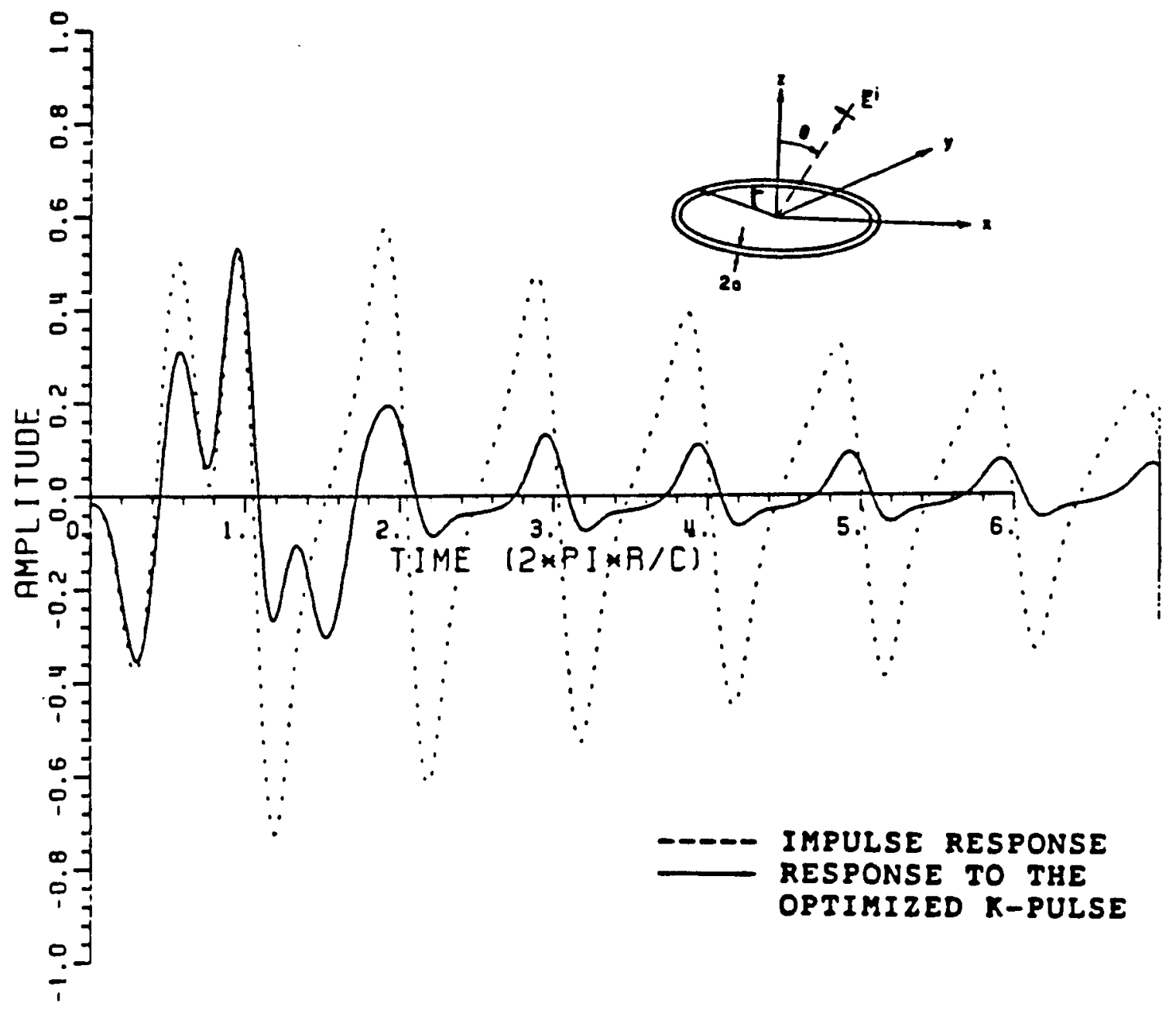

Figure B.2. Impulse response and response to the $K$-pulse shown in Figure 3.17 for the thicker conducting circular loop at $\theta=60^{\circ}, \hat{\theta}$-polarization with $\mathrm{kr}=0.042(0.042) 4.2$. 


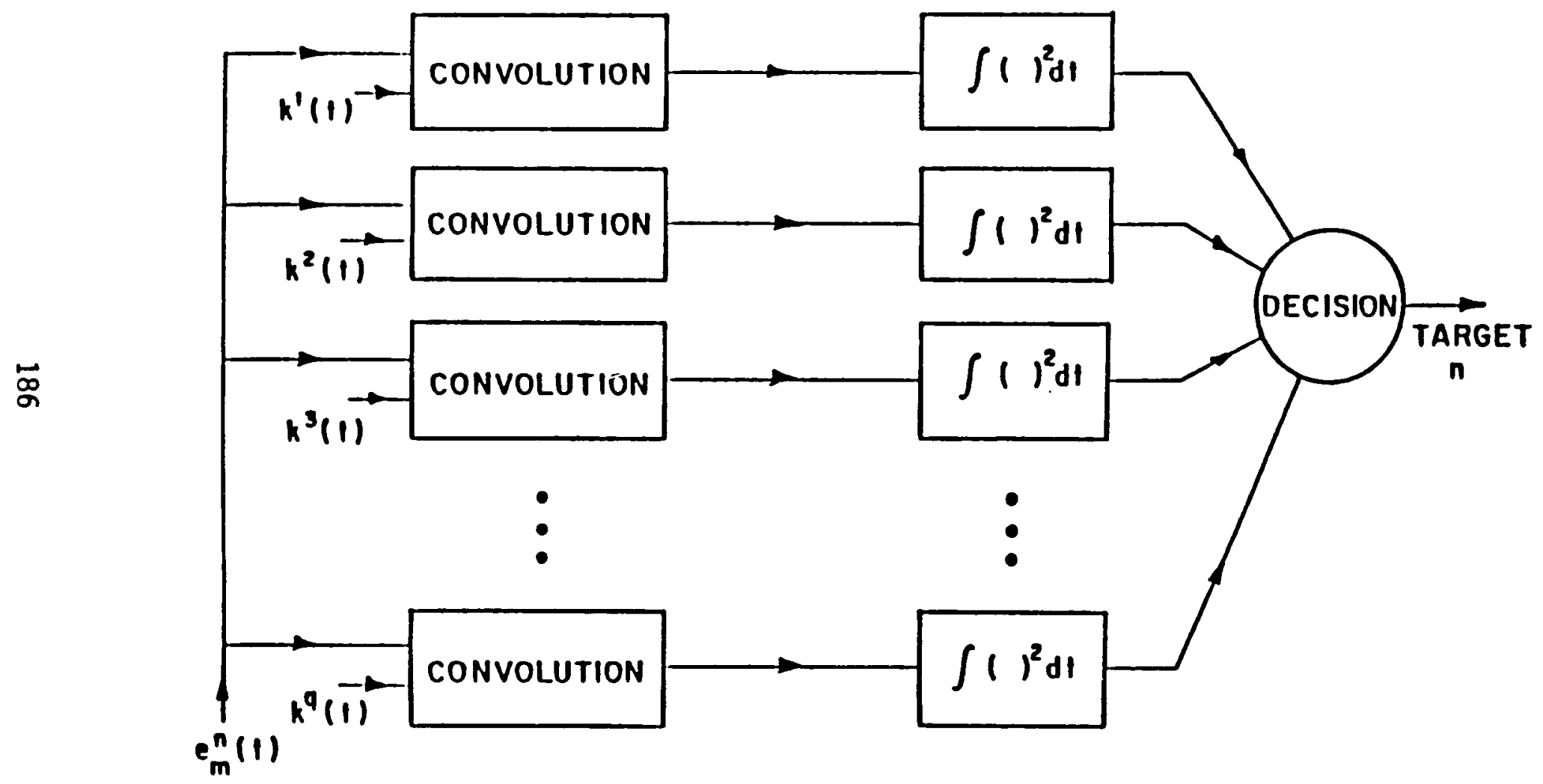

Figure B.3. Block diagram of a target discrimination scheme. 
energy content choices are discarded until only one choice remains. There are aiso ways of identifying targets using the natural resonances of the targets [32-35]. Using the poles derived from the K-pulse, these target identification methods can also be applied.

A word is in order concerning the effects of noise on a full scale target discrimination system. Low $Q$ targets will present the most difficulty, as there is not much energy to compare with at the late time. Their broadband Fourier synthesized or measured impulse responses are of short duration. Fortunately, very few practical targets appear to be low Q [36]. K-pulses for practical targets will continue to be obtained from the 0.S.U. compact range facility which supplies clutter-limited rather than noise-limited data. The proper place for assessment of notse effects is in the identification studies rather than K-pulse generation studies. All in all this appendix provides a feasible target discrimination scheme. 


\section{APPENDIX C \\ K-PULSE AND E-PULSE}

Recently, a concept called "Extinction-Pulse" (E-pulse) [11], which under certain conditions is closely associated with the K-pulse concept [2] was discussed in the ifterature. In this appendix, this relationship is discussed. The defintition for the $K$-pulse is given in Chapter II. An E-pulse is defined as a transient, finite duration waveform which annihilates the contribution of a select number of the natural resonances in the late time response [11]. Comparing the two definitions, if 1) the E-pulse is chosen such that all the natural resonances are annihilated, 2) the E-pulse contains no zero other than those coinciding with the poles of the targets, 3) the duration of the E-pulse is minimal, then it is the same as the K-pulse originally defined by Kennaugh. The natural E-pulse model in [11] is,

$$
e^{e}(t)=\sum_{m=1}^{2 N} c m_{m} g_{m}(t)
$$

where the $g_{m}(t)$ 's are the basis functions, the $c_{m}$ 's are real constants to be determined and $2 \mathrm{~N}$ is the number of poles to be cancelled. The E-pulse $\left[e^{e}(t)\right]$ has a duration of $T_{e}$. For a subsectional pulse basis function of duration $\gamma$,

$$
g_{m}(t)=u[t-(m-1) \gamma]-u(t-m \gamma) \text {, }
$$


where $u(t)$ is the unft step function. The duration of the E-pulse $\left(T_{e}\right)$ is chosen as $2 N n \pi / \omega_{l}[11]$, where $n$ is an integer and $\omega_{l}$ is the imaginary part of the $e^{\text {th }}$ pole-patr to be cancelled. Equation (C.1) can be written in the s-domain as

$$
E^{e}(s)=\left(\frac{1-e^{-s \gamma}}{s}\right) \sum_{m=1}^{2 N} c_{m} e^{-s(m-1) \gamma}
$$

where $\gamma=n \pi / \omega_{\ell}$. Since $N$ complex pole-pairs are to be cancelled by Equation (C.3), this function must also contain the corresponding complex zero-pairs.

The above expression is actually simflar to the "Method of Zero Insertion in Transform Domain" discussed by Gerst and Diamond [4] for lumped paramter system. One example discussed by them is

$$
K(s)=\left(\frac{1-e^{-\varepsilon s}}{s}\right) \prod_{i=1}^{2 N}\left[1-e^{-\varepsilon\left(s-s_{i}\right)}\right]
$$

where $s_{i}$ 's are the poles to be cancelled, $\varepsilon$ is an arbitrary number, and $2 \mathrm{~N}$ is the number of poles to be cancelled. This input waveform was also used by Kim et al. [10] as a K-pulse model for the circular loop with the duration of the K-pulse, $T=(2 N+1) \varepsilon$. As pointed out by Gerst and Diamond [4], the $\left(1-e^{-\varepsilon s}\right) / s$ factor, which is a pulse basis function, in Equation (C.4) can be replaced by any Laplace tranform function $G(s)$ which has a finite time duration. In other words, any other basis function can replace the pulse basis function in (C.4). Since $\varepsilon$ is arbitrary, it can be forced to cancel one of the poles in the $e^{\text {th }}$ complex pole-pair. That is, 


$$
1-e^{-\varepsilon\left(s_{2} s_{\ell}\right)}=0 \quad \text { and } \quad 1-e^{-\varepsilon\left(s_{2} s_{\ell}\right)}=0 \text {, }
$$

where $s_{\ell}^{*}$ is the complex conjugate of $s_{\ell}$. Solving for $\varepsilon$, gives $\varepsilon=n \pi / \omega_{\ell}$, which is the same as $r$ in Equation (C.3). Therefore, Equation (C.4) can be reduced by one term,

$$
K(s)=\left(\frac{1-e^{-E s}}{s}\right) \prod_{i=1}^{2 N-1}\left(1-e^{-E(s-s i)}\right) \text {, }
$$

and the duration of $K$-pulse is now $2 N \varepsilon$. Substituting $z=e^{-\varepsilon S}$, the above equation can be written as,

$$
K(s)=\frac{(1-z)}{s}\left[1+\sum_{m=1}^{2 N-1} a_{m} z^{m}\right]
$$

where the $q_{m}$ 's are functions of the poles. Performing the same operation to Equation (C.3),

$$
\left.E^{e}(s)=c_{1} \frac{(1-z}{s}\right)\left[1+\sum_{m=1}^{2 N-1}\left(\frac{c_{m+1}}{c_{1}}\right)_{2}^{m}\right] \text {. }
$$

Since $K(s)$ in Equation $(C .7)$ is the same order of polynominal of $z$ as $E^{e}(s)$ in Equation (C.8) and they both contain the same $2 N$ zeros in order to cancel the natural resonances, they are related by a real constant. Furthermore,

$$
\alpha_{i}=\frac{c_{j+1}}{c_{1}}
$$

The expression for $K(s)$ in Equation (C.6) shows explicitly how the poles are cancelled, which was concealed in Equation (C.1) and all the theory behind it [11]. Henceforth, all reference to the E-pulse model or the K-pulse model by Kim et al. will be referred to Equation (C.6). This concludes the disscussion that both the $K$-pulse model employed by 
Kim et al. [10] and the E-pulse model [11] come from the same expression used by Gerst and Diamond [4]. However, there is a strong distinction between the two applications. Kim et al. take the limit of the number of subsectional pulse basis functions to a large number (60); whereas, Rothwell et al. only use a small number (less than 20) of subsectional basis functions.

Next, the K-pulse model from this dissertation [Equation (2.22)] and the other K-pulse model [Equation (C.6)] are compared. The expression (C.6) has roots other than what was specified $\left(s_{i}\right)$. Namely,

$$
s_{\text {in }}=s_{i}+\frac{j 2 n \pi}{\varepsilon}
$$

are also zeros of Equation (C.6) for all integer $n$. Equation (2.22) which is the Laplace transform of Equation (2.17) does not have such a problem. To use Equation (C.6); the poles of the target must be known a priori. The approach presented in this dissertation does not have such a restriction. The only restriction this method may have is the bandlimited Fourier synthesized impulse responses used which limits the usable bandwidth of the K-pulse obtained.

The inverse Laplace transform of Equation (2.22) consists of an impulse at $t=0$ and a continuous function spanning over the $K$-pulse duration [Equation (2.17)]. The inverse Laplace tranform of Equation (C.6) is a set of rectangular pulses. Could they be related? There are two pieces of evidence that suggest they may be related. If Equation (C.6) is divided by $\varepsilon$ and $\varepsilon$ is taken to the limit of zero as $N$ is increased to infinity, the inverse Laplace transform of Equation (C.6) 
is an infinite set of impulses over the span of the K-pulse. This is because [16]

$$
\left.\lim _{\varepsilon \rightarrow 0} \frac{[u(t)-u(t-\varepsilon)}{\varepsilon}\right]=\delta(t),
$$

where $u(t)$ is the unit step function. On the other hand, the continuous portion of the K-pulse in Equation (2.17) can also be viewed as an infinite set of impulses over the duration of the K-pulse. Hence they are both infinite set of impulses.

The other evidence is the interpretion of Equation (C.10), as $E$ approaches zero, the extra zeros will go to infinity, leaving only the proper zeros in the finite plane. Hence, if the zeros in the finite plane of both models described in Equation (2.22) and Equation (C.6) correspond to all the poles of the target and none other, then the models must be related. In summary, this appendix has shown that the E-pulse model used by Rothwell et al. [11] and the K-pulse model used by Kim et al. [10] orignated from the same equation used by Gerst and Diamond [4] as an example. There are also very strong evidences that their model is also similar to the $K$-pulse model employed in this dissertation. 


\section{APPENDIX D}

\section{PROOF THAT A MEMORPHIC FUNCTION CAN BE FACTORED INTO}

\section{A RATIO OF TWO ENTIRE FUNCTIONS}

The following proof is reproduced from the notes by Prof. J. T. Scheik of the Mathematical Department at The Ohio State University: Let $r(z)$ be a memorphic function in the complex plane. The poles of $r(z)$ are listed as $z_{i} ; i=1, \ldots, \infty$. The higher order poles are also included in the list. Next, an entire function $f(z)$ is defined such that its zeros and their respective orders are the same as the poles of $r(z) . f(z)$ can be written [37] as,

$$
\begin{aligned}
& f(z)=z^{m} e^{h(z)} \prod_{i=1}^{\infty}\left(1-\frac{z}{z_{i}}\right) e^{p_{j}(z)} ; \\
& \text { where } p_{i}(z)=\sum_{k=1}^{i} \frac{1}{k}\left(\frac{z}{z_{j}}\right)^{k} ;
\end{aligned}
$$

$m$ is the order of the pole of $r(z)$ at $z=0 ; h(z)$ is an entire function, then

$$
f(z) r(z)=g(z) .
$$

$g(z)$ is an entire function, as all the poles of $r(z)$ are cancelled by $f(z)$, and $g(z)$ is now analytic everywhere. For $z \neq z_{i}$,

$$
r(z)=\frac{g(z)}{f(z)}
$$

This concludes the proof that a memorphic function $r(z)$ can be written as a quotient of two entire functions $g(z)$ and $f(z)$.

* Simflar proof is probably given in some textbooks. It is included here for the readers whose interests are in the mathematical properties of the K-pulse. 


\section{REFERENCES}

[1] Special Issue on Inverse. Methods in Electromagnetics, IEEE Trans. Antennas and Propagation, vol. AP-29, no. 2, March 1981.

[2] E. M. Kennaugh, "The K-pulse Concept", IEEE Trans. Antennas and Propagation, vol. AP-29, no. 2, Pp. 327-331, March 1981.

[3] L. Marin, "Natural-Mode Representation of Transient Scattered Fields", IEEE Trans. Antennas and Propagation, vol. AP-21, no. 6, pp. 808-819, November 1973.

[4] I. Gerst and J. Diamond, "The Elimination of Intersymbol Interference by Input Signal Shaping", Proc. IRE, vol. 19, no. 7. pp. 1195-1203, 1961.

[5] E. M. Kennaugh and J. H. Richmond, "Generalized Aperiodic Excitation in Transient Field Problems", presented at USNC-URSI Spring Meeting, Washington D. C., April 1972.

[6] J. B. Campbell, "Design of Input Waveform to Yield Time-limited Orthogonal Outputs", Tech. Report 3, Proj. 4610, Northeastern University, Boston, Mass., March 1963.

[7] J. B. Campbell, S. H. Chang, D. W. Fermental and N. T. Tsao-Wu, "Pulse Shaping by Manipulating Transform Zeros", Scientific Repurt \$1, Proj. 4610, Northeastern University, Boston, Mass., Dec. 1964.

[8] E. M. Kennaugh, "Transient Analysis of Radiation and Scattering Properties of a Thin Linear Antenna", an unsolicited proposal dated November 1975.

[9] E. M. Kennaugh, D. L. Moffatt and N. Wang, "The K-pulse and Response Waveforms for Non-uniform Transmission Lines", IEEE Trans. Antennas and Propagation, vol. AP-34, no. 1, pp. 78-83, January 1986.

[10] H. T. Kim, N. Wang and D. L. Moffatt, "K-pulse for A Thin Circular Loop", IEEE Trans. Antennas and Propagation, vol. AP-33, no. 12, Pp. 1403-1407, December 1985.

[11] E. Rothwe11, D. P. Nyquist, K. M. Chen, B. Drachman, "Radar Target Discrimination using the Extinction-pulse Technique", IEEE Trans. Antennas and Propagation, vol. AP-33, no. 9, pp. 929-936, September 1985. 
[12] Kennaugh and Cosgriff, "The Use of Impulse Response in Electromagnetic Scattering Problems", 1958 IRE National Convention Record Part 1, pp. 72-77.

[13] Kennaugh and Moffatt, "Transient and Impulse Response Approximation", Proceedings of IEEE, vol. 53, no. 8, pp. 893-901, August 1965.

[14] "Joint Services Electronics Program - Sixth Annual Report", Technical Report 710816-15, Section E-5, The Ohio State University Electroscience Laboratory, Columbus, Ohio, December 1983.

[15] Athans, Dertouzos, Spann, Mason, Systems, Networks and Computation: Multivariable Methods, McGraw-HITT, New York, New York, 1974.

[16] A. Papoults, The Fourter Integral and Its Applications, McGraw-Hill, New York, New York, 1962.

[17] A. Ralston, A First Course in Numerical Analysis, McGraw-Hili, New York, New York, 1965.

[18] F. J. Harris, "On the Use of Window for Harmonic Analysis with the Discrete Fourier Transform", Proceedings of IEEE, vol. 66, no. 1, pp. 51-83, Jan. 1978.

[19] Unpublished noces from E. M. Kennaugh.

[20] J. H. Richmond, "Computer Program for Thin-wire Structures in a Homogenous Conducting Medium". Technical Report \# 2902-12, The Ohio State University Electroscience Laboratory, Columbus, Ohio, August 1973.

[21] L. B. Felsen, "Comments on Early TIme SEM", IEEE Trans. Antennas and Propagation, vol. AP-33, no. 1, Pp. 118-120, January 1985.

[22] Chen and Westmoreland, "Impulse Response of a Conducting Sphere Based on Singularity Expansion Method", Proceedings of IEEE, vol. 69, no. 6, pp. 747-750, June 1981.

[23] Pathak and Kouyoumjian, "An Analysis of the Radiation from Apertures in a Curved Surface by GTD," Proceedings of IEEE, vol. 62, no. 11, pp. 1438-1447, November 1974.

[24] Mithouard and Hodge, "Electromagnetic Scattering by a Metallic Disk". Technical Report $710816-3$, The Ohio State University Electroscience Laboratory, Columbus, Ohio, September 1979. 
[25] T. Lee, "Approximate Methods for Obtaining the Complex Naturai Electromagnetic Oscillations of an Object", Ph. D. Dissertation, The Ohlo State University, Electrical Engineering Department, February 1984.

[26] Walton and Young, "The Ohio State Univeristy Compact Radar Cross-section Measurement Range", IEEE Trans. of Ant. and Prop., vol. AP-32, no. 11, pp. 1218-1223, November 1984.

[27] C. W. Chuang, "Scattering by a Rotationally Symmetric Conducting Body", Technical Report 714614-2, The Ohio State University Electroscience Laboratory, Columbus, Ohio August 1983.

[28] Moffatt, Lai and Lee, "Time-domain Electromagnetic Scattering by Open Ended Circular Waveguide and Related Structure", Wave Motion 6, pp. 363-387, June 1984.

[29] C. C. Huang, "Ray Analysis of EM Backscatter from a Cavity Configuration", Ph. D. dissertation, The Ohio State Univeristy, Electrical Engineering Department, 1982.

[30] D. L. Moffatt, "The K-pulse Waveform", "Measurement, Processing and Analysis of Radar Target Signatures" Workshop notes, vol. 2, The Ohio State University Electroscience Laboratory, Columbus, Ohio, Sept. 10-13, 1985.

[31] Rothwell, Chen, Nyquist, Gharsallah, Drachman, "Frequency Domain E-pulse Synthesis and Target Discrimination", Oral paper presented at IEEE APS - International Symposium, Vancouver, June 17-21, 1985.

[32] Mains and Moffatt, "Complex Natural Resonances of an Object in Detection and Discrimination", Technical Report \#3424-1, The Ohio State University Electroscience Laboratory, Columbus, Ohio, June 1974.

[33] Brittingham, Miller and Willows, "Pole Extraction from Realfrequency Information", Proceedings of IEEE, vol. 68, no. 2, pp. 263-273, February 1980.

[34] Moffatt, Young, Walt on and Leeper, "Resonant Structure NCTR", Final Report \#114190-2, The Ohio State University Electroscience Laboratory, Columbus, Ohio, January 1983.

[35] E. P. Bohley, "A Method for Discrimination of Radar Targets using Complex Natural Resonances". M. Sc. Thesis, The Ohio State University. Electrical Engineering Department, Dec. 1985. 
[36] C. Y. A. La1, "Improved Models for the Extraction and Application of Complex Natural Resonances to Target Identification". Ph. D. Dissertation, The Ohio State Untversity, Electrical Engineering Dept., Columbus, Ohto, March, 1986.

[37] R. M. Young, An Introduction to Nonharmontc Fourter Series, Academic Press, New York, New York, 1980. 